ORP-55237

Revision 0

\title{
Baseline LAW Glass Formulation Testing
}

Prepared for the U.S. Department of Energy

Assistant Secretary for Environmental Management

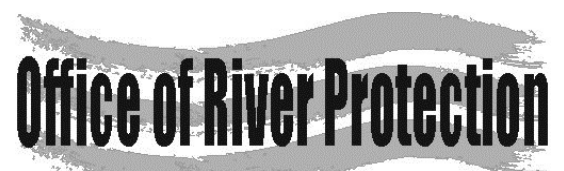

P.O. Box 450

Richland, Washington 99352 


\section{Baseline LAW Glass Formulation Testing}

\author{
A. A. Kruger \\ Department of Energy - Office of River Protection \\ C. Mooers \\ The Catholic University of America \\ G. Bazemore \\ The Catholic University of America \\ I. L. Pegg \\ The Catholic University of America \\ K. Hight \\ The Catholic University of America \\ S. T. Lai \\ The Catholic University of America
}
A. Buechele
The Catholic University of America
E. Rielley
The Catholic University of America
H. Gao
The Catholic University of America
I. S. Muller
The Catholic University of America
R. Cecil
The Catholic University of America

Date Published

April 2003

Prepared for the U.S. Department of Energy

Assistant Secretary for Environmental Management

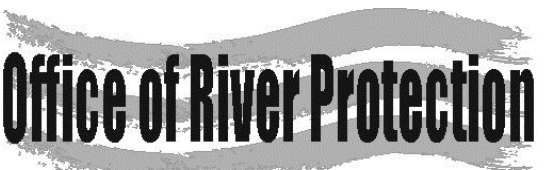

\section{P.O. Box 450}

Richland, Washington 99352 
ORP-55237

Revision 0

TRADEMARK DISCLAIMER

Reference herein to any specific commercial product, process, or service by tradename, trademark, manufacturer, or otherwise, does not necessarily constitute or imply its endorsement, recommendation, or favoring by the United States Government or any agency thereof or its contractors or subcontractors.

This report has been reproduced from the best available copy.

Printed in the United States of America 


\title{
Final Report
}

\section{Baseline LAW Glass Formulation Testing}

\author{
prepared by
}

Isabelle S. Muller and Ian L. Pegg

contributors

Hao Gan, Andrew Buechele, Elizabeth Rielley, Gina Bazemore, Richard Cecil, Kenneth Hight, Cavin Mooers, and Shan-Tao Lai

Vitreous State Laboratory

The Catholic University of America

Washington, DC 20064

$$
\text { for }
$$

Duratek, Inc.

and

Bechtel National, Inc.

April 3, 2003

Rev. 0, 8/5/03 
Document Title: Final Report: Baseline LAW Glass Formulation Testing

Document Number and Revision: VSL-03R3460-1, Rev. 0

Issue Date: 9/23/03

Performing Organization: Vitreous State Laboratory, The Catholic University of America

Test Specification: 24590-LA Wi-TSP-RT-01-009, Rev. 0

Test Exceptions: None

Test Plan: VSL-01T5600-3, Rey. 1

R\&T Focus Area(s): IAW Waste Form Qualification

Test Scoping Statement(s): VSL-20, Baseline ILAW Glass Formulations

\section{Completeness of Testing:}

This report describes the results of work and testing specified by the above-listed Test Specification(s), Test Plan(s), and Text Exception(s). The work and any associated testing followed established quality assurance requirements and was conducted as authorized. The descriptions provided in this test report are an accurate account of both the conduct of the work and the data collected. Results required by the Test Plan are reported. Also reported are any unusual or anomalous occuirences that are different from the starting hypotheses. The test results and this report have been reviewed and verified.

I.L. Pegg:
VSL Program Director/Principalf Investigator

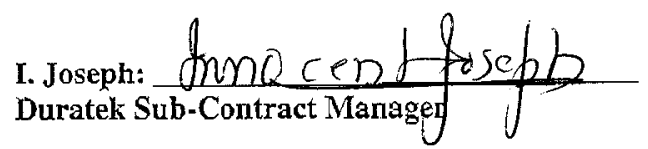

Date: $0 / 23 / 0]$

Date: $9 / 23 / 03$ 


\section{Table of Contents}

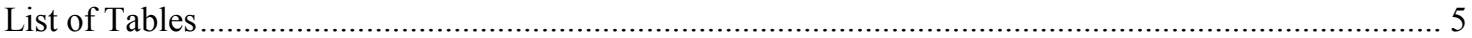

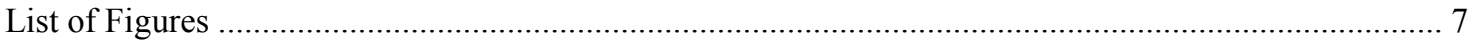

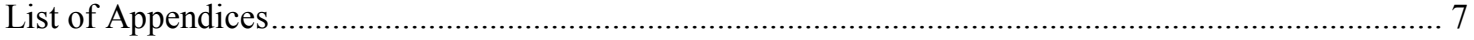

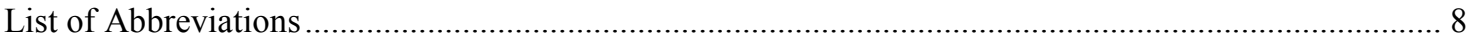

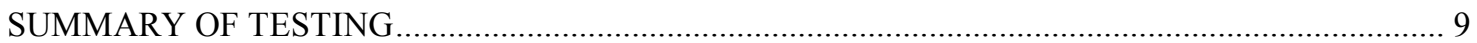

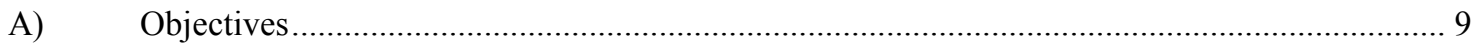

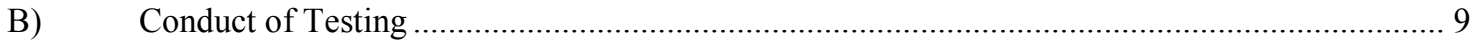

C) Results and Performance Against Objectives ...................................................................... 9

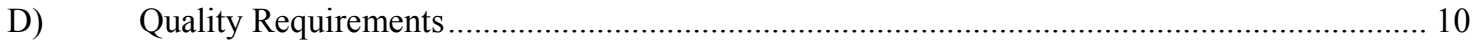

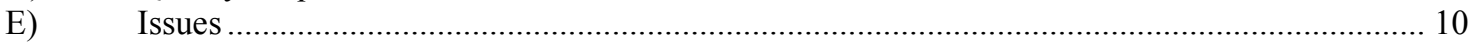

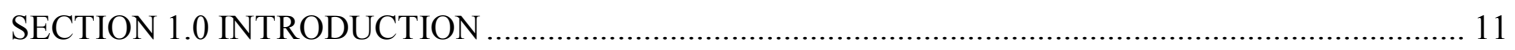

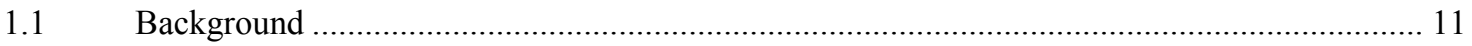

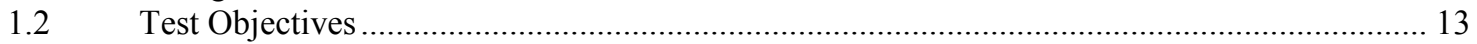

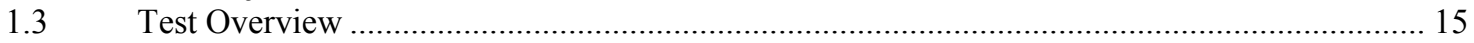

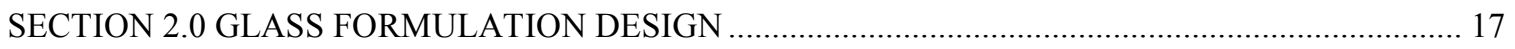

2.1 Glass Formulation Design Parameters and Constraints........................................................... 17

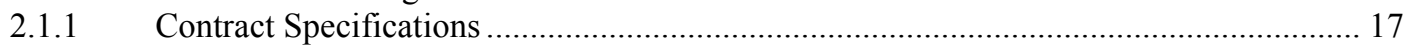

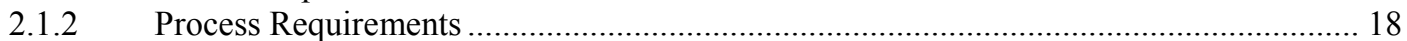

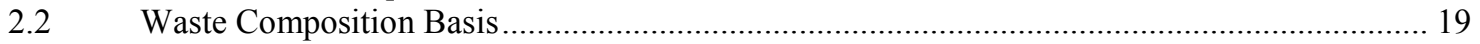

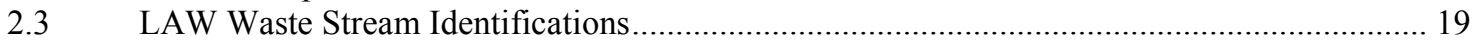

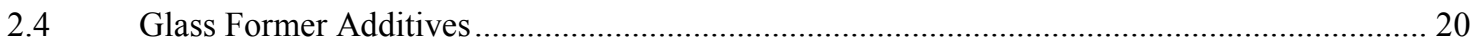

2.5 Glass Composition Development to Enhance Sulfate Incorporation.......................................... 20

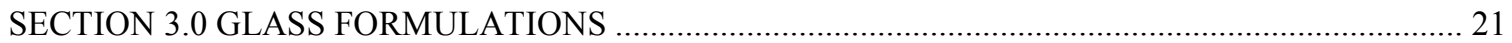

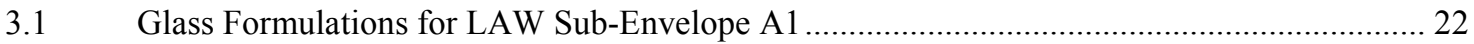

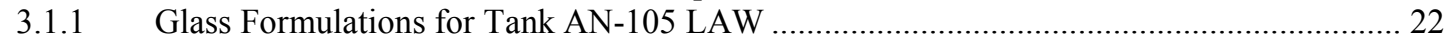

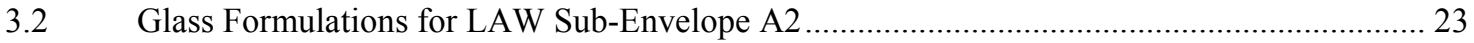

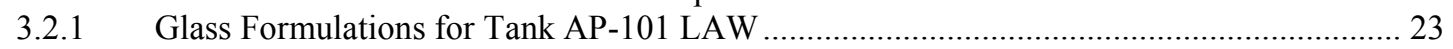

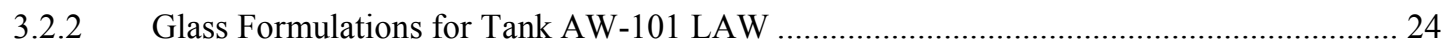

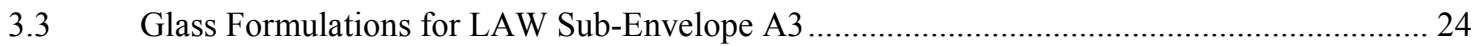

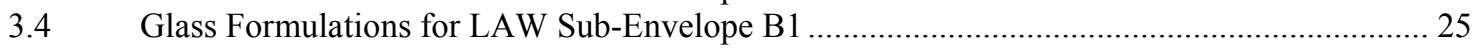

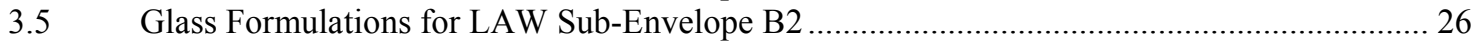

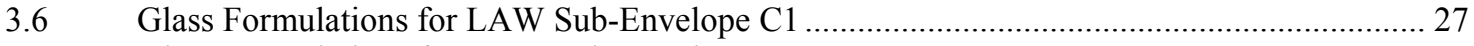

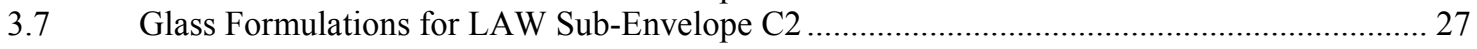

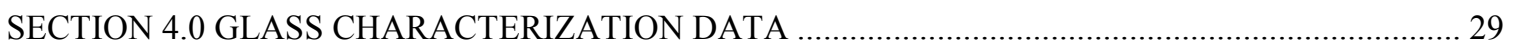

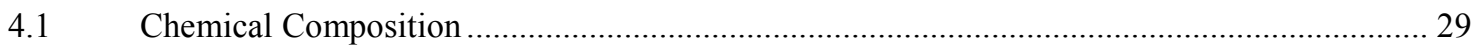

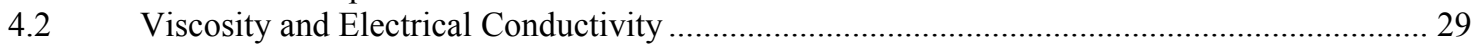

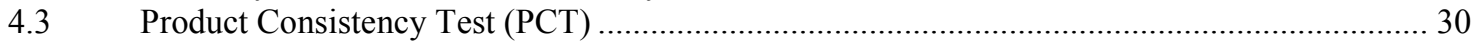

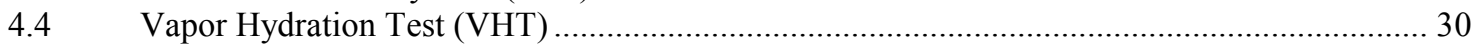

4.5 Liquidus Temperature and Crystallization Characteristics...................................................... 30

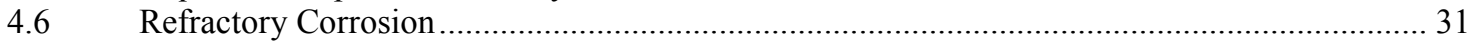

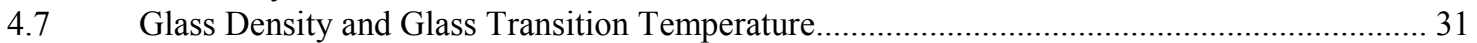

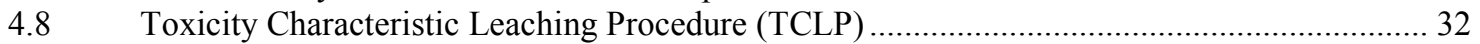

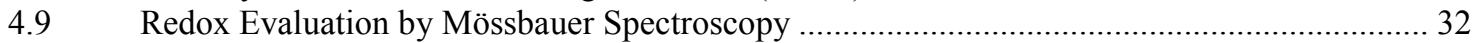

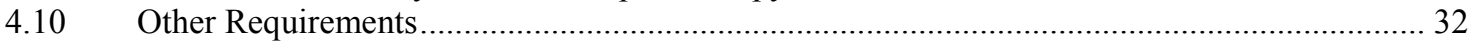

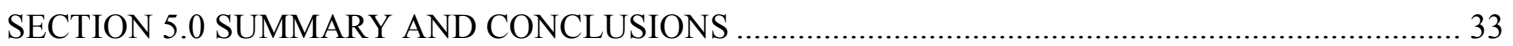




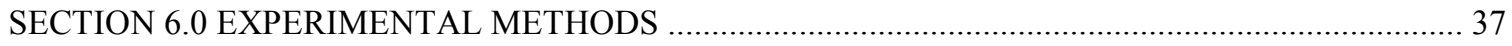

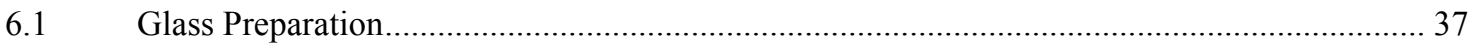

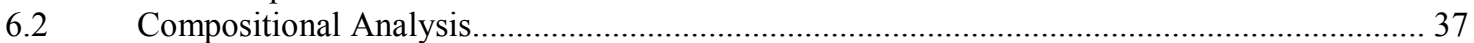

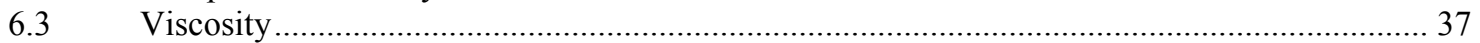

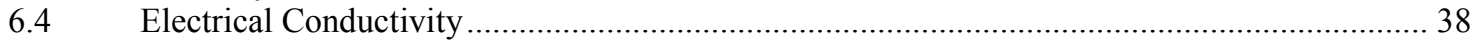

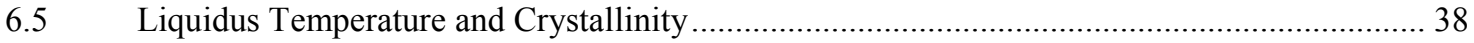

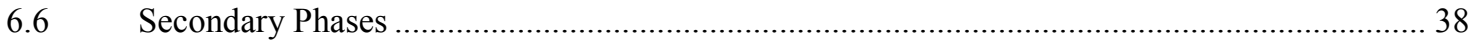

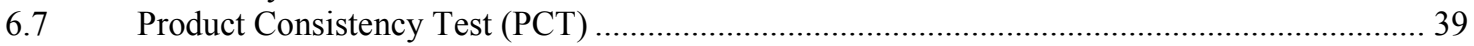

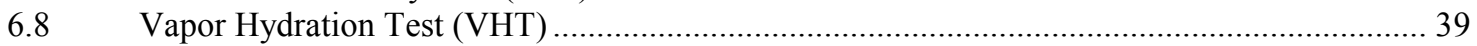

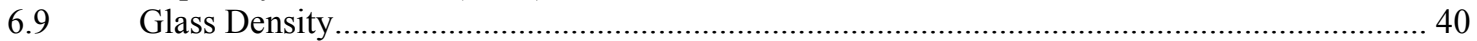

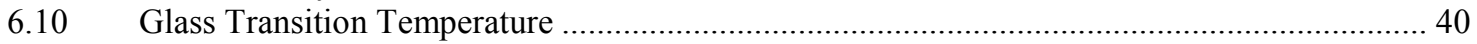

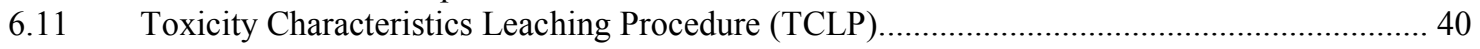

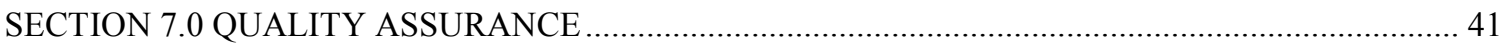

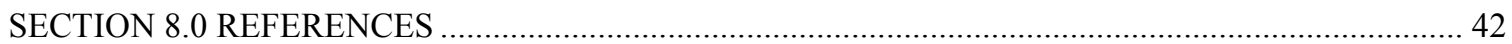




\section{List of Tables}

Table 1.1 Summary of ILAW Requirements.

$. \mathrm{T}-1$

Table 2.1 Compositions (Molarity) of the Nine LAW Tank Wastes as Given in TFCOUP Rev. 2.

Table 3.1 Waste Contribution to the Glass Composition for Tank AN-105 Supernatant (Batch 8) Per TFCOUP Rev. 2 and Target Compositions of High-Sulfate LAWA44 Glasses.

Table 3.2 Composition of Glasses for LAW Tank AN-105 Supernatant (Batch 8) per TFCOUP Rev. 2.

Table 3.3 Contribution to the Glass Composition for Tank AP-101 Supernatant Per TFCOUP Rev. 2.

Table 3.4 AP-101 Waste Composition as Reported in PNNL-13354 [8] and Waste Contribution to Glasses. ....................................................................................

Table 3.5 Composition of Glasses Tested for LAW AP-101 (per PNNL-13354 [8]).............T-9

Table 3.6 Compositions of LAW AW-101 Waste (Per TFCOUP Rev. 2) and the Glasses Tested. T-10

Table 3.7 Composition of LAW AN-104 (Batch 5) Per TFCOUP Rev. 2 and Composition of LAWA102 Glass. T-11

Table 3.8 Composition of LAW AZ-101 (Batch 2a) Per TFCOUP Rev. 2 and Waste Contribution to Glass.

Table 3.9 Composition of Glasses LAWB45 and LAWB60 for LAW AZ-101 Per TFCOUP Rev. 2 - High Waste Loading.

Table 3.10 Composition of Glasses Tested for LAW AZ-101 Per TFCOUP Rev. 2 High Waste Loading.

Table 3.11 Composition of Glasses Tested for LAW AZ-101 Per TFCOUP Rev. 2 Low Waste Loading.

Table 3.12 Composition of Glasses Tested for LAW AZ-101 Per TFCOUP Rev. 2 Alkali Variation. T-24

Table 3.13 Composition of LAW AZ-102 (Batch 2b) Per TFCOUP Rev. 2 and Waste Contribution to Glass.

Table 3.14 Composition of Glasses Tested for LAW AZ-102 Per TFCOUP Rev. 2..............T-28

Table 3.15 Composition of LAW AN-107 Per TFCOUP Rev. 2 and Composition of Glass LAWC22. $. \mathrm{T}-33$

Table 3.16 Composition of LAW AN-102 Per TFCOUP Rev. 2 and Waste Contribution to Glass T-34

Table 3.17 Composition of LAWC21 for AN-102 Composition Per TFCOUP Rev. 2..........T-35

Table 3.18 Composition of Glasses Tested for LAW AN-102 Per TFCOUP Rev. 2 ............. T-36

Table 4.1 XRF Analysis of LAWA44 Glasses for LAW AN-105. T-40

Table 4.2 XRF Analysis of Glasses for LAW AN-105 Per TFCOUP Rev. 2...................... T-41

Table 4.3 XRF Analysis of LAWA88 Glass for LAW AP-101 .................................... T-45

Table 4.4 XRF Analysis of Glasses for LAW AP-101 per PNNL-13354 [8] ..................... T-46

Table 4.5 XRF Analysis of Glasses for LAW AW-101 Per TFCOUP Rev. 2 ..................... T-47

Table 4.6 XRF Analysis of Glasses Tested for LAW AN-104......................................... T-48 


\section{List of Tables (continued)}

Table 4.7 XRF Analysis of Glasses Tested for LAW AZ-101.....................................T-51

Table 4.8 XRF Analysis of Glasses Tested for LAW AZ-102 ....................................... T-62

Table 4.9 XRF Analysis of Glasses Tested for LAW AN-107.......................................... T-68

Table 4.10 XRF Analysis of Glasses Tested for LAW AN-102 ........................................ T-69

Table 4.11 DCP Analysis of LAW Envelope A Glasses................................................... T-73

Table 4.12 DCP Analysis of LAW Envelope B Glasses............................................... T-79

Table 4.13 DCP Analysis of LAW Envelope C Glasses................................................ T-81

Table 4.14 Viscosity and Conductivity Data for LAW Envelope A Glasses........................T-82

Table 4.15 Viscosity and Conductivity Data for LAW Envelope B Glasses......................... T-83

Table 4.16 Viscosity and Conductivity Data for LAW Envelope C Glasses......................... T-87

Table 4.17 Results of the PCT at $90^{\circ} \mathrm{C}$ Per ASTM C1285-98 for LAW Envelope A

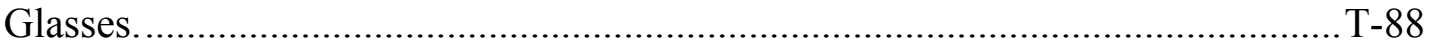

Table 4.18 Results of the PCT at $90^{\circ} \mathrm{C}$ Per ASTM C1285-98 for LAW Envelope B

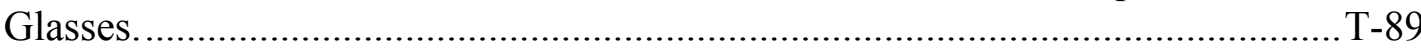

Table 4.19 Results of the PCT at $90^{\circ} \mathrm{C}$ Per ASTM C1285-98 for LAW Envelope C

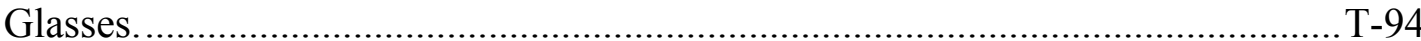

Table 4.20 Results of Vapor Hydration Test (VHT) at $200^{\circ} \mathrm{C}$......................................... T-95

Table 4.21 Results of Heat Treatment and SEM Analysis.................................................. T-97

Table 4.22 Results of K-3 Refractory Corrosion Testing............................................... T-100

Table 4.23 Glass Transition Temperature $\left(\mathrm{T}_{\mathrm{G}}\right)$ Measured by Differential Thermal Analysis for LAW Glasses............................................................................ T-102

Table 4.24 Glass Density......................................................................................... T-103

Table 4.25 Results (mg/l) from TCLP Testing (Performed at VSL; Analysis by DCP).

Table 5.1 Composition and Characterization Data for Glass LAWA44 for Tank AN-105 Supernatant (Batch 8) (Based on TFCOUP Rev.2).............................T-105

Table 5.2 Composition and Characterization Data for Glass LAWA88 for Tank AW-101 Supernatant (Batch 14) (Based on TFCOUP Rev.2)...........................T-106

Table 5.3 Composition and Characterization Data for Glass LAWA126 for Tank AP-101 Supernatant (Batch 1) (Based on TFCOUP Rev. 3) ..............................T-107

Table 5.4 Composition and Characterization Data for Glass LAWA102 for Tank AN-104 Supernatant (Batch 5) (Based on TFCOUP Rev.2)..............................T-108

Table 5.5 Composition and Characterization Data for Glass LAWB83 for Tank AZ101 Supernatant (Batch 2a) (Based on TFCOUP Rev.2). T-109

Table 5.6 Composition and Characterization Data for Glass LAWC22 for Tank AN-107 Supernatant (Batch 7) (Based on TFCOUP Rev.2).... T-110

Table 5.7 Composition and Characterization Data for Glass LAWC31 for Tank AN-102 Supernatant (Batch 3) (Based on TFCOUP Rev.2). 


\section{List of Figures}

Figure 1 Centerline canister cooling curve used for heat treatment of LAWA44CC and LAWA88CC in this work

Figure 2 Optical images of K-3 refractory coupons before corrosion testing ("unreacted") and after six-day corrosion testing in the indicated glass formulation [29] illustrating the "cracking" discussed in the text.

Figure 3 Mossbauer spectra for LAWA44 and LAWB83 showing all iron as Fe ${ }^{3+} \ldots \ldots \ldots \ldots \ldots . . . . . . .3$

Figure 4 Sulfate content measured in crucible melt glasses from this work and previous work $[1,2]$ as a function of their sodium content.

Figure 5 Plot of the sulfate to sodium molar ratio estimated for the LAW feeds for all 28 double-shell tanks, as reported in TFCOUP Rev. 3A [7] with the assumption that $50 \%$ of sodium and $75 \%$ of the sulfate in the solid fraction would be returned to the liquid feed. The data points are compared to the limits defining the LAW Envelopes.

Figure 6 Plot of the sulfate to sodium molar ratio estimated for the LAW feeds for all 149 of the single-shell tanks, as reported in TFCOUP Rev. 3A [7] with the assumption that $50 \%$ of sodium and $75 \%$ of the sulfate in the solid fraction would be returned to the liquid feed. The data points are compared to the limits defining the LAW Envelopes.

\section{List of Appendices}

Appendix 1 Estimate of the Sulfate to Sodium Ratio Expected in the LAW Feed Delivered for 28 Double Shell Tanks Per TFCOUP Rev. 3A.

Appendix 2 Estimate of the Sulfate to Sodium Ratio Expected in the LAW Feed Delivered for 149 Single Shell Tanks Per TFCOUP Rev. 3A. 


\section{List of Abbreviations}

\begin{tabular}{|c|c|}
\hline AES & Atomic Emission Spectroscopy \\
\hline ANL-LRM & Argonne National Laboratory Low-Activity Reference Material \\
\hline ANS & American Nuclear Society \\
\hline ANSI & American National Standard Institute, Inc. \\
\hline ASTM & American Society for Testing and Materials \\
\hline BNI & Bechtel National, Inc. \\
\hline DCP & Direct Current Plasma \\
\hline DCP-AES & Direct Current Plasma Atomic Emission Spectroscopy \\
\hline $\mathrm{DM}$ & DuraMelter ${ }^{\circledR}$ \\
\hline DOE & United States Department of Energy \\
\hline DTA & Differential Thermal Analysis \\
\hline EDS & Energy Dispersive X-Ray Spectroscopy \\
\hline HLW & High-Level Waste \\
\hline ILAW & Immobilized Low-Activity Waste \\
\hline LAW & Low-Activity Waste \\
\hline LDR & Land Disposal Restrictions (refer to 40 CFR 268) \\
\hline NIST & National Institute of Standards and Technology \\
\hline ORP & Office of River Protection \\
\hline PCT & Product Consistency Test \\
\hline PNNL & Pacific Northwest National Laboratory \\
\hline PNWD & Battelle, Pacific Northwest Division \\
\hline PSWP & Products and Secondary Wastes Plan \\
\hline QA & Quality Assurance \\
\hline QAPP & Quality Assurance Program Plan \\
\hline RPP-WTP & River Protection Project-Waste Treatment Plant \\
\hline SEM & Scanning Electron Microscopy \\
\hline $\mathrm{SEM} / \mathrm{EDS}$ & Scanning Electron Microscopy-Energy Dispersive X-Ray Spectroscopy \\
\hline SRTC & Savannah River Technology Center \\
\hline $\mathrm{T}_{\mathrm{g}}$ & Glass Transition Temperature \\
\hline TCLP & Toxicity Characteristic Leaching Procedure \\
\hline TFCOUP & Tank Farm Contractor Operation and Utilization Plan \\
\hline TTT & Time-Temperature-Transformation \\
\hline TWRS & Tank Waste Remediation System \\
\hline UTS & Universal Treatment Standard \\
\hline VHT & Vapor Hydration Test \\
\hline VSL & Vitreous State Laboratory \\
\hline WTP & Hanford Tank Waste Treatment and Immobilization Plant \\
\hline XRD & X-ray Diffraction \\
\hline XRF & X-ray Fluorescence Spectrometry \\
\hline
\end{tabular}




\section{SUMMARY OF TESTING}

\section{A) Objectives}

The major objective of the baseline glass formulation work was to develop and select glass formulations that are compliant with contractual and processing requirements for each of the LAW waste streams. Other objectives of the work included preparation and characterization of glasses with respect to the properties of interest, optimization of sulfate loading in the glasses, evaluation of ability to achieve waste loading limits, testing to demonstrate compatibility of glass melts with melter materials of construction, development of glass formulations to support ILAW qualification activities, and identification of glass formulation issues with respect to contract specifications and processing requirements.

\section{B) Conduct of Testing}

LAW waste composition data in the basis documents specified by WTP were reviewed and glass formulations were developed for testing. Crucibles melts of the glasses (about $400 \mathrm{~g}$ ) were prepared by melting mixtures of reagent grade or higher purity chemicals in platinum-gold crucibles at $1200^{\circ} \mathrm{C}$ for 75 minutes. The melts were stirred and the molten glass was poured onto a graphite plate to cool; samples of the resulting glass were then analyzed.

The viscosity and electrical conductivity of the melts were measured in the range of $950-1250^{\circ} \mathrm{C}$. Glass samples were heat treated at $700^{\circ} \mathrm{C}$ and $850^{\circ} \mathrm{C}$ to determine the bounds on the liquidus temperature and to determine the effect of heat treatment on crystallization. Since LAW glasses are designed to have a liquidus temperature of $950^{\circ} \mathrm{C}$ or less and generally show little tendency to crystallize, heat treatments were done at lower temperatures of $700^{\circ} \mathrm{C}$ and $850^{\circ} \mathrm{C}$. Secondary phase were identified and quantified by optical microscopy, $\mathrm{x}$-ray diffraction, or scanning electron microscopy in conjunction with energy dispersive x-ray spectroscopy. The Product Consistency Test (PCT), Vapor Hydration Test (VHT), and Toxicity Characteristic Leaching Procedure (TCLP) were performed on selected glass samples. The density of the glass at room temperature and the glass transition temperature were determined by pycnometry and differential thermal analysis, respectively.

\section{C) Results and Performance Against Objectives}

Glass formulation development and testing were performed to address eight of the Phase 1 LAW tank wastes: AP-101, AZ-101, AZ-102, AN-102, AN-104, AN-105, AN-107, and AW-101. Contractually compliant glass formulations that also meet the processing requirements were identified for all waste streams except LAW AZ-102. For the LAW AZ-102 waste stream, a target glass formulation at a lower waste loading was developed, as directed by DOE-ORP through the WTP Project. 
LAW waste composition data were reviewed and glass formulations were developed for testing. Crucible melts of the glasses were prepared and characterized. Sample of selected glasses were characterized for properties such as PCT, TCLP, VHT, viscosity, electrical conductivity, refractory corrosion, sulfate solubility, density, liquidus temperature, crystallization, and glass transition temperature. Optimization of sulfate was investigated and target glass compositions for processing were selected for a number of the Phase I LAW waste streams. The baseline glass formulation work was terminated by the WTP Project before development and selection of glass formulations for all of the Phase I LAW waste streams were complete. The WTP will rely on glass formulation work for actual wastes and melter testing to provide target compositions for these waste streams. All of the selected glasses met the contractual as well as the processing requirements (see Tables 1.1 and 5.1-5.7).

\section{D) Quality Requirements}

This work was conducted under an NQA-1 (1989) and NQA-2a (1990) Part 2.7 based quality assurance program that is in place at the VSL. This program is supplemented by a Quality Assurance Project Plan for RPP-WTP work that is conducted at VSL. Test and procedure requirements by which the testing activities are planned and controlled are also defined in this plan. The program is supported by VSL standard operating procedures that were used for this work. This work was not subject to DOE/RW-0333P, Rev. 10. This work was not subject to the requirements of WTP QAPjP for environmental regulatory data.

\section{E) Issues}

Due to the very high sulfate concentration in the LAW AZ-102 waste stream, a contractually compliant glass formulation containing $5 \mathrm{wt} \%$ waste $\mathrm{Na}_{2} \mathrm{O}$ was not viable. Therefore, as directed by DOE-ORP (via the WTP), a formulation at a lower sodium waste loading was developed and selected. This change has subsequently been incorporated into the WTP Contract. Glasses with very high sulfate loading tend to cause excessive K-3 refractory cracking due to the presence of high concentrations of $\mathrm{Li}_{2} \mathrm{O}$ ( $\geq 4$ $\mathrm{wt} \%$ ) that is added to improve sulfate solubility. 


\section{SECTION 1.0 INTRODUCTION}

\subsection{Background}

This report presents results from the Low Activity Waste (LAW) glass formulation development and testing performed at the Vitreous State Laboratory of the Catholic University of America between October 2001 and August 2002 in support of the River Protection Project-Waste Treatment Plant (RPP-WTP). A wide range of fully compliant glass formulations has been identified on the basis of characterization data from a total of 156 glasses that were prepared in this work.

As an integral part of the overall WTP design, glass formulation is of fundamental importance to waste form qualification and vitrification development activities. Glass formulation development provides data that are essential to the design of the RPP-WTP vitrification facility in the near term and to safe and reliable operation of that facility in the longer term. The data also form the bases upon which contractually and technically acceptable immobilized low activity waste (ILAW) products are developed.

Acceptable glass formulations for vitrification of Hanford LAW waste streams must meet a variety of product quality, processability, and waste loading requirements. To this end, a number of formulations were investigated during Part A [1] and Part B1 [2] of the WTP development program. The Part A work was directed towards the assessment of the viability of the contractually specified waste envelope and product requirements and identification of the risks associated with complying with those specifications. As a result, a significant fraction of the Part A LAW glass formulations were designed to address "envelope maximums" and are less likely to be designated as target glass formulations for waste processing. Building on the Part A work, a wide range of fully compliant LAW glass formulations was identified during Part B1 [2] based on the characterization data from about 135 glasses that were prepared during that phase. In contrast to the Part A formulations, the majority of the Part B1 formulations were developed around specific tank waste or blends of tank wastes with total waste oxide loadings ranging from 6 to $31 \mathrm{wt} \%$.

With the evolution of design and flow-sheet assumptions and revisions in waste compositions and product requirements, the glass formulation effort had to be responsive to new information since schedule constraints forced many Project design and development activities to be performed in parallel, rather than in series. One such change that had a major impact on the LAW glass formulation work was the deletion of the sulfate removal step from pretreatment. Consequently, towards the end of Part B1, the entire LAW glass development work was redirected to develop glass formulations that can accommodate higher amounts of sulfur to support the "vitrification only" strategy for sulfate management; "Vitrification only" strategy refers to processing of all of the sulfur in the waste streams through the WTP by vitrification without any sulfate removal in pretreatment. Other factors that influenced LAW glass development work included the evolving requirements of the ILAW Performance Assessment, changes in the Product 
Consistency Test (PCT) release specification, and incorporation of newer tank waste analysis data.

The WTP glass formulation strategy is described in the ILAW Product Compliance Plan (24590-WTP-PL-RT-03-001)[44]. As described in the plan, a number of glass formulations will be tested to establish a range of ILAW glass compositions that are compliant with Contract specifications. The glass compositions that result in products that meet specifications form a domain identified as the Qualified Glass Composition Region (QGCR). Within this region, target glass compositions will be identified based on expected compositions of the pretreated LAW feeds. To account for variability in the waste composition and uncertainty due to sampling and analysis, an upper and a lower limit will be established for each chemical in the waste. The composition of melter feed must lie within the bounds thus established in order to accept the feed for the melter. A model to predict the properties of vitrified glass (e.g., leach resistance) will be developed relating the glass composition and property. LAW melter feed compositions will be input to the predictive model to ensure that the vitrified glass composition is within the QGCR before transferring the waste to the melter feed vessel.

The experimental program to implement this strategy includes the following tasks:

- Estimate the composition of each waste type to be processed in the WTP from the best available data on LAW waste feeds.

- Develop and test non-radioactive glass formulations appropriate for a range of possible pretreated LAW compositions.

- Develop a QGCR for ILAW glass compositions, derived from the above step and any other valid data, that meets the product specifications.

- Develop one or more target glass compositions to envelope the types of wastes to be processed in WTP within the QGCR.

- Develop the ILAW composition variation region around each target glass composition that may result from process and waste composition variations.

- Prepare radioactive ILAW glasses (crucible melts) using waste treatability samples.

- Confirm the results of the non-radioactive demonstrations through crucible melts with waste treatability samples.

- Report the results in the ILAW QD, along with justification for any significant variation between radioactive and non-radioactive tests.

Readers are referred to the ILAW Product Compliance Plan for details of these activities.

Building on this information base from Part A and Part B1, early during the Bechtel National, Inc. (BNI) contract period, the RPP-WTP Project divided glass formulation activities to support LAW waste form qualification into the following tasks.

- LAW glass formulation to support melter runs with simulants.

- LAW glass formulation to support actual waste testing. 
- LAW baseline glass formulations.

- Update LAW glass formulations to support flow-sheet changes.

- LAW glass property-composition modeling and LDR-delisting support.

- TTT diagrams for LAW glasses.

Additional tasks to support waste form qualification were planned for the LAW Pilot melter at Duratek. The work presented in this report addresses only the third of these areas, "LAW baseline glass formulations" as specified in the associated Test Plan [3]. In October 2002, the WTP glass formulation effort was reprioritized with the result that work on the "Baseline Formulation" task was redirected. The principal objective of this report is, therefore, to present the data that were collected under this task up to that point in time. As waste feed projections are updated and flowsheet models and assumptions mature, the separate task to update LAW glass formulations to support flowsheet changes will assess the need to update formulations or develop new formulations to reflect the changes.

Target glass formulations for eight of the tank wastes were successfully identified during Part B1 [2] based on the LAW feed delivery information available at that time. The selection of the tanks was made on the basis of a schedule of feed delivery to the RPP-WTP [4] that has now been changed $[5,6,7]$. The present work for all but one of the tank wastes is based on the waste composition data given in revision 2 of the Tank Farm Contractor Operations \& Utilization Plan (TFCOUP) [5], as directed by the WTP Project. The actual waste characterization report [8] was used as the basis for the waste composition data for LAW tank AP-101.

Glass development work for eight LAW tank wastes is presented in this report. They include AP-101, AZ-101 (liquid fraction), AZ-102 (liquid fraction), AN-102, AN-104, AN-105, AN-107, and AW-101. The formulations cover total waste oxide loadings ranging from 7.2 to $26.2 \mathrm{wt} \%$ and, given the importance of sulfate on waste loading, many have also been tested at multiple sulfate contents, leading to 156 crucible melts of 60 different compositions.

\subsection{Test Objectives}

The principal objectives of this work were [3]:

- $\quad$ Review of the latest projected LAW feed compositions. The review included information from the latest revisions of the TFCOUP [5, 6, 7], basis of design, WTP flow-sheet, and other characterization data, as directed by the RPP-WTP Project. Actual waste characterization data [8] were used for AP-101 waste.

- Review of the existing glass formulations to determine whether reformulation of the baseline glass is needed for the waste stream of interest. 
- Preparation and characterization of glasses with respect to the properties specified in the Test Specification [9] and with respect to the properties of importance for the selected vitrification process. A summary of these requirements is given in Table 1.1.

- Evaluation of the ability to achieve the waste loading limits required by the RPP-WTP contract for each of the waste streams.

- Investigation of glass formulations to optimize sulfate loading in the LAW melter feeds. Sulfate is the waste loading limiting component for a number of LAW waste streams.

- $\quad$ Testing of selected glass formulations to demonstrate their compatibility with melter materials of construction. Since excessive corrosion of the melter materials of construction by the glass melt can severely limit melter life, this testing is a necessary part of baseline glass development.

- Development and selection of target glass formulations for each of the LAW waste streams.

- Identification of glass formulation issues with respect to the contract specifications or the process requirements.

- Development of glass formulation data to support the ILAW Qualification Documents.

Baseline glass formulation development was conducted for waste feeds from the first eight LAW tanks to be processed in the WTP, which are as follows:

- AP-101

- $\quad \mathrm{AZ}-101$

- $\mathrm{AZ}-102$

- $\mathrm{AN}-102$

- $\mathrm{AN}-104$

- $\mathrm{AN}-107$

- $\mathrm{AN}-105$

- $\mathrm{AW}-101$

Glass formulation for LAW tank AP-104/SY-101 will be addressed later, using composition data from actual waste sample analysis. This work will be documented in a separate report. Additional glass formulation work was not done for LAW tank AN-103 because the formulation selected earlier during Part B1 was deemed adequate.

An essential outcome of the previous formulation work is that sulfate may be the waste loading limiting constituent for a number of waste streams. Consequently, SubEnvelopes have been defined, [2] within each of the LAW waste Envelopes, that 
represent groupings of tanks that can be treated using common glass formulations based primarily on the relative amounts of sulfur and sodium in those streams. It was previously demonstrated that the contract waste loading limits could be achieved for all of the LAW streams identified during Part B1 of the contract. However, according to the more recent feed delivery plan $[5,6,7]$ the two LAW Envelope B tanks, AZ-101 and AZ-102, will be delivered separately rather than blended. This change required reformulation of the target glass compositions for the LAW Envelope B tanks, particularly in view of the very high ratio of sulfate to sodium in AZ-102. Consequently, development of suitable glass formulations for LAW Envelope B tanks formed a substantial portion of this work.

Revision 3A of the TFCOUP [7], which updates the compositions and delivery sequence for LAW and High-Level Waste (HLW) feeds ${ }^{1}$, indicated that the ratio of potassium to sodium in the LAW tank AP-101 had been increased significantly from that given in Revision 2. The glass formulation for LAW AP-101 feed, which was based on composition data from the earlier revision of the TFCOUP [5], was affected by this change. During the development of a glass formulation for LAW AP-101, particular attention was given to the effect of potassium and total alkali content on the properties of the glass.

\section{$1.3 \quad$ Test Overview}

The development of viable glass formulations for each of the waste envelopes is an iterative process in that prospective glasses are formulated, prepared in small crucible melts, and characterized to obtain the required property-composition information. The results are then analyzed, and revised compositions are generated in order to optimize the properties of the glass to the imposed constraints. The formulations are therefore "actively designed" rather than "statistically designed". The former approach is better suited to quickly and efficiently identifying acceptable solutions in the face of evolving constraints, whereas the latter generally provides more even coverage of the composition space of interest once that region can be sensibly specified. The LAW glass formulation efforts described in this report focused on "actively designed" compositions. The "actively designed" compositions will be complemented by a "statistically designed" matrix of compositions to support waste form qualification and development of propertycomposition models under a parallel task that is in progress at VSL $[10,11]$.

LAW baseline glass formulation work presented in this report is based on information from the TFCOUP, Rev. 2 [5] for all waste streams except LAW AP-101. The Project directed VSL/Duratek to use the actual waste characterization report, [8] instead of the TFCOUP [5], as the basis for the composition of LAW from tank AP-101. Waste composition data for all other tanks show good consistency in both revisions of the TFCOUP [5, 7] and compare well with characterization data. Estimated sodium additions in pretreatment were accounted for by using augmentation factors of 20/19.5 for Envelope A, 7.9/7.5 for Envelope B, and 20/17 for Envelope C, as described in the Basis of Design $[12,13]$. For an Envelope A glass, this means that, of the $20 \mathrm{wt} \% \mathrm{Na}_{2} \mathrm{O}$

\footnotetext{
${ }^{1}$ Note: Revision 4 of the TFCOUP became available after this work was completed.
} 
concentration in the glass, $19.5 \mathrm{wt} \%$ is from the LAW waste stream and $0.5 \mathrm{wt} \%$ is due to sodium additions in pretreatment. Information to estimate the compositions and amounts of HLW sludge leachate and wash solutions was not available from the WTP Project and, therefore, none was included in the waste contribution to the glass in this work. The effect of sulfur recycle from the vitrification process, assuming $80 \%$ retention in the glass $[2,14,15]$, was applied to all of the LAW glass formulation development work reported here $^{2}$.

Characterization of the "actively designed" glasses was performed in two stages. First, data with respect to properties that would impact acceptability were collected systematically as formulations were developed and the glasses characterized. The characterization data were then used to revise the compositions in order to optimize the properties of the glass with respect to the imposed constraints. These properties included viscosity, electrical conductivity, liquidus temperature, PCT and Vapor Phase Hydration Test (VHT). The specified limits for these properties as well as the preferred ranges for viscosity and electrical conductivity are given in Table 1.1 .

Data regarding other specifications in Table 1.1 were collected only for target glass compositions, either because glasses in general perform much better than the specifications, or because these properties show little change with glass composition over the range of interest. Such is the case for glass transition temperature $\left(T_{g}\right)$ and glass density. Consequently, these properties were only determined for the target glass compositions selected for each waste stream. For LAW glasses, the TCLP also falls into this category as a result of the generally very low concentrations of hazardous elements in the LAW stream. The results from Toxicity Characteristic Leaching Procedure (TCLP) testing of LAW glasses to support regulatory requirements will be presented in a separate report in response to separate RPP-WTP Test Guidance [17]. Results from VSL work conducted in Part A showed that the ANSI 16.1 leachability index of representative glass formulations greatly exceed the contract requirement (by 9 to 12 orders of magnitude). Consequently, no further testing was planned or performed with respect to this requirement.

Compatibility of the glass-melt with the melter materials of construction, and particularly contact refractories, is another important criterion in the selection of glass compositions for waste processing. Glass compositions were developed with special attention to this criterion and making full use of previous information that has been developed in this area [18]. Confirmatory testing of the selected target glass compositions for each of the tank wastes was performed to determine their corrosion characteristics with respect to Monofrax K-3, which is the glass-melt contact refractory for the LAW melter. The corrosion characteristics of the glass compositions with respect to candidate metal alloys for bubbler and off-gas components, including Inconel 690, is addressed separately [19].

\footnotetext{
${ }^{2}$ It should be noted that that value has since been revised to reflect more detailed information from melter testing. The current values are 85\% for Envelopes A and C and 70\% for Envelope B [16].
} 


\section{SECTION 2.0 \\ GLASS FORMULATION DESIGN}

The LAW glass formulation work presented in this report complements those from two other similar tasks, namely LAW glass formulations for melter testing [20] and LAW glass formulations for actual waste testing at Savannah River Technology Center (SRTC) and Battelle-Pacific Northwest Division (PNWD) [21]. The glass formulation work in each of these tasks draws on the results from the other tasks as well as from many previously tested glasses. In order to avoid redundancy, with the exception of a few cases in which specific results from earlier testing are included for comparison, only data on glasses that have not previously been reported have been included in this document.

\subsection{Glass Formulation Design Parameters and Constraints}

The design parameters and constraints that guided the present glass formulation work were similar to those employed in Parts A and B1; they are summarized in this Section. It is convenient to continue the same division of parameters: "Contract Specifications" and "Process Requirements." Glasses were formulated with the general objective of meeting all of these requirements.

\subsubsection{Contract Specifications}

The RPP-WTP contract provides the LAW envelope composition specifications and the specifications that the ILAW product must meet. This leads to the following design parameters and associated constraints:

- The loading in the glass of $\mathrm{Na}_{2} \mathrm{O}$ from the waste must be greater than $14 \mathrm{wt} \%$ for LAW Envelope A, $5 \mathrm{wt} \%$ for LAW Envelope B, and $10 \mathrm{wt} \%$ for LAW Envelope C (Contract Specification 2.2.2.2).

- The normalized mass loss of sodium, boron, and silicon under PCT leaching over 7 days at $90^{\circ} \mathrm{C}$ per ASTM C 1285-98 (Contract Specification 2.2.2.17.2) must be less than $2.0 \mathrm{~g} / \mathrm{m}^{2}$; the PCT procedure is described in Section 6.7.

- $\quad$ The alteration rate on the VHT at $200^{\circ} \mathrm{C}$ for seven days must be less than $50 \mathrm{~g} / \mathrm{m}^{2} /$ day (Contract Specification 2.2.2.17.3). However, for consistency with previous data [2] and per the DOE-approved VHT procedure in the Products and Secondary Wastes Plan (PSWP) [22], the majority of the tests were performed using a more conservative 24-day test duration; the VHT procedure is described in Section 6.8.

- Glass transition temperature (Contract Specification 2.2.1.1); the test procedure is described in Section 6.10. 
- $\quad$ Time-Temperature-Transformation (TTT) diagram (Contract Specification 2.2.2.6.3); development of TTT diagrams will be addressed in a separate task, at a later date.

- $\quad$ TCLP leaching performance and biotoxicity testing (Contract Specification 2.2.2.20). The biotoxicity tests were not planned under this work.

- $\quad$ Compressive strength (Contract Specification 2.2.2.18); compressive strength measurements were not planned under this work.

- Compressive strength after thermal, radiation, biodegradation and immersion per ANSI/ANS 16.1; compressive strength measurements were not planned under this work.

\subsubsection{Process Requirements}

In addition to the above requirements, the treatment process itself imposes an additional set of constraints on glass formulation. In particular, there are constraints on the properties of the glass melt for it to be processable in the joule-heated DuraMelter vitrification system that is part of the treatment process. These constraints form part of the interface between the expected melter design, operating methodology, and the glass formulation effort. The constraints are therefore subject to revision as the Project evolves. The process requirements on glass formulations employed in this work are:

- $\quad$ Glass should satisfy liquidus temperature limit $\left(950^{\circ} \mathrm{C}\right.$; see below); the test procedure is described in Section 6.5.

- Glass melt should satisfy the viscosity limits (the preferred range is $40-80$ poise at $1150^{\circ} \mathrm{C}$ ); the test procedure is described in Section 6.3.

- Glass melt should satisfy the electrical conductivity limits (0.2-0.7 siemens/cm at $1100-1200{ }^{\circ} \mathrm{C}$ ); the test procedure is described in Section 6.4 .

For WTP glass formulation work, an operational, rather than a true thermodynamic definition of liquidus temperature is used because of the presence of secondary phases (e.g. noble metal phases) in a number of glasses even at high temperatures. The "operational liquidus" temperature is defined as the temperature above which heat treatment of the glass sample for 20 hours results in less than one volume percent of non-settling secondary phases. In contrast to the HLW glasses, the distinction is largely irrelevant for LAW glasses because they satisfy the $950^{\circ} \mathrm{C}$ liquidus temperature limit both in terms of the strict thermodynamic as well as the "operational liquidus" definitions. 
Glass melts invariably react to some extent with materials used in melter construction. The selected glass formulations must be compatible with melter materials in order to maximize melter lifetime and minimize down time due to maintenance and change-out. Glass formulation work in Part B1 was performed with feedback from parallel materials corrosion studies [18]. The current tests followed a similar approach and made use of knowledge and data from past and continuing corrosion studies such that melt corrosivity was considered a secondary process requirement. Confirmatory refractory corrosion tests were conducted on many glasses to ensure that the selected baseline formulations continue to compare favorably with respect to corrosivity to benchmark formulations from other facilities [18].

\subsection{Waste Composition Basis}

The primary source of waste composition data for this work was the TFCOUP, Revision 2 [5]. For LAW tank AP-101 waste, the actual waste characterization data [8], was used as the primary source of waste composition. This information for LAW AP-101 is reflected in later revisions of the TFCOUP [6, 7]. The compositional effects in pretreatment were limited to inclusion of the Project baseline sodium addition assumptions $[12,13]$ and recycle was limited to the net effect of an assumed $80 \%$ retention of sulfur in the melter $[14,15,22-25]$. At steady-state recycle, this gives rise to a $25 \%$ increase in the sulfur content in the feed; a stoichiometric amount of sodium is also added because off-gas testing during previous melter tests [14, 15, 22-25] have shown that the majority of sulfur emitted is present as alkali sulfate particulate and sodium is the predominant alkali in the glass.

\subsection{LAW Waste Stream Identifications}

Identification of the tank wastes studied and their classification into LAW SubEnvelopes, based on Part B1 glass formulation work, are given below.
LAW Sub-Envelope A1:
AN-105
LAW Sub-Envelope A2:
AP-101, AW-101
LAW Sub-Envelope A3:
AN-104
LAW Sub-Envelope B1:
AZ-101
LAW Sub-Envelope B2:
AZ-102
LAW Sub-Envelope C1:
AN-107
LAW Sub-Envelope C2:
AN-102

The compositions of the tank wastes, based on the TFCOUP Rev. 2, Case 3S6E [5] are given in Table 2.1.

It is recognized that the effects on the expected LAW melter feed compositions from blending of the LAW supernate with HLW supernate, leachate, waste solutions, and 
HLW and LAW recycle streams, etc., also needs to be considered but this information from the WTP flow-sheet model was not available for use in this work.

\subsection{Glass Former Additives}

Glass former additives are mixed with the waste stream to produce a melter feed that yields the desired target glass composition. The amounts of glass former additives depend on the composition of the waste stream and the composition of the product glass. The LAW glass former additives are kyanite, wollastonite, olivine, zircon, boric acid, lithium carbonate, iron oxide, zinc oxide, and rutile. Kyanite supplies $\mathrm{Al}_{2} \mathrm{O}_{3}$ and $\mathrm{SiO}_{2}$. Wollastonite contains $\mathrm{CaO}$ and $\mathrm{SiO}_{2}$. Olivine provides $\mathrm{MgO}$ and $\mathrm{SiO}_{2}$. Boric acid $\left(\mathrm{H}_{3} \mathrm{BO}_{3}\right)$ is the source for $\mathrm{B}_{2} \mathrm{O}_{3}$. Lithium carbonate $\left(\mathrm{Li}_{2} \mathrm{CO}_{3}\right)$ provides $\mathrm{Li}_{2} \mathrm{O}$. Rutile $\left(\mathrm{TiO}_{2}\right)$ is the mineral used as the source for $\mathrm{TiO}_{2}$. $\mathrm{ZnO}$ and $\mathrm{Fe}_{2} \mathrm{O}_{3}$ are used as the source for $\mathrm{Zn}$ and Fe oxides in the glass. To the extent possible, raw materials that are traditionally used in the glass industry are used as LAW glass former additives. Minerals, rather than high purity raw materials are used, where possible, to minimize raw material cost without impacting product glass quality. Use of oxide forms of the raw materials, as opposed to carbonates or hydroxides, reduces the amount of gases that are evolved during melting.

$\mathrm{B}_{2} \mathrm{O}_{3}$ and $\mathrm{SiO}_{2}$ are borosilicate glass formers. $\mathrm{Li}_{2} \mathrm{O}$ is added as a flux that increases sulfate solubility in glasses. The remaining components are added to the glass for various reasons such as improving chemical durability, reducing refractory corrosion, reducing metal corrosion, increasing sulfate solubility, etc.

\subsection{Glass Composition Development to Enhance Sulfate Incorporation}

During Part A and early during Part B1 a sulfate removal step was part of the pretreatment process because of issues associated with vitrification of high-sulfate waste streams. Towards the end of Part B1 the sulfate removal process was deleted due to its cost, complexity, and excessive amounts of secondary waste streams. Instead a "vitrification only" strategy was adopted for sulfate management. Due to the rather late adoption of this strategy and the limited time frame in which to demonstrate its viability at LAW Pilot Melter scale, the development of acceptable glass formulations was expedited. Accordingly, the schedule allowed relatively little effort to be directed towards optimizing a glass formulation once an acceptable one for LAW Pilot Melter testing was identified. Consequently, the present effort focused on refinement and optimization of those glass formulations, particularly with respect to sulfate loading and assessment of their robustness with respect to composition variations. The work included formulation and preparation of crucible melts and measurement of their sulfate solubilities and rates of refractory corrosion. 


\section{SECTION 3.0 GLASS FORMULATIONS}

The target glass formulations and their relationships to the corresponding waste stream compositions are discussed in this section. The characterization data obtained on these glasses are presented in Section 4. In view of the large amount of tabulated data, the organization of that information is summarized below. The composition data are grouped together according to the LAW Sub-Envelope to which they refer.

- LAW glass formulations investigated in this work along with the corresponding waste compositions are given in Tables 3.1 to 3.18 .

- Results of compositional analysis by $x$-ray fluorescence (XRF) of the glasses are given in Tables 4.1 to 4.10. An "Sx" suffix with $\mathrm{x}=1,2,3,4$ at the end of a glass composition designation refers to a glass made with excess sulfate. A "W" at the end of a sample designation indicates that the glass sample was ground and washed to remove phase separated and interstitial sulfate before composition analysis in order to more closely estimate the amount of sulfur dissolved in the glass matrix.

- Results of compositional analysis by Direct-Current Plasma Optical Emission Spectroscopy (DCP) on dissolved glasses are given in Tables 4.11 to 4.13. The DCP data provide information on lithium and boron, which are not determined by XRF, and complementary information on other elements.

- Results from viscosity and electrical conductivity measurements are presented in Tables $4.14-4.16$.

- Results from PCT measurements are presented in Tables 4.17-4.19.

- Results from VHT measurements are presented in Table 4.20.

- Results from heat treatment of the glasses to determine liquidus temperature bounds are presented in Table 4.21.

- Results from refractory corrosion measurements are presented in Table 4.22.

- Results from glass transition temperature measurements are presented in Table 4.23.

- Results from glass density measurements are presented in Table 4.24.

- The target glass compositions selected for each waste stream along with relevant properties are given in Tables 5.1-5.7. 


\subsection{Glass Formulations for LAW Sub-Envelope A1}

Of the three waste feeds (AN-105, AN-103, and AP-104/SY-101) listed in the Test Specification [9] for LAW Sub-Envelope A1, only AN-105 was tested in this work. A total of 14 crucible melts were prepared as part of the glass development and optimization for LAW Sub-Envelope A1 waste feeds for AN-105. Based on a review of the waste composition data for AN-103, it was determined that no supplemental work was needed at this time for LAW AN-103 waste beyond that reported earlier [2]. Work on LAW AP-104/SY-101 was deferred by the WTP Project until actual waste sample characterization data would become available.

\subsubsection{Glass Formulations for Tank AN-105 LAW}

The waste feed compositions for the first batch of tank AN-105, as described in TFCOUP Rev. 2 [5] and Rev. 3 [6,7], are close to that used during Part B1 glass formulation work [2]. The current target glass composition, LAWA44, was tested successfully on the DM100 melter [26] and the LAW Pilot Melter [27] during 2002.

The second batch of AN-105 waste feed is expected to contain almost double the amount of $\mathrm{SO}_{3}$ as compared to the first batch [7]. As a result, the $\mathrm{SO}_{3}$ concentration of the LAWA44 glass at $19.5 \mathrm{wt} \%$ waste $\mathrm{Na}_{2} \mathrm{O}$ loading would increase from $\approx 0.2 \mathrm{wt} \%$ to $\approx 0.4 \mathrm{wt} \%$ when the waste composition basis is changed from the first to the second batch. The higher sulfate variation of the LAWA44 glass composition was not investigated during Part B1 because only the lower sulfate content feed was cited for this tank at that time. Accordingly, the present work investigated the sulfate solubility limits and the potential for enhancement of the sulfate loading potential of LAW AN-105 glasses. The target compositions of the glasses that were tested are given in Tables 3.1 and 3.2. XRF analysis results are given in Tables 4.1 and 4.2 and results of DCP analyses are given in Table 4.11. Formulation LAWA44 was prepared at two sulfate levels of 1.48 and $2.5 \mathrm{wt} \% \mathrm{SO}_{3}$. In both cases, a sulfate layer formed on the melt surface. XRF analysis revealed the sodium to be about 10 to 15 relative $\mathrm{wt} \%$ low in the glass, resulting in part to its incorporation into the separated sodium sulfate layer. In an attempt to compensate for this sodium loss, formulation LAWA44SX was prepared with $1.5 \mathrm{wt} \%$ excess $\mathrm{Na}_{2} \mathrm{O}$, or a new target $\mathrm{Na}_{2} \mathrm{O}$ concentration of $21.5 \mathrm{wt} \%$ in the glass. However, XRF analysis showed that the glass made from this formulation was still low in sodium. The maximum sulfate concentration in all these crucible glasses remained around $0.5-0.6 \mathrm{wt} \%$. Since, in an actual dynamic melter environment, the onset of sulfate layer formation occurs at concentrations below the saturation level, the LAWA44 formulation may not be suitable for the second batch of LAW AN-105 waste with higher sulfate concentration.

Five new formulations (see Table 3.2) were prepared and tested to determine the effect of various glass forming oxide additives on sulfate solubility. These glass compositions were based on LAWA44 and were prepared at various target sulfate concentrations. The LAWA44 composition was one of the earliest formulations selected 
during Part B1 [2] and, as a result, has a somewhat higher iron content than subsequently selected formulations. Consequently, in two of the glasses, LAWA108 and LAWA109, all or half of the iron oxide in LAWA44 was replaced with equal amounts of silica and boron oxide, respectively. As we have observed previously [2], decreasing the $\mathrm{Fe}_{2} \mathrm{O}_{3}$ content to around $3.5-5 \mathrm{wt} \%$ produced a slight increase in sulfate solubility whereas further decrease to $0 \mathrm{wt} \% \mathrm{Fe}_{2} \mathrm{O}_{3}$ produced no further benefit. In glass composition, LAWA110, half of the iron oxide in LAWA44 was replaced with equal amounts of aluminum, boron, and calcium oxides. Glasses LAWA111 and LAWA133 were prepared by replacing all or half of the iron oxide by calcium oxide. After lithium, calcium oxide has been observed to have one of the strongest beneficial effects on sulfate solubility [2]. As a result, the greatest increase (about 40\%) in sulfate solubility was observed at the highest calcium level, which was achieved by replacing all of the iron oxide with calcium oxide.

This work clearly shows that there is room for enhanced sulfate solubility in SubEnvelope A1 glasses beyond what was achieved in the LAWA44 composition. Such enhancements would be needed to maintain high waste loadings for the higher-sulfate dissolved saltcake batches. However, the work was terminated by the WTP Project before its completion and consequently, no new target glass formulation was selected.

\subsection{Glass Formulations for LAW Sub-Envelope A2}

LAW waste feeds from tanks AP-101 and AW-101 are designated as LAW SubEnvelope A2. The previous target glass composition for this Sub-Envelope, LAWA88, was designed based on the composition of LAW AW-101 waste feed. The major waste contributions to this glass were $19.5 \mathrm{wt} \% \mathrm{Na}_{2} \mathrm{O}, 2.5 \mathrm{wt} \% \mathrm{~K}_{2} \mathrm{O}$, and $0.2 \mathrm{wt} \% \mathrm{SO}_{3}$. LAWA88 was designated as the target glass composition for AP-101 waste feed based on the composition given in TFCOUP Rev.2 [5]. However, more recent information based on characterization data and reflected in TFCOUP Rev. $3[6,7]$ indicate substantial changes to the waste composition that require additional glass formulation development, as discussed below. Fifteen crucible melts were prepared as part of the glass development and optimization for this LAW Sub-Envelope.

\subsubsection{Glass Formulations for Tank AP-101 LAW}

The compositions of AP-101 LAW feed based on TFCOUP Rev. 2 (Case 3S6E) and the PNWD actual waste characterization report are given in Tables 3.3 and 3.4, respectively. The actual waste characterization data for LAW AP-101 has been incorporated into Rev. 3 of the TFCOUP [6]. As can be seen from these tables, the $\mathrm{K}_{2} \mathrm{O}$ concentration increases from $8.83 \mathrm{wt} \%$ to $15.72 \mathrm{wt} \%$ and the $\mathrm{SO}_{3}$ concentration increases from $1.17 \mathrm{wt} \%$ to $1.41 \mathrm{wt} \%$ as the waste composition basis is changed from TFCOUP Rev. 2 to Rev. 3. Due to this composition change, further glass development work was required to determine the effect of the higher alkali $\left(\mathrm{Na}_{2} \mathrm{O}+\mathrm{K}_{2} \mathrm{O}\right)$ content on both sulfate loading, glass durability, and refractory corrosion. Target compositions of the glasses that were studied are given in Tables 3.3, 3.4, and 3.5. Results of composition analysis by 
XRF are given in Tables 4.3 and 4.4. Results of DCP analyses are given in Table 4.11. The initial glass formulation development work was based on the results of earlier work $[2,28]$, keeping the total alkali $\left(\mathrm{Na}_{2} \mathrm{O}+\mathrm{K}_{2} \mathrm{O}\right)$ content at $22 \mathrm{wt} \%$, similar to that in LAWA88. Additional glasses were prepared and characterized at lower waste loadings. An earlier set of glass formulations, previously reported [28] as part of the glass formulation work in support of an actual waste sample vitrification at PNWD led to the selection of glass LAWA126 at $18.46 \mathrm{wt} \% \mathrm{Na}_{2} \mathrm{O}$ and $3.88 \mathrm{wt} \% \mathrm{~K}_{2} \mathrm{O}$ as a target composition for LAW Sub-Envelope A2. K-3 refractory corrosion was a major factor in the decision not to increase the total alkali content of the target glass composition.

A set of crucible melts was prepared to investigate the effect of iron, zinc, boron, and lower waste loading on the properties of glasses for LAW AP-101. These composition variations indicated no significant improvements in sulfate solubility or other glass properties and, therefore, LAWA126 was retained as the target glass composition for the vitrification of LAW AP-101 waste.

\subsubsection{Glass Formulations for Tank AW-101 LAW}

Glass formulations prepared and characterized for LAW AW-101 waste are given in Table 3.6. The results of composition analysis by XRF are given in Table 4.5 and the results of DCP analyses are given in Table 4.11. The development work was limited to investigating the effect of significantly higher calcium (achieved at the expense of iron) on sulfate solubility and other glass properties. The new glass formulation, LAWA112, was tested at an excess sulfate content of 1.5 and $2.5 \mathrm{wt} \%$ in, respectively, LAWA112S1 and LAWA112S2. In both cases, the sulfate solubility was improved to around $1.1 \mathrm{wt} \%$ $\mathrm{SO}_{3}$ as compared to about $0.7 \mathrm{wt} \% \mathrm{SO}_{3}$ for LAWA88. However, the measured viscosity was one of the lowest for LAWA glasses and K-3 refractory corrosion rate was high. LAWA88 was, therefore, maintained as the target glass composition for LAW AW-101 waste. As can be seen from Table 3.6, the same target glass composition, LAWA88, can be used for tank AW-101 LAW batches 14 and 15 .

\subsection{Glass Formulations for LAW Sub-Envelope A3}

LAW waste from tank AN-104 is designated as LAW Sub-Envelope A3. The current target glass composition for this waste is LAWA102. The sulfate concentration in this waste stream is the highest among all LAW A tanks and, consequently, the target glass composition contains only $14.5 \mathrm{wt} \% \mathrm{Na}_{2} \mathrm{O}$. Glass formulations prepared and characterized for LAW AN-104 waste are given in Table 3.7. The results of composition analysis by XRF are given in Table 4.6 and the results of DCP analyses are given in Table 4.11. The work on LAW Sub-Envelope A3 was all based on the glass composition LAWA102. One effort was designed to determine the effect of $\mathrm{Li}_{2} \mathrm{O}$ and $\mathrm{K}_{2} \mathrm{O}$ concentrations on glass properties, especially sulfate solubility. In these formulations, $\mathrm{Li}_{2} \mathrm{O}$ is used as an additive to improve the kinetics of sulfate incorporation as well as its solubility. However, $\mathrm{Li}_{2} \mathrm{O}$ additions can be detrimental since, while they reduce the likelihood of sulfate phase formation, should such a phase form, the lithium-containing 
sulfate phases in general tend to have lower melting points and are more corrosive to the refractories. Consequently, the current LAW glass formulation work included investigation of high-sulfate formulations containing potassium in place of lithium (LAWA118). Another variation investigated further increase in the calcium content (at the expense of iron) on sulfate solubility and crystallization on canister cooling. The canister cooling curve used in this work is given in Figure 1. This glass showed little, if any, crystallization indicating that the calcium levels can be increased. However, the improvement in sulfate solubility was small, in part because the LAWA102 glass already contains a significant amount of calcium (and lithium).

Another set of glasses was prepared by replacing all of the $\mathrm{Li}_{2} \mathrm{O}$ in LAWA102 with $\mathrm{K}_{2} \mathrm{O}$ on a mole basis. The resulting glass (LAWA120) contained $8.05 \mathrm{wt} \% \mathrm{~K}_{2} \mathrm{O}$. To compensate for the excess $\mathrm{K}_{2} \mathrm{O}$ on a mass basis, all other glass former additive oxides were reduced in proportion to their concentrations, as shown in Table 3.7. Another glass formulation (LAWA121) is similar to LAWA120 in composition but, in addition, all the iron oxide is replaced with $\mathrm{CaO}$; again, only a very modest increase in sulfate solubility was observed. Glass formulations LAWA122 and LAWA123 are similar in composition to LAWA120 and LAWA121, respectively, but with only $5.64 \mathrm{wt} \% \mathrm{~K}_{2} \mathrm{O}$. All four formulations were prepared and characterized at the nominal, and two excess sulfate levels, totaling 12 crucible melts. None of the glasses showed sufficient improvement over the properties of LAWA102 to warrant a change in the target glass formulation for LAW Sub-Envelope A3.

\subsection{Glass Formulations for LAW Sub-Envelope B1}

Waste from tank AZ-101 is designated as LAW Sub-Envelope B1. During Part B1, LAWB45 was selected as the target glass composition for this waste feed. The sulfate concentration in this waste feed is one of the highest among all of the Phase 1 LAW tanks and, as a consequence, the resulting glass contains only about $5 \mathrm{wt} \%$ waste $\mathrm{Na}_{2} \mathrm{O}$. The glass formulations that were investigated for LAW AZ-101 are given in Tables 3.8 to 3.12. The results of XRF analysis of the glass samples are given in Table 4.7 and the results of DCP analysis are given in Table 4.12. Glass development work concentrated on increasing the sulfate loading while minimizing the alkali content, and especially the $\mathrm{Li}_{2} \mathrm{O}$ content, of the glass. The formulation development was based on the results of earlier work [2] and LAWB45 was used as a starting point for the glass development work reported here. The components that were varied included calcium, iron, zinc, titanium, lithium, and potassium. Sulfate solubility and K-3 refractory corrosion were the properties that were most important in the selection of a target glass composition. The first set of glass formulations (LAWB60, LAWB69 to LAWB72, and LAWB77 to LAWB82) were prepared at a $\mathrm{Na}_{2} \mathrm{O}$ concentration of $6.62 \mathrm{wt} \%$ and a second set (LAWB83 to LAWB86) were targeted at a lower $\mathrm{Na}_{2} \mathrm{O}$ concentration of $5.47 \mathrm{wt} \%$, both from the LAW AZ-101 waste. Given the very low sodium content of these formulations (as a result of the low waste loading), another set of glasses was prepared and characterized to determine the effect of higher sodium and lower lithium on sulfate solubility. The higher sodium formulations, if selected for vitrification of LAW AZ-101, 
would require sodium additions as part of the glass formers. A third set of glasses was prepared and characterized, in which the lithium to sodium ratio was varied systematically in LAWB89 to LAWB95 (Table 3.12) to study its impact on K-3 corrosion. All three sets of glasses were formulated and characterized to optimize the target glass composition with respect to waste loading, sulfate solubility, and processability. In total, 71 crucible melts representing 21 formulations were prepared and tested as part of the glass development and optimization for LAW AZ-101. The large number of crucible melts resulted from preparation of the same target glass composition at different sulfate concentrations to determine the sulfate solubility.

Glasses with the highest lithium and calcium concentrations, such as LAWB69, showed the highest sulfate solubilities. However, as described previously [29], very high lithium concentrations in the glass have an adverse impact on K-3 refractory corrosion. Even though the neck and half-down corrosion (see Table 4.21) were not very high, highlithium glasses caused cracking of the K-3 refractory. Formulation LAWB83 was selected as the target composition for vitrification of LAW Sub-Envelope B1 waste feed from LAW AZ-101. LAWB83 has a reasonably high $\mathrm{Li}_{2} \mathrm{O}$ concentration of $4.31 \mathrm{wt} \%$ that is required to obtain the necessary sulfate solubility while not being so high as to cause excessive cracking of K-3 refractory. Based on these results, this formulation was successfully tested in the DM100 [30 and Pilot Melter [31] and was recommended for vitrification of the actual waste sample at PNWD [32].

\subsection{Glass Formulations for LAW Sub-Envelope B2}

Waste from tank AZ-102 is designated as LAW Sub-Envelope B2. The sulfate concentration in this waste stream is the highest among all Phase 1 LAW tanks. The waste contribution to the glass product, for this waste feed at a $\mathrm{Na}_{2} \mathrm{O}$ loading of $5.0 \mathrm{wt} \%$ before pretreatment, is given in Table 3.13. Glass development work concentrated on increasing the sulfate loading while decreasing the $\mathrm{Li}_{2} \mathrm{O}$ content of the glass, high levels of which can be detrimental to K-3 refractory cracking. The general approach paralleled that described above for LAW Sub-Envelope B1 (Section 3.4). Concentrations of glass former additives that were varied included calcium, iron, lithium, silicon, boron, and aluminum. The concentrations of the glass former additives were varied such that the resulting glasses all had approximately the same viscosity. Thirty crucible melts based on 12 different formulations (Table 3.14) were prepared as part of this glass development and optimization effort. Each of the formulations was prepared at different target sulfate concentrations to determine the effect of composition on sulfate solubility. Again, as previously seen for LAW AZ-101 formulations, increases in lithium and calcium lead to improved sulfate solubility. However, the very high lithium and calcium formulations (e.g. LAWB73 and LAWB74) resulted in excessive cracking of the K-3 refractory and, therefore, are not suitable target glass compositions for processing of the LAW AZ-102 waste [29]. One extreme example of K-3 refractory corrosion was LAWB62 with 11.96 $\mathrm{wt} \% \mathrm{CaO}$ and $5.79 \mathrm{wt} \% \mathrm{Li}_{2} \mathrm{O}$ : this glass has a very high sulfate solubility of about 1.8 wt $\%$ but the K-3 test coupon corroded to such an extent that the bottom of the coupon was severed and fell into the glass melt before the end of the 6-day testing period. VHT 
alteration of the high-lithium and high-calcium glasses, although still below the contractual limit, was among the highest of all glasses tested.

For the LAW AZ-102 waste stream, at the contract minimum waste loading of $5.0 \mathrm{wt} \% \mathrm{Na}_{2} \mathrm{O}$, the resulting glass will contain $1.28 \mathrm{wt} \% \mathrm{SO}_{3}$, as shown in Table 3.13. This LAW waste stream exceeds the contract limits with respect to sulfur to sodium ratio. Results of the above tests showed that even though a fully contractually compliant glass formulation can be developed for this waste stream, such a formulation would have undesirable characteristics with respect to K-3 refractory corrosion. Consequently, the Department of Energy-Office of River Protection (DOE-ORP) was advised through the WTP Project of the issues regarding treatment of this waste stream and the recommended options for treatment by either blending of this waste stream with another lower sulfur waste stream or lowering the waste loading [29]. The DOE-ORP elected to treat LAW AZ-102 waste separately at a lower waste loading. Based on this work, development of a glass formulation (LAWB96) at a lower waste loading to support DM100 and LAW Pilot melter tests was completed and is reported elsewhere [33]. LAWB96 is the glass composition currently selected for processing of LAW AZ-102 waste. In addition, another formulation LAWB88 was developed for vitrification and feed rheology testing of an actual sample of LAW AZ-102 at SRTC and is also reported separately [34].

\subsection{Glass Formulations for LAW Sub-Envelope C1}

Waste from tank AN-107 is designated as LAW Sub-envelope C1. The current target glass composition for this waste type is LAWC22. The sulfate concentration in this waste stream is the lowest of the Phase 1 Envelope $\mathrm{C}$ tanks and the resulting glass contains $12.26 \mathrm{wt} \%$ waste $\mathrm{Na}_{2} \mathrm{O}$ (14.42 wt $\% \mathrm{Na}_{2} \mathrm{O}$ including sodium from pretreatment). The target glass composition is given in Table 3.15 and XRF analysis results are given in Table 4.9. Review of the waste composition for LAW AN-107 showed that the existing glass composition, LAWC22, is still applicable. Therefore, no new glass formulations were tested. The composition of this waste is similar to that of LAW Sub-Envelope A3 waste, except for small differences in minor components and organics. One crucible melt of formulation LAWC22 was prepared and characterized as part of this work. As expected, properties such as PCT compared well with those previously reported [2] for this glass formulation but based on an earlier revision of the TFCOUP.

\subsection{Glass Formulations for LAW Sub-Envelope C2}

Waste from tank AN-102 is designated as LAW Sub-Envelope C2. The target glass composition for this waste feed was LAWC21. The sulfate concentration in this waste stream is the highest of the Phase 1 Envelope $\mathrm{C}$ tanks and the resulting glass contains $10.1 \mathrm{wt} \%$ waste $\mathrm{Na}_{2} \mathrm{O}$ (11.88 $\mathrm{wt} \% \mathrm{Na}_{2} \mathrm{O}$ including sodium from pretreatment). The target glass composition is given in Table 3.16. Glass formulation development work was directed towards improving the sulfate solubility without adversely affecting the K-3

refractory corrosion. The formulation development work was based on the results 
obtained earlier [2], with LAWC21 as the starting point. The target glass compositions are given in Tables 3.17 and 3.18. XRF and DCP analyses of the glasses are given in Tables 4.10 and 4.13, respectively. The first set of glass samples of the LAWC21 composition was prepared to assess the sulfate solubility of this reference formulation based on the waste composition given in TFCOUP Rev. 2. The measured $\mathrm{SO}_{3}$ content of the new glass agrees with the previously obtained value of $0.66 \mathrm{wt} \% \mathrm{SO}_{3}$. Another set of glasses was prepared with different amounts of calcium, zinc, and iron to determine their effect on sulfur solubility and K-3 refractory corrosion. The crystallization characteristics of the glasses were also measured to determine the extent to which higher $\mathrm{CaO}$ concentrations resulted in increased crystallization. LAWC21 showed little, if any, crystallization indicating that the calcium levels could be increased to potentially improve sulfate loading. In most of these formulations calcium and zinc concentrations were increased at the expense of iron. The sulfate solubility increased from about $0.66 \mathrm{wt} \%$ $\mathrm{SO}_{3}$ in LAWC21 with $6.4 \mathrm{wt} \% \mathrm{CaO}$ to about $1.1 \mathrm{wt} \% \mathrm{SO}_{3}$ in $\mathrm{LAWC} 28$ with $12.9 \mathrm{wt} \%$ $\mathrm{CaO}$. A total of 19 crucible melts representing 8 new formulations were prepared as part of the glass development and optimization work. Based on the results obtained, LAWC31 was selected as the revised target glass composition for LAW AN-102 waste. The new formulation contains slightly higher concentrations of calcium and zinc and the expense of some iron. Compared to LAWC21, LAWC31 has higher sulfate solubility and comparable K-3 corrosion characteristics ${ }^{3}$.

\footnotetext{
${ }^{3}$ As a result of a subsequently revised WTP waste basis which increased the sulfate to sodium ratio, this glass formulation work was extended to support melter changeover testing, which therefore employed a further revised formulation, LAWC35 [35].
} 


\section{SECTION 4.0 GLASS CHARACTERIZATION DATA}

Samples of the crucible melt glasses were tested with respect to the properties discussed in Section 2.0. The results of these tests are presented below.

\subsection{Chemical Composition}

The compositions of all of the glasses were determined by XRF and the results are given in Tables 4.1 to 4.10 . The target and analyzed glass compositions are generally in good agreement.

In order to determine sulfate solubility limits for different glass compositions, a number of samples were prepared at various target $\mathrm{SO}_{3}$ levels varying from 0.0 to 4.5 wt\%. An "Sx" suffix, with $\mathrm{x}=1,2,3,4$, at the end of a glass composition designation refers to a glass made with excess sulfate. Samples with an "S0" suffix were made with no sulfate in the target formulation. A "W" suffix in the sample designation indicates that the glass sample was washed to remove secondary and interstitial sulfate before composition analysis.

XRF analyses do not provide composition information for lithium and boron. Consequently, these were determined by DCP-AES. Glass samples were subjected to microwave-assisted total acid dissolution followed by solution analysis by DCP-AES. Results of the analyses are presented in Tables 4.11 to 4.13. These results are generally in agreement with the results of composition analysis by XRF and also provided confirmation of the boron and lithium concentrations in the glass samples.

\subsection{Viscosity and Electrical Conductivity}

The melt viscosity and electrical conductivity were measured in the range of $950^{\circ} \mathrm{C}$ to $1250^{\circ} \mathrm{C}$. The melt viscosity and electrical conductivity at standard temperatures are given in Tables 4.14 to 4.16. For convenience of comparison, these properties were interpolated at eight standard temperatures using the Vogel-Fulcher equation, which was fitted to the experimental data measured at a minimum of four different temperatures.

All glasses met the stated processing requirement of 10 to 150 Poise at $1100^{\circ} \mathrm{C}$. The viscosities of most of the glasses are between 30 and 90 Poise at $1150^{\circ} \mathrm{C}$. As expected, the glasses with the highest lithium, sodium, and calcium contents have the lowest viscosities. The viscosities of all but one of the glasses selected for waste processing (Table 5.1-5.7) are within the preferred range of $40-80$ Poise at $1150^{\circ} \mathrm{C}$. The viscosity of one of the glasses (LAWC22, Table 5.6) is reported as 39 Poise at $1150^{\circ} \mathrm{C}$, which is also very close to the preferred range (within the error of measurement). 
The majority of the glasses tested met the melt electrical conductivity requirement of $0.2-0.7 \mathrm{~S} / \mathrm{cm}$ at $1100-1200^{\circ} \mathrm{C}$. Some of the LAW Envelope B glasses with low $\mathrm{Na}_{2} \mathrm{O}$ contents showed low electrical conductivities in the range of 0.15 to $0.2 \mathrm{~S} / \mathrm{cm}$. Attempts to increase the electrical conductivity by addition of other alkalies and particularly lithium, in some cases resulted in undesirable corrosion characteristics towards K-3 refractory.

The viscosity and electrical conductivity of the glasses that were selected for melter tests and vitrification of radioactive samples fall into even narrower preferred ranges: The viscosity of these glasses typically ranges from 40 to 65 Poise at $1150^{\circ} \mathrm{C}$. The electrical conductivity typically ranged from $0.22 \mathrm{~S} / \mathrm{cm}$ for LAWB83 at $1150{ }^{\circ} \mathrm{C}$ to $0.56 \mathrm{~S} / \mathrm{cm}$ for LAWA 88 at $1150{ }^{\circ} \mathrm{C}[2]$.

\subsection{Product Consistency Test (PCT)}

The PCT results of the glass formulations, as measured per ASTM C1285 Method A ( 7 days at $\left.90^{\circ} \mathrm{C}\right)$ are presented in Table 4.17 to 4.19 . These tables include the leachate solution concentrations in ppm, the normalized glass mass loss (in $\mathrm{g} / \mathrm{L}$ and $\mathrm{g} / \mathrm{m}^{2}$ ), and the normalized loss rate $\left(\mathrm{g} / \mathrm{m}^{2} /\right.$ day). Glass ANL-LRM (Argonne National Laboratory LowActivity Reference Material), used by Argonne National Laboratory for a PCT roundrobin [36], was used as the reference glass with each test set.

The PCT results for all of the glasses developed in this work were below the contract limit of $2 \mathrm{~g} / \mathrm{m}^{2}$, by at least a factor of two in the case of boron and sodium, and by a factor of 10 or better in the case of silicon. The concentrations of boron and sodium in the leachates for most glasses were typically below one fifth of the contractual limit.

\subsection{Vapor Hydration Test (VHT)}

The VHT was performed on a total of 42 formulations, using a 24-day exposure for comparison to data collected during Part B1 [2], although some samples were also tested at the contract-specified duration of 7-days, both at $200^{\circ} \mathrm{C}$. One sample was tested for 48 days. The results are presented in Table 4.20. The results show that the selected glass compositions perform much better than the ILAW contract requirement. Among the glasses selected as target compositions for waste processing LAWC31 shows the highest VHT alteration rate.

\subsection{Liquidus Temperature and Crystallization Characteristics}

LAW glasses are designed to have a liquidus temperature of less than $950^{\circ} \mathrm{C}$. However, since LAW glasses generally show little or no crystallization at $950^{\circ} \mathrm{C}$ the samples were heat treated at lower temperatures. Heat treatments of glass samples for 20 
hours verified that the liquidus temperatures were below the heat treatment temperature of $850^{\circ} \mathrm{C}$. Selected samples were subjected to additional heat treatments at $700^{\circ} \mathrm{C}$ for 20 hours to determine their crystallization tendency during product cooling. Two of the selected formulations, LAWA44 and LAW88, were heat treated according to the canister cooling profile given in Figure 1, and characterized.

The majority of the heat-treated glasses remained homogeneous and did not show any crystallization. The results from characterization of heat-treated glasses are given in Table 4.21 for LAW Envelope B glasses, which are the only ones that showed any crystallization upon heat treatment. The crystallization characteristics of these glasses were determined using scanning electron microscopy coupled with energy dispersive x-ray spectroscopy (SEM/EDS), x-ray diffraction (XRD), and Optical Microscopy/ Image Analysis. In general, even for these glasses, the majority of the crystallization that was observed took place at the contact with the platinum crucible, which acted as a nucleation site. The total amount of crystals was low, typically less than 0.1 volume $\%$ after heat treatment for 20 hours at $850^{\circ} \mathrm{C}$.

\subsection{Refractory Corrosion}

Fifty-five of the glasses from this work were tested for their corrosion characteristics with respect to K-3 refractory. Refractory corrosion was an important criterion in the selection of target glass formulations. K-3 refractory cracking was the major factor limiting increases in the lithium concentration to improve sulfate solubility and, hence, waste loading in LAW Envelope B glasses. The results from 6-day K-3 coupon tests at $1208^{\circ} \mathrm{C}$ are given in Table 4.22 . The corrosion rate, as measured by loss of material over the test interval, was not unacceptably high for many of the glasses. However, cracking of the K-3 refractory coupon was a disqualifying factor for some of the LAW Envelope B glasses that were tested, as reported previously [29]. Optical micrographs of K-3 test coupons both before and after corrosion testing illustrating this behavior are given in Figure 2; cracking of the refractory exposed to LAWB64 glass can be seen in this figure.

\subsection{Glass Density and Glass Transition Temperature}

The glass transition temperatures and densities of selected glasses are presented in Tables 4.23 and 4.24, respectively. As previously observed [2], for most LAW glasses, these properties show relatively little change with glass composition. The glass transition temperatures are in the range of 472 to $512^{\circ} \mathrm{C}$. The glass densities are about $2.7 \mathrm{~g} / \mathrm{cc}$, which is similar to the values reported previously [2]. 


\subsection{Toxicity Characteristic Leaching Procedure (TCLP)}

TCLP results for the glasses selected for processing of the different waste streams are given in Table 4.25. As evident from the Table, TCLP results for LAW glasses are well below the Universal Treatment Standard (UTS) limits. This is as expected because the LAW waste streams contain little or no hazardous components.

\subsection{Redox Evaluation by Mössbauer Spectroscopy}

The redox states of several of the glass samples were determined by Mössbauer spectroscopy; typical spectra are given in Figure 3. The spectra show only two peaks, which are characteristic of $\mathrm{Fe}^{3+}$. The $\mathrm{Fe}^{2+}$ peaks are absent from these spectra indicating that the glasses are fully oxidized (less than $1 \% \mathrm{Fe}^{2+}$ ). This is as expected because the crucible melts of the glasses were prepared in air without the addition of reductants.

\subsection{Other Requirements}

Construction of TTT diagrams for the target LAW glass formulations (Contract Specification 2.2.2.6.3); leachability index per ANSI/ANS-16.1 (Contract Specification 2.2.2.17.1); compressive strength (Contract Specification 2.2.2.18); and compressive strength after thermal, radiation, biodegradation, and immersion per ANSI/ANS 16.1 (Contract Specification 2.2.2.19), were not part of the current test scope [9]. 


\section{SECTION 5.0 SUMMARY AND CONCLUSIONS}

Glass formulation development and testing was performed to address eight of the Phase 1 LAW tank wastes: AP-101, AZ-101, AZ-102, AN-102, AN-104, AN-105, AN-107 and AW-101. The work concentrated on developing contractually compliant glasses that are suitable for processing in the joule-heated DuraMelter units that form the basis of the WTP LAW vitrification systems. The work was based on the waste composition data given in TFCOUP Rev. 2 [5] except for LAW AP-101, for which the actual waste sample characterization data [8] was used. Information from the earlier glass formulation work $[1,2]$ was used to guide the current work. Data from two companion tasks, LAW glass formulations for melter testing [20] and LAW glass formulations to support active waste testing [21], were also used to complement the data generated during this work and all three tasks were interactive.

Glass former additives are added to the waste to produce a glass with the desired properties. The types and amounts of glass former additives are adjusted to control the properties of the glass melt and glass product. During the present work, the same 10 glass former oxides as used in Part $\mathrm{B} 1$ were used: $\mathrm{Al}_{2} \mathrm{O}_{3}, \mathrm{~B}_{2} \mathrm{O}_{3}, \mathrm{CaO}, \mathrm{Fe}_{2} \mathrm{O}_{3}, \mathrm{Li}_{2} \mathrm{O}, \mathrm{MgO}, \mathrm{SiO}_{2}$, $\mathrm{TiO}_{2}, \mathrm{ZnO}$, and $\mathrm{ZrO}_{2}$. Earlier studies [1,2] investigated a wide variety of alternative additives and consequently, no new potential additives were investigated in the present work. Furthermore, the WTP has designed the chemical handling and feed make-up system based on using raw materials of the above oxides.

Contractually compliant glass formulations that also meet the processing requirements were identified for all waste streams except LAW AZ-102. As explained below, a target glass formulation at a lower waste loading was developed, as directed by DOE-ORP through the WTP Project.

The ratio of sulfate to sodium in the LAW waste stream is the dominant waste loading limiting criterion. Consequently, during Part B1, LAW waste streams were conveniently divided into seven Sub-Envelopes based on the sulfate to sodium ratios in the Envelope A, B, and C waste streams [2]. That division was carried over to the current work and results for each Sub-Envelope are summarized below.

LAW Envelope A: The majority of the LAW waste from double shell tanks corresponds to this envelope. The formulation development work for LAW Envelope A was divided into three subsets as given below.

- Sub-Envelope A1: These waste streams are characterized by low sulfate and low potassium, and waste loading is limited by sodium content of the glass. In order to maintain acceptable refractory corrosion characteristics, waste loading is limited to about $20 \mathrm{wt} \% \mathrm{Na}_{2} \mathrm{O}$ in the product glass. The glass LAWA44 (Table 5.1), which had been selected for this waste type in Part B1 
[2] was found to be acceptable for the first batch of LAW AN-105. This formulation has now been tested in the DM100 melter [26] and at large-scale in the LAW Pilot melter [27]. The second batch of LAW AN-105 from dissolved saltcake has a much higher sulfate concentration and LAWA44 may not be a suitable target glass composition. A target formulation for the second batch of LAW AN-105 was not selected because this work was terminated by the WTP Project prior to its completion. However, based on the data from LAW glass formulation development work to-date, it will be possible to identify a compliant target formulation for the higher-sulfate waste with limited additional work.

- Sub-Envelope A2: These waste streams are characterized by low sulfate and high potassium, and waste loading is limited by the total alkali (sodium and potassium) concentration in the glass. The glass LAWA88 (Table 5.2) previously selected for this waste type is still viable for tank AW-101 LAW. The potassium concentration in the LAW AP-101 waste stream has increased significantly according to waste characterization data [8]. Consequently, LAWA88 is no longer an acceptable glass formulation for the vitrification of LAW AP-101 because the very high alkali content results in unacceptable K-3 refractory corrosion. During the current work, a new glass formulation, LAWA126 (Table 5.3), at a lower waste loading was selected as the target glass formulation for LAW AP-101.

- Sub-Envelope A3: These waste streams have the highest sulfate concentration among all Phase 1 Envelope A wastes. The waste loading is limited by the sulfate content of the glass and, consequently, the corresponding $\mathrm{Na}_{2} \mathrm{O}$ concentration falls close to the contract minimum of $14.0 \mathrm{wt} \%$. Variations around the glass LAWA102 (Table 5.4), which was selected for this waste type previously [2], were investigated but the results did not warrant revision of this target composition for LAW tank AN-104 waste. Minor changes to the waste composition can be compensated by varying the relative concentrations of the glass former additives.

LAW Envelope B: Of the waste streams that were investigated in this work, AZ-101 and AZ-102 LAW are the only Envelope B streams. During Part B1, the feed delivery plan included blending of these two waste streams. They have the highest sulfate concentrations among all early LAW feeds and therefore the waste loadings are low. Per the current plan, these two waste streams will be delivered separately to the WTP. In addition, the new waste composition data [5] indicated an increase in the sulfate concentration for these waste streams. In fact, LAW AZ-102, which has the highest sulfate concentration, is no longer a contractually-compliant waste feed. Due to all of the above factors, a large part of the current baseline glass formulation work was directed towards identifying target glass formulations for LAW AZ-101 and AZ-102 when processed separately and with higher sulfate concentrations. The work focused on adjusting the glass former additives to maximize sulfate loading without adversely affecting refractory corrosion characteristics of the resulting glass. 
- Sub-Envelope B1: LAW AZ-101 is designated as LAW Sub-Envelope B1. The glass formulation development was directed towards improving the sulfate solubility of the target glass. Results of previous and current work showed that increasing the $\mathrm{CaO}$ and $\mathrm{Li}_{2} \mathrm{O}$ content of the glasses increased the sulfate solubility. However, very high $\mathrm{Li}_{2} \mathrm{O}$ concentrations ( $>4.5 \mathrm{wt} \%$ ) resulted in excessive cracking of the K-3 refractory coupons, which is a concern with respect to the life of the LAW melter. Based on these considerations and the results from this work, LAWB83 (Table 5.5), with a $\mathrm{Li}_{2} \mathrm{O}$ concentration of $4.31 \mathrm{wt} \%$ in the glass, was selected as the target glass formulation for LAW AZ-101. Glasses with higher lithium contents and correspondingly higher sulfate solubilities were rejected as target formulations due to unacceptable K-3 refractory cracking.

- Sub-Envelope B2: LAW AZ-102 is designated as LAW Sub-Envelope B2. This has the highest sulfate content among all LAW streams studied. The sulfate to sodium ratio for this waste stream exceeds the contractual limits. Glass formulation development work for LAW AZ-102 paralleled that for LAW AZ-101. It is possible to identify fully compliant glass formulations for this waste stream. However, due to the very high sulfate content, it became necessary to add high concentrations of $\mathrm{Li}_{2} \mathrm{O}$, which resulted in the glass melt having unacceptable K-3 corrosion characteristics. The WTP Project informed DOE-ORP of this issue with a recommendation to either blend the waste or to reduce the waste loading. DOE-ORP elected to reduce the waste loading and a corresponding glass formulation, LAWB96, was developed to support subsequent DM100 and LAW Pilot melter testing. The development of LAWB96 was performed under a different task [20,33] based on the results from the present work and the results will therefore be reported separately.

LAW Envelope C: These wastes contain significant amounts of sulfate and waste organics. The wastes are divided into two sub-sets based on their sulfate to sodium ratio, which is the waste loading limiting criterion.

- Sub-Envelope C1: LAW Envelope C wastes that contain lower amounts of sulfate are classified as Sub-Envelope C1. The sulfate levels are similar to those found in tank AN-107 and they are comparable to Sub-Envelope A3 in composition. The formulation LAWC22 (Table 5.6) that was selected earlier for LAW tank AN-107 was found to be still applicable.

- Sub-Envelope C2: These LAW Envelope C wastes contain higher amounts of sulfate and therefore the waste $\mathrm{Na}_{2} \mathrm{O}$ loading in the resulting glass is close to the contract minimum of $10.0 \mathrm{wt} \%$. LAW AN-102 is classified as LAW SubEnvelope C2. The sulfate solubility of the earlier target formulation, LAWC21 (Table 5.7), was judged to be too low for the new waste composition basis [5]. Accordingly, glass formulation development was conducted to identify a revised formulation with higher sulfate solubility. Based on the results of the 
work, the target formulation for this waste was revised to LAWC31 that is higher in calcium and zinc and lower in iron compared to LAWC21 and which has a higher sulfate solubility.

Optimization of glass composition to maximize sulfate solubility was a key aspect of the baseline glass formulation work. The results of sulfate incorporation measurements from the present work and from previous work [1, 2], as well as formulations used in melter tests, are presented in Figure 4. In this figure, sulfate content is plotted as a function of $\mathrm{Na}_{2} \mathrm{O}$ concentration in the glass. $\mathrm{Na}_{2} \mathrm{O}$ concentrations much above $20 \mathrm{wt} \%$ are not tested because at higher concentrations the glass melts tend to have unacceptable processing properties, such as refractory corrosion. The achievable sulfate content in the glasses tested decreases as sodium concentration in the glass increases. The data support the use of a $\mathrm{Na}_{2} \mathrm{O} \times \mathrm{SO}_{3}$ product rule as was previously postulated [37] (such as the "rule of five") to provide a guide for prediction of the practically achievable waste loading of sodium and sulfur bearing waste streams. As can be seen from Figure 4, a $\mathrm{Na}_{2} \mathrm{O} \times \mathrm{SO}_{3}$ value of greater than 5 is achievable for a range of $\mathrm{Na}_{2} \mathrm{O}$ and $\mathrm{SO}_{3}$ values. However, in the low $\mathrm{Na}_{2} \mathrm{O}$ and high $\mathrm{SO}_{3}$ region, it is more difficult to exceed or even achieve this value due to other glass formulation considerations. Refinement of this relationship over a range of relevant $\mathrm{Na}_{2} \mathrm{O}$ and $\mathrm{SO}_{3}$ values is part of future work scope. At high $\mathrm{SO}_{3}$ concentrations, it is necessary to add large amounts of $\mathrm{Li}_{2} \mathrm{O}$ to achieve the desired solubility. However, this leads to unacceptable cracking of K-3 refractory. Therefore, even though higher $\mathrm{SO}_{3}$ concentrations are possible for the low $\mathrm{Na}_{2} \mathrm{O}$ region in Figure 4, such glass formulations are not be suitable based on melter life considerations. It should also be noted that under the dynamic conditions prevailing in an actual melter, sulfate separation occurs before the underlying melt reaches sulfate saturation $[14,15,37,38]$. Consequently, the practically achievable sulfate content will always be less than the solubility limit. For this reason, the melter formulations shown in Figure 4 fall below the highest achievable sulfate concentrations.

Estimates of the sulfate to sodium ratios for 28 double-shell tanks and 149 singleshell tanks are given in Appendix A and shown graphically in Figures 5 and 6 . These are based on the composition data presented in TFCOUP Rev. 3A [7] and were generated with the assumption that $50 \%$ of sodium and $75 \%$ of the sulfate in the solid fraction would be returned to the liquid feed. As can be seen from the figures, sulfate to sodium ratios for the vast majority of the LAW waste streams are within the limits already studied. From Figure 4, it is evident that glasses that cover almost the entire range of these sulfate-to-sodium ratios have already been prepared and tested. The current and previous work has also identified preferred methods to optimize glass compositions via glass former additives to tailor the glass composition to a particular waste stream. As a result, the currently existing data base and information provides the foundation for the identification of target glass formulations for new waste streams. 


\section{SECTION 6.0 \\ EXPERIMENTAL METHODS}

A short description of the experimental methods and equipment that were used for this work is provided below. The techniques are described in detail in controlled technical procedures [39] that form part of the VSL QA program [40].

\subsection{Glass Preparation}

The glasses were prepared from reagent grade or higher purity chemicals to produce a batch size of approximately $400 \mathrm{~g}$ according to VSL standard operating procedures. Crucible melts were prepared by melting the appropriate combination of well-mixed chemicals at $1200^{\circ} \mathrm{C}$ for 75 minutes in a platinum, platinum-gold, or platinum-rhodium crucible. Mixing of the melt was accomplished with a platinum stirrer beginning 15 minutes after the start of melting and continuing for the next 60 minutes, until the end of melting. The molten glass was poured onto a graphite plate to cool, and the resulting glass was then distributed for analyses.

\subsection{Compositional Analysis}

Glass samples for analysis were subjected to microwave-assisted total acid dissolution in Teflon vessels according to VSL standard operating procedures. Twenty milliliters of a 1:5 mixture of concentrated $\mathrm{HF}: \mathrm{HNO}_{3}$ were diluted to $50 \mathrm{ml}$ and used for the dissolution. This procedure is similar to the ASTM Test Method C1412-99, which also employs a mixture of concentrated $\mathrm{HF}$ and $\mathrm{HNO}_{3}$ in microwave digestion of pulverized glass samples; supplemental use of $\mathrm{HCl} / \mathrm{H}_{3} \mathrm{BO}_{3}$ is not included in the VSL procedure since boron is normally one of the analytes. The resulting solutions were analyzed by direct current plasma atomic emission spectroscopy (DCP-AES) for all constituents except sulfur, for which Dionex Ion Chromatography was used. However, the primary method used for sulfur analysis was X-ray fluorescence (XRF) on powdered glass samples, which also provides corroborative data for the other analytes. An ARL 9400 wavelength dispersive XRF spectrometer was used for this purpose.

\subsection{Viscosity}

The melt viscosity, $\eta$, was measured using a Brookfield viscometer. Measurements were performed in the temperature range of $950-1250^{\circ} \mathrm{C}$, and the data interpolated to standard temperatures using the Vogel-Fulcher equation: $\ln \eta=[\mathrm{A} /(\mathrm{T}-$ $\mathrm{T}_{\mathrm{o}}$ )] $+\mathrm{B}$, where $\mathrm{A}, \mathrm{B}$, and $\mathrm{T}_{\mathrm{o}}$ are fitting parameters. The equipment was calibrated at room temperature using standard oils of known viscosity, and then checked at $950-1250^{\circ} \mathrm{C}$ using a National Institute of Standards and Technology (NIST) standard reference glass (SRM 711). Both precision and accuracy of the viscosity measurements are estimated to be within \pm 15 relative $\%$. 


\subsection{Electrical Conductivity}

The electrical conductivity, $\sigma$, of each glass was determined by measuring the resistance of the glass melt as a function of frequency using a calibrated platinum/rhodium electrode probe attached to a Hewlett-Packard model 4194A impedance analyzer. Measurements were performed over similar temperature ranges to those employed for the melt viscosity measurements. The results were extrapolated to zero frequency to obtain the DC electrical conductivity. The electrical conductivity data were then interpolated to standard temperatures using the Vogel-Fulcher equation: $\ln \sigma=\left[\mathrm{A} /\left(\mathrm{T}-\mathrm{T}_{\mathrm{o}}\right)\right]+\mathrm{B}$, where $\mathrm{A}, \mathrm{B}$ and $\mathrm{T}_{\mathrm{o}}$ are fitting parameters. Estimated uncertainties in the electrical conductivity measurements are \pm 20 relative $\%$.

\subsection{Liquidus Temperature and Crystallinity}

An upper bound on the liquidus temperature of the glass was determined by heat treatment followed by analysis using optical and/or electron microscopy. Samples of about $500 \mathrm{mg}$ were heated in a platinum, platinum-gold, or platinum-rhodium crucible at a pre-melt temperature of $1200^{\circ} \mathrm{C}$ for one hour to destroy any pre-existing nuclei, followed by heat treatment at $850^{\circ} \mathrm{C}$ or $700^{\circ} \mathrm{C}$; heat treatment times of 20 hours were used. The heat-treated glass was then quenched by submerging the outside of the crucible in cold water. This quenching freezes in the phase assemblage in equilibrium with the melt at the heat treatment temperature. If no crystals are observed in the cooled glass, then it is inferred that no crystals are present at the heat treatment temperature and that the liquidus temperature is below the heat treatment temperature. The LAW glasses are designed to have a liquidus temperature of less than $950^{\circ} \mathrm{C}$. In view of the generally low liquidus temperatures for the LAW glasses [2], heat treatments were done at lower temperatures $\left(850^{\circ} \mathrm{C}\right.$ and $\left.700^{\circ} \mathrm{C}\right)$.

\subsection{Secondary Phases}

Scanning electron microscopy (SEM) was used to characterize the microstructure of the glasses and energy dispersive x-ray spectroscopy (EDS) was used to analyze the glassy and crystalline phases that were observed. Typical magnifications used ranged from $25 \times$ to $5,000 \times$.

Digital imaging and analyses permit determination of the volume fractions of crystalline phases in both as-melted and heat-treated glasses. The accuracy of the volume percentage determinations is limited by uniformity of distribution and imaging characteristics of a phase. The accuracy of the volume percentage determination is estimated to be within $20 \%$ relative.

Optical microscopy with image analysis and quantitative $\mathrm{x}$-ray diffraction were used in combination with the SEM/EDS measurements. 


\subsection{Product Consistency Test (PCT)}

The product consistency test (PCT; ASTM C 1285-94) is used to evaluate the relative chemical durability of glasses by measuring the concentrations of the chemical species released from 100-200 mesh crushed glass $(75-149 \mu \mathrm{m})$ to the test solution (deionized water in this case). PCT tests on the LAW glasses were performed at $90^{\circ} \mathrm{C}$, in accordance with the current RPP-WTP contract requirement. The ratio of the glass surface area to the solution volume for this test is about $2000 \mathrm{~m}^{-1}(10 \mathrm{~g}$ of $100-200 \mathrm{mesh}$ glass is immersed in $100 \mathrm{ml}$ deionized water). All tests were conducted in triplicate, in 304L stainless steel vessels, and in parallel with a standard glass included in each test set. The internal standard was the ANL-LRM reference glass [36], which has undergone round-robin testing. The leachates are sampled at predetermined times, the first of which is seven days. One milliliter of sampled leachate is mixed with $20 \mathrm{ml}$ of $1 \mathrm{M} \mathrm{HNO}_{3}$ and the resulting solution is analyzed by DCP-AES; another $3 \mathrm{ml}$ of sampled leachate is used for $\mathrm{pH}$ measurement.

\subsection{Vapor Hydration Test (VHT)}

The vapor hydration tests are run in Parr series 4700 screw-cap pressure vessels made of 304L stainless steel and having either 22 or $45 \mathrm{ml}$ capacity in accordance with the procedure given in Appendix A of the PSWP [22]. Glass coupons are fashioned about 5 to $10 \mathrm{~mm}$ square, about $2 \mathrm{~mm}$ thick, and with one cut and one fracture surface face. A hole approximately $1.6 \mathrm{~mm}$ in diameter is drilled near one corner of the coupon to allow it to be suspended from a hanger made of 24 gauge stainless steel wire. Dimensional measurements are made to permit calculation of the area and the coupon is weighed before and after the VHT on a balance having a resolution of $100 \mu \mathrm{g}$. The coupon is suspended vertically from the hanger in the pressure vessel and enough deionized water is added to the vessel to saturate the volume at the test temperature, $200^{\circ} \mathrm{C}$, and to allow for a non-dripping layer covering the coupon. The pressure vessels are flushed with argon, sealed, weighed, and placed in an oven held at $200^{\circ} \mathrm{C}$. The temperature is monitored continuously with an independent thermocouple. At the completion of the test, the pressure vessels are removed and immediately partially immersed in an ice/water bath to condense the water vapor near the bottom of the vessel. Once cool, the vessels are weighed and opened, and then the coupons are removed and weighed. If the difference in the mass of the sealed pressure vessel before and after the test indicated a water loss in excess of $50 \%$ of the original amount, the test results are discarded. Otherwise, the coupons are then examined using low-power optical microscopy and an X-ray diffraction pattern is taken directly off the surface of the coupon. Next, the coupons are sectioned and the pieces mounted separately to allow for SEM examination both of the cross section of the leached coupon and the leached surface itself. For consistency with existing data, the nominal test duration was 24 days; however, a shorter duration (7 days) was also used for selected glasses. 


\subsection{Glass Density}

Density measurements were made on 5 to 15 grams of crushed glass using the pycnometric method described in ASTMD 854-83. Densities of two NIST glasses (Lead Silicate Glass \#1827a and Soda Lime Glass \#1826b) were measured as calibration checks. To confirm the reproducibility of the results, triplicate analyses of ten percent of the samples were performed, as required by the procedure.

\subsection{Glass Transition Temperature}

The glass transition temperature was measured by differential thermal analysis (DTA) with respect to a reference material that has no transitions in the region of interest (e.g., alumina). About $30 \mathrm{mg}$ of powdered glass sample $(<75 \mu \mathrm{m}$ particle size $)$ were analyzed with a Perkin Elmer differential thermal analyzer DTA7 coupled to a TAC $7 / \mathrm{DX}$ controller. The sample is heated from $300^{\circ} \mathrm{C}$ to $700^{\circ} \mathrm{C}$ at a rate of $10^{\circ} \mathrm{C} / \mathrm{min}$ while the temperatures of the sample and reference are monitored. During the heating, any endothermic or exothermic transitions in the sample are reflected as a simultaneous temperature difference between the sample and the reference. The glass transition temperature was determined as the extrapolated onset temperature in a manner similar to that described in ASTM E 1356-91 (Standard Test Method for Glass Transition Temperatures by Differential Scanning Calorimetry or Differential Thermal Analysis). Extrapolations from the first baseline deviation temperature and from the midpoint temperature, for which the point of inflection is used, are drawn. The glass transition temperature is taken as the temperature at the intersection of the two extrapolations.

\subsection{Toxicity Characteristics Leaching Procedure (TCLP)}

TCLP was performed at the VSL, according to SW-846 Method 1311, on crushed glass $\left(<3 / 8^{\prime \prime}\right)$ in a sodium acetate buffer solution after 18 hours at $22^{\circ} \mathrm{C}$ with constant end-over-end agitation. A mass of about 100 grams of glass was leached in 2 liters of TCLP extract, according to the extraction method for non-volatiles. The surface area to volume ratio for this test is about $20 \mathrm{~m}^{-1}$, which is about two orders of magnitude lower than that in the PCT. Since important glass matrix constituents are not determined by the standard TCLP analysis methods, these data were supplemented as necessary by data obtained by DCP-AES according to VSL standard operating procedures. 


\section{SECTION 7.0 QUALITY ASSURANCE}

This work was conducted under an NQA-1 (1989) and NQA-2a (1990) Part 2.7 based quality assurance program that is in place at the VSL. This program is supplemented by a Quality Assurance Project Plan for RPP-WTP work [40] that is conducted at VSL. Test and procedure requirements by which the testing activities are planned and controlled are also defined in this plan. The program is supported by VSL standard operating procedures that were used for this work [39]. This work was not subject to DOE/RW-0333P. This work was not subject to the requirements of WTP QAPjP [41] for environmental regulatory data. 


\section{SECTION 8.0 REFERENCES}

[1] "Glass Formulation and Testing with TWRS LAW Simulants," I.S. Muller and I.L. Pegg, Vitreous State Laboratory, Washington, D.C., January 16, 1998.

[2] "Glass Formulation and Testing with RPP-WTP LAW Simulants," I.S. Muller, A.C. Buechele and I.L. Pegg, VSL-00R3560-2, Vitreous State Laboratory, Washington, D.C., February 232001.

[3] "LAW Baseline Glass Formulation Testing", I.S. Muller and I.L. Pegg, Test Plan, VSL-01T5600-3, Rev. 1, Vitreous State Laboratory, January 21, 2002.

[4] "Low Activity Waste Feed staging for Case 3S6D" in "Tank Farm Contributor Operation and Utilization Plan", Kirkbride R.A. et al., HNF-SD-WM-SP-012, Rev. 2.

[5] "Low Activity Waste Feed staging for Case 3S6E" in "Tank Farm Contributor Operation and Utilization Plan", Kirkbride R.A. et al., HNF-SD-WM-SP-012, Rev. 2, 4/19/00.

[6] "Data Extracted from Appendices C and D" in "Tank Farm Contributor Operation and Utilization Plan", Kirkbride R.A. et al., HNF-SD-WM-SP-012, Rev. 3, email transmission from Randy A Kirkbride of Numatec Hanford Corporation on $10 / 2 / 01$.

[7] "Data Extracted from Appendices C and D" in "Tank Farm Contributor Operation and Utilization Plan", Kirkbride R.A. et al., HNF-SD-WM-SP-012, Rev. 3A.

[8] Fiskum, S.K., et.al., "Inorganic, Radioisotopic, and Organic Analysis of 241-AP101 Tank Waste”, PNNL-13354 (Formerly PNWD-3046), PNNL, October 2000.

[9] "Baseline LAW Glass Formulation,” D. Swanberg, RPP-WTP Test Specification, No. 24590-LAW-TSP-RT-01-009, Rev. 0., September 20, 2001

[10] “LAW Glass Property Composition Modeling," H. Gan and I.L. Pegg, Test Plan, VSL-02T4800-1, Rev. 1, Vitreous State Laboratory, April 16, 2002.

[11] "Test Matrix to Support Property-Composition Model Development for RPPWTP LAW Glasses," S.K. Cooley, G.F. Piepel, H. Gan, I.S. Muller, and I.L. Pegg, Data Summary Report, VSL02S4600-3, Rev. 1 (Draft), Vitreous State Laboratory, February 14, 2003. 
[12] "Basis of Design”, BNFL Report, DB-W375-EG00001, Rev. 0, November 23, 1998.

[13] “Basis of Design”, RPP-WTP Project, DB-W375-EG-00001, 12/28/2000.

[14] "Melter Tests with LAW Envelope A and C Simulants to Support Enhanced Sulfate Incorporation,” K.S. Matlack, S. Morgan, and I.L. Pegg, VSL-01R3501-2, $1 / 26 / 01$.

[15] "Melter Tests with LAW Envelope B Simulants to Support Enhanced Sulfate Incorporation," Final Report, K.S. Matlack, S. P. Morgan, and I.L. Pegg, VSL00R3501-1, Rev. 0, 11/27/00.

[16] "LAW Pilot Melter and DM-100 Sub-Envelope Changeover Testing," E.V. Morrey, WTP Test Specification, 24590-LAW-TSP-RT-02-012, Rev. 0, August $13,2002$.

[17] “Updated Instructions for ILAW Regulatory Spike Testing," WTP Memorandum, C.A. Musick to I.L. Pegg, CCN \#049799, January 9, 2003.

[18] "Corrosion of K-3 Refractory and Metal Alloys in RPP-WTP LAW Glasses," H. Gan, X. Lu, I. Vidensky, and I.L. Pegg, Final Report, VSL-01R3540-1, March 16, 2001.

[19] “Corrosion Testing of Bubbler Materials for LAW Vitrification," I. Vidensky, H. Gan, W. Gong, and I.L. Pegg, Test Plan, VSL-02T3430-1, Rev. 0, Vitreous State Laboratory, March 27, 2003.

[20] "LAW Glass Formulation to Support Melter Runs with Simulants", I.S. Muller and I.L. Pegg, Test Plan, VSL-01T5600-1, Rev. 1, Vitreous State Laboratory, November 15, 2001.

[21] "LAW Glass Formulation to Support Actual Waste Testing", I.S. Muller and I.L. Pegg, Test Plan, VSL-01T5600-2, Rev. 1, Vitreous State Laboratory, December 3,2001 .

[22] "Products and Secondary Wastes Plan (PSWP)," RPP-WTP Project, PL-W375TE00001, Rev. 0, 4/12/2000.

[23] "RPP-WTP Pilot Melter Off-Gas System Sampling (High Sulfate Envelope C) Test Results Report," GTS Duratek, Inc., TRR-PLT-56B, 10/11/00.

[24] "RPP-WTP Pilot Melter Envelope A and C Throughput Test Results Report," GTS Duratek, Inc., TRR-PLT-54, 10/13/00. 
[25] "RPP-WTP Pilot Melter Envelope B Throughput Test Results Report," GTS Duratek, Inc., TRR-PLT-57, 10/13/00.

[26] "Compositional Variation Tests on DuraMelter 100 with LAW Sub-Envelope A1 Feed (LAWA44 Glass) in Support of the LAW Pilot Melter," K.S. Matlack, W. Gong, and I.L. Pegg, Final Report, VSL-02R62N0-4, Rev. 1, Vitreous State Laboratory, November 8, 2002.

[27] "RPP-WTP Pilot Melter Sub-Envelope A1 Variation Test Results Report, Duratek, Inc., TRR-PLT-071 (draft).

[28] "LAW Glass Formulation to Support AP-101 Actual Waste Testing," I.S. Muller and I.L. Pegg, Vitreous State Laboratory, Washington, D.C., VSL-02R3470-2, Rev. A, 1/24/03.

[29] "Enhanced K-3 Refractory Corrosion with LAW AZ-102 Glass Formulations, Summary Report", I. S. Muller, H. Gan, A.C. Buechele, and I L. Pegg, VSL02S4600-1 rev.0, June 25, 2002.

[30] "Compositional Variation Tests on DuraMelter 100 with LAW Sub-Envelope B1 Feed in Support of the LAW Pilot Melter," K.S. Matlack, W. Gong, and I.L. Pegg, Final Report, VSL-02R62N0-5, Rev. A, Vitreous State Laboratory, October $18,2002$.

[31] "RPP-WTP Pilot Melter Sub-Envelope B1Variation Test Results Report, Duratek, Inc., TRR-PLT-074 (draft).

[32] "LAW Glass Formulation to Support AZ-101 Actual Waste Testing," I.S. Muller and I.L. Pegg, Final Report, VSL-03R3470-3, Rev. A, Vitreous State Laboratory, Washington, D.C., March 20, 2003.

[33] “Test Plan: Test on DuraMelter 100 with LAW Sub-Envelope B2 Feed in Support of the LAW Pilot Melter," K.S. Matlack, I.S. Muller and I.L. Pegg, Vitreous State Laboratory, Washington, D.C., VSL-02T62N0-5, Rev.0, 11/26/02.

[34] "LAW Glass Formulation to Support AZ-102 Actual Waste Testing," I.S. Muller and I.L. Pegg, Vitreous State Laboratory, Washington, D.C., VSL-02R3470-1, Rev. A, 1/10/03.

[35] "DuraMelter 100 Sub-Envelope Changeover Testing Using LAW Sub-Envelope A3 and C2 Feeds in Support of the LAW Pilot Melter," K.S. Matlack and I.L. Pegg, Data Summary Report, VSL-03S3410-3, Rev. 0, Vitreous State Laboratory, Washington, D.C., April 3, 2003. 
[36] "Results or Round Robin Testing of TFA LAW Reference Glass LRM," S.S. Fu, S.T. Lai, I.S. Muller, F. Perez Cardenas and I.L. Pegg, VSL-00R3620-1, Rev.0, 4/19/00.

[37] "Summary of Preliminary Results on Enhanced Sulfate Incorporation During Vitrification of LAW Feeds", I.L. Pegg, H. Gan, I.S. Muller, D.A. McKeown, and K.S. Matlack, VSL-00R3630-1, April 52000.

[38] "Sulfur Incorporation in Waste Glass Melts of Various Compositions," W.K. Kot, H. Gan, and I.L. Pegg, Ceramic Transactions, vol. 107, pp. 441, Eds. G.T. Chandler and X. Feng, American Ceramic Society, 2000.

[39] "Master List of Controlled VSL Manuals and Standard Operating Procedures in Use," QA-MLCP, Rev. 5, November 7, 2002.

[40] "Quality Assurance Project Plan for RPP-WTP Support Activities Conducted by VSL," Vitreous State Laboratory, QAPP Rev. 4, 8/2/2001.

[41] "Quality Assurance Project Plan for Testing Programs Generating Environmental Regulatory Data,” D. B. Blumenkranz, PL-24590-QA00001, Rev. 0, Bechtel National Inc., Richland, WA, June 7, 2001.

[42] Contract No. DE-AC27-01RV14136; Section C: Statement of Work, Specification 7 Low-Activity Waste Envelope Definition, December 2000.

[43] "Interface Control Document for Waste Treatability Samples," BNFL-5193-ID23, Rev. 4, June 22, 2000.

[44] Nelson, J, D Swanberg, C Roberts. 2003. ILAW Product Compliance Plan, 24590-WTP-PL-RT-03-001, River Protection Project, Waste Treatment Plant. 
Table 1.1. Summary of ILAW Requirements.

\begin{tabular}{|c|c|c|c|c|}
\hline \begin{tabular}{|c|} 
Specification source in contract \\
[42] unless specified
\end{tabular} & Contracting Requirements & \multicolumn{3}{|c|}{ Required limit of given specification } \\
\hline 2.2 .2 .2 & $\begin{array}{c}\text { Waste Loading } \\
\text { (based on } \mathrm{Na}_{2} \mathrm{O} \text { wt\%) }\end{array}$ & $\begin{array}{l}>14 \% \\
\text { LAWA }\end{array}$ & $\begin{array}{c}>5 \% \\
\text { LAWB* }\end{array}$ & $\begin{array}{l}>10 \% \\
\text { LAWC }\end{array}$ \\
\hline $\begin{array}{l}2.2 .2 .3 \\
2.2 .2 .4 \\
2.2 .2 .5\end{array}$ & $\begin{array}{c}\text { Cylindrical package is } 1.22 \mathrm{~m} \text { in } \\
\text { diameter and } 2.3 \mathrm{~m} \text { high; mass shall not } \\
\text { exceed } 10,000 \mathrm{~kg} \text {. } \\
\text { Void shall not exceed } 10 \% \text {. }\end{array}$ & \multicolumn{3}{|c|}{ Density of Glass $<3.7$} \\
\hline \begin{tabular}{|l|}
2.2 .2 .6 .1 \\
$2.2 \cdot 2 \cdot 6.2$
\end{tabular} & Chemical composition of Glass & \multicolumn{3}{|c|}{$\begin{array}{c}\text { Report all components greater than } 0.5 \% \\
\text { and all regulated elements }\end{array}$} \\
\hline 2.2.2.6.3 & Crystalline Phase Identification & \multicolumn{3}{|c|}{$\begin{array}{c}\text { Report amount of crystals and provide } \\
\text { TTT }\end{array}$} \\
\hline $\begin{array}{l}\text { LAW SPECIFICATION IID } \\
\text { SP-W375LV-M0001 and [43] }\end{array}$ & Liquidus & \multicolumn{3}{|c|}{$<950^{\circ} \mathrm{C}$} \\
\hline ILAW WFQ & $\begin{array}{c}\text { Subjected to LAW Centerline Canister } \\
\text { Cooling }\end{array}$ & \multicolumn{3}{|c|}{ Report amount of crystals } \\
\hline 2.2.2.17.1 & $\begin{array}{l}\text { Leachability Index per } \\
\text { ANSI/ANS-16.1 }\end{array}$ & \multicolumn{3}{|c|}{$>6.0$ after 90 days } \\
\hline 2.2.2.17.2 & $\begin{array}{l}\text { PCT per ASTM C1285-98. } \\
\left.\text { (7 days at } 90^{\circ} \mathrm{C}\right) \\
\mathrm{B}\left(\mathrm{g} / \mathrm{m}^{2}\right) \\
\mathrm{Na}\left(\mathrm{g} / \mathrm{m}^{2}\right) \\
\mathrm{Si}\left(\mathrm{g} / \mathrm{m}^{2}\right) \\
\end{array}$ & \multicolumn{3}{|c|}{$<2.0 \mathrm{~g} / \mathrm{m}^{2}$} \\
\hline 2.2.2.17.3 & $\begin{array}{l}\mathrm{VHT} \text { at } 200^{\circ} \mathrm{C}\left(\mathrm{g} / \mathrm{m}^{2} / \mathrm{day}\right) \\
\text { for seven days }\end{array}$ & \multicolumn{3}{|c|}{$<50 \mathrm{~g} / \mathrm{m}^{2} /$ day } \\
\hline 2.2 .2 .18 & Compressive strength & \multicolumn{3}{|c|}{ > $3.45 \mathrm{MPa}$} \\
\hline 2.2.2.19 & $\begin{array}{l}\text { Compressive strength after Thermal, } \\
\text { Radiation, Biodegradation and } \\
\text { Immersion per ANSI/ANS } 16.1 \\
\end{array}$ & \multicolumn{3}{|c|}{$>75 \%$ of initial strength } \\
\hline 2.2.2.20 & $\begin{array}{c}\text { ILAW package acceptable for land } \\
\text { disposal under State of Washington } \\
\text { WAC 173-303 and RCRA LDR } \\
\text { Biotoxicity } \\
\end{array}$ & \multicolumn{3}{|c|}{ Meets TCLP limits } \\
\hline $\begin{array}{l}\text { LAW SPECIFICATION IID SP- } \\
\text { W375LV-M0001** and [43] }\end{array}$ & Viscosity Data (poise) at $1100^{\circ} \mathrm{C}$ & \multicolumn{3}{|c|}{10 to $150 \mathrm{P}$} \\
\hline $\begin{array}{l}\text { LAW SPECIFICATION IID SP- } \\
\text { W375LV-M0001** and [43] }\end{array}$ & Conductivity $(\mathrm{S} / \mathrm{cm})$ at $1100^{\circ} \mathrm{C}$ & \multicolumn{3}{|c|}{0.2 to $0.7 \mathrm{~S} / \mathrm{cm}$} \\
\hline ILAW WFQ & $\begin{array}{c}\mathrm{T}_{\mathrm{G}} \text { (onset) } \\
\text { Package Storage }\end{array}$ & \multicolumn{3}{|c|}{ Required for canister cooling modeling } \\
\hline
\end{tabular}

* For LAW AZ-102 this requirement was changed to "reduced waste loading as necessary to avoid excessive K-3 corrosion and other negative effects caused by the high sulfate to sodium ratio" via WTP Test Exception 24590-LAW-TEF-RT-02-002 dated 10/21/02.

** Since the present work was completed, this reference was superceded by: Clark, K. 2003. Engineering Specification for Low Activity Waste Melters, 24590-LAW-3PS-AE00-T0001, River Protection Project Waste Treatment Plant, Richland, Washington, 99352,which revises the lower conductivity limit to 0.1 $\mathrm{S} / \mathrm{cm}$. 
Table 2.1. Compositions (Molarity) of the Nine LAW Tank Wastes as Given in TFCOUP Rev. 2.

\begin{tabular}{|c|c|c|c|c|c|c|c|c|c|}
\hline $\begin{array}{l}\text { TFCOUP Rev.2 } \\
\text { Batch \# }\end{array}$ & LAW-1 & LAW-2a & LAW-2b & LAW-3 & LAW-5 & LAW-7 & LAW-8 & LAW-14 & LAW-15 \\
\hline Constituents & AP-101 Sup & AZ-101 Sup & AZ-102 Sup & AN-102 Sup & AN-104 DS & AN-107 Sup & AN-105 Sup & AW-101 Sup & AW-101 DS \\
\hline $\mathrm{Al}$ & 0.366 & 0.648 & 0.173 & 0.465 & 0.784 & 0.236 & 0.881 & 0.682 & 0.699 \\
\hline $\mathrm{Ba}$ & 2.7E-06 & $3.1 \mathrm{E}-05$ & $1.2 \mathrm{E}-10$ & $1.8 \mathrm{E}-04$ & $8.2 \mathrm{E}-05$ & 0 & $1.3 \mathrm{E}-04$ & $1.5 \mathrm{E}-05$ & $0.0 \mathrm{E}+00$ \\
\hline $\mathrm{Ca}$ & 0.001 & 0.000 & 0.000 & 0.013 & 0.001 & 0.013 & 0.001 & 0.001 & 0.001 \\
\hline $\mathrm{Cd}$ & 9.5E-06 & $6.7 \mathrm{E}-05$ & $2.6 \mathrm{E}-10$ & 4.7E-04 & $1.0 \mathrm{E}-05$ & 0 & $3.4 \mathrm{E}-05$ & $1.4 \mathrm{E}-06$ & 0 \\
\hline Cr (Total) & 0.002 & 0.024 & 0.060 & 0.007 & 0.004 & 0.005 & 0.003 & 0.003 & 0.002 \\
\hline $\mathrm{Fe}$ & 0.000 & 0.000 & 0.001 & 0.003 & 0.000 & 0.046 & 0.000 & 0.000 & 0.000 \\
\hline $\mathrm{K}$ & 0.469 & 0.192 & 0.247 & 0.080 & 0.084 & 0.039 & 0.100 & 0.446 & 0.569 \\
\hline $\mathrm{La}$ & 0 & $3.6 \mathrm{E}-10$ & $6.1 \mathrm{E}-05$ & 0 & 8.3E-06 & $2.3 \mathrm{E}-04$ & $4.3 \mathrm{E}-13$ & $6.7 \mathrm{E}-12$ & $8.1 \mathrm{E}-12$ \\
\hline $\mathrm{Na}$ & 6.506 & 7.705 & 7.373 & 9.000 & 6.897 & 8.452 & 6.934 & 6.994 & 6.998 \\
\hline $\mathrm{Ni}$ & $1.1 \mathrm{E}-04$ & $1.6 \mathrm{E}-06$ & $1.1 \mathrm{E}-04$ & 5.6E-03 & 8.5E-05 & 7.7E-03 & 3.7E-04 & $1.4 \mathrm{E}-04$ & 1.5E-04 \\
\hline $\mathrm{Pb}$ & $1.6 \mathrm{E}-05$ & 0 & 0 & 7.8E-04 & $1.3 \mathrm{E}-04$ & $1.8 \mathrm{E}-03$ & $1.8 \mathrm{E}-04$ & $1.9 \mathrm{E}-04$ & 2.2E-04 \\
\hline U (all U isotopes) & 4.9E-07 & 4.5E-08 & 6.7E-07 & 4.3E-07 & $1.7 \mathrm{E}-08$ & 0 & $3.0 \mathrm{E}-08$ & $3.5 \mathrm{E}-07$ & 5.2E-07 \\
\hline $\mathrm{Cl}$ & 0.038 & 0.009 & 0.001 & 0.087 & 0.143 & 0.048 & 0.173 & 0.118 & 0.097 \\
\hline $\mathrm{F}$ & 0.124 & 0.158 & 0.171 & 0.079 & 0.020 & 0.163 & 0.023 & 0.057 & 0.055 \\
\hline $\mathrm{PO}_{4}$ & 0.011 & 0.025 & 0.004 & 0.041 & 0.028 & 0.035 & 0.015 & 0.015 & 0.008 \\
\hline $\mathrm{SO}_{4}$ & 0.037 & 0.302 & 0.584 & 0.132 & 0.067 & 0.081 & 0.030 & 0.024 & 0.015 \\
\hline TIC & 0.238 & 0.909 & 1.798 & 0.924 & 0.542 & 1.139 & 0.280 & 0.354 & 0.215 \\
\hline $\mathrm{CO}_{3}^{-2}$ & 0.238 & 0.909 & 1.798 & 0.925 & 0.525 & 1.138 & 0.280 & 0.354 & 0.215 \\
\hline TOC & 0.268 & 0.191 & 0.410 & 1.778 & 0.162 & 2.923 & 0.390 & 0.206 & 0.169 \\
\hline $\mathrm{NH}_{3}$ & 0.000 & 0.041 & 0.077 & 0.070 & 0.129 & 0.041 & 0.112 & 0.419 & 0.543 \\
\hline $\mathrm{NO}_{2}$ & 0.897 & 2.280 & 2.047 & 1.441 & 1.640 & 1.208 & 1.663 & 1.487 & 1.419 \\
\hline $\mathrm{NO}_{3}$ & 2.171 & 1.961 & 1.163 & 2.921 & 1.900 & 3.210 & 1.775 & 1.852 & 1.813 \\
\hline $\mathrm{OH}$ & 4.173 & 3.188 & 0.436 & 3.211 & 5.272 & 11.140 & 5.331 & 5.251 & 5.840 \\
\hline
\end{tabular}


Table 3.1. Waste Contribution to the Glass Composition for Tank AN-105 Supernatant (Batch 8) Per TFCOUP Rev. 2 and Target Compositions of High-Sulfate LAWA44 Glasses.

\begin{tabular}{|c|c|c|c|c|c|c|c|c|}
\hline $\begin{array}{l}\text { Glass } \\
\text { Oxides }\end{array}$ & $\begin{array}{c}\text { Composition } \\
\text { of LAW } \\
\text { AN-105 } \\
\text { (Batch 8) }\end{array}$ & $\begin{array}{c}\text { LAW AN- } \\
105 \text { Waste } \\
\text { Contribution } \\
\text { to Glass }\end{array}$ & $\begin{array}{l}\text { LAW AN- } \\
105 \text { Waste } \\
\text { Contribution } \\
\text { (including } \\
\text { pretreatment) }\end{array}$ & $\begin{array}{c}\text { Glass Forn } \\
\mathrm{L}\end{array}$ & $\begin{array}{l}\text { ditives for } \\
4\end{array}$ & LAWA44S1 & LAWA44S2 & LAWA44SX \\
\hline Loading & $100 \%$ & $24.96 \%$ & $25.46 \%$ & $100.00 \%$ & $74.54 \%$ & $25.46 \%$ & $25.46 \%$ & $25.46 \%$ \\
\hline $\mathrm{Al}_{2} \mathrm{O}_{3}$ & 16.33 & 4.07 & 4.07 & 2.86 & 2.13 & 6.21 & 6.21 & 6.21 \\
\hline $\mathrm{CaO}$ & 0.03 & 0.01 & 0.01 & 2.67 & 1.99 & 2.00 & 2.00 & 2.00 \\
\hline $\mathrm{Cr}_{2} \mathrm{O}_{3}$ & 0.09 & 0.02 & 0.02 & - & - & 0.02 & 0.02 & 0.02 \\
\hline $\mathrm{Fe}_{2} \mathrm{O}_{3}$ & 0.01 & 0.00 & 0.00 & 9.36 & 6.98 & 6.98 & 6.98 & 6.98 \\
\hline $\mathrm{K}_{2} \mathrm{O}$ & 1.72 & 0.43 & 0.43 & - & - & 0.43 & 0.43 & 0.43 \\
\hline $\mathrm{MgO}$ & - & - & - & 2.68 & 2.00 & 2.00 & 2.00 & 2.00 \\
\hline $\mathrm{Na}_{2} \mathrm{O}$ & 78.13 & 19.50 & 20.00 & - & - & 20.00 & 20.00 & $21.50 *$ \\
\hline $\mathrm{SiO}_{2}$ & - & - & - & 59.80 & 44.58 & 44.58 & 44.58 & 44.58 \\
\hline $\mathrm{TiO}_{2}$ & - & - & - & 2.68 & 2.00 & 2.00 & 2.00 & 2.00 \\
\hline $\mathrm{ZnO}$ & - & - & - & 3.98 & 2.97 & 2.97 & 2.97 & 2.97 \\
\hline $\mathrm{ZrO}_{2}$ & - & - & - & 4.02 & 3.00 & 3.00 & 3.00 & 3.00 \\
\hline $\mathrm{Cl}$ & 2.23 & 0.56 & 0.56 & - & - & 0.56 & 0.56 & 0.56 \\
\hline $\mathrm{F}$ & 0.16 & 0.04 & 0.04 & - & - & 0.04 & 0.04 & 0.04 \\
\hline $\mathrm{P}_{2} \mathrm{O}_{5}$ & 0.39 & 0.10 & 0.10 & - & - & 0.10 & 0.10 & 0.10 \\
\hline $\mathrm{SO}_{3}$ & 0.88 & 0.22 & 0.22 & - & - & 1.48 & 2.50 & 2.50 \\
\hline $\mathrm{Re}_{2} \mathrm{O}_{7}$ & & 0.00 & 0.00 & - & - & 0.10 & 0.10 & 0.10 \\
\hline SUM & 100.00 & 24.96 & 25.46 & 100.00 & 74.56 & 101.35 & 102.37 & 103.87 \\
\hline
\end{tabular}

* Extra sodium added to compensate for partitioning into sulfate phase. 
Table 3.2. Composition of Glasses for LAW Tank AN-105 Supernatant (Batch 8) per TFCOUP Rev. 2.

\begin{tabular}{|c|c|c|c|c|c|c|c|c|c|c|c|c|}
\hline $\begin{array}{l}\text { Glass } \\
\text { Oxides }\end{array}$ & $\begin{array}{c}\text { LAW } \\
\text { AN-105 } \\
\text { Pretreated } \\
\text { wt\% }\end{array}$ & $\begin{array}{l}\text { Delta } \\
\text { to } \\
\text { LAW } \\
\text { A44 }\end{array}$ & \multicolumn{2}{|c|}{$\begin{array}{l}\text { Glass Former } \\
\text { Additives for } \\
\text { LAWA108 }\end{array}$} & \multirow{2}{*}{$\begin{array}{c}\begin{array}{c}\text { LAWA108 } \\
\text { S1 } \\
\text { (target) }\end{array} \\
25.46 \%\end{array}$} & \multirow{2}{*}{$\begin{array}{c}\text { LAWA108 } \\
\begin{array}{c}\text { S2 } \\
\text { (target) }\end{array} \\
25.46 \%\end{array}$} & \multirow{2}{*}{$\begin{array}{c}\text { LAWA108 } \\
\text { S0 } \\
\text { (target) } \\
25.46 \%\end{array}$} & \multirow{2}{*}{$\begin{array}{c}\text { Delta to } \\
\text { LAW } \\
\text { A44 } \\
-\end{array}$} & \multicolumn{2}{|c|}{$\begin{array}{l}\text { Glass Former } \\
\text { Additives for } \\
\text { LAWA109 }\end{array}$} & \multirow{2}{*}{$\begin{array}{c}\text { LAWA109 } \\
\begin{array}{c}\text { S1 } \\
\text { (target) }\end{array} \\
25.46 \%\end{array}$} & \multirow{2}{*}{$\begin{array}{c}\begin{array}{c}\text { LAWA109 } \\
\text { S2 } \\
\text { (target) }\end{array} \\
25.46 \%\end{array}$} \\
\hline Loading & $25.46 \%$ & - & $100.00 \%$ & $74.54 \%$ & & & & & $\begin{array}{c}100.00 \\
\%\end{array}$ & $74.54 \%$ & & \\
\hline $\mathrm{Al}_{2} \mathrm{O}_{3}$ & 4.07 & - & 2.86 & 2.13 & 6.21 & 6.21 & 6.21 & - & 2.86 & 2.13 & 6.21 & 6.21 \\
\hline $\mathrm{B}_{2} \mathrm{O}_{3}$ & - & 3.49 & 16.63 & 12.40 & 12.40 & 12.40 & 12.40 & 1.75 & 14.29 & 10.65 & 10.65 & 10.65 \\
\hline $\mathrm{CaO}$ & 0.01 & - & 2.67 & 1.99 & 2.00 & 2.00 & 2.00 & - & 2.67 & 1.99 & 2.00 & 2.00 \\
\hline $\mathrm{Cr}_{2} \mathrm{O}_{3}$ & 0.02 & - & & & 0.02 & 0.02 & 0.02 & - & - & - & 0.02 & 0.02 \\
\hline $\mathrm{Fe}_{2} \mathrm{O}_{3}$ & 0.00 & -6.98 & 0.00 & 0.00 & 0.00 & 0.00 & 0.00 & -3.49 & 4.68 & 3.49 & 3.49 & 3.49 \\
\hline $\mathrm{K}_{2} \mathrm{O}$ & 0.43 & - & & & 0.43 & 0.43 & 0.43 & - & - & - & 0.43 & 0.43 \\
\hline $\mathrm{MgO}$ & - & - & 2.68 & 2.00 & 2.00 & 2.00 & 2.00 & - & 2.68 & 2.00 & 2.00 & 2.00 \\
\hline $\mathrm{Na}_{2} \mathrm{O}$ & 20.00 & - & & & 20.00 & 20.00 & 20.00 & - & - & - & 20.00 & 20.00 \\
\hline $\mathrm{SiO}_{2}$ & - & 3.49 & 64.48 & 48.07 & 48.07 & 48.07 & 48.07 & 1.75 & 62.14 & 46.32 & 46.32 & 46.32 \\
\hline $\mathrm{TiO}_{2}$ & - & - & 2.68 & 2.00 & 2.00 & 2.00 & 2.00 & - & 2.68 & 2.00 & 2.00 & 2.00 \\
\hline $\mathrm{ZnO}$ & - & - & 3.98 & 2.97 & 2.97 & 2.97 & 2.97 & - & 3.98 & 2.97 & 2.97 & 2.97 \\
\hline $\mathrm{ZrO}_{2}$ & - & - & 4.02 & 3.00 & 3.00 & 3.00 & 3.00 & - & 4.02 & 3.00 & 3.00 & 3.00 \\
\hline $\mathrm{Cl}$ & 0.56 & - & - & - & 0.56 & 0.56 & 0.56 & - & - & - & 0.56 & 0.56 \\
\hline $\mathrm{F}$ & 0.04 & - & - & - & 0.04 & 0.04 & 0.04 & - & - & - & 0.04 & 0.04 \\
\hline $\mathrm{P}_{2} \mathrm{O}_{5}$ & 0.10 & - & - & - & 0.10 & 0.10 & 0.10 & - & - & - & 0.10 & 0.10 \\
\hline $\mathrm{SO}_{3}$ & 0.22 & - & - & - & 1.48 & 2.50 & 0.00 & - & - & - & 1.48 & 2.50 \\
\hline $\mathrm{Re}_{2} \mathrm{O}_{7}$ & 0.00 & - & - & - & 0.1 & 0.1 & 0.1 & - & - & - & 0.1 & 0.1 \\
\hline SUM & 25.46 & - & 100.00 & 74.54 & 101.35 & 102.37 & 99.87 & - & 100.00 & 74.54 & 101.35 & 102.37 \\
\hline
\end{tabular}


Table 3.2. Composition of Glasses for Tank AN-105 Supernatant (Batch 8) per TFCOUP Rev. 2 (cont'd).

\begin{tabular}{|c|c|c|c|c|c|c|c|c|c|c|c|}
\hline $\begin{array}{l}\text { Glass } \\
\text { Oxides }\end{array}$ & $\begin{array}{c}\text { LAW } \\
\text { AN-105 } \\
\text { Pretreated } \\
\text { wt\% }\end{array}$ & $\begin{array}{l}\text { Delta } \\
\text { to } \\
\text { LAW } \\
\text { A44 }\end{array}$ & \multicolumn{2}{|c|}{$\begin{array}{l}\text { Glass Former } \\
\text { Additives for } \\
\text { LAWA110 }\end{array}$} & \multirow{2}{*}{$\begin{array}{c}\text { LAWA110 } \\
\begin{array}{c}\text { S1 } \\
\text { (target) }\end{array} \\
25.46 \%\end{array}$} & \multirow{2}{*}{$\begin{array}{c}\text { LAWA110 } \\
\begin{array}{c}\text { S2 } \\
\text { (target) }\end{array} \\
25.46 \%\end{array}$} & \multirow{2}{*}{$\begin{array}{c}\text { Delta } \\
\text { to } \\
\text { LAW } \\
\text { A44 } \\
-\end{array}$} & \multicolumn{2}{|c|}{$\begin{array}{c}\text { Glass Former } \\
\text { Additives for } \\
\text { LAWA111 }\end{array}$} & \multirow{2}{*}{$\begin{array}{c}\text { LAWA111 } \\
\begin{array}{c}\text { S1 } \\
\text { (target) }\end{array} \\
25.46 \%\end{array}$} & \multirow{2}{*}{$\begin{array}{c}\text { LAWA111 } \\
\begin{array}{c}\text { S2 } \\
\text { (target) }\end{array} \\
25.46 \% \\
\end{array}$} \\
\hline Loading & $25.46 \%$ & - & $100.00 \%$ & $74.54 \%$ & & & & $100.00 \%$ & $74.54 \%$ & & \\
\hline $\mathrm{Al}_{2} \mathrm{O}_{3}$ & 4.07 & 1.16 & 4.42 & 3.30 & 7.37 & 7.37 & - & 2.86 & 2.13 & 6.21 & 6.21 \\
\hline $\mathrm{B}_{2} \mathrm{O}_{3}$ & - & 1.16 & 13.51 & 10.07 & 10.07 & 10.07 & - & 11.95 & 8.91 & 8.91 & 8.91 \\
\hline $\mathrm{CaO}$ & 0.01 & $1.16-$ & 4.23 & 3.15 & 3.16 & 3.16 & 6.98 & 12.03 & 8.97 & 8.98 & 8.98 \\
\hline $\mathrm{Cr}_{2} \mathrm{O}_{3}$ & 0.02 & - & & & 0.02 & 0.02 & - & - & - & 0.02 & 0.02 \\
\hline $\mathrm{Fe}_{2} \mathrm{O}_{3}$ & 0.00 & -3.49 & 4.68 & 3.49 & 3.49 & 3.49 & -6.98 & 0.00 & 0.00 & 0.00 & 0.00 \\
\hline $\mathrm{K}_{2} \mathrm{O}$ & 0.43 & - & & & 0.43 & 0.43 & - & - & - & 0.43 & 0.43 \\
\hline $\mathrm{MgO}$ & - & - & 2.68 & 2.00 & 2.00 & 2.00 & - & 2.68 & 2.00 & 2.00 & 2.00 \\
\hline $\mathrm{Na}_{2} \mathrm{O}$ & 20.00 & - & & & 20.00 & 20.00 & - & - & - & 20.00 & 20.00 \\
\hline $\mathrm{SiO}_{2}$ & - & 0.00 & 59.80 & 44.58 & 48.48 & 48.48 & 0.00 & 59.80 & 44.58 & 44.58 & 44.58 \\
\hline $\mathrm{TiO}_{2}$ & - & - & 2.68 & 2.00 & 2.00 & 2.00 & - & 2.68 & 2.00 & 2.00 & 2.00 \\
\hline $\mathrm{ZnO}$ & - & - & 3.98 & 2.97 & 2.97 & 2.97 & - & 3.98 & 2.97 & 2.97 & 2.97 \\
\hline $\mathrm{ZrO}_{2}$ & - & - & 4.02 & 3.00 & 3.00 & 3.00 & - & 4.02 & 3.00 & 3.00 & 3.00 \\
\hline $\mathrm{Cl}$ & 0.56 & - & - & - & 0.56 & 0.56 & - & - & - & 0.56 & 0.56 \\
\hline $\mathrm{F}$ & 0.04 & - & - & - & 0.04 & 0.04 & - & - & - & 0.04 & 0.04 \\
\hline $\mathrm{P}_{2} \mathrm{O}_{5}$ & 0.10 & - & - & - & 0.10 & 0.10 & - & - & - & 0.10 & 0.10 \\
\hline $\mathrm{SO}_{3}$ & 0.22 & - & - & - & 1.48 & 2.50 & - & - & - & 1.48 & 2.50 \\
\hline $\mathrm{Re}_{2} \mathrm{O}_{7}$ & 0.00 & - & - & - & 0.1 & 0.1 & - & - & - & 0.1 & 0.1 \\
\hline SUM & 25.46 & - & 100.00 & 74.54 & 101.35 & 102.37 & - & 100.00 & 74.54 & 101.35 & 102.37 \\
\hline
\end{tabular}


Table 3.2. Composition of Glasses for Tank AN-105 Supernatant (Batch 8) per TFCOUP Rev. 2 (cont'd).

\begin{tabular}{|c|c|c|c|c|c|c|}
\hline $\begin{array}{l}\text { Glass } \\
\text { Oxides }\end{array}$ & \multirow[t]{2}{*}{$\begin{array}{l}\text { Delta to } \\
\text { LAWA44 }\end{array}$} & \multirow{2}{*}{$\begin{array}{c}\text { LAW AN-105 } \\
\begin{array}{c}\text { Pretreated } \\
\text { wt\% }\end{array} \\
25.46 \% \\
\end{array}$} & \multicolumn{2}{|c|}{$\begin{array}{l}\text { Glass Former Additives for } \\
\text { LAWA133 }\end{array}$} & \multirow{2}{*}{$\begin{array}{c}\begin{array}{c}\text { LAWA133 } \\
\text { (target) }\end{array} \\
25.46 \% \\
\end{array}$} & \multirow{2}{*}{$\begin{array}{c}\text { LAWA133S2 } \\
\text { (target) } \\
25.46 \%\end{array}$} \\
\hline Loading & & & $100.00 \%$ & $74.54 \%$ & & \\
\hline $\mathrm{Al}_{2} \mathrm{O}_{3}$ & - & 4.07 & 2.86 & 2.13 & 6.21 & 6.21 \\
\hline $\mathrm{B}_{2} \mathrm{O}_{3}$ & - & - & 11.95 & 8.91 & 8.91 & 8.91 \\
\hline $\mathrm{CaO}$ & 3.49 & 0.01 & 7.35 & 5.48 & 5.49 & 5.49 \\
\hline $\mathrm{Cr}_{2} \mathrm{O}_{3}$ & - & 0.02 & - & - & 0.02 & 0.02 \\
\hline $\mathrm{Fe}_{2} \mathrm{O}_{3}$ & -3.49 & 0.00 & 4.68 & 3.49 & 3.49 & 3.49 \\
\hline $\mathrm{K}_{2} \mathrm{O}$ & - & 0.43 & - & - & 0.43 & 0.43 \\
\hline $\mathrm{MgO}$ & - & - & 2.68 & 2.00 & 2.00 & 2.00 \\
\hline $\mathrm{Na}_{2} \mathrm{O}$ & - & 20.00 & - & - & 20.00 & 20.00 \\
\hline $\mathrm{SiO}_{2}$ & - & - & 59.80 & 44.58 & 44.58 & 44.58 \\
\hline $\mathrm{TiO}_{2}$ & - & - & 2.68 & 2.00 & 2.00 & 2.00 \\
\hline $\mathrm{ZnO}$ & - & - & 3.98 & 2.97 & 2.97 & 2.97 \\
\hline $\mathrm{ZrO}_{2}$ & - & - & 4.02 & 3.00 & 3.00 & 3.00 \\
\hline $\mathrm{Cl}$ & - & 0.56 & - & - & 0.56 & 0.56 \\
\hline $\mathrm{F}$ & - & 0.04 & - & - & 0.04 & 0.04 \\
\hline $\mathrm{P}_{2} \mathrm{O}_{5}$ & - & 0.10 & - & - & 0.10 & 0.10 \\
\hline $\mathrm{SO}_{3}$ & - & 0.22 & - & - & 0.22 & 2.50 \\
\hline $\mathrm{Re}_{2} \mathrm{O}_{7}$ & - & 0.00 & - & - & 0.10 & 0.10 \\
\hline SUM & - & 25.46 & 100.00 & 74.54 & 100.10 & 102.38 \\
\hline
\end{tabular}


Table 3.3. Waste Contribution to the Glass Composition for Tank AP-101 Supernatant Per TFCOUP Rev. 2.

\begin{tabular}{|c|c|c|c|c|c|c|c|c|c|}
\hline $\begin{array}{l}\text { Glass } \\
\text { Oxides }\end{array}$ & $\begin{array}{l}\text { Composition } \\
\text { of LAW } \\
\text { AP-101 per } \\
\text { TFCOUP } \\
\text { Rev.2 }\end{array}$ & $\begin{array}{c}\text { LAW AP- } \\
101 \\
\text { Contribution } \\
\text { to Glass } \\
\text { wt\% }\end{array}$ & $\begin{array}{c}\text { Contribution } \\
\text { from } \\
\text { pretreated } \\
\text { LAW } \\
\text { AP-101 }\end{array}$ & $\begin{array}{r}\text { Glass } \\
\text { Addi } \\
\text { LA }\end{array}$ & $\begin{array}{l}\text { ormer } \\
\text { es for } \\
488\end{array}$ & $\begin{array}{c}\text { LAWA88CC } \\
\text { (target) }\end{array}$ & $\begin{array}{l}\text { LAWA88S1 } \\
\text { (target) }\end{array}$ & $\begin{array}{l}\text { LAWA88S2 } \\
\text { (target) }\end{array}$ & $\begin{array}{l}\text { LAWA88SX } \\
\text { (target) }\end{array}$ \\
\hline Loading & $100 \%$ & $24.18 \%$ & $24.78 \%$ & $100 \%$ & $75.22 \%$ & \multicolumn{4}{|c|}{ Waste contribution $=24.18 \%$} \\
\hline $\mathrm{Al}_{2} \mathrm{O}_{3}$ & 7.47 & 1.81 & 1.81 & 5.71 & 4.29 & 6.10 & 6.10 & 6.10 & 6.10 \\
\hline $\mathrm{B}_{2} \mathrm{O} 3$ & - & - & - & 13.07 & 9.83 & 9.83 & 9.83 & 9.83 & 9.83 \\
\hline $\mathrm{CaO}$ & 0.02 & 0.01 & 0.01 & 2.65 & 1.99 & 2.00 & 2.00 & 2.00 & 2.00 \\
\hline $\mathrm{Cr}_{2} \mathrm{O}_{3}$ & 0.07 & 0.02 & 0.02 & - & - & 0.02 & 0.02 & 0.02 & 0.02 \\
\hline $\mathrm{Fe}_{2} \mathrm{O}_{3}$ & 0.00 & 0.00 & 0.00 & 7.38 & 5.55 & 5.55 & 5.55 & 5.55 & 5.55 \\
\hline $\mathrm{K}_{2} \mathrm{O}$ & 8.83 & 2.14 & 2.14 & - & - & 2.14 & 2.14 & 2.14 & 2.14 \\
\hline $\mathrm{MgO}$ & - & - & - & 1.97 & 1.48 & 1.48 & 1.48 & 1.48 & 1.48 \\
\hline $\mathrm{Na}_{2} \mathrm{O}$ & 80.65 & 19.50 & 20.00 & - & - & 20.00 & 20.00 & 20.00 & 21.30 \\
\hline $\mathrm{SiO}_{2}$ & 0.00 & 0.00 & 0.00 & 58.67 & 44.13 & 44.13 & 44.13 & 44.13 & 44.13 \\
\hline $\mathrm{TiO}_{2}$ & 0.00 & 0.00 & 0.00 & 2.66 & 2.00 & 2.00 & 2.00 & 2.00 & 2.00 \\
\hline $\mathrm{ZnO}$ & - & - & - & 3.93 & 2.96 & 2.96 & 2.96 & 2.96 & 2.96 \\
\hline $\mathrm{ZrO}_{2}$ & - & - & - & 3.98 & 3.00 & 3.00 & 3.00 & 3.00 & 3.00 \\
\hline $\mathrm{Cl}$ & 0.54 & 0.13 & 0.13 & - & - & 0.13 & 0.13 & 0.13 & 0.13 \\
\hline $\mathrm{F}$ & 0.94 & 0.23 & 0.23 & - & - & 0.23 & 0.23 & 0.23 & 0.23 \\
\hline $\mathrm{P}_{2} \mathrm{O}_{5}$ & 0.30 & 0.07 & 0.07 & - & - & 0.07 & 0.07 & 0.07 & 0.07 \\
\hline $\mathrm{SO}_{3}$ & 1.17 & 0.28 & 0.28 & - & - & 0.28 & 1.40 & 2.50 & 2.50 \\
\hline $\mathrm{Re}_{2} \mathrm{O}_{7}$ spike & - & - & 0.10 & - & - & 0.10 & 0.10 & 0.10 & 0.10 \\
\hline Sum & 100.00 & 24.18 & 24.68 & 100.00 & 75.22 & 100.00 & 101.19 & 102.29 & 103.59 \\
\hline
\end{tabular}


Table 3.4. LAW AP-101 Waste Composition as Reported in PNNL-13354 [8] and Waste Contribution to Glasses.

\begin{tabular}{|c|c|c|c|c|c|c|c|c|c|c|}
\hline \multirow[t]{2}{*}{$\begin{array}{c}\text { Envelope } \\
\text { Constituents }\end{array}$} & \multicolumn{2}{|c|}{$\begin{array}{l}\text { LAW AP-101 as } \\
\text { Given in [8] } \\
\text { At } 5.62 \mathrm{M} \mathrm{Na}\end{array}$} & \multirow{2}{*}{$\begin{array}{r}\text { Glass } \\
\text { Oxides } \\
\text { Loading }\end{array}$} & \multirow{2}{*}{$\begin{array}{c}\begin{array}{c}\text { Conversion } \\
\text { to wt\% }\end{array} \\
\text { "Oxides" }\end{array}$} & \multicolumn{3}{|c|}{$\begin{array}{c}\text { LAW AP-101 } \\
\text { Waste Contribution to Glass (wt\%) } \\
\text { at } 3 \text { Waste Loading Tested }\end{array}$} & \multicolumn{3}{|c|}{$\begin{array}{l}\text { LAW AP-101 } \\
\text { Waste Contribution to Glass (wt\%) } \\
\text { Including Pretreatment }\end{array}$} \\
\hline & Moles/L & $\mathrm{mg} / \mathrm{L}$ & & & $24.22 \%$ & $23.28 \%$ & $22.34 \%$ & $24.68 \%$ & $23.72 \%$ & $22.76 \%$ \\
\hline $\mathrm{Al}$ & 0.257 & 6940 & $\mathrm{Al}_{2} \mathrm{O}_{3}$ & 5.49 & 1.35 & 1.30 & 1.25 & 1.35 & 1.30 & 1.25 \\
\hline $\mathrm{B}$ & 0.001 & 15 & $\mathrm{~B}_{2} \mathrm{O}_{3}$ & 0.02 & 0.01 & 0.01 & 0.01 & 0.01 & 0.01 & 0.01 \\
\hline $\mathrm{Ca}$ & 0.000 & 7.7 & $\mathrm{CaO}$ & 0.00 & - & - & - & - & - & - \\
\hline $\mathrm{Cr}$ & 0.003 & 143 & $\mathrm{Cr}_{2} \mathrm{O}_{3}$ & 0.09 & 0.02 & 0.02 & 0.02 & 0.02 & 0.02 & 0.02 \\
\hline $\mathrm{Fe}$ & 0.000 & 2.5 & $\mathrm{Fe}_{2} \mathrm{O}_{3}$ & 0.00 & - & - & - & - & - & - \\
\hline $\mathrm{K}$ & 0.798 & 31200 & $\mathrm{~K}_{2} \mathrm{O}$ & 15.72 & 3.88 & 3.73 & 3.58 & 3.88 & 3.73 & 3.58 \\
\hline $\mathrm{Na}$ & 5.624 & 129300 & $\mathrm{Na}_{2} \mathrm{O}$ & 74.79 & 18.00 & 17.30 & 16.60 & 18.46 & 17.74 & 17.03 \\
\hline $\mathrm{Si}$ & 0.005 & 137 & $\mathrm{SiO}_{2}$ & 0.12 & 0.03 & 0.03 & 0.03 & 0.03 & 0.03 & 0.03 \\
\hline $\mathrm{Zn}$ & 0.000 & 5.6 & $\mathrm{ZnO}$ & 0.00 & - & - & - & - & - & - \\
\hline $\mathrm{Zr}$ & 0.000 & 1.4 & $\mathrm{ZrO}_{2}$ & 0.00 & - & - & - & - & - & - \\
\hline $\mathrm{Cl}$ & 0.056 & 1980 & $\mathrm{Cl}$ & 0.83 & 0.20 & 0.20 & 0.19 & 0.20 & 0.20 & 0.19 \\
\hline $\mathrm{F}$ & 0.153 & 2900 & $\mathrm{~F}$ & 1.21 & 0.30 & 0.29 & 0.28 & 0.30 & 0.29 & 0.28 \\
\hline $\mathrm{PO}_{4}$ & 0.011 & 1020 & $\mathrm{P}_{2} \mathrm{O}_{5}$ & 0.32 & 0.08 & 0.08 & 0.07 & 0.08 & 0.08 & 0.07 \\
\hline $\mathrm{SO}_{4}$ & 0.042 & 4030 & $\mathrm{SO}_{3}$ & 1.41 & 0.35 & 0.33 & 0.32 & 0.35 & 0.33 & 0.32 \\
\hline $\mathrm{NO}_{2}$ & 0.911 & 41900 & - & - & - & - & - & - & - & - \\
\hline $\mathrm{NO}_{3}$ & 2.129 & 132000 & - & - & - & - & - & - & - & - \\
\hline $\mathrm{CO}_{3}$ & 0.538 & 6460 & - & - & - & - & - & - & - & - \\
\hline $\mathrm{OH}$ & 2.429 & 41300 & - & - & - & - & - & - & - & - \\
\hline Org. Carbon & 0.163 & 1960 & - & - & - & - & - & - & - & - \\
\hline SUM & - & - & SUM & 100.00 & 24.22 & 23.28 & 22.34 & 24.68 & 23.72 & 22.76 \\
\hline
\end{tabular}


Table 3.5. Composition of Glasses Tested for LAW AP-101 (per PNNL-13354 [8]).

\begin{tabular}{|c|c|c|c|c|c|c|c|c|c|c|c|c|}
\hline $\begin{array}{l}\text { Glass } \\
\text { Oxides }\end{array}$ & $\begin{array}{c}\text { LAW } \\
\text { AP-101 } \\
\text { pretreated }\end{array}$ & $\begin{array}{c}\text { Glass } \\
\text { Former } \\
\text { Additives } \\
\text { for } \\
\text { LAWA130 }\end{array}$ & $\begin{array}{c}\text { LAWA130 } \\
\text { (target) }\end{array}$ & $\begin{array}{c}\text { LAW } \\
\text { AP-101 } \\
\text { pretreated }\end{array}$ & $\begin{array}{c}\text { Glass } \\
\text { Former } \\
\text { Additives } \\
\text { for } \\
\text { LAWA134 }\end{array}$ & $\begin{array}{c}\text { LAWA134 } \\
\text { (target) }\end{array}$ & $\begin{array}{c}\text { LAW } \\
\text { AP-101 } \\
\text { pretreated }\end{array}$ & $\begin{array}{c}\text { Glass } \\
\text { Former } \\
\text { Additives } \\
\text { for } \\
\text { LAWA135 }\end{array}$ & $\begin{array}{c}\text { LAWA135 } \\
\text { (target) }\end{array}$ & $\begin{array}{c}\text { LAW } \\
\text { AP-101 } \\
\text { pretreated }\end{array}$ & $\begin{array}{c}\text { Glass } \\
\text { Former } \\
\text { Additives } \\
\text { for } \\
\text { LAWA136 }\end{array}$ & $\begin{array}{l}\text { LAWA136 } \\
\text { (target) }\end{array}$ \\
\hline Loading & $24.68 \%$ & - & $24.68 \%$ & $23.72 \%$ & - & $23.72 \%$ & $22.76 \%$ & - & $22.76 \%$ & $22.76 \%$ & - & $22.76 \%$ \\
\hline $\mathrm{Al}_{2} \mathrm{O}_{3}$ & 1.35 & 6.22 & 6.04 & 1.30 & 5.71 & 5.65 & 1.25 & 5.71 & 5.66 & 1.25 & 5.71 & 5.66 \\
\hline $\mathrm{B}_{2} \mathrm{O}_{3}$ & 0.01 & 11.90 & 8.97 & 0.01 & 13.07 & 9.97 & 0.01 & 13.07 & 10.10 & 0.01 & 13.07 & 10.10 \\
\hline $\mathrm{CaO}$ & - & 2.77 & 2.08 & - & 2.65 & 2.02 & - & 2.65 & 2.05 & - & 3.94 & 3.05 \\
\hline $\mathrm{Cr}_{2} \mathrm{O}_{3}$ & 0.02 & - & 0.02 & 0.02 & - & 0.02 & 0.02 & - & 0.02 & 0.02 & - & 0.02 \\
\hline $\mathrm{Fe}_{2} \mathrm{O}_{3}$ & - & 3.81 & 2.87 & - & 7.38 & 5.63 & - & 7.38 & 5.70 & - & 7.38 & 5.70 \\
\hline $\mathrm{K}_{2} \mathrm{O}$ & 3.88 & - & 3.88 & 3.73 & - & 3.73 & 3.58 & - & 3.58 & 3.58 & - & 3.58 \\
\hline $\mathrm{MgO}$ & - & 1.57 & 1.18 & - & 1.97 & 1.50 & - & 1.97 & 1.52 & - & 1.97 & 1.52 \\
\hline $\mathrm{Na}_{2} \mathrm{O}$ & 18.46 & - & 18.46 & 17.74 & - & 17.74 & 17.03 & - & 17.03 & 17.03 & - & 17.03 \\
\hline $\mathrm{SiO}_{2}$ & 0.03 & 61.29 & 46.20 & 0.03 & 58.67 & 44.78 & 0.03 & 58.67 & 45.34 & 0.03 & 57.37 & 44.34 \\
\hline $\mathrm{TiO}_{2}$ & - & 2.77 & 2.09 & - & 2.66 & 2.03 & - & 2.66 & 2.05 & - & 2.66 & 2.05 \\
\hline $\mathrm{ZnO}$ & - & 5.51 & 4.15 & - & 3.93 & 3.00 & - & 3.93 & 3.04 & - & 3.93 & 3.04 \\
\hline $\mathrm{ZrO}_{2}$ & - & 4.16 & 3.13 & - & 3.98 & 3.04 & - & 3.98 & 3.08 & - & 3.98 & 3.08 \\
\hline $\mathrm{Cl}$ & 0.20 & - & 0.20 & 0.20 & - & 0.20 & 0.19 & - & 0.19 & 0.19 & - & 0.19 \\
\hline $\mathrm{F}$ & 0.30 & - & 0.30 & 0.29 & - & 0.29 & 0.28 & - & 0.28 & 0.28 & - & 0.28 \\
\hline $\mathrm{P}_{2} \mathrm{O}_{5}$ & 0.08 & - & 0.08 & 0.08 & - & 0.08 & 0.07 & - & 0.07 & 0.07 & - & 0.07 \\
\hline $\mathrm{SO}_{3}$ & 0.35 & - & 0.35 & 0.33 & - & 0.33 & 0.32 & - & 0.32 & 0.32 & - & 0.32 \\
\hline $\mathrm{Re}_{2} \mathrm{O}_{7}$ & - & - & 0.10 & - & - & 0.10 & - & - & 0.10 & - & - & 0.10 \\
\hline SUM & 24.68 & 100.00 & 100.10 & 23.72 & 100.00 & 100.10 & 22.76 & 100.00 & 100.10 & 22.76 & 100.00 & 100.10 \\
\hline
\end{tabular}


Table 3.6. Compositions of LAW AW-101 Waste (Per TFCOUP Rev. 2) and the Glasses Tested.

\begin{tabular}{|c|c|c|c|c|c|c|c|c|c|c|c|c|}
\hline $\begin{array}{l}\text { Glass } \\
\text { Oxides }\end{array}$ & $\begin{array}{c}\text { LAW } \\
\text { AW-101 } \\
\text { Batch } 14\end{array}$ & $\begin{array}{c}\text { LAW } \\
\text { AW-101 } \\
\text { Batch } 15\end{array}$ & $\begin{array}{c}\text { LAW } \\
\text { AW-101 } \\
\text { Batch } 14 \\
\text { in Glass }\end{array}$ & $\begin{array}{c}\text { LAW } \\
\text { AW-101 } \\
\text { Batch } 15 \\
\text { in Glass }\end{array}$ & $\begin{array}{c}\text { LAW } \\
\text { AW-101 } \\
\text { Batch } 14 \\
\text { Pretreated } \\
\text { in Glass }\end{array}$ & $\begin{array}{c}\text { LAW } \\
\text { AW-101 } \\
\text { Batch } 15 \\
\text { Pretreated } \\
\text { In Glass }\end{array}$ & $\begin{array}{c}\text { Glass } \\
\text { Former } \\
\text { Additives } \\
\text { for } \\
\text { LAWA88 }\end{array}$ & $\begin{array}{c}\text { Glass } \\
\text { Former } \\
\text { Additives } \\
\text { for } \\
\text { LAWA112 }\end{array}$ & $\begin{array}{c}\text { Additives } \\
\text { for } \\
\text { LAWA112 } \\
\text { Batch } 14\end{array}$ & $\begin{array}{c}\text { LAWA112 } \\
\text { Batch } 14 \\
\text { (target) }\end{array}$ & $\begin{array}{c}\text { Additives } \\
\text { for } \\
\text { LAWA112 } \\
\text { Batch } 15\end{array}$ & $\begin{array}{c}\text { LAWA112 } \\
\text { Batch } 15 \\
\text { (target) }\end{array}$ \\
\hline Loading & - & - & $25.29 \%$ & $25.71 \%$ & $25.79 \%$ & $26.21 \%$ & - & - & $74.21 \%$ & $25.79 \%$ & $73.79 \%$ & $26.21 \%$ \\
\hline $\mathrm{Al}_{2} \mathrm{O}_{3}$ & 12.36 & 12.47 & 3.13 & 3.21 & 3.13 & 3.21 & 4.25 & 4.00 & 2.97 & 6.10 & 2.95 & 6.16 \\
\hline $\mathrm{B}_{2} \mathrm{O}_{3}$ & 0.00 & 0.00 & 0.00 & 0.00 & 0.00 & 0.00 & 13.15 & 13.30 & 9.87 & 9.87 & 9.81 & 9.81 \\
\hline $\mathrm{CaO}$ & 0.03 & 0.02 & 0.01 & 0.01 & 0.01 & 0.01 & 2.70 & 10.30 & 7.64 & 7.65 & 7.60 & 7.61 \\
\hline $\mathrm{Cr}_{2} \mathrm{O}_{3}$ & 0.07 & 0.05 & 0.02 & 0.01 & 0.02 & 0.01 & - & - & - & 0.02 & - & 0.01 \\
\hline $\mathrm{Fe}_{2} \mathrm{O}_{3}$ & 0.01 & 0.01 & 0.00 & 0.00 & 0.00 & 0.00 & 7.50 & 0.00 & 0.00 & 0.00 & 0.00 & 0.00 \\
\hline $\mathrm{K}_{2} \mathrm{O}$ & 7.48 & 9.38 & 1.89 & 2.41 & 1.89 & 2.41 & - & - & - & 1.89 & - & 2.41 \\
\hline $\mathrm{Li}_{2} \mathrm{O}$ & 0.00 & 0.00 & 0.00 & 0.00 & 0.00 & 0.00 & - & - & - & 0.00 & - & 0.00 \\
\hline $\mathrm{MgO}$ & 0.00 & 0.00 & 0.00 & 0.00 & 0.00 & 0.00 & 2.00 & 2.00 & 1.48 & 1.48 & 1.48 & 1.48 \\
\hline $\mathrm{Na}_{2} \mathrm{O}$ & 77.09 & 75.85 & 19.50 & 19.50 & 20.00 & 20.00 & - & - & - & 20.00 & - & 20.00 \\
\hline $\mathrm{SiO}_{2}$ & 0.00 & 0.00 & 0.00 & 0.00 & 0.00 & 0.00 & 59.65 & 59.65 & 44.26 & 44.26 & 44.02 & 44.02 \\
\hline $\mathrm{TiO}_{2}$ & 0.00 & 0.00 & 0.00 & 0.00 & 0.00 & 0.00 & 2.70 & 2.70 & 2.00 & 2.00 & 1.99 & 1.99 \\
\hline $\mathrm{ZnO}$ & 0.00 & 0.00 & 0.00 & 0.00 & 0.00 & 0.00 & 4.00 & 4.00 & 2.97 & 2.97 & 2.95 & 2.95 \\
\hline $\mathrm{ZrO}_{2}$ & 0.00 & 0.00 & 0.00 & 0.00 & 0.00 & 0.00 & 4.05 & 4.05 & 3.01 & 3.01 & 2.99 & 2.99 \\
\hline $\mathrm{Cl}$ & 1.49 & 1.21 & 0.38 & 0.31 & 0.38 & 0.31 & - & - & - & 0.38 & - & 0.31 \\
\hline $\mathrm{F}$ & 0.38 & 0.37 & 0.10 & 0.09 & 0.10 & 0.09 & - & - & - & 0.10 & - & 0.09 \\
\hline $\mathrm{P}_{2} \mathrm{O}_{5}$ & 0.39 & 0.20 & 0.10 & 0.05 & 0.10 & 0.05 & - & - & 0.00 & 0.10 & 0.00 & 0.05 \\
\hline $\mathrm{SO}_{3}$ & 0.68 & 0.42 & 0.17 & 0.11 & 0.17 & 0.11 & - & - & - & 0.17 & - & 0.11 \\
\hline $\mathrm{Re}_{2} \mathrm{O}_{7}$ & - & - & - & - & - & - & - & - & - & 0.10 & - & 0.10 \\
\hline SUM & 100.00 & 100.00 & 25.29 & 25.71 & 25.79 & 26.21 & 100.00 & 100.00 & 74.21 & 100.10 & 73.79 & 100.10 \\
\hline
\end{tabular}


Table 3.7. Composition of LAW AN-104 (Batch 5) Per TFCOUP Rev. 2 and Composition of LAWA102 Glass.

\begin{tabular}{|c|c|c|c|c|c|c|c|c|}
\hline $\begin{array}{l}\text { Glass } \\
\text { Oxides }\end{array}$ & $\begin{array}{c}\text { LAW } \\
\text { AN-104 } \\
\text { Batch } 5\end{array}$ & $\begin{array}{c}\text { LAW } \\
\text { AN-104 } \\
\text { in Glass } \\
\text { wt\% }\end{array}$ & $\begin{array}{c}\text { LAW } \\
\text { AN-104 } \\
\text { Pretreated } \\
\text { wt\% }\end{array}$ & $\begin{array}{c}\text { Recycled } \\
\text { off gas } \\
\mathrm{SO}_{3}\end{array}$ & $\begin{array}{c}\text { Pretreated } \\
\text { LAW AN- } \\
104 \\
\text { + recycled } \\
\mathrm{SO}_{3}\end{array}$ & $\begin{array}{c}\text { Glass } \\
\text { Former } \\
\text { Additives } \\
\text { for } \\
\text { LAWA102 }\end{array}$ & $\begin{array}{c}\text { Glass } \\
\text { Former } \\
\text { Additives } \\
\text { for } \\
\text { LAWA102 }\end{array}$ & $\begin{array}{c}\text { LAWA102 } \\
\text { Batch } 5 \\
\text { (target) }\end{array}$ \\
\hline Loading & - & $17.80 \%$ & $18.16 \%$ & - & $18.32 \%$ & $100.00 \%$ & $81.68 \%$ & $18.32 \%$ \\
\hline $\mathrm{Al}_{2} \mathrm{O}_{3}$ & 14.75 & 2.63 & 2.63 & - & 2.63 & 4.19 & 3.42 & 6.05 \\
\hline $\mathrm{B}_{2} \mathrm{O}_{3}$ & 0.00 & 0.00 & 0.00 & - & 0.00 & 12.25 & 10.01 & 10.01 \\
\hline $\mathrm{CaO}$ & 0.01 & 0.00 & 0.00 & - & 0.00 & 6.20 & 5.06 & 5.07 \\
\hline $\mathrm{Cr}_{2} \mathrm{O}_{3}$ & 0.10 & 0.02 & 0.02 & - & 0.02 & - & - & 0.02 \\
\hline $\mathrm{Fe}_{2} \mathrm{O}_{3}$ & 0.01 & 0.00 & 0.00 & - & 0.00 & 6.62 & 5.41 & 5.41 \\
\hline $\mathrm{K}_{2} \mathrm{O}$ & 1.46 & 0.26 & 0.26 & - & 0.26 & - & - & 0.26 \\
\hline $\mathrm{Li}_{2} \mathrm{O}$ & 0.00 & 0.00 & 0.00 & - & 0.00 & 3.06 & 2.50 & 2.50 \\
\hline $\mathrm{MgO}$ & 0.00 & 0.00 & 0.00 & - & 0.00 & 1.83 & 1.49 & 1.49 \\
\hline $\mathrm{Na}_{2} \mathrm{O}$ & 78.92 & 14.05 & 14.41 & 0.07 & 14.48 & - & - & 14.48 \\
\hline $\mathrm{SiO}_{2}$ & 0.00 & 0.00 & 0.00 & - & 0.00 & 57.00 & 46.56 & 46.56 \\
\hline $\mathrm{TiO}_{2}$ & 0.00 & 0.00 & 0.00 & - & 0.00 & 1.40 & 1.14 & 1.14 \\
\hline $\mathrm{ZnO}$ & 0.00 & 0.00 & 0.00 & - & 0.00 & 3.75 & 3.06 & 3.06 \\
\hline $\mathrm{ZrO}_{2}$ & 0.00 & 0.00 & 0.00 & - & 0.00 & 3.70 & 3.02 & 3.02 \\
\hline $\mathrm{Cl}$ & 1.87 & 0.33 & 0.33 & - & 0.33 & - & - & 0.33 \\
\hline $\mathrm{F}$ & 0.14 & 0.03 & 0.03 & - & 0.03 & - & - & 0.03 \\
\hline $\mathrm{P}_{2} \mathrm{O}_{5}$ & 0.75 & 0.13 & 0.13 & - & 0.13 & - & - & 0.13 \\
\hline $\mathrm{SO}_{3}$ & 1.97 & 0.35 & 0.35 & 0.09 & 0.44 & - & - & 0.44 \\
\hline SUM & 100.00 & 17.80 & 18.16 & 0.16 & 18.32 & 100.00 & 81.68 & 100.00 \\
\hline
\end{tabular}


Table 3.7 (continued). Composition of Glasses Tested for LAW AN-104 (Batch 5) Per TFCOUP Rev. 2 (cont'd).

\begin{tabular}{|c|c|c|c|c|c|c|c|c|c|c|c|}
\hline \multirow{2}{*}{$\begin{array}{c}\text { Glass } \\
\text { Oxides } \\
\text { Loading } \\
\end{array}$} & \multirow{2}{*}{$\begin{array}{c}\text { Pretreated } \\
\text { AN-104 } \\
+\begin{array}{c}\text { recycled } \\
\text { SO3 }\end{array} \\
18.32 \% \\
\end{array}$} & \multirow{2}{*}{$\begin{array}{l}\text { Delta } \\
\text { To } \\
\text { LAW } \\
\text { A102 } \\
- \\
\end{array}$} & \multicolumn{2}{|c|}{$\begin{array}{l}\text { Glass Former } \\
\text { Additives for } \\
\text { LAWA118 }\end{array}$} & \multirow{2}{*}{$\begin{array}{c}\begin{array}{c}\text { LAWA118S1 } \\
\text { (target) }\end{array} \\
18.32 \%\end{array}$} & \multirow{2}{*}{$\begin{array}{c}\begin{array}{c}\text { LAWA118S2 } \\
\text { (target) }\end{array} \\
18.32 \% \\
\end{array}$} & \multirow{2}{*}{$\begin{array}{c}\text { Delta } \\
\text { To } \\
\text { LAW } \\
\text { A102 } \\
-\end{array}$} & \multicolumn{2}{|c|}{$\begin{array}{l}\text { Glass Former Additives } \\
\text { for LAWA119 }\end{array}$} & \multirow{2}{*}{$\begin{array}{c}\begin{array}{c}\text { LAWA119S1 } \\
\text { (target) }\end{array} \\
18.32 \%\end{array}$} & \multirow{2}{*}{$\begin{array}{c}\begin{array}{c}\text { LAWA119S2 } \\
\text { (target) }\end{array} \\
18.32 \% \\
\end{array}$} \\
\hline & & & $100 \%$ & $81.68 \%$ & & & & $100 \%$ & $81.68 \%$ & & \\
\hline $\mathrm{Al}_{2} \mathrm{O}_{3}$ & 2.63 & - & 4.19 & 3.42 & 6.05 & 6.05 & - & 4.19 & 3.42 & 6.05 & 6.05 \\
\hline $\mathrm{B}_{2} \mathrm{O}_{3}$ & 0.00 & - & 12.25 & 10.01 & 10.01 & 10.01 & - & 12.25 & 10.01 & 10.01 & 10.01 \\
\hline $\mathrm{CaO}$ & 0.00 & - & 6.20 & 5.06 & 5.07 & 5.07 & 5.41 & 12.82 & 10.47 & 10.48 & 10.48 \\
\hline $\mathrm{Cr}_{2} \mathrm{O}_{3}$ & 0.02 & - & - & 0.00 & 0.02 & 0.02 & - & - & 0.00 & 0.02 & 0.02 \\
\hline $\mathrm{Fe}_{2} \mathrm{O}_{3}$ & 0.00 & - & 6.62 & 5.41 & 5.41 & 5.41 & -5.41 & 0.00 & 0.00 & 0.00 & 0.00 \\
\hline $\mathrm{K}_{2} \mathrm{O}$ & 0.26 & 2.50 & 3.06 & 2.50 & 2.76 & 2.76 & 2.50 & 3.06 & 2.50 & 2.76 & 2.76 \\
\hline $\mathrm{Li}_{2} \mathrm{O}$ & 0.00 & -2.50 & - & 0.00 & 0.00 & 0.00 & -2.50 & - & 0.00 & 0.00 & 0.00 \\
\hline $\mathrm{MgO}$ & 0.00 & - & 1.83 & 1.49 & 1.49 & 1.49 & - & 1.83 & 1.49 & 1.49 & 1.49 \\
\hline $\mathrm{Na}_{2} \mathrm{O}$ & 14.48 & - & - & - & 14.48 & 14.48 & - & & & 14.48 & 14.48 \\
\hline $\mathrm{SiO}_{2}$ & 0.00 & - & 57.00 & 46.56 & 46.56 & 46.56 & - & 57.00 & 46.56 & 46.56 & 46.56 \\
\hline $\mathrm{TiO}_{2}$ & 0.00 & - & 1.40 & 1.14 & 1.14 & 1.14 & - & 1.40 & 1.14 & 1.14 & 1.14 \\
\hline $\mathrm{ZnO}$ & 0.00 & - & 3.75 & 3.06 & 3.06 & 3.06 & - & 3.75 & 3.06 & 3.06 & 3.06 \\
\hline $\mathrm{ZrO}_{2}$ & 0.00 & - & 3.70 & 3.02 & 3.02 & 3.02 & - & 3.70 & 3.02 & 3.02 & 3.02 \\
\hline $\mathrm{Cl}$ & 0.33 & - & - & - & 0.33 & 0.33 & - & - & - & 0.33 & 0.33 \\
\hline $\mathrm{F}$ & 0.03 & - & - & - & 0.03 & 0.03 & - & - & - & 0.03 & 0.03 \\
\hline $\mathrm{P}_{2} \mathrm{O}_{5}$ & 0.13 & - & - & - & 0.13 & 0.13 & - & - & - & 0.13 & 0.13 \\
\hline $\mathrm{SO}_{3}$ & 0.44 & - & - & - & 1.48 & 2.50 & - & - & - & 1.48 & 2.50 \\
\hline $\mathrm{ReO}_{2}$ & - & - & - & - & 0.10 & 0.10 & - & - & - & 0.10 & 0.10 \\
\hline SUM & 18.32 & 0.00 & 100.00 & 81.68 & 101.14 & 102.16 & 0.00 & 100.00 & 81.68 & 101.14 & 102.16 \\
\hline
\end{tabular}


Table 3.7 Composition of Glasses Tested for LAW AN-104 (Batch 5) Per TFCOUP Rev. 2 (cont'd).

\begin{tabular}{|c|c|c|c|c|c|c|c|c|c|c|c|}
\hline \multirow{2}{*}{$\begin{array}{c}\text { Glass } \\
\text { Oxides } \\
\text { Loading }\end{array}$} & \multirow{2}{*}{$\begin{array}{c}\text { Pretreated } \\
\text { LAW } \\
\text { AN-104 } \\
+ \text { recycled } \\
\mathrm{SO}_{3} \\
18.32 \% \\
\end{array}$} & \multirow{2}{*}{$\begin{array}{l}\text { Delta } \\
\text { To } \\
\text { LAW } \\
\text { A102 } \\
- \\
\end{array}$} & \multicolumn{2}{|c|}{$\begin{array}{l}\text { Glass Former } \\
\text { Additives for } \\
\text { LAWA120 }\end{array}$} & \multirow{2}{*}{$\begin{array}{c}\begin{array}{c}\text { LAWA120S1 } \\
\text { (target) }\end{array} \\
18.32 \%\end{array}$} & \multirow{2}{*}{$\begin{array}{c}\text { LAWA120S2 } \\
\text { (target) } \\
18.32 \%\end{array}$} & \multirow{2}{*}{$\begin{array}{l}\text { Delta } \\
\text { To } \\
\text { LAW } \\
\text { A102 } \\
-\end{array}$} & \multicolumn{2}{|c|}{$\begin{array}{l}\text { Glass Former } \\
\text { Additives for } \\
\text { LAWA121 }\end{array}$} & \multirow{2}{*}{$\begin{array}{l}\begin{array}{c}\text { LAWA121S1 } \\
\text { (target) }\end{array} \\
18.32 \%\end{array}$} & \multirow{2}{*}{$\begin{array}{c}\begin{array}{c}\text { LAWA121S2 } \\
\text { (target) }\end{array} \\
18.32 \%\end{array}$} \\
\hline & & & $100 \%$ & $81.68 \%$ & & & & $100 \%$ & $81.68 \%$ & & \\
\hline $\mathrm{Al}_{2} \mathrm{O}_{3}$ & 2.63 & -0.23 & 3.91 & 3.19 & 5.82 & 5.82 & -0.23 & 3.91 & 3.19 & 5.82 & 5.82 \\
\hline $\mathrm{B}_{2} \mathrm{O}_{3}$ & 0.00 & -0.70 & 11.38 & 9.30 & 9.30 & 9.30 & -0.70 & 11.38 & 9.30 & 9.30 & 9.30 \\
\hline $\mathrm{CaO}$ & 0.00 & -0.36 & 5.77 & 4.71 & 4.71 & 4.71 & 4.68 & 11.92 & 9.74 & 9.74 & 9.74 \\
\hline $\mathrm{Cr}_{2} \mathrm{O}_{3}$ & 0.02 & - & - & 0.00 & 0.02 & 0.02 & - & - & 0.00 & 0.02 & 0.02 \\
\hline $\mathrm{Fe}_{2} \mathrm{O}_{3}$ & 0.00 & -0.38 & 6.16 & 5.03 & 5.03 & 5.03 & -5.41 & 0.00 & 0.00 & 0.00 & 0.00 \\
\hline $\mathrm{K}_{2} \mathrm{O}$ & 0.26 & 8.05 & 9.86 & 8.05 & 8.31 & 8.31 & 8.05 & 9.86 & 8.05 & 8.31 & 8.31 \\
\hline $\mathrm{Li}_{2} \mathrm{O}$ & 0.00 & -2.50 & - & 0.00 & 0.00 & 0.00 & -2.50 & & 0.00 & 0.00 & 0.00 \\
\hline $\mathrm{MgO}$ & 0.00 & -0.10 & 1.70 & 1.39 & 1.39 & 1.39 & -0.10 & 1.70 & 1.39 & 1.39 & 1.39 \\
\hline $\mathrm{Na}_{2} \mathrm{O}$ & 14.48 & - & - & 0.00 & 14.48 & 14.48 & - & - & - & 14.48 & 14.48 \\
\hline $\mathrm{SiO}_{2}$ & 0.00 & -3.27 & 53.00 & 43.29 & 43.29 & 43.29 & -3.27 & 53.00 & 43.29 & 43.29 & 43.29 \\
\hline $\mathrm{TiO}_{2}$ & 0.00 & -0.08 & 1.30 & 1.06 & 1.06 & 1.06 & -0.08 & 1.30 & 1.06 & 1.06 & 1.06 \\
\hline $\mathrm{ZnO}$ & 0.00 & -0.21 & 3.49 & 2.85 & 2.85 & 2.85 & -0.21 & 3.49 & 2.85 & 2.85 & 2.85 \\
\hline $\mathrm{ZrO}_{2}$ & 0.00 & -0.21 & 3.44 & 2.81 & 2.81 & 2.81 & -0.21 & 3.44 & 2.81 & 2.81 & 2.81 \\
\hline $\mathrm{Cl}$ & 0.33 & - & - & - & 0.33 & 0.33 & - & - & - & 0.33 & 0.33 \\
\hline $\mathrm{F}$ & 0.03 & - & - & - & 0.03 & 0.03 & - & - & - & 0.03 & 0.03 \\
\hline $\mathrm{P}_{2} \mathrm{O}_{5}$ & 0.13 & - & - & - & 0.13 & 0.13 & - & - & - & 0.13 & 0.13 \\
\hline $\mathrm{SO}_{3}$ & 0.44 & - & - & - & 1.48 & 2.50 & - & - & - & 1.48 & 2.50 \\
\hline $\mathrm{ReO}_{2}$ & - & - & - & - & 0.10 & 0.10 & - & - & - & 0.10 & 0.10 \\
\hline SUM & 18.32 & - & 100.00 & 81.68 & 101.15 & 102.17 & - & 100.00 & 81.68 & 101.15 & 102.17 \\
\hline
\end{tabular}


Table 3.7 Composition of Glasses Tested for LAW AN-104 (Batch 5) Per TFCOUP Rev. 2 (cont'd).

\begin{tabular}{|c|c|c|c|c|c|c|c|c|c|c|c|}
\hline \multirow{2}{*}{$\begin{array}{c}\text { Glass } \\
\text { Oxides } \\
\text { Loading }\end{array}$} & \multirow{2}{*}{$\begin{array}{c}\text { Pretreated } \\
\text { LAW } \\
\text { AN-104 } \\
+ \text { recycled } \\
\text { SO3 } \\
18.32 \% \\
\end{array}$} & \multirow{2}{*}{$\begin{array}{l}\text { Delta } \\
\text { To } \\
\text { LAW } \\
\text { A102 } \\
-\end{array}$} & \multicolumn{2}{|c|}{$\begin{array}{l}\text { Glass Former Additives } \\
\text { for LAWA122 }\end{array}$} & \multirow{2}{*}{$\begin{array}{l}\begin{array}{c}\text { LAWA122S1 } \\
\text { (target) }\end{array} \\
18.32 \%\end{array}$} & \multirow{2}{*}{$\begin{array}{c}\begin{array}{c}\text { LAWA122S2 } \\
\text { (target) }\end{array} \\
18.32 \%\end{array}$} & \multirow[t]{2}{*}{$\begin{array}{l}\text { Delta } \\
\text { To } \\
\text { LAW } \\
\text { A102 }\end{array}$} & \multicolumn{2}{|c|}{$\begin{array}{c}\text { Glass Former } \\
\text { Additives for } \\
\text { LAWA123 }\end{array}$} & \multirow{2}{*}{$\begin{array}{c}\text { LAWA123S1 } \\
\text { (target) } \\
18.32 \%\end{array}$} & \multirow{2}{*}{$\begin{array}{c}\text { LAWA123S2 } \\
\text { (target) } \\
\\
18.32 \%\end{array}$} \\
\hline & & & $100 \%$ & $81.68 \%$ & & & & $100 \%$ & $81.68 \%$ & & \\
\hline $\mathrm{Al}_{2} \mathrm{O}_{3}$ & 2.63 & -0.12 & 4.05 & 3.31 & 5.93 & 5.93 & -0.12 & 4.05 & 3.31 & 5.93 & 5.93 \\
\hline $\mathrm{B}_{2} \mathrm{O}_{3}$ & 0.00 & -0.36 & 11.80 & 9.64 & 9.64 & 9.64 & -0.36 & 11.80 & 9.64 & 9.64 & 9.64 \\
\hline $\mathrm{CaO}$ & 0.00 & -0.18 & 5.97 & 4.88 & 4.88 & 4.88 & 5.03 & 12.35 & 10.09 & 10.10 & 10.10 \\
\hline $\mathrm{Cr}_{2} \mathrm{O}_{3}$ & 0.02 & - & - & 0.00 & 0.02 & 0.02 & - & - & 0.00 & 0.02 & 0.02 \\
\hline $\mathrm{Fe}_{2} \mathrm{O}_{3}$ & 0.00 & -0.20 & 6.38 & 5.21 & 5.21 & 5.21 & -5.41 & 0.00 & 0.00 & 0.00 & 0.00 \\
\hline $\mathrm{K}_{2} \mathrm{O}$ & 0.26 & 5.38 & 6.58 & 5.38 & 5.64 & 5.64 & 5.38 & 6.58 & 5.38 & 5.64 & 5.64 \\
\hline $\mathrm{Li}_{2} \mathrm{O}$ & 0.00 & -2.50 & - & 0.00 & 0.00 & 0.00 & -2.50 & - & 0.00 & 0.00 & 0.00 \\
\hline $\mathrm{MgO}$ & 0.00 & -0.05 & 1.76 & 1.44 & 1.44 & 1.44 & -0.05 & 1.76 & 1.44 & 1.44 & 1.44 \\
\hline $\mathrm{Na}_{2} \mathrm{O}$ & 14.48 & - & - & 0.00 & 14.48 & 14.48 & - & - & 0.00 & 14.48 & 14.48 \\
\hline $\mathrm{SiO}_{2}$ & 0.00 & -1.69 & 54.93 & 44.87 & 44.87 & 44.87 & -1.69 & 54.93 & 44.87 & 44.87 & 44.87 \\
\hline $\mathrm{TiO}_{2}$ & 0.00 & -0.04 & 1.35 & 1.10 & 1.10 & 1.10 & -0.04 & 1.35 & 1.10 & 1.10 & 1.10 \\
\hline $\mathrm{ZnO}$ & 0.00 & -0.11 & 3.61 & 2.95 & 2.95 & 2.95 & -0.11 & 3.61 & 2.95 & 2.95 & 2.95 \\
\hline $\mathrm{ZrO}_{2}$ & 0.00 & -0.11 & 3.57 & 2.91 & 2.91 & 2.91 & -0.11 & 3.57 & 2.91 & 2.91 & 2.91 \\
\hline $\mathrm{Cl}$ & 0.33 & - & - & - & 0.33 & 0.33 & - & - & - & 0.33 & 0.33 \\
\hline $\mathrm{F}$ & 0.03 & - & - & - & 0.03 & 0.03 & - & - & - & 0.03 & 0.03 \\
\hline $\mathrm{P}_{2} \mathrm{O}_{5}$ & 0.13 & - & - & - & 0.13 & 0.13 & - & - & - & 0.13 & 0.13 \\
\hline $\mathrm{SO}_{3}$ & 0.44 & - & - & - & 1.48 & 2.50 & - & - & - & 1.48 & 2.50 \\
\hline $\mathrm{ReO}_{2}$ & - & - & - & - & 0.10 & 0.10 & - & - & - & 0.10 & 0.10 \\
\hline SUM & 18.32 & - & 100.00 & 81.68 & 101.15 & 102.17 & - & 100.00 & 81.68 & 101.15 & 102.17 \\
\hline
\end{tabular}


Table 3.8. Composition of LAW AZ-101 (Batch 2a) Per TFCOUP Rev. 2 and Waste Contribution to Glass.

\begin{tabular}{|c|c|c|c|c|c|c|c|c|c|}
\hline \multirow[b]{2}{*}{$\begin{array}{l}\text { Glass } \\
\text { Oxides }\end{array}$} & \multirow[b]{2}{*}{$\begin{array}{c}\text { LAW } \\
\text { AZ-101 } \\
\text { Batch 2a }\end{array}$} & \multicolumn{4}{|c|}{$\begin{array}{l}\text { LAW AZ-101 at a waste loading of } 6.17 \text { wt\% } \\
\text { Na2O }\end{array}$} & \multicolumn{4}{|c|}{$\begin{array}{l}\text { LAW AZ-101 at a waste loading of } 5.1 \mathrm{wt} \% \\
\text { Na2O }\end{array}$} \\
\hline & & $\begin{array}{c}\text { Waste } \\
\text { Contribution } \\
\text { to Glass } \\
\text { wt } \%\end{array}$ & $\begin{array}{l}\text { Including } \\
\text { Pre- } \\
\text { treatment }\end{array}$ & $\begin{array}{c}\text { Recycled } \\
\text { Off-gas } \\
\mathrm{SO}_{3}\end{array}$ & $\begin{array}{c}\text { Pre- } \\
\text { treatment } \\
+ \\
\text { recycled } \\
\mathrm{SO}_{3} \\
\end{array}$ & $\begin{array}{c}\text { Waste } \\
\text { Contribution } \\
\text { to Glass } \\
\text { wt\% }\end{array}$ & $\begin{array}{c}\text { Including } \\
\text { Pre- } \\
\text { treatment }\end{array}$ & $\begin{array}{c}\text { Recycled } \\
\text { Off-gas } \\
\mathrm{SO}_{3}\end{array}$ & $\begin{array}{c}\text { Pre- } \\
\text { treatment } \\
+ \\
\text { recycled } \\
\mathrm{SO}_{3} \\
\end{array}$ \\
\hline Loading & $100 \%$ & $8.06 \%$ & $8.39 \%$ & - & $8.67 \%$ & $6.66 \%$ & $6.94 \%$ & - & $7.17 \%$ \\
\hline $\mathrm{Al}_{2} \mathrm{O}_{3}$ & 10.59 & 0.85 & 0.85 & - & 0.85 & 0.71 & 0.71 & - & 0.71 \\
\hline $\mathrm{B}_{2} \mathrm{O}_{3}$ & 0.00 & 0.00 & 0.00 & - & 0.00 & 0.00 & 0.00 & - & 0.00 \\
\hline $\mathrm{CaO}$ & 0.00 & 0.00 & 0.00 & - & 0.00 & 0.00 & 0.00 & - & 0.00 \\
\hline $\mathrm{Cr}_{2} \mathrm{O}_{3}$ & 0.58 & 0.05 & 0.05 & - & 0.05 & 0.04 & 0.04 & - & 0.04 \\
\hline $\mathrm{Fe}_{2} \mathrm{O}_{3}$ & 0.01 & 0.00 & 0.00 & - & 0.00 & 0.00 & 0.00 & - & 0.00 \\
\hline $\mathrm{K}_{2} \mathrm{O}$ & 2.90 & 0.23 & 0.23 & - & 0.23 & 0.19 & 0.19 & - & 0.19 \\
\hline $\mathrm{La}_{2} \mathrm{O}_{3}$ & 0.00 & 0.00 & 0.00 & - & 0.00 & 0.00 & 0.00 & - & 0.00 \\
\hline $\mathrm{Na}_{2} \mathrm{O}$ & 76.52 & 6.17 & 6.50 & 0.12 & 6.62 & 5.10 & 5.37 & 0.10 & 5.47 \\
\hline $\mathrm{Cl}$ & 0.10 & 0.01 & 0.01 & - & 0.01 & 0.01 & 0.01 & - & 0.01 \\
\hline $\mathrm{F}$ & 0.96 & 0.08 & 0.08 & - & 0.08 & 0.06 & 0.06 & - & 0.06 \\
\hline $\mathrm{P}_{2} \mathrm{O}_{5}$ & 0.56 & 0.05 & 0.05 & - & 0.05 & 0.04 & 0.04 & - & 0.04 \\
\hline $\mathrm{SO}_{3}$ & 7.76 & 0.63 & 0.63 & 0.16 & 0.78 & 0.52 & 0.52 & 0.13 & 0.65 \\
\hline SUM & 100.00 & 8.06 & 8.39 & 0.28 & 8.67 & 6.66 & 6.94 & 0.23 & 7.17 \\
\hline
\end{tabular}


Table 3.9. Composition of Glasses LAWB45 and LAWB60 for LAW AZ-101 Per TFCOUP Rev. 2 High Waste Loading.

\begin{tabular}{|c|c|c|c|c|c|c|c|c|}
\hline $\begin{array}{l}\text { Glass } \\
\text { Oxides }\end{array}$ & $\begin{array}{c}\text { Pretreated } \\
\text { LAW AZ-101 } \\
\text { + recycled } \\
\text { SO3 }\end{array}$ & \multicolumn{2}{|c|}{$\begin{array}{l}\text { Glass Former } \\
\text { Additives for } \\
\text { LAWB45 }\end{array}$} & \multirow{2}{*}{$\begin{array}{l}\begin{array}{c}\text { LAWB45 } \\
\text { (target) }\end{array} \\
8.67 \%\end{array}$} & \multirow{2}{*}{\begin{tabular}{|c|}
$\begin{array}{c}\text { Delta } \\
\text { to } \\
\text { LAWB45 } \\
-\end{array}$ \\
\end{tabular}} & \multicolumn{2}{|c|}{$\begin{array}{l}\text { Glass Former Additives } \\
\text { for LAWB60 }\end{array}$} & \multirow{2}{*}{$\begin{array}{c}\begin{array}{c}\text { LAWB60S4 } \\
\text { (target) }\end{array} \\
8.67 \%\end{array}$} \\
\hline Loading & $8.67 \%$ & $100.00 \%$ & $91.33 \%$ & & & $100.00 \%$ & $91.33 \%$ & \\
\hline $\mathrm{Al}_{2} \mathrm{O}_{3}$ & 0.85 & 5.80 & 5.30 & 6.15 & - & 5.80 & 5.30 & 6.15 \\
\hline $\mathrm{B}_{2} \mathrm{O}_{3}$ & 0.00 & 13.50 & 12.33 & 12.33 & - & 13.50 & 12.33 & 12.33 \\
\hline $\mathrm{CaO}$ & 0.00 & 7.25 & 6.62 & 6.62 & 5.25 & 13.00 & 11.87 & 11.87 \\
\hline $\mathrm{Cr}_{2} \mathrm{O}_{3}$ & 0.05 & - & 0.00 & 0.05 & - & - & - & 0.05 \\
\hline $\mathrm{Fe}_{2} \mathrm{O}_{3}$ & 0.00 & 5.75 & 5.25 & 5.25 & -5.25 & 0.00 & 0.00 & 0.00 \\
\hline $\mathrm{K}_{2} \mathrm{O}$ & 0.23 & - & 0.00 & 0.23 & - & - & - & 0.23 \\
\hline $\mathrm{Li}_{2} \mathrm{O}$ & - & 5.05 & 4.61 & 4.61 & - & 5.05 & 4.61 & 4.61 \\
\hline $\mathrm{MgO}$ & - & 3.25 & 2.97 & 2.97 & - & 3.25 & 2.97 & 2.97 \\
\hline $\mathrm{Na}_{2} \mathrm{O}$ & 6.62 & - & 0.00 & 6.62 & - & & & 6.62 \\
\hline $\mathrm{SiO}_{2}$ & - & 52.50 & 47.95 & 47.95 & - & 52.50 & 47.95 & 47.95 \\
\hline $\mathrm{TiO}_{2}$ & - & 0.00 & 0.00 & 0.00 & - & 0.00 & 0.00 & 0.00 \\
\hline $\mathrm{ZnO}$ & - & 3.45 & 3.15 & 3.15 & - & 3.45 & 3.15 & 3.15 \\
\hline $\mathrm{ZrO}_{2}$ & - & 3.45 & 3.15 & 3.15 & - & 3.45 & 3.15 & 3.15 \\
\hline $\mathrm{Cl}$ & 0.01 & - & - & 0.01 & - & - & - & 0.01 \\
\hline $\mathrm{F}$ & 0.08 & - & - & 0.08 & - & - & - & 0.08 \\
\hline $\mathrm{P}_{2} \mathrm{O}_{5}$ & 0.05 & - & - & 0.05 & - & - & - & 0.05 \\
\hline $\mathrm{SO}_{3}$ & 0.78 & - & - & 0.78 & - & - & - & 4.50 \\
\hline $\mathrm{Re}_{2} \mathrm{O}_{7}$ & - & - & - & 0.10 & - & - & - & 0.10 \\
\hline SUM & 8.67 & 100.00 & 91.33 & 100.10 & 0.00 & 100.00 & 91.33 & 103.82 \\
\hline
\end{tabular}


Table 3. 10. Composition of Glasses Tested for LAW AZ-101 Per TFCOUP Rev. 2 - High Waste Loading •

\begin{tabular}{|c|c|c|c|c|c|c|c|c|c|c|c|c|c|}
\hline \multirow{2}{*}{\begin{tabular}{|l} 
Glass \\
Oxides \\
Loading \\
\end{tabular}} & \multirow{2}{*}{ 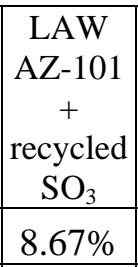 } & \multirow{2}{*}{\begin{tabular}{|c|} 
Delta to \\
LAW \\
B45 \\
- \\
\end{tabular}} & \multicolumn{2}{|c|}{$\begin{array}{c}\text { Glass Former } \\
\text { Additives for } \\
\text { LAWB69 }\end{array}$} & \multirow{2}{*}{$\begin{array}{c}\begin{array}{c}\text { LAWB69 } \\
\text { (target) }\end{array} \\
8.67 \% \\
\end{array}$} & \multirow{2}{*}{\begin{tabular}{|c|}
$\begin{array}{c}\text { LAWB69S4 } \\
\text { (target) }\end{array}$ \\
$8.67 \%$ \\
\end{tabular}} & \multirow{2}{*}{\begin{tabular}{|c|}
$\begin{array}{c}\text { LAWB69S0 } \\
\text { (target) }\end{array}$ \\
$8.67 \%$ \\
\end{tabular}} & \multirow{2}{*}{\begin{tabular}{|c} 
Delta \\
to \\
LAW \\
B45 \\
- \\
\end{tabular}} & \multicolumn{2}{|c|}{$\begin{array}{c}\text { Glass Former } \\
\text { Additives for } \\
\text { LAWB70 }\end{array}$} & \multirow{2}{*}{$\begin{array}{c}\begin{array}{c}\text { LAWB70 } \\
\text { (target) }\end{array} \\
8.67 \% \\
\end{array}$} & \multirow{2}{*}{\begin{tabular}{|c|}
$\begin{array}{c}\text { LAWB70S4 } \\
\text { (target) }\end{array}$ \\
$8.67 \%$ \\
\end{tabular}} & \multirow{2}{*}{\begin{tabular}{|c}
$\begin{array}{c}\text { LAWB70S0 } \\
\text { (target) }\end{array}$ \\
$8.67 \%$ \\
\end{tabular}} \\
\hline & & & $100 \%$ & $91.33 \%$ & & & & & $100 \%$ & $91.33 \%$ & & & \\
\hline $\mathrm{Al}_{2} \mathrm{O}_{3}$ & 0.85 & - & 5.80 & 5.30 & 6.15 & 6.15 & 6.15 & - & 5.80 & 5.30 & 6.15 & 6.15 & 6.15 \\
\hline $\mathrm{B}_{2} \mathrm{O}_{3}$ & 0.00 & - & 13.50 & 12.33 & 12.33 & 12.33 & 12.33 & - & 13.50 & 12.33 & 12.33 & 12.33 & 12.33 \\
\hline $\mathrm{CaO}$ & 0.00 & 3.84 & 11.45 & 10.46 & 10.46 & 10.46 & 10.46 & - & 7.25 & 6.62 & 6.62 & 6.62 & 6.62 \\
\hline $\mathrm{Cr}_{2} \mathrm{O}_{3}$ & 0.05 & - & - & - & 0.05 & 0.05 & 0.05 & - & - & - & 0.05 & 0.05 & 0.05 \\
\hline $\mathrm{Fe}_{2} \mathrm{O}_{3}$ & 0.00 & -5.25 & 0.00 & 0.00 & 0.00 & 0.00 & 0.00 & -2.00 & 3.56 & 3.25 & 3.25 & 3.25 & 3.25 \\
\hline $\mathrm{K}_{2} \mathrm{O}$ & 0.23 & - & - & - & 0.23 & 0.23 & 0.23 & - & - & - & 0.23 & 0.23 & 0.23 \\
\hline $\mathrm{Li}_{2} \mathrm{O}$ & - & - & 5.05 & 4.61 & 4.61 & 4.61 & 4.61 & - & 5.05 & 4.61 & 4.61 & 4.61 & 4.61 \\
\hline $\mathrm{MgO}$ & - & - & 3.25 & 2.97 & 2.97 & 2.97 & 2.97 & - & 3.25 & 2.97 & 2.97 & 2.97 & 2.97 \\
\hline $\mathrm{Na}_{2} \mathrm{O}$ & 6.62 & - & - & - & 6.62 & 6.62 & 6.62 & - & - & - & 6.62 & 6.62 & 6.62 \\
\hline $\mathrm{SiO}_{2}$ & - & - & 52.50 & 47.95 & 47.95 & 47.95 & 47.95 & - & 52.50 & 47.95 & 47.95 & 47.95 & 47.95 \\
\hline $\mathrm{TiO}_{2}$ & - & - & 0.00 & 0.00 & 0.00 & 0.00 & 0.00 & - & 0.00 & 0.00 & 0.00 & 0.00 & 0.00 \\
\hline $\mathrm{ZnO}$ & - & 1.42 & 5.00 & 4.57 & 4.57 & 4.57 & 4.57 & 2.00 & 5.64 & 5.15 & 5.15 & 5.15 & 5.15 \\
\hline $\mathrm{ZrO}_{2}$ & - & - & 3.45 & 3.15 & 3.15 & 3.15 & 3.15 & - & 3.45 & 3.15 & 3.15 & 3.15 & 3.15 \\
\hline $\mathrm{Cl}$ & 0.01 & - & - & - & 0.01 & 0.01 & 0.01 & - & - & - & 0.01 & 0.01 & 0.01 \\
\hline $\mathrm{F}$ & 0.08 & - & - & - & 0.08 & 0.08 & 0.08 & - & - & - & 0.08 & 0.08 & 0.08 \\
\hline $\mathrm{P}_{2} \mathrm{O}_{5}$ & 0.05 & - & - & - & 0.05 & 0.05 & 0.05 & - & - & - & 0.05 & 0.05 & 0.05 \\
\hline $\mathrm{SO}_{3}$ & 0.78 & - & - & - & 0.78 & 4.50 & 0.00 & - & - & - & 0.78 & 4.50 & 0.00 \\
\hline $\mathrm{Re}_{2} \mathrm{O}_{7}$ & - & - & - & - & 0.10 & 0.10 & 0.10 & - & - & - & 0.10 & 0.10 & 0.10 \\
\hline SUM & 8.67 & 0.00 & 100.00 & 91.33 & 100.10 & 103.82 & 99.32 & 0.00 & 100.00 & 91.33 & 100.10 & 103.82 & 99.32 \\
\hline
\end{tabular}


Table 3.10. Composition of Glasses Tested for LAW AZ-101 Per TFCOUP Rev. 2 - High Waste Loading (cont'd).

\begin{tabular}{|c|c|c|c|c|c|c|c|c|c|c|c|c|c|}
\hline \multirow{2}{*}{$\begin{array}{l}\text { Glass } \\
\text { Oxides } \\
\text { Loading } \\
\end{array}$} & \multirow{2}{*}{\begin{tabular}{|c|}
$\mathrm{LAW}$ \\
$\mathrm{AZ}-101$ \\
$+\begin{array}{c}\text { recycled } \\
\mathrm{SO}_{3}\end{array}$ \\
$8.67 \%$ \\
\end{tabular}} & \multirow{2}{*}{ 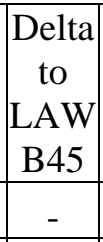 } & \multicolumn{2}{|c|}{$\begin{array}{c}\text { Glass Former } \\
\text { Additives for } \\
\text { LAWB71 }\end{array}$} & \multirow{2}{*}{$\begin{array}{c}\begin{array}{c}\text { LAWB71 } \\
\text { (target) }\end{array} \\
8.67 \%\end{array}$} & \multirow{2}{*}{\begin{tabular}{|c|}
$\begin{array}{c}\text { AAWB71S4 } \\
\text { (target) }\end{array}$ \\
$8.67 \%$ \\
\end{tabular}} & \multirow{2}{*}{\begin{tabular}{|c|}
$\begin{array}{c}\text { LAWB71S0 } \\
\text { (target) }\end{array}$ \\
$8.67 \%$ \\
\end{tabular}} & \multirow{2}{*}{ 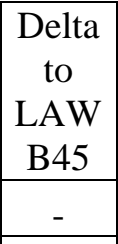 } & \multicolumn{2}{|c|}{$\begin{array}{c}\text { Glass Former } \\
\text { Additives for } \\
\text { LAWB72 }\end{array}$} & \multirow{2}{*}{\begin{tabular}{|c}
$\begin{array}{c}\text { LAWB72 } \\
\text { (target) }\end{array}$ \\
$8.67 \%$ \\
\end{tabular}} & \multirow{2}{*}{\begin{tabular}{|c|}
$\begin{array}{c}\text { LAWB72S4 } \\
\text { (target) }\end{array}$ \\
$8.67 \%$ \\
\end{tabular}} & \multirow{2}{*}{\begin{tabular}{|}
$\begin{array}{c}\text { A AWB72S0 } \\
\text { (target) }\end{array}$ \\
$8.67 \%$
\end{tabular}} \\
\hline & & & $100 \%$ & $91.33 \%$ & & & & & $100 \%$ & $91.33 \%$ & & & \\
\hline $\mathrm{Al}_{2} \mathrm{O}_{3}$ & 0.85 & - & 5.80 & 5.30 & 6.15 & 6.15 & 6.15 & - & 5.80 & 5.30 & 6.15 & 6.15 & 6.15 \\
\hline $\mathrm{B}_{2} \mathrm{O}_{3}$ & 0.00 & -1.55 & 11.80 & 10.78 & 10.78 & 10.78 & 10.78 & - & 13.50 & 12.33 & 12.33 & 12.33 & 12.33 \\
\hline $\mathrm{CaO}$ & 0.00 & - & 7.25 & 6.62 & 6.62 & 6.62 & 6.62 & 0.50 & 7.80 & 7.12 & 7.12 & 7.12 & 7.12 \\
\hline $\mathrm{Cr}_{2} \mathrm{O}_{3}$ & 0.05 & - & - & - & 0.05 & 0.05 & 0.05 & - & - & - & 0.05 & 0.05 & 0.05 \\
\hline $\mathrm{Fe}_{2} \mathrm{O}_{3}$ & 0.00 & -2.00 & 3.56 & 3.25 & 3.25 & 3.25 & 3.25 & -2.00 & 3.56 & 3.25 & 3.25 & 3.25 & 3.25 \\
\hline $\mathrm{K}_{2} \mathrm{O}$ & 0.23 & - & - & - & 0.23 & 0.23 & 0.23 & - & - & - & 0.23 & 0.23 & 0.23 \\
\hline $\mathrm{Li}_{2} \mathrm{O}$ & - & - & 5.05 & 4.61 & 4.61 & 4.61 & 4.61 & -0.50 & 4.50 & 4.11 & 4.11 & 4.11 & 4.11 \\
\hline MgO & - & - & 3.25 & 2.97 & 2.97 & 2.97 & 2.97 & - & 3.25 & 2.97 & 2.97 & 2.97 & 2.97 \\
\hline $\mathrm{Na}_{2} \mathrm{O}$ & 6.62 & - & - & - & 6.62 & 6.62 & 6.62 & - & - & - & 6.62 & 6.62 & 6.62 \\
\hline $\mathrm{SiO}_{2}$ & - & - & 52.50 & 47.95 & 47.95 & 47.95 & 47.95 & - & 52.50 & 47.95 & 47.95 & 47.95 & 47.95 \\
\hline $\mathrm{TiO}_{2}$ & - & 1.55 & 1.70 & 1.55 & 1.55 & 1.55 & 1.55 & - & 0.00 & 0.00 & 0.00 & 0.00 & 0.00 \\
\hline ZnO & - & 2.00 & 5.64 & 5.15 & 5.15 & 5.15 & 5.15 & 2.00 & 5.64 & 5.15 & 5.15 & 5.15 & 5.15 \\
\hline $\mathrm{ZrO}_{2}$ & - & - & 3.45 & 3.15 & 3.15 & 3.15 & 3.15 & - & 3.45 & 3.15 & 3.15 & 3.15 & 3.15 \\
\hline $\mathrm{Cl}$ & 0.01 & - & - & - & 0.01 & 0.01 & 0.01 & - & - & - & 0.01 & 0.01 & 0.01 \\
\hline $\mathrm{F}$ & 0.08 & - & - & - & 0.08 & 0.08 & 0.08 & - & - & - & 0.08 & 0.08 & 0.08 \\
\hline $\mathrm{P}_{2} \mathrm{O}_{5}$ & 0.05 & - & - & - & 0.05 & 0.05 & 0.05 & - & - & - & 0.05 & 0.05 & 0.05 \\
\hline $\mathrm{SO}_{3}$ & 0.78 & - & - & - & 0.78 & 4.50 & 0.00 & - & - & - & 0.78 & 4.50 & 0.00 \\
\hline $\mathrm{Re}_{2} \mathrm{O}_{7}$ & - & - & - & - & 0.10 & 0.10 & 0.10 & - & - & - & 0.10 & 0.10 & 0.10 \\
\hline SUM & 8.67 & 0.00 & 100.00 & 91.33 & 100.10 & 103.82 & 99.32 & 0.00 & 100.00 & 91.33 & 100.10 & 103.82 & 99.32 \\
\hline
\end{tabular}


Table 3.10. Composition of Glasses Tested for LAW AZ-101 Per TFCOUP Rev. 2 - High Waste Loading (cont'd).

\begin{tabular}{|c|c|c|c|c|c|c|c|c|c|c|c|c|c|}
\hline \multirow{2}{*}{\begin{tabular}{|l} 
Glass \\
Oxides \\
Loading \\
\end{tabular}} & \multirow{2}{*}{$\begin{array}{c}\mathrm{LAW} \\
\mathrm{AZ}-101 \\
+\begin{array}{c}\text { recycled } \\
\mathrm{SO}_{3}\end{array} \\
8.67 \% \\
\end{array}$} & \multirow{2}{*}{ 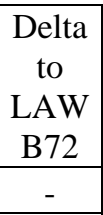 } & \multicolumn{2}{|c|}{$\begin{array}{c}\text { Glass Former } \\
\text { Additives for } \\
\text { LAWB77 }\end{array}$} & \multirow{2}{*}{$\begin{array}{c}\begin{array}{c}\text { LAWB77 } \\
\text { (target) }\end{array} \\
8.67 \% \\
\end{array}$} & \multirow{2}{*}{\begin{tabular}{|c|}
$\begin{array}{c}\text { LAWB77S4 } \\
\text { (target) }\end{array}$ \\
$8.67 \%$ \\
\end{tabular}} & \multirow{2}{*}{\begin{tabular}{|c|}
$\begin{array}{c}\text { LAWB77S0 } \\
\text { (target) }\end{array}$ \\
$8.67 \%$ \\
\end{tabular}} & \multirow{2}{*}{\begin{tabular}{|c|} 
Delta \\
to \\
LAW \\
B72 \\
- \\
\end{tabular}} & \multicolumn{2}{|c|}{$\begin{array}{l}\text { Glass Former } \\
\text { Additives for } \\
\text { LAWB78 }\end{array}$} & \multirow{2}{*}{$\begin{array}{c}\begin{array}{c}\text { LAWB78 } \\
\text { (target) }\end{array} \\
8.67 \% \\
\end{array}$} & \multirow{2}{*}{\begin{tabular}{|c|}
$\begin{array}{c}\text { LAWB78S4 } \\
\text { (target) }\end{array}$ \\
$8.67 \%$
\end{tabular}} & \multirow{2}{*}{\begin{tabular}{|c|}
$\begin{array}{c}\text { LAWB78S0 } \\
\text { (target) }\end{array}$ \\
$8.67 \%$ \\
\end{tabular}} \\
\hline & & & - & $91.33 \%$ & & & & & - & $91.33 \%$ & & & \\
\hline $\mathrm{Al}_{2} \mathrm{O}_{3}$ & 0.85 & - & 5.80 & 5.30 & 6.15 & 6.15 & 6.15 & - & 5.80 & 5.30 & 6.15 & 6.15 & 6.15 \\
\hline $\mathrm{B}_{2} \mathrm{O}_{3}$ & 0.00 & - & 13.50 & 12.33 & 12.33 & 12.33 & 12.33 & - & 13.50 & 12.33 & 12.33 & 12.33 & 12.33 \\
\hline $\mathrm{CaO}$ & 0.00 & -0.50 & 7.25 & 6.62 & 6.62 & 6.62 & 6.62 & - & 7.80 & 7.12 & 7.12 & 7.12 & 7.12 \\
\hline $\mathrm{Cr}_{2} \mathrm{O}_{3}$ & 0.05 & - & - & - & 0.05 & 0.05 & 0.05 & - & - & - & 0.05 & 0.05 & 0.05 \\
\hline $\mathrm{Fe}_{2} \mathrm{O}_{3}$ & 0.00 & \begin{tabular}{|l|}
-1.05 \\
\end{tabular} & 2.41 & 2.20 & 2.20 & 2.20 & 2.20 & - & 3.56 & 3.25 & 3.25 & 3.25 & 3.25 \\
\hline $\mathrm{K}_{2} \mathrm{O}$ & 0.23 & - & - & - & 0.23 & 0.23 & 0.23 & - & - & - & 0.23 & 0.23 & 0.23 \\
\hline $\mathrm{Li}_{2} \mathrm{O}$ & - & - & 4.50 & 4.11 & 4.11 & 4.11 & 4.11 & -1.06 & 3.34 & 3.05 & 3.05 & 3.05 & 3.05 \\
\hline $\mathrm{MgO}$ & - & - & 3.25 & 2.97 & 2.97 & 2.97 & 2.97 & - & 3.25 & 2.97 & 2.97 & 2.97 & 2.97 \\
\hline $\mathrm{Na}_{2} \mathrm{O}$ & 6.62 & - & - & - & 6.62 & 6.62 & 6.62 & 3.16 & 3.46 & 3.16 & 9.78 & 9.78 & 9.78 \\
\hline $\mathrm{SiO}_{2}$ & - & - & 52.50 & 47.95 & 47.95 & 47.95 & 47.95 & -0.95 & 51.46 & 47.00 & 47.00 & 47.00 & 47.00 \\
\hline $\mathrm{TiO}_{2}$ & - & 1.55 & 1.70 & 1.55 & 1.55 & 1.55 & 1.55 & - & 0.00 & 0.00 & 0.00 & 0.00 & 0.00 \\
\hline $\mathrm{ZnO}$ & - & - & 5.64 & 5.15 & 5.15 & 5.15 & 5.15 & -1.15 & 4.38 & 4.00 & 4.00 & 4.00 & 4.00 \\
\hline $\mathrm{ZrO}_{2}$ & - & - & 3.45 & 3.15 & 3.15 & 3.15 & 3.15 & - & 3.45 & 3.15 & 3.15 & 3.15 & 3.15 \\
\hline $\mathrm{Cl}$ & 0.01 & - & - & - & 0.01 & 0.01 & 0.01 & - & - & - & 0.01 & 0.01 & 0.01 \\
\hline$F$ & 0.08 & - & - & - & 0.08 & 0.08 & 0.08 & - & - & - & 0.08 & 0.08 & 0.08 \\
\hline $\mathrm{P}_{2} \mathrm{O}_{5}$ & 0.05 & - & - & - & 0.05 & 0.05 & 0.05 & - & - & - & 0.05 & 0.05 & 0.05 \\
\hline $\mathrm{SO}_{3}$ & 0.78 & - & - & - & 0.78 & 4.50 & 0.00 & - & - & - & 0.78 & 4.50 & 0.00 \\
\hline $\mathrm{Re}_{2} \mathrm{O}_{7}$ & - & - & - & - & 0.10 & 0.10 & 0.10 & - & - & - & 0.10 & 0.10 & 0.10 \\
\hline SUM & 8.67 & 0.00 & 100.00 & 91.33 & 100.10 & 103.82 & 99.32 & 0.00 & 100.00 & 91.33 & 100.10 & 103.82 & 99.32 \\
\hline
\end{tabular}


Table 3.10. Composition of Glasses Tested for LAW AZ-101 Per TFCOUP Rev. 2 - High Waste Loading (cont'd).

\begin{tabular}{|c|c|c|c|c|c|c|c|c|c|c|c|c|c|}
\hline \multirow{2}{*}{\begin{tabular}{|l} 
Glass \\
Oxides \\
Loading \\
\end{tabular}} & \multirow{2}{*}{$\begin{array}{c}\text { LAW } \\
\text { AZ-101 } \\
+ \text { recycled } \\
\mathrm{SO}_{3} \\
8.67 \%\end{array}$} & \multirow{2}{*}{ 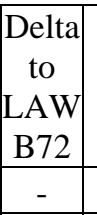 } & \multicolumn{2}{|c|}{$\begin{array}{l}\text { Glass Former } \\
\text { Additives for } \\
\text { LAWB79 }\end{array}$} & \multirow{2}{*}{$\begin{array}{c}\begin{array}{c}\text { LAWB79 } \\
\text { (target) }\end{array} \\
8.67 \% \\
\end{array}$} & \multirow{2}{*}{\begin{tabular}{|c|}
$\begin{array}{c}\text { LAWB79S4 } \\
\text { (target) }\end{array}$ \\
$8.67 \%$ \\
\end{tabular}} & \multirow{2}{*}{\begin{tabular}{|c|}
$\begin{array}{c}\text { LAWB79S0 } \\
\text { (target) }\end{array}$ \\
$8.67 \%$ \\
\end{tabular}} & \multirow{2}{*}{\begin{tabular}{|c|}
$\begin{array}{c}\text { Delta } \\
\text { to } \\
\text { B72 }\end{array}$ \\
- \\
\end{tabular}} & \multicolumn{2}{|c|}{$\begin{array}{l}\text { Glass Former } \\
\text { Additives for } \\
\text { LAWB80 }\end{array}$} & \multirow{2}{*}{$\begin{array}{c}\begin{array}{c}\text { LAWB80 } \\
\text { (target) }\end{array} \\
8.67 \% \\
\end{array}$} & \multirow{2}{*}{\begin{tabular}{|c|}
$\begin{array}{c}\text { LAWB80S4 } \\
\text { (target) }\end{array}$ \\
$8.67 \%$ \\
\end{tabular}} & \multirow{2}{*}{\begin{tabular}{|c}
$\begin{array}{c}\text { LAWB80S0 } \\
\text { (target) }\end{array}$ \\
$8.67 \%$ \\
\end{tabular}} \\
\hline & & & - & $91.33 \%$ & & & & & - & $91.33 \%$ & & & \\
\hline $\mathrm{Al}_{2} \mathrm{O}_{3}$ & 0.85 & - & 5.80 & 5.30 & 6.15 & 6.15 & 6.15 & - & 5.80 & 5.30 & 6.15 & 6.15 & 6.15 \\
\hline $\mathrm{B}_{2} \mathrm{O}_{3}$ & 0.00 & - & 13.50 & 12.33 & 12.33 & 12.33 & 12.33 & - & 13.50 & 12.33 & 12.33 & 12.33 & 12.33 \\
\hline $\mathrm{CaO}$ & 0.00 & 0.00 & 7.80 & 7.12 & 7.12 & 7.12 & 7.12 & 0.00 & 7.80 & 7.12 & 7.12 & 7.12 & 7.12 \\
\hline $\mathrm{Cr}_{2} \mathrm{O}_{3}$ & 0.05 & - & - & - & 0.05 & 0.05 & 0.05 & - & - & - & 0.05 & 0.05 & 0.05 \\
\hline $\mathrm{Fe}_{2} \mathrm{O}_{3}$ & 0.00 & 0.00 & 3.56 & 3.25 & 3.25 & 3.25 & 3.25 & 0.00 & 3.56 & 3.25 & 3.25 & 3.25 & 3.25 \\
\hline $\mathrm{K}_{2} \mathrm{O}$ & 0.23 & 0.00 & - & - & 0.23 & 0.23 & 0.23 & 1.75 & 1.92 & 1.75 & 1.99 & 1.99 & 1.99 \\
\hline $\mathrm{Li}_{2} \mathrm{O}$ & - & -0.60 & 3.84 & 3.51 & 3.51 & 3.51 & 3.51 & -0.60 & 3.84 & 3.51 & 3.51 & 3.51 & 3.51 \\
\hline $\mathrm{MgO}$ & - & - & 3.25 & 2.97 & 2.97 & 2.97 & 2.97 & - & 3.25 & 2.97 & 2.97 & 2.97 & 2.97 \\
\hline $\mathrm{Na}_{2} \mathrm{O}$ & 6.62 & 2.00 & 2.19 & 2.00 & 8.62 & 8.62 & 8.62 & 0.00 & 0.00 & 0.00 & 6.62 & 6.62 & 6.62 \\
\hline $\mathrm{SiO}_{2}$ & - & -0.25 & 52.23 & 47.70 & 47.70 & 47.70 & 47.70 & 0.00 & 52.50 & 47.95 & 47.95 & 47.95 & 47.95 \\
\hline $\mathrm{TiO}_{2}$ & - & & 0.00 & 0.00 & 0.00 & 0.00 & 0.00 & 0.00 & 0.00 & 0.00 & 0.00 & 0.00 & 0.00 \\
\hline $\mathrm{ZnO}$ & - & -1.15 & 4.38 & 4.00 & 4.00 & 4.00 & 4.00 & -1.15 & 4.38 & 4.00 & 4.00 & 4.00 & 4.00 \\
\hline $\mathrm{ZrO}_{2}$ & - & - & 3.45 & 3.15 & 3.15 & 3.15 & 3.15 & - & 3.45 & 3.15 & 3.15 & 3.15 & 3.15 \\
\hline $\mathrm{Cl}$ & 0.01 & - & - & - & 0.01 & 0.01 & 0.01 & - & - & - & 0.01 & 0.01 & 0.01 \\
\hline$F$ & 0.08 & - & - & - & 0.08 & 0.08 & 0.08 & - & - & - & 0.08 & 0.08 & 0.08 \\
\hline $\mathrm{P}_{2} \mathrm{O}_{5}$ & 0.05 & - & - & - & 0.05 & 0.05 & 0.05 & - & - & - & 0.05 & 0.05 & 0.05 \\
\hline $\mathrm{SO}_{3}$ & 0.78 & - & - & - & 0.78 & 4.50 & 0.00 & - & - & - & 0.78 & 4.50 & 0.00 \\
\hline $\mathrm{Re}_{2} \mathrm{O}_{7}$ & - & - & - & - & 0.10 & 0.10 & 0.10 & - & - & - & 0.10 & 0.10 & 0.10 \\
\hline SUM & 8.67 & 0.00 & 100.00 & 91.33 & 100.10 & 103.82 & 99.32 & 0.00 & 100.00 & 91.33 & 100.10 & 103.82 & 99.32 \\
\hline
\end{tabular}


Table 3.10. Composition of Glasses Tested for LAW AZ-101 Per TFCOUP Rev. 2 - High Waste Loading (cont'd).

\begin{tabular}{|c|c|c|c|c|c|c|c|c|c|c|c|}
\hline $\begin{array}{l}\text { Glass } \\
\text { Oxides }\end{array}$ & $\begin{array}{l}\text { LAW AZ-101 } \\
+ \text { recycled } \mathrm{SO}_{3}\end{array}$ & $\begin{array}{c}\text { Delta to } \\
\text { LAW } \\
\text { B72 }\end{array}$ & $\begin{array}{r}\text { Glass Forr } \\
\mathrm{L}\end{array}$ & $\begin{array}{l}\text { Idditives for } \\
381\end{array}$ & $\begin{array}{l}\text { LAWB81 } \\
\text { (target) }\end{array}$ & $\begin{array}{c}\text { LAWB81S4 } \\
\text { (target) }\end{array}$ & $\begin{array}{c}\text { Delta to } \\
\text { LAW } \\
\text { B7? }\end{array}$ & $\begin{array}{r}\text { Glass Fo } \\
\text { for }\end{array}$ & $\begin{array}{l}\text { Additives } \\
\text { JB82 }\end{array}$ & $\begin{array}{c}\text { LAWB82 } \\
\text { (target) }\end{array}$ & $\begin{array}{l}\text { LAWB82S4 } \\
\text { (target) }\end{array}$ \\
\hline Loading & $8.67 \%$ & - & $100 \%$ & $91.33 \%$ & $8.67 \%$ & $8.67 \%$ & - & $100 \%$ & $91.33 \%$ & $8.67 \%$ & $8.67 \%$ \\
\hline $\mathrm{Al}_{2} \mathrm{O}_{3}$ & 0.85 & - & 5.80 & 5.30 & 6.15 & 6.15 & - & 5.80 & 5.30 & 6.15 & 6.15 \\
\hline $\mathrm{B}_{2} \mathrm{O}_{3}$ & 0.00 & - & 13.50 & 12.33 & 12.33 & 12.33 & -2.25 & 11.04 & 10.08 & 10.08 & 10.08 \\
\hline $\mathrm{CaO}$ & 0.00 & - & 7.80 & 7.12 & 7.12 & 7.12 & - & 7.80 & 7.12 & 7.12 & 7.12 \\
\hline $\mathrm{Cr}_{2} \mathrm{O}_{3}$ & 0.05 & - & - & - & 0.05 & 0.05 & - & - & - & 0.05 & 0.05 \\
\hline $\mathrm{Fe}_{2} \mathrm{O}_{3}$ & 0.00 & - & 3.56 & 3.25 & 3.25 & 3.25 & 6.25 & 10.40 & 9.50 & 9.50 & 9.50 \\
\hline $\mathrm{K}_{2} \mathrm{O}$ & 0.23 & - & - & 0.00 & 0.23 & 0.23 & - & - & 0.00 & 0.23 & 0.23 \\
\hline $\mathrm{Li}_{2} \mathrm{O}$ & - & 0.15 & 4.67 & 4.26 & 4.26 & 4.26 & 0.15 & 4.67 & 4.26 & 4.26 & 4.26 \\
\hline $\mathrm{MgO}$ & - & - & 3.25 & 2.97 & 2.97 & 2.97 & -1.49 & 1.62 & 1.48 & 1.48 & 1.48 \\
\hline $\mathrm{Na}_{2} \mathrm{O}$ & 6.62 & - & - & 0.00 & 6.62 & 6.62 & - & - & 0.00 & 6.62 & 6.62 \\
\hline $\mathrm{SiO}_{2}$ & - & - & 52.50 & 47.95 & 47.95 & 47.95 & -2.51 & 49.75 & 45.44 & 45.44 & 45.44 \\
\hline $\mathrm{TiO}_{2}$ & - & - & 0.00 & 0.00 & 0.00 & 0.00 & - & 0.00 & 0.00 & 0.00 & 0.00 \\
\hline $\mathrm{ZnO}$ & - & -0.15 & 5.48 & 5.00 & 5.00 & 5.00 & -0.15 & 5.48 & 5.00 & 5.00 & 5.00 \\
\hline $\mathrm{ZrO}_{2}$ & - & - & 3.45 & 3.15 & 3.15 & 3.15 & - & 3.45 & 3.15 & 3.15 & 3.15 \\
\hline $\mathrm{Cl}$ & 0.01 & - & - & - & 0.01 & 0.01 & - & - & - & 0.01 & 0.01 \\
\hline $\mathrm{F}$ & 0.08 & - & - & - & 0.08 & 0.08 & - & - & - & 0.08 & 0.08 \\
\hline $\mathrm{P}_{2} \mathrm{O}_{5}$ & 0.05 & - & - & - & 0.05 & 0.05 & - & - & - & 0.05 & 0.05 \\
\hline $\mathrm{SO}_{3}$ & 0.78 & - & - & - & 0.78 & 4.50 & - & - & - & 0.78 & 4.50 \\
\hline $\mathrm{Re}_{2} \mathrm{O}_{7}$ & - & - & - & - & 0.10 & 0.10 & - & - & - & 0.10 & 0.10 \\
\hline SUM & 8.67 & 0.00 & 100.00 & 91.33 & 100.10 & 103.82 & 0.00 & 100.00 & 91.33 & 100.10 & 103.82 \\
\hline
\end{tabular}


Table 3.11. Composition of Glasses Tested for LAW AZ-101 Per TFCOUP Rev. 2 - Low Waste Loading.

\begin{tabular}{|c|c|c|c|c|c|c|c|c|c|c|c|}
\hline \multirow{2}{*}{\begin{tabular}{|l} 
Glass \\
Oxides \\
Loading \\
\end{tabular}} & \multirow{2}{*}{$\begin{array}{c}\text { Pretreated } \\
\text { LAW } \\
\text { AZ-101 } \\
+ \text { recycled } \\
\mathrm{SO}_{3} \\
7.17 \% \\
\end{array}$} & \multicolumn{2}{|c|}{$\begin{array}{c}\text { Glass Former Additives } \\
\text { for LAWB83 }\end{array}$} & \multirow{2}{*}{$\begin{array}{c}\begin{array}{c}\text { LAWB83 } \\
\text { (target) }\end{array} \\
7.17 \% \\
\end{array}$} & \multirow{2}{*}{$\begin{array}{l}\text { LAWB83S4 } \\
\text { (target) } \\
\\
7.17 \%\end{array}$} & \multirow{2}{*}{$\begin{array}{l}\text { LAWB83S0 } \\
\text { (target) } \\
\\
7.17 \%\end{array}$} & \multirow{2}{*}{$\begin{array}{c}\text { Delta } \\
\text { to } \\
\text { LAW } \\
\text { B83 } \\
- \\
\end{array}$} & \multicolumn{2}{|c|}{$\begin{array}{c}\text { Glass Former } \\
\text { Additives for } \\
\text { LAWB84 }\end{array}$} & \multirow{2}{*}{$\begin{array}{c}\begin{array}{c}\text { LAWB84 } \\
\text { (target) }\end{array} \\
7.17 \% \\
\end{array}$} & \multirow{2}{*}{$\begin{array}{c}\begin{array}{c}\text { LAWB84S4 } \\
\text { (target) }\end{array} \\
7.17 \%\end{array}$} \\
\hline & & $100 \%$ & $92.83 \%$ & & & & & $100 \%$ & $92.83 \%$ & & \\
\hline $\mathrm{Al}_{2} \mathrm{O}_{3}$ & 0.71 & 5.90 & 5.48 & 6.18 & 6.18 & 6.18 & - & 5.90 & 5.48 & 6.18 & 6.18 \\
\hline $\mathrm{B}_{2} \mathrm{O}_{3}$ & 0.00 & 10.80 & 10.03 & 10.03 & 10.03 & 10.03 & - & 10.80 & 10.03 & 10.03 & 10.03 \\
\hline $\mathrm{CaO}$ & 0.00 & 7.30 & 6.78 & 6.78 & 6.78 & 6.78 & -0.10 & 7.20 & 6.68 & 6.68 & 6.68 \\
\hline $\mathrm{Cr}_{2} \mathrm{O}_{3}$ & 0.04 & - & - & 0.04 & 0.04 & 0.04 & - & - & - & 0.04 & 0.04 \\
\hline $\mathrm{Fe}_{2} \mathrm{O}_{3}$ & 0.00 & 5.70 & 5.29 & 5.29 & 5.29 & 5.29 & - & 5.70 & 5.29 & 5.29 & 5.29 \\
\hline $\mathrm{K}_{2} \mathrm{O}$ & 0.19 & - & - & 0.19 & 0.19 & 0.19 & - & - & - & 0.19 & 0.19 \\
\hline $\mathrm{Li}_{2} \mathrm{O}$ & - & 4.64 & 4.31 & 4.31 & 4.31 & 4.31 & 0.10 & 4.74 & 4.40 & 4.40 & 4.40 \\
\hline $\mathrm{MgO}$ & - & 3.20 & 2.97 & 2.97 & 2.97 & 2.97 & - & 3.20 & 2.97 & 2.97 & 2.97 \\
\hline $\mathrm{Na}_{2} \mathrm{O}$ & 5.47 & - & - & 5.47 & 5.47 & 5.47 & - & - & - & 5.47 & 5.47 \\
\hline $\mathrm{SiO}_{2}$ & - & 52.35 & 48.60 & 48.60 & 48.60 & 48.60 & - & 52.35 & 48.60 & 48.60 & 48.60 \\
\hline $\mathrm{TiO}_{2}$ & - & 1.50 & 1.39 & 1.39 & 1.39 & 1.39 & - & 1.50 & 1.39 & 1.39 & 1.39 \\
\hline $\mathrm{ZnO}$ & - & 5.21 & 4.84 & 4.84 & 4.84 & 4.84 & - & 5.21 & 4.84 & 4.84 & 4.84 \\
\hline $\mathrm{ZrO}_{2}$ & - & 3.40 & 3.16 & 3.16 & 3.16 & 3.16 & - & 3.40 & 3.16 & 3.16 & 3.16 \\
\hline $\mathrm{Cl}$ & 0.01 & - & - & 0.01 & 0.01 & 0.01 & - & - & - & 0.01 & 0.01 \\
\hline $\mathrm{F}$ & 0.06 & - & - & 0.06 & 0.06 & 0.06 & - & - & - & 0.06 & 0.06 \\
\hline $\mathrm{P}_{2} \mathrm{O}_{5}$ & 0.04 & - & - & 0.04 & 0.04 & 0.04 & - & - & - & 0.04 & 0.04 \\
\hline $\mathrm{SO}_{3}$ & 0.65 & - & - & 0.65 & 4.50 & 0.00 & - & - & - & 0.65 & 4.50 \\
\hline $\mathrm{Re}_{2} \mathrm{O}_{7}$ & - & - & - & 0.10 & 0.10 & 0.10 & - & - & - & 0.10 & 0.10 \\
\hline SUM & 7.17 & 100.00 & 92.83 & 100.10 & 103.95 & 99.45 & 0.00 & 100.00 & 92.83 & 100.10 & 103.95 \\
\hline
\end{tabular}


Table 3.11. Composition of Glasses Tested for LAW AZ-101 Per TFCOUP Rev. 2 - Low Waste Loading (cont'd).

\begin{tabular}{|c|c|c|c|c|c|c|c|c|c|c|c|}
\hline \multirow{2}{*}{$\begin{array}{l}\text { Glass } \\
\text { Oxides } \\
\text { Loading }\end{array}$} & \multirow{2}{*}{$\begin{array}{c}\text { Pretreated } \\
\text { LAW } \\
\text { AZ-101 } \\
+ \text { recycled } \\
\mathrm{SO}_{3} \\
7.17 \% \\
\end{array}$} & \multirow{2}{*}{$\begin{array}{c}\text { Delta } \\
\text { to } \\
\text { LAW } \\
\text { B83 } \\
- \\
\end{array}$} & \multicolumn{2}{|c|}{$\begin{array}{l}\text { Glass Former } \\
\text { Additives for } \\
\text { LAWB85 }\end{array}$} & \multirow{2}{*}{$\begin{array}{c}\begin{array}{c}\text { LAWB85 } \\
\text { (target) }\end{array} \\
7.17 \%\end{array}$} & \multirow{2}{*}{$\begin{array}{c}\text { LAWB85S4 } \\
\text { (target) } \\
7.17 \%\end{array}$} & \multirow{2}{*}{$\begin{array}{l}\text { Delta } \\
\text { to } \\
\text { LAW } \\
\text { B83 } \\
- \\
\end{array}$} & \multicolumn{2}{|c|}{$\begin{array}{l}\text { Glass Former } \\
\text { Additives for } \\
\text { LAWB86 }\end{array}$} & \multirow{2}{*}{$\begin{array}{c}\begin{array}{c}\text { LAWB86 } \\
\text { (target) }\end{array} \\
7.17 \%\end{array}$} & \multirow{2}{*}{$\begin{array}{c}\text { LAWB86S4 } \\
\text { (target) } \\
\\
7.17 \%\end{array}$} \\
\hline & & & $100 \%$ & $92.83 \%$ & & & & $100 \%$ & $92.83 \%$ & & \\
\hline $\mathrm{Al}_{2} \mathrm{O}_{3}$ & 0.71 & - & 5.90 & 5.48 & 6.18 & 6.18 & - & 5.90 & 5.48 & 6.18 & 6.18 \\
\hline $\mathrm{B}_{2} \mathrm{O}_{3}$ & 0.00 & 1.49 & 12.41 & 11.52 & 11.52 & 11.52 & 2.39 & 13.37 & 12.41 & 12.41 & 12.41 \\
\hline $\mathrm{CaO}$ & 0.00 & -1.49 & 5.69 & 5.28 & 5.28 & 5.28 & -1.04 & 6.18 & 5.73 & 5.73 & 5.73 \\
\hline $\mathrm{Cr}_{2} \mathrm{O}_{3}$ & 0.04 & - & - & - & 0.04 & 0.04 & - & - & - & 0.04 & 0.04 \\
\hline $\mathrm{Fe}_{2} \mathrm{O}_{3}$ & 0.00 & - & 5.70 & 5.29 & 5.29 & 5.29 & - & 5.70 & 5.29 & 5.29 & 5.29 \\
\hline $\mathrm{K}_{2} \mathrm{O}$ & 0.19 & - & - & - & 0.19 & 0.19 & - & - & - & 0.19 & 0.19 \\
\hline $\mathrm{Li}_{2} \mathrm{O}$ & - & - & 4.64 & 4.31 & 4.31 & 4.31 & 0.05 & 4.69 & 4.35 & 4.35 & 4.35 \\
\hline $\mathrm{MgO}$ & - & - & 3.20 & 2.97 & 2.97 & 2.97 & - & 3.20 & 2.97 & 2.97 & 2.97 \\
\hline $\mathrm{Na}_{2} \mathrm{O}$ & 5.47 & - & - & - & 5.47 & 5.47 & - & - & - & 5.47 & 5.47 \\
\hline $\mathrm{SiO}_{2}$ & - & - & 52.35 & 48.60 & 48.60 & 48.60 & - & \begin{tabular}{|l|}
52.35 \\
\end{tabular} & 48.60 & 48.60 & 48.60 \\
\hline $\mathrm{TiO}_{2}$ & - & - & 1.50 & 1.39 & 1.39 & 1.39 & -1.39 & 0.00 & 0.00 & 0.00 & 0.00 \\
\hline $\mathrm{ZnO}$ & - & - & 5.21 & 4.84 & 4.84 & 4.84 & - & 5.21 & 4.84 & 4.84 & 4.84 \\
\hline $\mathrm{ZrO}_{2}$ & - & - & 3.40 & 3.16 & 3.16 & 3.16 & - & 3.40 & 3.16 & 3.16 & 3.16 \\
\hline $\mathrm{Cl}$ & 0.01 & - & - & - & 0.01 & 0.01 & - & - & - & 0.01 & 0.01 \\
\hline$F$ & 0.06 & - & - & - & 0.06 & 0.06 & - & - & - & 0.06 & 0.06 \\
\hline $\mathrm{P}_{2} \mathrm{O}_{5}$ & 0.04 & - & - & - & 0.04 & 0.04 & - & - & - & 0.04 & 0.04 \\
\hline $\mathrm{SO}_{3}$ & 0.65 & - & - & - & 0.65 & 4.50 & - & - & - & 0.65 & 4.50 \\
\hline $\mathrm{Re}_{2} \mathrm{O}_{7}$ & - & - & - & - & 0.10 & 0.10 & - & - & - & 0.10 & 0.10 \\
\hline SUM & 7.17 & 0.00 & 100.00 & 92.83 & 100.10 & 103.95 & 0.00 & 100.00 & 92.83 & 100.10 & 103.95 \\
\hline
\end{tabular}


Table 3.12. Composition of Glasses Tested for LAW AZ-101 Per TFCOUP Rev. 2 - Alkali Variation.

\begin{tabular}{|c|c|c|c|c|c|c|c|c|c|c|c|}
\hline \multirow{2}{*}{\begin{tabular}{|l} 
Glass \\
Oxides \\
Loading
\end{tabular}} & \multirow{2}{*}{\begin{tabular}{|c}
$\begin{array}{c}\text { Pretreated } \\
\text { LAW AZ-101 } \\
+ \\
\text { recycled } \\
\mathrm{SO}_{3}\end{array}$ \\
$7.17 \%$
\end{tabular}} & \multirow{2}{*}{\begin{tabular}{|c|} 
Delta to \\
LAWB83 \\
- \\
\end{tabular}} & \multicolumn{2}{|c|}{$\begin{array}{c}\text { Glass Former } \\
\text { Additives for } \\
\text { LAWB89 }\end{array}$} & \multirow{2}{*}{$\begin{array}{c}\begin{array}{c}\text { LAWB89 } \\
\text { (target) }\end{array} \\
7.17 \%\end{array}$} & \multirow{2}{*}{$\begin{array}{c}\begin{array}{c}\text { LAWB89S4 } \\
\text { (target) }\end{array} \\
7.17 \% \\
\end{array}$} & \multirow{2}{*}{\begin{tabular}{|c}
$\begin{array}{c}\text { Delta to } \\
\text { LAW } \\
\text { B83 }\end{array}$ \\
- \\
\end{tabular}} & \multicolumn{2}{|c|}{$\begin{array}{c}\text { Glass Former } \\
\text { Additives for } \\
\text { LAWB90 }\end{array}$} & \multirow{2}{*}{$\begin{array}{c}\begin{array}{c}\text { LAWB90 } \\
\text { (target) }\end{array} \\
7.17 \%\end{array}$} & \multirow{2}{*}{$\begin{array}{c}\begin{array}{c}\text { LAWB90S } \\
\text { (target) }\end{array} \\
7.17 \%\end{array}$} \\
\hline & & & - & $92.83 \%$ & & & & - & $92.83 \%$ & & \\
\hline $\mathrm{Al}_{2} \mathrm{O}_{3}$ & 0.71 & - & 5.90 & 5.48 & 6.18 & 6.18 & - & 5.90 & 5.48 & 6.18 & 6.18 \\
\hline $\mathrm{B}_{2} \mathrm{O}_{3}$ & 0.00 & - & 10.80 & 10.03 & 10.03 & 10.03 & - & 10.80 & 10.03 & 10.03 & 10.03 \\
\hline $\mathrm{CaO}$ & 0.00 & - & 7.30 & 6.78 & 6.78 & 6.78 & - & 7.30 & 6.78 & 6.78 & 6.78 \\
\hline $\mathrm{Cr}_{2} \mathrm{O}_{3}$ & 0.04 & - & - & - & 0.04 & 0.04 & - & - & - & 0.04 & 0.04 \\
\hline $\mathrm{Fe}_{2} \mathrm{O}_{3}$ & 0.00 & - & 5.70 & 5.29 & 5.29 & 5.29 & - & 5.70 & 5.29 & 5.29 & 5.29 \\
\hline $\mathrm{K}_{2} \mathrm{O}$ & 0.19 & - & - & - & 0.19 & 0.19 & - & - & - & 0.19 & 0.19 \\
\hline $\mathrm{Li}_{2} \mathrm{O}$ & - & 0.70 & 5.39 & 5.00 & 5.00 & 5.00 & -0.70 & 3.89 & 3.61 & 3.61 & 3.61 \\
\hline $\mathrm{MgO}$ & - & - & 3.20 & 2.97 & 2.97 & 2.97 & & 3.20 & 2.97 & 2.97 & 2.97 \\
\hline $\mathrm{Na}_{2} \mathrm{O}$ & 5.47 & -1.39 & -1.50 & -1.39 & 4.08 & 4.08 & 1.39 & 1.50 & 1.39 & 6.86 & 6.86 \\
\hline $\mathrm{SiO}_{2}$ & - & 0.70 & 53.10 & 49.29 & 49.29 & 49.29 & -0.70 & 51.60 & 47.90 & 47.90 & 47.90 \\
\hline $\mathrm{TiO}_{2}$ & - & - & 1.50 & 1.39 & 1.39 & 1.39 & - & 1.50 & 1.39 & 1.39 & 1.39 \\
\hline $\mathrm{ZnO}$ & - & - & 5.21 & 4.84 & 4.84 & 4.84 & - & 5.21 & 4.84 & 4.84 & 4.84 \\
\hline $\mathrm{ZrO}_{2}$ & - & - & 3.40 & 3.16 & 3.16 & 3.16 & - & 3.40 & 3.16 & 3.16 & 3.16 \\
\hline $\mathrm{Cl}$ & 0.01 & - & - & - & 0.01 & 0.01 & - & - & - & 0.01 & 0.01 \\
\hline$F$ & 0.06 & - & - & - & 0.06 & 0.06 & - & - & - & 0.06 & 0.06 \\
\hline $\mathrm{P}_{2} \mathrm{O}_{5}$ & 0.04 & - & - & - & 0.04 & 0.04 & - & - & - & 0.04 & 0.04 \\
\hline $\mathrm{SO}_{3}$ & 0.65 & - & - & - & 0.65 & 4.50 & - & - & - & 0.65 & 4.50 \\
\hline $\mathrm{Re}_{2} \mathrm{O}_{7}$ & - & - & - & - & 0.10 & 0.10 & - & - & - & 0.10 & 0.10 \\
\hline SUM & 7.17 & 0.00 & 100.00 & 92.83 & 100.10 & 103.95 & 0.00 & 100.00 & 92.83 & 100.10 & 103.95 \\
\hline
\end{tabular}


Table 3.12. Composition of Glasses Tested for LAW AZ-101 Per TFCOUP Rev. 2 - Alkali Variation (cont'd).

\begin{tabular}{|c|c|c|c|c|c|c|c|c|c|c|c|}
\hline \multirow{2}{*}{\begin{tabular}{|l} 
Glass \\
Oxides \\
Loading \\
\end{tabular}} & \multirow{2}{*}{$\begin{array}{c}\text { Pretreated } \\
\text { LAW } \\
\text { AZ-101 } \\
+ \text { recycled } \\
\mathrm{SO}_{3} \\
7.17 \% \\
\end{array}$} & \multirow{2}{*}{$\begin{array}{c}\text { Delta to } \\
\text { LAWB83 } \\
- \\
\end{array}$} & \multicolumn{2}{|c|}{$\begin{array}{c}\text { Glass Former } \\
\text { Additives for } \\
\text { LAWB91 }\end{array}$} & \multirow{2}{*}{$\begin{array}{c}\begin{array}{c}\text { LAWB91 } \\
\text { (target) }\end{array} \\
7.17 \% \\
\end{array}$} & \multirow{2}{*}{$\begin{array}{c}\text { LAWB91S4 } \\
\text { (target) } \\
7.17 \% \\
\end{array}$} & \multirow{2}{*}{\begin{tabular}{|c|} 
Delta to \\
LAWB83 \\
- \\
\end{tabular}} & \multicolumn{2}{|c|}{$\begin{array}{c}\text { Glass Former } \\
\text { Additives for } \\
\text { LAWB92 }\end{array}$} & \multirow{2}{*}{$\begin{array}{c}\begin{array}{c}\text { LAWB92 } \\
\text { (target) }\end{array} \\
7.17 \% \\
\end{array}$} & \multirow{2}{*}{$\begin{array}{c}\begin{array}{c}\text { LAWB92S4 } \\
\text { (target) }\end{array} \\
7.17 \% \\
\end{array}$} \\
\hline & & & - & $92.83 \%$ & & & & - & $92.83 \%$ & & \\
\hline $\mathrm{Al}_{2} \mathrm{O}_{3}$ & 0.71 & - & 5.90 & 5.48 & 6.18 & 6.18 & - & 5.90 & 5.48 & 6.18 & 6.18 \\
\hline $\mathrm{B}_{2} \mathrm{O}_{3}$ & 0.00 & - & 10.80 & 10.03 & 10.03 & 10.03 & - & 10.80 & 10.03 & 10.03 & 10.03 \\
\hline $\mathrm{CaO}$ & 0.00 & - & 7.30 & 6.78 & 6.78 & 6.78 & - & 7.30 & 6.78 & 6.78 & 6.78 \\
\hline $\mathrm{Cr}_{2} \mathrm{O}_{3}$ & 0.04 & - & - & - & 0.04 & 0.04 & - & - & - & 0.04 & 0.04 \\
\hline $\mathrm{Fe}_{2} \mathrm{O}_{3}$ & 0.00 & - & 5.70 & 5.29 & 5.29 & 5.29 & - & 5.70 & 5.29 & 5.29 & 5.29 \\
\hline $\mathrm{K}_{2} \mathrm{O}$ & 0.19 & - & - & - & 0.19 & 0.19 & - & - & - & 0.19 & 0.19 \\
\hline $\mathrm{Li}_{2} \mathrm{O}$ & - & -1.39 & 3.14 & 2.91 & 2.91 & 2.91 & -2.09 & 2.39 & 2.22 & 2.22 & 2.22 \\
\hline $\mathrm{MgO}$ & - & - & 3.20 & 2.97 & 2.97 & 2.97 & - & 3.20 & 2.97 & 2.97 & 2.97 \\
\hline $\mathrm{Na}_{2} \mathrm{O}$ & 5.47 & 3.25 & 3.50 & 3.25 & 8.72 & 8.72 & 4.64 & 5.00 & 4.64 & 10.11 & 10.11 \\
\hline $\mathrm{SiO}_{2}$ & - & -1.86 & 50.35 & 46.74 & 46.74 & 46.74 & -2.55 & 49.60 & 46.05 & 46.05 & 46.05 \\
\hline $\mathrm{TiO}_{2}$ & - & - & 1.50 & 1.39 & 1.39 & 1.39 & - & 1.50 & 1.39 & 1.39 & 1.39 \\
\hline $\mathrm{ZnO}$ & - & - & 5.21 & 4.84 & 4.84 & 4.84 & - & 5.21 & 4.84 & 4.84 & 4.84 \\
\hline $\mathrm{ZrO}_{2}$ & - & - & 3.40 & 3.16 & 3.16 & 3.16 & - & 3.40 & 3.16 & 3.16 & 3.16 \\
\hline $\mathrm{Cl}$ & 0.01 & - & - & - & 0.01 & 0.01 & - & - & - & 0.01 & 0.01 \\
\hline$F$ & 0.06 & - & - & - & 0.06 & 0.06 & - & - & - & 0.06 & 0.06 \\
\hline $\mathrm{P}_{2} \mathrm{O}_{5}$ & 0.04 & - & - & - & 0.04 & 0.04 & - & - & - & 0.04 & 0.04 \\
\hline $\mathrm{SO}_{3}$ & 0.65 & - & - & - & 0.65 & 4.50 & - & - & - & 0.65 & 4.50 \\
\hline $\mathrm{Re}_{2} \mathrm{O}_{7}$ & - & - & - & - & 0.10 & 0.10 & - & - & - & 0.10 & 0.10 \\
\hline SUM & 7.17 & 0.00 & 100.00 & 92.83 & 100.10 & 103.95 & 0.00 & 100.00 & 92.83 & 100.10 & 103.95 \\
\hline
\end{tabular}


Table 3.12. Composition of Glasses Tested for LAW AZ-101 Per TFCOUP Rev. 2 - Alkali Variation (cont'd).

\begin{tabular}{|c|c|c|c|c|c|c|c|c|c|c|c|c|c|c|c|c|}
\hline $\begin{array}{l}\text { Glass } \\
\text { Oxides }\end{array}$ & \begin{tabular}{|c|} 
Pretreated \\
LAW \\
AZ-101 + \\
recycled \\
$\mathrm{SO}_{3}$
\end{tabular} & $\begin{array}{c}\text { Delta to } \\
\text { LAW } \\
\text { B83 }\end{array}$ & \multicolumn{2}{|c|}{$\begin{array}{l}\text { Glass Former } \\
\text { Additives for } \\
\text { LAWB93 }\end{array}$} & \multirow{2}{*}{\begin{tabular}{|c|}
$\begin{array}{c}\text { LAWB93 } \\
\text { (target) }\end{array}$ \\
$7.17 \%$ \\
\end{tabular}} & \multirow{2}{*}{\begin{tabular}{|c|}
$\begin{array}{c}\text { LAWB93S4 } \\
\text { (target) }\end{array}$ \\
$7.17 \%$ \\
\end{tabular}} & \multirow{2}{*}{\begin{tabular}{|c|} 
Delta to \\
LAW \\
B83 \\
\\
- \\
\end{tabular}} & \multicolumn{2}{|c|}{$\begin{array}{c}\text { Glass Former } \\
\text { Additives for } \\
\text { LAWB94 }\end{array}$} & \multirow{2}{*}{\begin{tabular}{|c|}
$\begin{array}{c}\text { LAWB94 } \\
\text { (target) }\end{array}$ \\
$7.17 \%$ \\
\end{tabular}} & \multirow{2}{*}{\begin{tabular}{|c}
$\begin{array}{c}\text { LAWB94S4 } \\
\text { (target) }\end{array}$ \\
$7.17 \%$ \\
\end{tabular}} & \multirow{2}{*}{$\begin{array}{c}\begin{array}{c}\text { Delta to } \\
\text { LAW } \\
\text { B83 }\end{array} \\
-\end{array}$} & \multicolumn{2}{|c|}{$\begin{array}{c}\text { Glass Former } \\
\text { Additives for } \\
\text { LAWB95 }\end{array}$} & \multirow{2}{*}{$\begin{array}{c}\begin{array}{c}\text { LAWB95 } \\
\text { (target) }\end{array} \\
7.17 \%\end{array}$} & \multirow{2}{*}{\begin{tabular}{|c}
$\begin{array}{c}\text { LAWB95S4 } \\
\text { (target) }\end{array}$ \\
$7.17 \%$ \\
\end{tabular}} \\
\hline Loading & $7.17 \%$ & - & $100 \%$ & $92.83 \%$ & & & & $100 \%$ & $92.83 \%$ & & & & $100 \%$ & $92.83 \%$ & & \\
\hline $\mathrm{Al}_{2} \mathrm{O}_{3}$ & 0.71 & - & 5.90 & 5.48 & 6.18 & 6.18 & - & 5.90 & 5.48 & 6.18 & 6.18 & - & 5.90 & 5.48 & 6.18 & 6.18 \\
\hline $\mathrm{B}_{2} \mathrm{O}_{3}$ & 0.00 & - & 10.80 & 10.03 & 10.03 & 10.03 & - & 10.80 & 10.03 & 10.03 & 10.03 & - & 10.80 & 10.03 & 10.03 & 10.03 \\
\hline $\mathrm{CaO}$ & 0.00 & - & 7.30 & 6.78 & 6.78 & 6.78 & - & 7.30 & 6.78 & 6.78 & 6.78 & - & 7.30 & 6.78 & 6.78 & 6.78 \\
\hline $\mathrm{Cr}_{2} \mathrm{O}_{3}$ & 0.04 & - & - & - & 0.04 & 0.04 & - & - & - & 0.04 & 0.04 & - & - & - & 0.04 & 0.04 \\
\hline $\mathrm{Fe}_{2} \mathrm{O}_{3}$ & 0.00 & - & 5.70 & 5.29 & 5.29 & 5.29 & - & 5.70 & 5.29 & 5.29 & 5.29 & - & 5.70 & 5.29 & 5.29 & 5.29 \\
\hline $\mathrm{K}_{2} \mathrm{O}$ & 0.19 & - & - & - & 0.19 & 0.19 & - & - & - & 0.19 & 0.19 & - & - & - & 0.19 & 0.19 \\
\hline $\mathrm{La}_{2} \mathrm{O}_{3}$ & 0.00 & - & - & - & 0.00 & 0.00 & - & - & - & 0.00 & 0.00 & - & - & - & 0.00 & 0.00 \\
\hline $\mathrm{Li}_{2} \mathrm{O}$ & - & 0.35 & 5.02 & 4.66 & 4.66 & 4.66 & 1.05 & 5.77 & 5.36 & 5.36 & 5.36 & 1.45 & 6.20 & 5.76 & 5.76 & 5.76 \\
\hline MgO & - & - & 3.20 & 2.97 & 2.97 & 2.97 & - & 3.20 & 2.97 & 2.97 & 2.97 & - & 3.20 & 2.97 & 2.97 & 2.97 \\
\hline $\mathrm{Na}_{2} \mathrm{O}$ & 5.47 & -0.70 & -0.75 & -0.70 & 4.78 & 4.78 & -2.09 & -2.25 & -2.09 & 3.38 & 3.38 & -3.02 & -3.25 & -3.02 & 2.45 & 2.45 \\
\hline $\mathrm{SiO}_{2}$ & - & 0.34 & 52.72 & 48.94 & 48.94 & 48.94 & 1.04 & 53.47 & 49.64 & 49.64 & 49.64 & 1.57 & 54.04 & 50.17 & 50.17 & 50.17 \\
\hline $\mathrm{TiO}_{2}$ & - & - & 1.50 & 1.39 & 1.39 & 1.39 & - & 1.50 & 1.39 & 1.39 & 1.39 & - & 1.50 & 1.39 & 1.39 & 1.39 \\
\hline $\mathrm{ZnO}$ & - & - & 5.21 & 4.84 & 4.84 & 4.84 & - & 5.21 & 4.84 & 4.84 & 4.84 & - & 5.21 & 4.84 & 4.84 & 4.84 \\
\hline $\mathrm{ZrO}_{2}$ & - & - & 3.40 & 3.16 & 3.16 & 3.16 & - & 3.40 & 3.16 & 3.16 & 3.16 & - & 3.40 & 3.16 & 3.16 & 3.16 \\
\hline $\mathrm{Cl}$ & 0.01 & - & - & - & 0.01 & 0.01 & - & - & - & 0.01 & 0.01 & - & - & - & 0.01 & 0.01 \\
\hline $\mathrm{F}$ & 0.06 & - & - & - & 0.06 & 0.06 & - & - & - & 0.06 & 0.06 & - & - & - & 0.06 & 0.06 \\
\hline $\mathrm{P}_{2} \mathrm{O}_{5}$ & 0.04 & - & - & - & 0.04 & 0.04 & - & - & - & 0.04 & 0.04 & - & - & - & 0.04 & 0.04 \\
\hline $\mathrm{SO}_{3}$ & 0.65 & - & - & - & 0.65 & 4.50 & - & - & - & 0.65 & 4.50 & - & - & - & 0.65 & 4.50 \\
\hline $\mathrm{Re}_{2} \mathrm{O}_{7}$ & - & - & - & - & 0.10 & 0.10 & - & - & - & 0.10 & 0.10 & - & - & - & 0.10 & 0.10 \\
\hline SUM & 7.17 & 0.00 & 100.00 & 92.83 & 100.10 & 103.95 & 0.00 & 100.00 & 92.83 & 100.10 & 103.95 & 0.00 & 100.00 & 92.83 & 100.10 & 103.95 \\
\hline
\end{tabular}


Table 3.13. Composition of LAW AZ-102 (Batch 2b) Per TFCOUP Rev. 2 and Waste Contribution to Glass.

\begin{tabular}{|l|c|c|c|c|c|}
\hline $\begin{array}{l}\text { Glass } \\
\text { Oxides }\end{array}$ & $\begin{array}{c}\text { LAW } \\
\text { AZ-102 } \\
\text { Batch 2b }\end{array}$ & $\begin{array}{c}\text { LAW AZ-102 } \\
\text { Waste } \\
\text { Contribution } \\
\text { to Glass (wt\%) }\end{array}$ & $\begin{array}{c}\text { Pretreated } \\
\text { LAW AZ-102 }\end{array}$ & $\begin{array}{c}\text { Recycled } \\
\text { off gas } \mathrm{SO}_{3}\end{array}$ & $\begin{array}{c}\text { Pretreated LAW } \\
\text { AZ-102 } \\
\text { +recycled } \mathrm{SO}_{3}\end{array}$ \\
\hline Loading & $100 \%$ & $6.65 \%$ & $6.92 \%$ & - & $7.37 \%$ \\
\hline $\mathrm{Al}_{2} \mathrm{O}_{3}$ & 2.90 & 0.19 & 0.19 & - & 0.19 \\
\hline $\mathrm{CaO}$ & 0.01 & 0.00 & 0.00 & - & 0.00 \\
\hline $\mathrm{Cr}_{2} \mathrm{O}_{3}$ & 1.49 & 0.10 & 0.10 & - & 0.10 \\
\hline $\mathrm{Fe}_{2} \mathrm{O}_{3}$ & 0.02 & 0.00 & 0.00 & - & 0.00 \\
\hline $\mathrm{K}_{2} \mathrm{O}$ & 3.83 & 0.26 & 0.26 & - & 0.26 \\
\hline $\mathrm{Na}_{2} \mathrm{O}$ & 75.17 & 5.00 & 5.27 & 0.20 & 5.46 \\
\hline $\mathrm{NiO}^{\mathrm{ClO}}$ & 0.00 & 0.00 & 0.00 & - & 0.00 \\
\hline $\mathrm{Cl}$ & 0.01 & 0.00 & 0.00 & - & 0.00 \\
\hline $\mathrm{F}$ & 1.07 & 0.07 & 0.07 & - & 0.07 \\
\hline $\mathrm{P}_{2} \mathrm{O}_{5}$ & 0.10 & 0.01 & 0.01 & - & 0.01 \\
\hline $\mathrm{SO} \mathrm{S}_{3}$ & 15.39 & 1.02 & 1.02 & 0.26 & 1.28 \\
\hline $\mathrm{SUM}$ & 100.00 & 6.65 & 6.92 & 0.45 & 7.37 \\
\hline
\end{tabular}


Table 3.14. Composition of Glasses Tested for LAW AZ-102 Per TFCOUP Rev. 2.

\begin{tabular}{|c|c|c|c|c|c|c|c|c|c|c|c|c|c|c|c|c|}
\hline $\begin{array}{l}\text { Glass } \\
\text { Oxides }\end{array}$ & \begin{tabular}{|c|} 
Pretreated \\
LAW \\
AZ-102
\end{tabular} & $\begin{array}{r}\text { Glass } \\
\text { Addit } \\
\text { LAI }\end{array}$ & $\begin{array}{l}\text { Former } \\
\text { ves for } \\
\text { JB61 }\end{array}$ & $\begin{array}{c}\text { LAWB61 } \\
\text { (target) }\end{array}$ & $\begin{array}{c}\text { LAWB61 } \\
\text { S4 } \\
\text { (target) }\end{array}$ & $\begin{array}{c}\text { Delta to } \\
\text { LAWB61 }\end{array}$ & $\begin{array}{r}\text { Glass } \\
\text { Addit } \\
\text { LA }\end{array}$ & $\begin{array}{l}\text { Former } \\
\text { ves for } \\
\text { VB62 }\end{array}$ & $\begin{array}{c}\text { LAWB62 } \\
\text { (target) }\end{array}$ & $\begin{array}{c}\text { LAWB62 } \\
\text { S4 } \\
\text { (target) }\end{array}$ & $\begin{array}{c}\text { Delta to } \\
\text { LAWB61 }\end{array}$ & $\begin{array}{r}\text { Glass } \\
\text { Addit } \\
\text { LAI }\end{array}$ & $\begin{array}{l}\text { Former } \\
\text { ves for } \\
\text { JB63 }\end{array}$ & $\begin{array}{c}\text { LAWB63 } \\
\text { (target) }\end{array}$ & $\begin{array}{c}\text { LAWB63 } \\
\text { S0 } \\
\text { (target) }\end{array}$ & $\begin{array}{c}\text { LAWB63 } \\
\text { S4 } \\
\text { (target) }\end{array}$ \\
\hline Loading & $7.37 \%$ & - & $92.63 \%$ & $7.37 \%$ & $7.37 \%$ & - & - & $92.63 \%$ & $7.37 \%$ & $7.37 \%$ & - & - & $92.63 \%$ & $7.37 \%$ & $7.37 \%$ & $7.37 \%$ \\
\hline $\mathrm{Al}_{2} \mathrm{O}_{3}$ & 0.19 & 6.45 & 5.97 & 6.17 & 6.17 & - & 6.45 & 5.97 & 6.17 & 6.17 & 0.38 & 6.86 & 6.35 & 6.55 & 6.55 & 6.55 \\
\hline $\mathrm{B}_{2} \mathrm{O}_{3}$ & 0.00 & 10.70 & 9.91 & 9.91 & 9.91 & - & 10.70 & 9.91 & 9.91 & 9.91 & - & 10.70 & 9.91 & 9.91 & 9.91 & 9.91 \\
\hline $\mathrm{CaO}$ & 0.00 & 7.20 & 6.67 & 6.67 & 6.67 & 5.28 & 12.90 & 11.95 & 11.95 & 11.95 & 2.64 & 10.05 & 9.31 & 9.31 & 9.31 & 9.31 \\
\hline $\mathrm{Cr}_{2} \mathrm{O}_{3}$ & 0.10 & - & - & 0.10 & 0.10 & - & - & - & 0.10 & 0.10 & - & - & - & 0.10 & 0.10 & 0.10 \\
\hline $\mathrm{Fe}_{2} \mathrm{O}_{3}$ & 0.00 & 5.70 & 5.28 & 5.28 & 5.28 & -5.28 & 0.00 & 0.00 & 0.00 & 0.00 & -5.28 & 0.00 & 0.00 & 0.00 & 0.00 & 0.00 \\
\hline $\mathrm{K}_{2} \mathrm{O}$ & 0.26 & - & - & 0.26 & 0.26 & - & - & - & 0.26 & 0.26 & - & - & - & 0.26 & 0.26 & 0.26 \\
\hline $\mathrm{Li}_{2} \mathrm{O}$ & - & 6.25 & 5.79 & 5.79 & 5.79 & 0.00 & 6.25 & 5.79 & 5.79 & 5.79 & -0.76 & 5.43 & 5.03 & 5.03 & 5.03 & 5.03 \\
\hline $\mathrm{MgO}$ & - & 3.20 & 2.96 & 2.96 & 2.96 & - & 3.20 & 2.96 & 2.96 & 2.96 & - & 3.20 & 2.96 & 2.96 & 2.96 & 2.96 \\
\hline $\mathrm{Na}_{2} \mathrm{O}$ & 5.46 & - & - & 5.46 & 5.46 & - & - & - & 5.46 & 5.46 & - & - & - & 5.46 & 5.46 & 5.46 \\
\hline $\mathrm{SiO}_{2}$ & - & 52.20 & 48.35 & 48.35 & 48.35 & - & 52.20 & 48.35 & 48.35 & 48.35 & 0.38 & 52.61 & 48.73 & 48.73 & 48.73 & 48.73 \\
\hline $\mathrm{TiO}_{2}$ & - & 1.50 & 1.39 & 1.39 & 1.39 & - & 1.50 & 1.39 & 1.39 & 1.39 & - & 1.50 & 1.39 & 1.39 & 1.39 & 1.39 \\
\hline $\mathrm{ZnO}$ & - & 3.40 & 3.15 & 3.15 & 3.15 & - & 3.40 & 3.15 & 3.15 & 3.15 & 2.64 & 6.25 & 5.79 & 5.79 & 5.79 & 5.79 \\
\hline $\mathrm{ZrO}_{2}$ & - & 3.40 & 3.15 & 3.15 & 3.15 & - & 3.40 & 3.15 & 3.15 & 3.15 & - & 3.40 & 3.15 & 3.15 & 3.15 & 3.15 \\
\hline $\mathrm{Cl}$ & 0.00 & - & - & 0.00 & 0.00 & - & - & - & 0.00 & 0.00 & - & - & - & 0.00 & 0.00 & 0.00 \\
\hline $\mathrm{F}$ & 0.07 & - & - & 0.07 & 0.07 & - & - & - & 0.07 & 0.07 & - & - & - & 0.07 & 0.07 & 0.07 \\
\hline $\mathrm{P}_{2} \mathrm{O}_{5}$ & 0.01 & - & - & 0.01 & 0.01 & - & - & - & 0.01 & 0.01 & - & - & - & 0.01 & 0.01 & 0.01 \\
\hline $\mathrm{SO}_{3}$ & 1.28 & - & - & 1.28 & 4.50 & - & - & - & 1.28 & 4.50 & - & - & - & 1.28 & 0.00 & 4.50 \\
\hline $\mathrm{Re}_{2} \mathrm{O}_{7}$ & - & - & - & 0.10 & 0.10 & - & - & - & 0.10 & 0.10 & - & - & - & 0.10 & 0.10 & 0.10 \\
\hline SUM & 7.37 & 100.00 & 92.63 & 100.10 & 103.32 & 0.00 & 100.00 & 92.63 & 100.10 & 103.32 & 0.00 & 100.00 & 92.63 & 100.10 & 98.82 & 103.32 \\
\hline
\end{tabular}


Table 3.14. Composition of Glasses Tested for LAW AZ-102 Per TFCOUP Rev. 2 (cont'd).

\begin{tabular}{|c|c|c|c|c|c|c|c|c|c|c|c|c|c|c|c|c|c|}
\hline \begin{tabular}{|l} 
Glass \\
Oxides
\end{tabular} & \begin{tabular}{|c|} 
Pretreated \\
LAW \\
AZ-102
\end{tabular} & $\begin{array}{c}\text { Delta to } \\
\text { LAWB61 }\end{array}$ & $\begin{array}{r}\text { Glass F } \\
\text { Additiv } \\
\text { LAW }\end{array}$ & $\begin{array}{l}\text { ormer } \\
\text { res for } \\
\text { B64 }\end{array}$ & $\begin{array}{c}\text { LAWB64 } \\
\text { (target) }\end{array}$ & $\begin{array}{c}\text { LAWB64S4 } \\
\text { (target) }\end{array}$ & $\begin{array}{c}\text { LAWB64S0 } \\
\text { (target) }\end{array}$ & $\begin{array}{c}\text { Delta to } \\
\text { LAWB61 }\end{array}$ & $\begin{array}{r}\text { Glass F } \\
\text { Additi } \\
\text { LAW }\end{array}$ & $\begin{array}{l}\text { ormer } \\
\text { ves for } \\
\text { B65 }\end{array}$ & $\begin{array}{c}\text { LAWB65 } \\
\text { (target) }\end{array}$ & $\begin{array}{c}\text { LAWB65S4 } \\
\text { (target) }\end{array}$ & $\begin{array}{c}\text { Delta to } \\
\text { LAWB61 }\end{array}$ & $\begin{array}{r}\text { Glass F } \\
\text { Additiv } \\
\text { LAW }\end{array}$ & $\begin{array}{l}\text { ormer } \\
\text { ves for } \\
\text { B66 }\end{array}$ & $\begin{array}{c}\text { LAWB66 } \\
\text { (target) }\end{array}$ & $\begin{array}{c}\text { LAWB66S4 } \\
\text { (target) }\end{array}$ \\
\hline Loading & $7.37 \%$ & - & $100.00 \%$ & $92.63 \%$ & $7.37 \%$ & $7.37 \%$ & $7.37 \%$ & - & $100.00 \%$ & $92.63 \%$ & $7.37 \%$ & $7.37 \%$ & - & $100.00 \%$ & $92.63 \%$ & $7.37 \%$ & $7.37 \%$ \\
\hline $\mathrm{Al}_{2} \mathrm{O}_{3}$ & 0.19 & - & 6.45 & 5.97 & 6.17 & 6.17 & 6.17 & - & 6.45 & 5.97 & 6.17 & 6.17 & - & 6.45 & 5.97 & 6.17 & 6.17 \\
\hline $\mathrm{B}_{2} \mathrm{O}_{3}$ & 0.00 & - & 10.70 & 9.91 & 9.91 & 9.91 & 9.91 & - & 10.70 & 9.91 & 9.91 & 9.91 & - & 10.70 & 9.91 & 9.91 & 9.91 \\
\hline $\mathrm{CaO}$ & 0.00 & - & 7.20 & 6.67 & 6.67 & 6.67 & 6.67 & - & 7.20 & 6.67 & 6.67 & 6.67 & 1.50 & 8.82 & 8.17 & 8.17 & 8.17 \\
\hline $\mathrm{Cr}_{2} \mathrm{O}_{3}$ & 0.10 & - & - & - & 0.10 & 0.10 & 0.10 & - & - & - & 0.10 & 0.10 & - & - & - & 0.10 & 0.10 \\
\hline $\mathrm{Fe}_{2} \mathrm{O}_{3}$ & 0.00 & -2.00 & 3.54 & 3.28 & 3.28 & 3.28 & 3.28 & - & 5.70 & 5.28 & 5.28 & 5.28 & - & 5.70 & 5.28 & 5.28 & 5.28 \\
\hline $\mathrm{K}_{2} \mathrm{O}$ & 0.26 & - & - & - & 0.26 & 0.26 & 0.26 & - & - & - & 0.26 & 0.26 & - & - & - & 0.26 & 0.26 \\
\hline $\mathrm{Li}_{2} \mathrm{O}$ & - & - & 6.25 & 5.79 & 5.79 & 5.79 & 5.79 & -1.50 & 4.63 & 4.29 & 4.29 & 4.29 & -1.50 & 4.63 & 4.29 & 4.29 & 4.29 \\
\hline $\mathrm{MgO}$ & - & - & 3.20 & 2.96 & 2.96 & 2.96 & 2.96 & - & 3.20 & 2.96 & 2.96 & 2.96 & - & 3.20 & 2.96 & 2.96 & 2.96 \\
\hline $\mathrm{Na}_{2} \mathrm{O}$ & 5.46 & - & - & - & 5.46 & 5.46 & 5.46 & - & - & - & 5.46 & 5.46 & - & - & - & 5.46 & 5.46 \\
\hline $\mathrm{SiO}_{2}$ & - & - & 52.20 & 48.35 & 48.35 & 48.35 & 48.35 & - & 52.20 & 48.35 & 48.35 & 48.35 & - & 52.20 & 48.35 & 48.35 & 48.35 \\
\hline $\mathrm{TiO}_{2}$ & - & - & 1.50 & 1.39 & 1.39 & 1.39 & 1.39 & - & 1.50 & 1.39 & 1.39 & 1.39 & - & 1.50 & 1.39 & 1.39 & 1.39 \\
\hline $\mathrm{ZnO}$ & - & 2.00 & 5.56 & 5.15 & 5.15 & 5.15 & 5.15 & 1.50 & 5.02 & 4.65 & 4.65 & 4.65 & - & 3.40 & 3.15 & 3.15 & 3.15 \\
\hline $\mathrm{ZrO}_{2}$ & - & - & 3.40 & 3.15 & 3.15 & 3.15 & 3.15 & - & 3.40 & 3.15 & 3.15 & 3.15 & - & 3.40 & 3.15 & 3.15 & 3.15 \\
\hline $\mathrm{Cl}$ & 0.00 & - & - & - & 0.00 & 0.00 & 0.00 & - & - & - & 0.00 & 0.00 & - & - & - & 0.00 & 0.00 \\
\hline$F$ & 0.07 & - & - & - & 0.07 & 0.07 & 0.07 & - & - & - & 0.07 & 0.07 & - & - & - & 0.07 & 0.07 \\
\hline $\mathrm{P}_{2} \mathrm{O}_{5}$ & 0.01 & - & - & - & 0.01 & 0.01 & 0.01 & - & - & - & 0.01 & 0.01 & - & - & - & 0.01 & 0.01 \\
\hline $\mathrm{SO}_{3}$ & 1.28 & - & - & - & 1.28 & 4.50 & 0.00 & - & - & - & 1.28 & 4.50 & - & - & - & 1.28 & 4.50 \\
\hline $\mathrm{Re}_{2} \mathrm{O}_{7}$ & - & - & - & - & 0.10 & 0.10 & 0.10 & - & - & - & 0.10 & 0.10 & - & - & - & 0.10 & 0.10 \\
\hline SUM & 7.37 & 0.00 & 100.00 & 92.63 & 100.10 & 103.32 & 98.82 & 0.00 & 100.00 & 92.63 & 100.10 & 103.32 & 0.00 & 100.00 & 92.63 & 100.10 & 103.32 \\
\hline
\end{tabular}


Table 3.14. Composition of Glasses Tested for LAW AZ-102 Per TFCOUP Rev. 2 (cont'd).

\begin{tabular}{|c|c|c|c|c|c|c|c|c|c|c|c|}
\hline $\begin{array}{l}\text { Glass } \\
\text { Oxides }\end{array}$ & $\begin{array}{c}\text { Pretreated } \\
\text { LAW } \\
\text { AZ-102 }\end{array}$ & \begin{tabular}{|c|} 
Delta to \\
LAWB61
\end{tabular} & $\begin{array}{r}\text { Glass } \\
\text { Addit } \\
\text { LAI }\end{array}$ & $\begin{array}{l}\text { ormer } \\
\text { es for } \\
\text { B67 }\end{array}$ & $\begin{array}{c}\text { LAWB67 } \\
\text { (target) }\end{array}$ & $\begin{array}{l}\text { LAWB67S4 } \\
\text { (target) }\end{array}$ & $\begin{array}{c}\text { Delta to } \\
\text { LAWB61 }\end{array}$ & $\begin{array}{r}\text { Glass For } \\
\text { for } \mathrm{L}\end{array}$ & $\begin{array}{l}\text { Additives } \\
\text { JB68 }\end{array}$ & $\begin{array}{c}\text { LAWB68 } \\
\text { (target) }\end{array}$ & $\begin{array}{c}\text { LAWB68S4 } \\
\text { (target) }\end{array}$ \\
\hline Loading & $7.37 \%$ & - & $100.00 \%$ & $92.63 \%$ & $7.37 \%$ & $7.37 \%$ & - & $100.00 \%$ & $92.63 \%$ & $7.37 \%$ & $7.37 \%$ \\
\hline $\mathrm{Al}_{2} \mathrm{O} 3$ & 0.19 & - & 6.45 & 5.97 & 6.17 & 6.17 & - & 6.45 & 5.97 & 6.17 & 6.17 \\
\hline $\mathrm{B}_{2} \mathrm{O}_{3}$ & 0.00 & - & 10.70 & 9.91 & 9.91 & 9.91 & -1.50 & 9.08 & 8.41 & 8.41 & 8.41 \\
\hline $\mathrm{CaO}$ & 0.00 & -1.50 & 5.58 & 5.17 & 5.17 & 5.17 & 1.50 & 8.82 & 8.17 & 8.17 & 8.17 \\
\hline $\mathrm{Cr}_{2} \mathrm{O}_{3}$ & 0.10 & - & - & - & 0.10 & 0.10 & - & - & - & 0.10 & 0.10 \\
\hline $\mathrm{Fe}_{2} \mathrm{O}_{3}$ & 0.00 & - & 5.70 & 5.28 & 5.28 & 5.28 & - & 5.70 & 5.28 & 5.28 & 5.28 \\
\hline $\mathrm{K}_{2} \mathrm{O}$ & 0.26 & - & - & - & 0.26 & 0.26 & - & - & - & 0.26 & 0.26 \\
\hline $\mathrm{Li}_{2} \mathrm{O}$ & - & -1.50 & 4.63 & 4.29 & 4.29 & 4.29 & -1.50 & 4.63 & 4.29 & 4.29 & 4.29 \\
\hline $\mathrm{MgO}$ & - & - & 3.20 & 2.96 & 2.96 & 2.96 & - & 3.20 & 2.96 & 2.96 & 2.96 \\
\hline $\mathrm{Na}_{2} \mathrm{O}$ & 5.46 & - & - & - & 5.46 & 5.46 & - & - & - & 5.46 & 5.46 \\
\hline $\mathrm{SiO}_{2}$ & - & - & 52.20 & 48.35 & 48.35 & 48.35 & - & 52.20 & 48.35 & 48.35 & 48.35 \\
\hline $\mathrm{TiO}_{2}$ & - & - & 1.50 & 1.39 & 1.39 & 1.39 & - & 1.50 & 1.39 & 1.39 & 1.39 \\
\hline $\mathrm{ZnO}$ & - & - & 3.40 & 3.15 & 3.15 & 3.15 & 1.50 & 5.02 & 4.65 & 4.65 & 4.65 \\
\hline $\mathrm{ZrO}_{2}$ & - & - & 3.40 & 3.15 & 3.15 & 3.15 & - & 3.40 & 3.15 & 3.15 & 3.15 \\
\hline $\mathrm{Cl}$ & 0.00 & - & - & - & 0.00 & 0.00 & - & - & - & 0.00 & 0.00 \\
\hline$F$ & 0.07 & - & - & - & 0.07 & 0.07 & - & - & - & 0.07 & 0.07 \\
\hline $\mathrm{P}_{2} \mathrm{O}_{5}$ & 0.01 & 3.00 & 3.24 & 3.00 & 3.01 & 3.01 & - & - & - & 0.01 & 0.01 \\
\hline $\mathrm{SO}_{3}$ & 1.28 & - & - & - & 1.28 & 4.50 & - & - & - & 1.28 & 4.50 \\
\hline $\mathrm{Re}_{2} \mathrm{O}_{7}$ & - & - & - & - & 0.10 & 0.10 & - & - & - & 0.10 & 0.10 \\
\hline SUM & 7.37 & 0.00 & 100.00 & 92.63 & 100.10 & 103.32 & 0.00 & 100.00 & 92.63 & 100.10 & 103.32 \\
\hline
\end{tabular}


Table 3.14. Composition of Glasses Tested for LAW AZ-102 Per TFCOUP Rev. 2 (cont'd).

\begin{tabular}{|c|c|c|c|c|c|c|c|c|c|c|c|c|c|}
\hline $\begin{array}{l}\text { Glass } \\
\text { Oxides }\end{array}$ & $\begin{array}{c}\text { Pretreated } \\
\text { LAW AZ-102 }\end{array}$ & $\begin{array}{c}\text { Delta to } \\
\text { LAW } \\
\text { B61 }\end{array}$ & $\begin{array}{r}\text { Glass } \\
\text { Additi } \\
\text { LAW }\end{array}$ & $\begin{array}{l}\text { ormer } \\
\text { ves for } \\
\text { B73 }\end{array}$ & $\begin{array}{l}\text { LAWB73 } \\
\text { (target) }\end{array}$ & $\begin{array}{c}\text { LAWB73S4 } \\
\text { (target) }\end{array}$ & $\begin{array}{c}\text { LAWB73S0 } \\
\text { (target) }\end{array}$ & $\begin{array}{c}\text { Delta to } \\
\text { LAW } \\
\text { B61 }\end{array}$ & $\begin{array}{r}\text { Glass F } \\
\text { Additi } \\
\text { LAW } \\
\end{array}$ & $\begin{array}{l}\text { ormer } \\
\text { ves for } \\
\text { B74 }\end{array}$ & $\begin{array}{c}\text { LAWB74 } \\
\text { (target) }\end{array}$ & $\begin{array}{c}\text { LAWB74 } \\
\text { S4 } \\
\text { (target) }\end{array}$ & $\begin{array}{c}\text { LAWB74 } \\
\text { S0 } \\
\text { (target) }\end{array}$ \\
\hline Loading & $7.37 \%$ & - & $100.00 \%$ & $92.63 \%$ & $7.37 \%$ & $7.37 \%$ & $7.37 \%$ & - & $100.00 \%$ & $92.63 \%$ & $7.37 \%$ & $7.37 \%$ & $7.37 \%$ \\
\hline $\mathrm{Al}_{2} \mathrm{O}_{3}$ & 0.19 & 0.00 & 6.45 & 5.97 & 6.17 & 6.17 & 6.17 & 0.00 & 6.45 & 5.97 & 6.17 & 6.17 & 6.17 \\
\hline $\mathrm{B}_{2} \mathrm{O}_{3}$ & 0.00 & 0.00 & 10.70 & 9.91 & 9.91 & 9.91 & 9.91 & 0.39 & 11.12 & 10.30 & 10.30 & 10.30 & 10.30 \\
\hline $\mathrm{CaO}$ & 0.00 & 2.64 & 10.05 & 9.31 & 9.31 & 9.31 & 9.31 & 1.99 & 9.35 & 8.66 & 8.66 & 8.66 & 8.66 \\
\hline $\mathrm{Cr}_{2} \mathrm{O}_{3}$ & 0.10 & - & - & - & 0.10 & 0.10 & 0.10 & - & - & - & 0.10 & 0.10 & 0.10 \\
\hline $\mathrm{Fe}_{2} \mathrm{O}_{3}$ & 0.00 & -3.38 & 2.05 & 1.90 & 1.90 & 1.90 & 1.90 & -3.38 & 2.05 & 1.90 & 1.90 & 1.90 & 1.90 \\
\hline $\mathrm{K}_{2} \mathrm{O}$ & 0.26 & - & - & - & 0.26 & 0.26 & 0.26 & - & - & - & 0.26 & 0.26 & 0.26 \\
\hline $\mathrm{Li}_{2} \mathrm{O}$ & - & -0.76 & 5.43 & 5.03 & 5.03 & 5.03 & 5.03 & -0.50 & 5.71 & 5.29 & 5.29 & 5.29 & 5.29 \\
\hline $\mathrm{MgO}$ & - & - & 3.20 & 2.96 & 2.96 & 2.96 & 2.96 & - & 3.20 & 2.96 & 2.96 & 2.96 & 2.96 \\
\hline $\mathrm{Na}_{2} \mathrm{O}$ & 5.46 & - & - & - & 5.46 & 5.46 & 5.46 & - & - & - & 5.46 & 5.46 & 5.46 \\
\hline $\mathrm{SiO}_{2}$ & - & 0.00 & 52.20 & 48.35 & 48.35 & 48.35 & 48.35 & 0.00 & 52.20 & 48.35 & 48.35 & 48.35 & 48.35 \\
\hline $\mathrm{TiO}_{2}$ & - & - & 1.50 & 1.39 & 1.39 & 1.39 & 1.39 & - & 1.50 & 1.39 & 1.39 & 1.39 & 1.39 \\
\hline $\mathrm{ZnO}$ & - & 1.50 & 5.02 & 4.65 & 4.65 & 4.65 & 4.65 & 1.50 & 5.02 & 4.65 & 4.65 & 4.65 & 4.65 \\
\hline $\mathrm{ZrO}_{2}$ & - & - & 3.40 & 3.15 & 3.15 & 3.15 & 3.15 & - & 3.40 & 3.15 & 3.15 & 3.15 & 3.15 \\
\hline $\mathrm{Cl}$ & 0.00 & - & - & - & 0.00 & 0.00 & 0.00 & - & - & - & 0.00 & 0.00 & 0.00 \\
\hline $\mathrm{F}$ & 0.07 & - & - & - & 0.07 & 0.07 & 0.07 & - & - & - & 0.07 & 0.07 & 0.07 \\
\hline $\mathrm{P}_{2} \mathrm{O}_{5}$ & 0.01 & - & - & - & 0.01 & 0.01 & 0.01 & - & - & - & 0.01 & 0.01 & 0.01 \\
\hline $\mathrm{SO}_{3}$ & 1.28 & - & - & - & 1.28 & 4.50 & 0.00 & - & - & - & 1.28 & 4.50 & 0.00 \\
\hline $\mathrm{Re}_{2} \mathrm{O}_{7}$ & - & - & - & - & 0.10 & 0.10 & 0.10 & - & - & - & 0.10 & 0.10 & 0.10 \\
\hline SUM & 7.37 & 0.00 & 100.00 & 92.63 & 100.10 & 103.32 & 98.82 & 0.00 & 100.00 & 92.63 & 100.10 & 103.32 & 98.82 \\
\hline
\end{tabular}


Table 3.14. Composition of Glasses Tested for LAW AZ-102 Per TFCOUP Rev. 2 (cont'd).

\begin{tabular}{|c|c|c|c|c|c|c|c|c|c|c|c|c|c|}
\hline $\begin{array}{l}\text { Glass } \\
\text { Oxides }\end{array}$ & \begin{tabular}{|c|} 
Pretreated \\
LAW \\
AZ-102
\end{tabular} & $\begin{array}{c}\text { Delta to } \\
\text { LAWB61 }\end{array}$ & $\begin{array}{r}\text { Glass } \\
\text { Additi } \\
\text { LAV }\end{array}$ & $\begin{array}{l}\text { ormer } \\
\text { ves for } \\
\text { B75 }\end{array}$ & $\begin{array}{l}\text { LAWB75 } \\
\text { (target) }\end{array}$ & $\begin{array}{c}\text { LAWB75S4 } \\
\text { (target) }\end{array}$ & $\begin{array}{c}\text { LAWB75S0 } \\
\text { (target) }\end{array}$ & $\begin{array}{c}\text { Delta to } \\
\text { LAWB61 }\end{array}$ & $\begin{array}{r}\text { Glass F } \\
\text { Additiv } \\
\text { LAW }\end{array}$ & $\begin{array}{l}\text { ormer } \\
\text { ves for } \\
\text { JB76 }\end{array}$ & $\begin{array}{c}\text { LAWB76 } \\
\text { (target) }\end{array}$ & $\begin{array}{c}\text { LAWB76S4 } \\
\text { (target) }\end{array}$ & $\begin{array}{c}\text { LAWB76S0 } \\
\text { (target) }\end{array}$ \\
\hline Loading & $7.37 \%$ & - & $100.00 \%$ & $92.63 \%$ & $7.37 \%$ & $7.37 \%$ & $7.37 \%$ & - & $100.00 \%$ & $92.63 \%$ & $7.37 \%$ & $7.37 \%$ & $7.37 \%$ \\
\hline $\mathrm{Al}_{2} \mathrm{O}_{3}$ & 0.19 & - & 6.45 & 5.97 & 6.17 & 6.17 & 6.17 & - & 6.45 & 5.97 & 6.17 & 6.17 & 6.17 \\
\hline $\mathrm{B}_{2} \mathrm{O}_{3}$ & 0.00 & 1.85 & 12.70 & 11.76 & 11.76 & 11.76 & 11.76 & 1.85 & 12.70 & 11.76 & 11.76 & 11.76 & 11.76 \\
\hline $\mathrm{CaO}$ & 0.00 & 1.99 & 9.35 & 8.66 & 8.66 & 8.66 & 8.66 & 1.99 & 9.35 & 8.66 & 8.66 & 8.66 & 8.66 \\
\hline $\mathrm{Cr}_{2} \mathrm{O}_{3}$ & 0.10 & - & - & - & 0.10 & 0.10 & 0.10 & - & - & - & 0.10 & 0.10 & 0.10 \\
\hline $\mathrm{Fe}_{2} \mathrm{O}_{3}$ & 0.00 & -3.38 & 2.05 & 1.90 & 1.90 & 1.90 & 1.90 & -3.38 & 2.05 & 1.90 & 1.90 & 1.90 & 1.90 \\
\hline $\mathrm{K}_{2} \mathrm{O}$ & 0.26 & - & - & - & 0.26 & 0.26 & 0.26 & - & - & - & 0.26 & 0.26 & 0.26 \\
\hline $\mathrm{Li}_{2} \mathrm{O}$ & - & -0.50 & 5.71 & 5.29 & 5.29 & 5.29 & 5.29 & - & 6.25 & 5.79 & 5.79 & 5.79 & 5.79 \\
\hline $\mathrm{MgO}$ & - & -1.46 & 1.62 & 1.50 & 1.50 & 1.50 & 1.50 & -1.46 & 1.62 & 1.50 & 1.50 & 1.50 & 1.50 \\
\hline $\mathrm{Na}_{2} \mathrm{O}$ & 5.46 & - & - & - & 5.46 & 5.46 & 5.46 & - & - & - & 5.46 & 5.46 & 5.46 \\
\hline $\mathrm{SiO}_{2}$ & - & - & 52.20 & 48.35 & 48.35 & 48.35 & 48.35 & 0.89 & 53.16 & 49.24 & 49.24 & 49.24 & 49.24 \\
\hline $\mathrm{TiO}_{2}$ & - & - & 1.50 & 1.39 & 1.39 & 1.39 & 1.39 & -1.39 & 0.00 & 0.00 & 0.00 & 0.00 & 0.00 \\
\hline $\mathrm{ZnO}$ & - & 1.50 & 5.02 & 4.65 & 4.65 & 4.65 & 4.65 & 1.50 & 5.02 & 4.65 & 4.65 & 4.65 & 4.65 \\
\hline $\mathrm{ZrO}_{2}$ & - & - & 3.40 & 3.15 & 3.15 & 3.15 & 3.15 & - & 3.40 & 3.15 & 3.15 & 3.15 & 3.15 \\
\hline $\mathrm{Cl}$ & 0.00 & - & - & - & 0.00 & 0.00 & 0.00 & - & - & - & 0.00 & 0.00 & 0.00 \\
\hline $\mathrm{F}$ & 0.07 & - & - & - & 0.07 & 0.07 & 0.07 & - & - & - & 0.07 & 0.07 & 0.07 \\
\hline $\mathrm{P}_{2} \mathrm{O}_{5}$ & 0.01 & - & - & - & 0.01 & 0.01 & 0.01 & - & - & - & 0.01 & 0.01 & 0.01 \\
\hline $\mathrm{SO}_{3}$ & 1.28 & - & - & - & 1.28 & 4.50 & 0.00 & - & - & - & 1.28 & 4.50 & 0.00 \\
\hline $\mathrm{Re}_{2} \mathrm{O}_{7}$ & & - & - & - & 0.10 & 0.10 & 0.10 & - & - & - & 0.10 & 0.10 & 0.10 \\
\hline SUM & 7.37 & 0.00 & 100.00 & 92.63 & 100.10 & 103.32 & 98.82 & 0.00 & 100.00 & 92.63 & 100.10 & 103.32 & 98.82 \\
\hline
\end{tabular}


Table 3.15. Composition of LAW AN-107 Per TFCOUP Rev. 2 and Composition of Glass LAWC22.

\begin{tabular}{|c|c|c|c|c|c|c|}
\hline \multirow{2}{*}{$\begin{array}{l}\text { Glass } \\
\text { Oxides } \\
\text { Loading }\end{array}$} & \multirow{2}{*}{$\begin{array}{c}\text { Composition } \\
\text { of LAW } \\
\text { AN-107 } \\
\text { Batch } 7 \\
100.00 \% \\
\end{array}$} & \multirow{2}{*}{$\begin{array}{c}\text { LAW AN- } \\
107 \text { Waste } \\
\text { Contribution } \\
\text { to Glass } \\
13.83 \%\end{array}$} & \multirow{2}{*}{$\begin{array}{c}\text { Pretreated } \\
\text { LAW } \\
\text { AN-107 } \\
16.09 \%\end{array}$} & \multicolumn{2}{|c|}{$\begin{array}{c}\text { Glass Former Additives } \\
\text { for LAWC22 }\end{array}$} & \multirow{2}{*}{$\begin{array}{c}\text { C22AN107 } \\
\text { (target) }\end{array}$} \\
\hline & & & & $100.00 \%$ & $83.91 \%$ & \\
\hline $\mathrm{Al}_{2} \mathrm{O}_{3}$ & 4.08 & 0.56 & 0.56 & 6.60 & 5.54 & 6.10 \\
\hline $\mathrm{B}_{2} \mathrm{O}_{3}$ & - & - & 0.00 & 12.00 & 10.07 & 10.07 \\
\hline $\mathrm{CaO}$ & 0.25 & 0.03 & 0.03 & 6.05 & 5.08 & 5.11 \\
\hline $\mathrm{Cr}_{2} \mathrm{O}_{3}$ & 0.14 & 0.02 & 0.02 & - & - & 0.02 \\
\hline $\mathrm{Fe}_{2} \mathrm{O}_{3}$ & 1.25 & 0.17 & 0.17 & 6.45 & 5.41 & 5.58 \\
\hline $\mathrm{K}_{2} \mathrm{O}$ & 0.62 & 0.09 & 0.09 & - & - & 0.09 \\
\hline $\mathrm{Li}_{2} \mathrm{O}$ & - & - & 0.00 & 2.99 & 2.51 & 2.51 \\
\hline $\mathrm{MgO}$ & - & - & 0.00 & 1.80 & 1.51 & 1.51 \\
\hline $\mathrm{Na}_{2} \mathrm{O}$ & 88.65 & 12.26 & 14.42 & - & 0.00 & 14.42 \\
\hline $\mathrm{NiO}$ & 0.20 & 0.03 & 0.03 & - & - & 0.03 \\
\hline $\mathrm{PbO}$ & 0.13 & 0.02 & 0.02 & - & - & 0.02 \\
\hline $\mathrm{SiO}_{2}$ & - & - & 0.00 & 55.50 & 46.57 & 46.57 \\
\hline $\mathrm{TiO}_{2}$ & - & - & 0.00 & 1.36 & 1.14 & 1.14 \\
\hline $\mathrm{ZnO}$ & - & - & 0.00 & 3.65 & 3.06 & 3.06 \\
\hline $\mathrm{ZrO}_{2}$ & - & - & 0.00 & 3.60 & 3.02 & 3.02 \\
\hline $\mathrm{Cl}$ & 0.58 & 0.08 & 0.08 & - & - & 0.08 \\
\hline $\mathrm{F}$ & 1.05 & 0.14 & 0.14 & - & - & 0.14 \\
\hline $\mathrm{P}_{2} \mathrm{O}_{5}$ & 0.85 & 0.12 & 0.12 & - & - & 0.12 \\
\hline $\mathrm{SO}_{3}$ & 2.21 & 0.31 & 0.31 & - & - & 0.31 \\
\hline $\mathrm{Re}_{2} \mathrm{O}_{7}$ & - & - & 0.10 & - & - & 0.10 \\
\hline SUM & 100.00 & 13.83 & 16.09 & 100.00 & 83.91 & 100.00 \\
\hline
\end{tabular}


Table 3.16. Composition of LAW AN-102 Per TFCOUP Rev. 2 and Waste Contribution to Glass.

\begin{tabular}{|c|c|c|c|c|c|}
\hline $\begin{array}{l}\text { Glass } \\
\text { Oxides }\end{array}$ & $\begin{array}{c}\text { Composition of } \\
\text { LAW AN-102 } \\
\text { Batch } 3\end{array}$ & $\begin{array}{l}\text { LAW AN-102 } \\
\text { Waste } \\
\text { Contribution } \\
\text { to Glass }\end{array}$ & $\begin{array}{c}\text { Pretreated } \\
\text { LAW AN-102 }\end{array}$ & $\begin{array}{c}\text { Recycled } \\
\text { Off Gas } \\
\mathrm{SO}_{3}\end{array}$ & $\begin{array}{l}\text { Pretreated LAW } \\
\quad \text { AN-102 } \\
+ \text { recycled } \mathrm{SO}_{3}\end{array}$ \\
\hline Loading & - & $11.82 \%$ & $13.59 \%$ & - & $13.76 \%$ \\
\hline $\mathrm{Al}_{2} \mathrm{O}_{3}$ & 7.26 & 0.86 & 0.86 & - & 0.86 \\
\hline $\mathrm{BaO}$ & 0.01 & 0.00 & 0.00 & - & 0.00 \\
\hline $\mathrm{CaO}$ & 0.22 & 0.03 & 0.03 & - & 0.03 \\
\hline $\mathrm{CdO}$ & 0.02 & 0.00 & 0.00 & - & 0.00 \\
\hline $\mathrm{Cr}_{2} \mathrm{O}_{3}$ & 0.15 & 0.02 & 0.02 & - & 0.02 \\
\hline $\mathrm{Fe}_{2} \mathrm{O}_{3}$ & 0.08 & 0.01 & 0.01 & - & 0.01 \\
\hline $\mathrm{K}_{2} \mathrm{O}$ & 1.15 & 0.14 & 0.14 & - & 0.14 \\
\hline $\mathrm{Na}_{2} \mathrm{O}$ & 85.41 & 10.10 & 11.88 & 0.07 & 11.96 \\
\hline $\mathrm{NiO}$ & 0.13 & 0.02 & 0.00 & - & 0.00 \\
\hline $\mathrm{PbO}$ & 0.05 & 0.01 & 0.00 & - & 0.00 \\
\hline $\mathrm{Cl}$ & 0.94 & 0.11 & 0.11 & - & 0.11 \\
\hline$F$ & 0.46 & 0.05 & 0.05 & - & 0.05 \\
\hline $\mathrm{P}_{2} \mathrm{O}_{5}$ & 0.90 & 0.11 & 0.11 & - & 0.11 \\
\hline $\mathrm{SO}_{3}$ & 3.23 & 0.38 & 0.38 & 0.10 & 0.48 \\
\hline SUM & 100.00 & 11.82 & 13.59 & 0.17 & 13.76 \\
\hline
\end{tabular}


Table 3.17. Composition of LAWC21 for AN-102 Composition Per TFCOUP Rev. 2.

\begin{tabular}{|c|c|c|c|c|c|c|}
\hline $\begin{array}{l}\text { Glass } \\
\text { Oxides }\end{array}$ & $\begin{array}{c}\text { Composition } \\
\text { of Pretreated } \\
\text { LAW } \\
\text { AN-102 }\end{array}$ & \begin{tabular}{|c|} 
Pretreated \\
LAW AN- \\
$102+$ \\
recycled $\mathrm{SO}_{3}$
\end{tabular} & $\begin{array}{c}\text { Glass Former } \\
\text { Additives }\end{array}$ & $\begin{array}{c}\text { C21REV2 } \\
\text { (target) }\end{array}$ & $\begin{array}{c}\text { C21REV2S0 } \\
\text { (target) }\end{array}$ & $\begin{array}{c}\text { C21REV2S2 } \\
\text { (target) }\end{array}$ \\
\hline Loading & $13.59 \%$ & $13.76 \%$ & $86.24 \%$ & $13.76 \%$ & $13.76 \%$ & $13.76 \%$ \\
\hline $\mathrm{Al}_{2} \mathrm{O}_{3}$ & 0.03 & 0.86 & 5.26 & 6.12 & 6.12 & 6.12 \\
\hline $\mathrm{B}_{2} \mathrm{O}_{3}$ & 0.00 & 0.00 & 10.05 & 10.05 & 10.05 & 10.05 \\
\hline $\mathrm{CaO}$ & 0.02 & 0.03 & 6.38 & 6.41 & 6.41 & 6.41 \\
\hline $\mathrm{Cr}_{2} \mathrm{O}_{3}$ & 0.01 & 0.02 & - & 0.02 & 0.02 & 0.02 \\
\hline $\mathrm{Fe}_{2} \mathrm{O}_{3}$ & - & 0.01 & 6.42 & 6.43 & 6.43 & 6.43 \\
\hline $\mathrm{K}_{2} \mathrm{O}$ & 0.14 & 0.14 & - & 0.14 & 0.14 & 0.14 \\
\hline $\mathrm{Li}_{2} \mathrm{O}$ & - & - & 2.73 & 2.73 & 2.73 & 2.73 \\
\hline $\mathrm{MgO}$ & - & - & 1.50 & 1.50 & 1.50 & 1.50 \\
\hline $\mathrm{Na}_{2} \mathrm{O}$ & 11.88 & 11.96 & - & 11.96 & 11.96 & 11.96 \\
\hline $\mathrm{SiO}_{2}$ & - & - & 46.74 & 46.74 & 46.74 & 46.74 \\
\hline $\mathrm{TiO}_{2}$ & - & - & 1.12 & 1.12 & 1.12 & 1.12 \\
\hline $\mathrm{ZnO}$ & - & - & 3.02 & 3.02 & 3.02 & 3.02 \\
\hline $\mathrm{ZrO}_{2}$ & - & - & 3.02 & 3.02 & 3.02 & 3.02 \\
\hline $\mathrm{Cl}$ & 0.11 & 0.11 & - & 0.11 & 0.11 & 0.11 \\
\hline $\mathrm{F}$ & 0.05 & 0.05 & - & 0.05 & 0.05 & 0.05 \\
\hline $\mathrm{P}_{2} \mathrm{O}_{5}$ & 0.11 & 0.11 & 0.00 & 0.11 & 0.11 & 0.11 \\
\hline $\mathrm{SO}_{3}$ & 0.38 & 0.48 & - & 0.48 & 0.00 & 2.50 \\
\hline $\mathrm{Re}_{2} \mathrm{O}_{7}$ & - & - & - & 0.10 & 0.10 & 0.10 \\
\hline SUM & 13.59 & 13.76 & 86.24 & 100.10 & 99.62 & 102.12 \\
\hline
\end{tabular}


Table 3.18. Composition of Glasses Tested for LAW AN-102 Per TFCOUP Rev. 2.

\begin{tabular}{|c|c|c|c|c|c|c|c|c|c|c|}
\hline $\begin{array}{c}\text { Glass } \\
\text { Oxides }\end{array}$ & 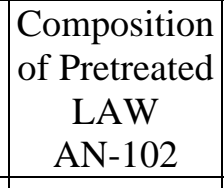 & $\begin{array}{c}\text { Pretreated } \\
\text { LAW } \\
\text { AN-102 + } \\
\text { recycled } \mathrm{SO}_{3}\end{array}$ & \begin{tabular}{|c|} 
Glass Former \\
Additives \\
for \\
LAWC26 \\
\end{tabular} & $\begin{array}{c}\text { Delta } \\
\text { to } \\
\text { LAWC21 }\end{array}$ & $\begin{array}{c}\text { LAWC26 } \\
\text { (target) }\end{array}$ & $\begin{array}{c}\text { LAWC26S2 } \\
\text { (target) }\end{array}$ & \begin{tabular}{||c|} 
Glass Former \\
Additives \\
For \\
LAWC27 \\
\end{tabular} & $\begin{array}{l}\text { Delta } \\
\text { to } \\
\text { C21 }\end{array}$ & $\begin{array}{c}\text { LAWC27 } \\
\text { (target) }\end{array}$ & $\begin{array}{c}\text { LAWC27S2 } \\
\text { (target) }\end{array}$ \\
\hline Loading & $13.59 \%$ & $13.76 \%$ & $86.24 \%$ & - & $13.76 \%$ & $13.59 \%$ & $86.24 \%$ & - & $13.76 \%$ & $13.59 \%$ \\
\hline $\mathrm{Al}_{2} \mathrm{O}_{3}$ & 0.86 & 0.86 & 5.26 & - & 6.12 & 6.13 & 5.26 & - & 6.12 & 6.13 \\
\hline $\mathrm{B}_{2} \mathrm{O}_{3}$ & 0.00 & 0.00 & 13.26 & 3.21 & 13.26 & 13.33 & 12.19 & 2.14 & 12.19 & 12.25 \\
\hline $\mathrm{CaO}$ & 0.03 & 0.03 & 6.38 & - & 6.41 & 6.41 & 8.52 & 2.14 & 8.55 & 8.57 \\
\hline $\mathrm{Cr}_{2} \mathrm{O}_{3}$ & 0.02 & 0.02 & - & - & 0.02 & 0.02 & - & - & 0.02 & 0.02 \\
\hline $\mathrm{Fe}_{2} \mathrm{O}_{3}$ & 0.01 & 0.01 & 0.00 & -6.42 & 0.01 & 0.01 & 0.00 & -6.42 & 0.01 & 0.01 \\
\hline $\mathrm{K}_{2} \mathrm{O}$ & 0.14 & 0.14 & - & - & 0.14 & 0.15 & - & - & 0.14 & 0.15 \\
\hline $\mathrm{Li}_{2} \mathrm{O}$ & - & - & 2.73 & - & 2.73 & 2.74 & 2.73 & - & 2.73 & 2.74 \\
\hline MgO & - & - & 1.50 & - & 1.50 & 1.51 & 1.50 & - & 1.51 & 1.51 \\
\hline $\mathrm{Na}_{2} \mathrm{O}$ & 11.88 & 11.96 & - & - & 11.96 & 11.88 & - & - & 11.96 & 11.88 \\
\hline $\mathrm{SiO}_{2}$ & - & - & 49.95 & 3.21 & 49.95 & 50.00 & 48.88 & 2.14 & 48.88 & 48.92 \\
\hline $\mathrm{TiO}_{2}$ & - & - & 1.12 & - & 1.12 & 1.12 & 1.12 & - & 1.12 & 1.12 \\
\hline $\mathrm{ZnO}$ & - & - & 3.02 & - & 3.02 & 3.02 & 3.02 & - & 3.02 & 3.02 \\
\hline $\mathrm{ZrO}_{2}$ & - & - & 3.02 & - & 3.02 & 3.02 & 3.02 & - & 3.02 & 3.02 \\
\hline $\mathrm{Cl}$ & 0.11 & 0.11 & - & - & 0.11 & 0.12 & - & - & 0.11 & 0.12 \\
\hline $\mathrm{F}$ & 0.05 & 0.05 & - & - & 0.05 & 0.06 & - & - & 0.05 & 0.06 \\
\hline $\mathrm{P}_{2} \mathrm{O}_{5}$ & 0.11 & 0.11 & 0.00 & - & 0.11 & 0.12 & 0.00 & - & 0.11 & 0.12 \\
\hline $\mathrm{SO}_{3}$ & 0.38 & 0.48 & - & - & 0.48 & 2.50 & - & - & 0.48 & 2.50 \\
\hline $\mathrm{Re}_{2} \mathrm{O}_{7}$ & - & - & - & - & 0.10 & 0.10 & - & - & 0.10 & 0.10 \\
\hline SUM & 13.59 & 13.76 & 86.24 & 0.00 & 100.10 & 102.25 & 86.24 & 0.00 & 100.10 & 102.25 \\
\hline
\end{tabular}


Table 3.18. Composition of Glasses Tested for LAW AN-102 Per TFCOUP Rev. 2 (cont'd).

\begin{tabular}{|c|c|c|c|c|c|c|c|c|c|c|c|}
\hline $\begin{array}{l}\text { Glass } \\
\text { Oxides }\end{array}$ & $\begin{array}{c}\text { Composition } \\
\text { of Pretreated } \\
\text { LAW } \\
\text { AN-102 }\end{array}$ & $\begin{array}{c}\text { Pretreated } \\
\text { LAW } \\
\text { AN-102 + } \\
\text { recycled } \\
\mathrm{SO}_{3} \\
\end{array}$ & $\begin{array}{c}\text { Glass } \\
\text { Former } \\
\text { Additives } \\
\text { for } \\
\text { LAWC28 } \\
\end{array}$ & $\begin{array}{c}\text { Delta } \\
\text { to } \\
\text { LAWC21 }\end{array}$ & $\begin{array}{l}\text { LAWC28 } \\
\text { (target) }\end{array}$ & $\begin{array}{c}\text { LAWC28S2 } \\
\text { (target) }\end{array}$ & $\begin{array}{c}\text { Glass } \\
\text { Former } \\
\text { Additives } \\
\text { for } \\
\text { LAWC29 } \\
\end{array}$ & $\begin{array}{c}\text { Delta } \\
\text { to } \\
\text { LAWC21 }\end{array}$ & $\begin{array}{c}\text { LAWC29 } \\
\text { (target) }\end{array}$ & $\begin{array}{c}\text { LAWC29S2 } \\
\text { (target) }\end{array}$ & $\begin{array}{c}\text { LAWC29S0 } \\
\text { (target) }\end{array}$ \\
\hline Loading & $13.59 \%$ & $13.76 \%$ & $86.24 \%$ & - & $13.76 \%$ & $13.59 \%$ & $86.24 \%$ & - & $13.76 \%$ & $13.76 \%$ & $13.76 \%$ \\
\hline $\mathrm{Al}_{2} \mathrm{O}_{3}$ & 0.86 & 0.86 & 5.26 & - & 6.12 & 6.13 & 5.69 & 0.43 & 6.55 & 6.55 & 6.55 \\
\hline $\mathrm{B}_{2} \mathrm{O}_{3}$ & 0.00 & 0.00 & 10.05 & - & 10.05 & 10.10 & 10.05 & - & 10.05 & 10.05 & 10.05 \\
\hline $\mathrm{CaO}$ & 0.03 & 0.03 & 12.80 & 6.42 & 12.82 & 12.89 & 9.59 & 3.21 & 9.62 & 9.62 & 9.62 \\
\hline $\mathrm{Cr}_{2} \mathrm{O}_{3}$ & 0.02 & 0.02 & - & - & 0.02 & 0.02 & - & - & 0.02 & 0.02 & 0.02 \\
\hline $\mathrm{Fe}_{2} \mathrm{O}_{3}$ & 0.01 & 0.01 & 0.00 & -6.42 & 0.01 & 0.01 & 0.00 & -6.42 & 0.01 & 0.01 & 0.01 \\
\hline $\mathrm{K}_{2} \mathrm{O}$ & 0.14 & 0.14 & - & - & 0.14 & 0.15 & - & - & 0.14 & 0.14 & 0.14 \\
\hline $\mathrm{Li}_{2} \mathrm{O}$ & - & - & 2.73 & - & 2.74 & 2.74 & 2.73 & - & 2.74 & 2.74 & 2.74 \\
\hline $\mathrm{MgO}$ & - & - & 1.50 & - & 1.51 & 1.51 & 1.50 & - & 1.51 & 1.51 & 1.51 \\
\hline $\mathrm{Na}_{2} \mathrm{O}$ & 11.88 & 11.96 & - & - & 11.96 & 11.88 & - & - & 11.96 & 11.96 & 11.96 \\
\hline $\mathrm{SiO}_{2}$ & - & - & 46.74 & - & 46.74 & 46.76 & 47.18 & 0.43 & 47.18 & 47.18 & 47.18 \\
\hline $\mathrm{TiO}_{2}$ & - & - & 1.12 & - & 1.12 & 1.12 & 1.12 & - & 1.12 & 1.12 & 1.12 \\
\hline $\mathrm{ZnO}$ & - & - & 3.02 & - & 3.02 & 3.02 & 5.36 & 2.35 & 5.36 & 5.36 & 5.36 \\
\hline $\mathrm{ZrO}_{2}$ & - & - & 3.02 & - & 3.02 & 3.02 & 3.02 & - & 3.02 & 3.02 & 3.02 \\
\hline $\mathrm{Cl}$ & 0.11 & 0.11 & - & - & 0.11 & 0.12 & - & - & 0.11 & 0.11 & 0.11 \\
\hline $\mathrm{F}$ & 0.05 & 0.05 & 0.00 & - & 0.05 & 0.06 & - & - & 0.05 & 0.05 & 0.05 \\
\hline $\mathrm{P}_{2} \mathrm{O}_{5}$ & 0.11 & 0.11 & - & - & 0.11 & 0.12 & 0.00 & - & 0.11 & 0.11 & 0.11 \\
\hline $\mathrm{SO}_{3}$ & 0.38 & 0.478 & - & - & 0.48 & 2.5 & - & - & 0.48 & 2.50 & 0.00 \\
\hline $\mathrm{Re}_{2} \mathrm{O}_{7}$ & - & - & 86.24 & - & 0.10 & 0.10 & - & - & 0.10 & 0.10 & 0.10 \\
\hline SUM & - & 13.76 & - & 0.00 & 100.1 & 102.25 & 86.24 & 0.00 & 100.10 & 102.12 & 99.62 \\
\hline
\end{tabular}


Table 3.18. Composition of Glasses Tested for LAW AN-102 Per TFCOUP Rev. 2 (cont'd).

\begin{tabular}{|c|c|c|c|c|c|c|c|c|c|c|c|}
\hline \begin{tabular}{||l} 
Glass \\
Oxides
\end{tabular} & \begin{tabular}{|c|} 
Pretreated \\
LAW \\
AN-102 + \\
recycled \\
$\mathrm{SO}_{3}$ \\
\end{tabular} & \begin{tabular}{||c|} 
Glass \\
Former \\
Additives for \\
LAWC30
\end{tabular} & $\begin{array}{c}\text { Delta } \\
\text { to } \\
\text { LAW } \\
\text { C21 }\end{array}$ & $\begin{array}{c}\text { LAWC30 } \\
\text { (target) }\end{array}$ & $\begin{array}{c}\text { LAWC30S2 } \\
\text { (target) }\end{array}$ & \begin{tabular}{||c} 
Glass \\
Former \\
Additives for \\
LAWC31
\end{tabular} & $\begin{array}{c}\begin{array}{c}\text { Delta } \\
\text { to }\end{array} \\
\text { LAW } \\
\text { C21 }\end{array}$ & $\begin{array}{c}\text { LAWC31 } \\
\text { (target) }\end{array}$ & $\begin{array}{c}\text { LAWC31R1 } \\
\text { (target) }\end{array}$ & $\begin{array}{c}\text { LAWC31S0 } \\
\text { (target) }\end{array}$ & $\begin{array}{c}\text { LAWC31S2 } \\
\text { (target) }\end{array}$ \\
\hline Loading & $13.76 \%$ & $86.24 \%$ & - & $13.76 \%$ & $13.76 \%$ & $86.24 \%$ & - & $13.76 \%$ & $13.76 \%$ & $13.76 \%$ & $13.76 \%$ \\
\hline $\mathrm{Al}_{2} \mathrm{O}_{3}$ & 0.86 & 5.26 & - & 6.12 & 6.12 & 5.26 & - & 6.12 & 6.12 & 6.12 & 6.12 \\
\hline $\mathrm{B}_{2} \mathrm{O}_{3}$ & 0.00 & 10.05 & - & 10.05 & 10.05 & 10.05 & - & 10.05 & 10.05 & 10.05 & 10.05 \\
\hline $\mathrm{CaO}$ & 0.03 & 6.38 & - & 6.41 & 6.41 & 7.38 & 1.00 & 7.41 & 7.41 & 7.41 & 7.41 \\
\hline $\mathrm{Cr}_{2} \mathrm{O}_{3}$ & 0.02 & - & - & 0.02 & 0.02 & - & - & 0.02 & 0.02 & 0.02 & 0.02 \\
\hline $\mathrm{Fe}_{2} \mathrm{O}_{3}$ & 0.01 & 4.09 & -2.33 & 4.10 & 4.10 & 4.42 & -2.00 & 4.42 & 4.42 & 4.42 & 4.42 \\
\hline $\mathrm{K}_{2} \mathrm{O}$ & 0.14 & - & - & 0.14 & 0.14 & - & - & 0.14 & 0.14 & 0.14 & 0.14 \\
\hline $\mathrm{Li}_{2} \mathrm{O}$ & - & 2.73 & - & 2.73 & 2.73 & 2.73 & - & 2.73 & 2.73 & 2.73 & 2.73 \\
\hline $\mathrm{MgO}$ & - & 1.50 & - & 1.50 & 1.50 & 1.50 & - & 1.50 & 1.50 & 1.50 & 1.50 \\
\hline $\mathrm{Na}_{2} \mathrm{O}$ & 11.96 & - & - & 11.96 & 11.96 & - & - & 11.96 & 11.96 & 11.96 & 11.96 \\
\hline $\mathrm{SiO}_{2}$ & - & 46.74 & - & 46.74 & 46.74 & 46.74 & - & 46.74 & 46.74 & 46.74 & 46.74 \\
\hline $\mathrm{SnO}_{2}$ & - & - & - & - & & - & - & - & - & - & - \\
\hline $\mathrm{TiO}_{2}$ & - & 1.12 & - & 1.12 & 1.12 & 1.12 & - & 1.12 & 1.12 & 1.12 & 1.12 \\
\hline $\mathrm{ZnO}$ & - & 5.35 & 2.33 & 5.35 & 5.35 & 4.02 & 1.00 & 4.02 & 4.02 & 4.02 & 4.02 \\
\hline $\mathrm{ZrO}_{2}$ & - & 3.02 & - & 3.02 & 3.02 & 3.02 & - & 3.02 & 3.02 & 3.02 & 3.02 \\
\hline $\mathrm{Cl}$ & 0.11 & - & - & 0.11 & 0.11 & - & - & 0.11 & 0.11 & 0.11 & 0.11 \\
\hline $\mathrm{F}$ & 0.05 & - & - & 0.05 & 0.05 & - & - & 0.05 & 0.05 & 0.05 & 0.05 \\
\hline $\mathrm{P}_{2} \mathrm{O}_{5}$ & 0.11 & 0.00 & - & 0.11 & 0.11 & 0.00 & - & 0.11 & 0.11 & 0.11 & 0.11 \\
\hline $\mathrm{SO}_{3}$ & 0.48 & - & - & 0.48 & 2.50 & - & - & 0.48 & 0.48 & 0.00 & 2.50 \\
\hline $\mathrm{Re}_{2} \mathrm{O}_{7}$ & - & - & - & 0.10 & 0.10 & - & - & 0.10 & 0.10 & 0.10 & 0.10 \\
\hline SUM & 13.77 & 86.24 & 0.00 & 100.11 & 102.13 & 86.24 & 0.00 & 100.10 & 100.10 & 99.62 & 102.12 \\
\hline
\end{tabular}


Table 3.18. Composition of Glasses Tested for LAW AN-102 Per TFCOUP Rev. 2 (cont'd).

\begin{tabular}{|c|c|c|c|c|c|c|c|c|c|c|}
\hline $\begin{array}{l}\text { Glass } \\
\text { Oxides }\end{array}$ & $\begin{array}{c}\text { Pretreated } \\
\text { LAW } \\
\mathrm{AN}-102 \\
+ \text { recycled } \\
\mathrm{SO}_{3} \\
\end{array}$ & \begin{tabular}{||c} 
Glass Former \\
Additives for \\
LAW C32
\end{tabular} & $\begin{array}{c}\text { Delta } \\
\text { to } \\
\text { LAWC21 }\end{array}$ & $\begin{array}{c}\text { LAWC32 } \\
\text { (target) }\end{array}$ & $\begin{array}{c}\text { LAWC32S0 } \\
\text { (target) }\end{array}$ & $\begin{array}{c}\text { LAWC32S2 } \\
\text { (target) }\end{array}$ & $\begin{array}{c}\text { Glass Former } \\
\text { Additives for } \\
\text { LAWC31 }\end{array}$ & $\begin{array}{c}\text { Delta } \\
\text { to } \\
\text { LAWC21 }\end{array}$ & $\begin{array}{l}\text { LAWC33 } \\
\text { (target) }\end{array}$ & $\begin{array}{c}\text { LAWC33 } \\
\text { Renormalized }\end{array}$ \\
\hline Loading & $13.76 \%$ & $86.24 \%$ & - & $13.76 \%$ & $13.76 \%$ & $13.76 \%$ & $86.24 \%$ & - & $13.76 \%$ & - \\
\hline $\mathrm{Al}_{2} \mathrm{O}_{3}$ & 0.86 & 5.63 & 0.37 & 6.49 & 6.49 & 6.49 & 5.26 & - & 6.12 & 6.14 \\
\hline $\mathrm{B}_{2} \mathrm{O}_{3}$ & 0.00 & 10.05 & - & 10.05 & 10.05 & 10.05 & 10.05 & - & 10.05 & 10.09 \\
\hline $\mathrm{CaO}$ & 0.03 & 9.01 & 2.63 & 9.04 & 9.04 & 9.04 & 7.38 & 0.50 & 6.91 & 6.94 \\
\hline $\mathrm{Cr}_{2} \mathrm{O}_{3}$ & 0.02 & - & - & 0.02 & 0.02 & 0.02 & - & - & 0.02 & 0.02 \\
\hline $\mathrm{Fe}_{2} \mathrm{O}_{3}$ & 0.01 & 2.41 & -4.00 & 2.42 & 2.42 & 2.42 & 4.42 & -2.00 & 4.42 & 4.44 \\
\hline $\mathrm{K}_{2} \mathrm{O}$ & 0.14 & - & - & 0.14 & 0.14 & 0.14 & - & - & 0.14 & 0.14 \\
\hline $\mathrm{Li}_{2} \mathrm{O}$ & - & 2.73 & - & 2.73 & 2.73 & 2.73 & 2.73 & - & 2.73 & 2.75 \\
\hline $\mathrm{MgO}$ & - & 1.50 & - & 1.50 & 1.50 & 1.50 & 1.50 & - & 1.50 & 1.51 \\
\hline $\mathrm{Na}_{2} \mathrm{O}$ & 11.96 & - & - & 11.96 & 11.96 & 11.96 & - & - & 11.96 & 12.00 \\
\hline $\mathrm{SiO}_{2}$ & - & 46.74 & - & 46.74 & 46.74 & 46.74 & 46.74 & - & 46.74 & 46.93 \\
\hline $\mathrm{SnO}_{2}$ & - & - & - & - & - & - & - & - & - & 0.00 \\
\hline $\mathrm{TiO}_{2}$ & - & 1.12 & - & 1.12 & 1.12 & 1.12 & 1.12 & - & 1.12 & 1.13 \\
\hline $\mathrm{ZnO}$ & - & 4.02 & 1.00 & 4.02 & 4.02 & 4.02 & 4.02 & 1.00 & 4.02 & 4.04 \\
\hline $\mathrm{ZrO}_{2}$ & - & 3.02 & - & 3.02 & 3.02 & 3.02 & 3.02 & - & 3.02 & 3.03 \\
\hline $\mathrm{Cl}$ & 0.11 & - & - & 0.11 & 0.11 & 0.11 & - & - & 0.11 & 0.11 \\
\hline |F & 0.05 & - & - & 0.05 & 0.05 & 0.05 & - & - & 0.05 & 0.05 \\
\hline $\mathrm{P}_{2} \mathrm{O}_{5}$ & 0.11 & 0.00 & - & 0.11 & 0.11 & 0.11 & 0.00 & - & 0.11 & 0.11 \\
\hline $\mathrm{SO}_{3}$ & 0.48 & - & - & 0.48 & 0.00 & 2.50 & - & - & 0.48 & 0.48 \\
\hline $\mathrm{Re}_{2} \mathrm{O}_{7}$ & - & - & - & 0.10 & 0.10 & 0.10 & - & - & 0.10 & 0.10 \\
\hline SUM & 13.76 & 86.24 & 0.00 & 100.10 & 99.62 & 102.12 & 86.24 & -0.50 & 99.60 & 100.00 \\
\hline
\end{tabular}


Table 4.1. XRF Analysis of LAWA44 Glasses for LAW AN-105.

\begin{tabular}{|c|c|c|c|c|c|c|c|c|c|c|c|c|}
\hline Oxide & LAWA44S1 & - & LAWA44S1W & - & LAWA44S2 & - & LAWA44S2W & - & LAWA44SX & - & LAWA44SXW & - \\
\hline- & Target & XRF & Target & XRF & Target & XRF & Target & XRF & Target & XRF & Target & XRF \\
\hline $\mathrm{Al}_{2} \mathrm{O}_{3}$ & 6.21 & 6.14 & 6.21 & 6.13 & 6.21 & 6.20 & 6.21 & 6.16 & 6.21 & 6.15 & 6.21 & 6.17 \\
\hline $\mathrm{B}_{2} \mathrm{O}_{3}$ & 8.91 & N/A & 8.91 & N/A & 8.91 & N/A & 8.91 & N/A & 8.91 & N/A & 8.91 & N/A \\
\hline $\mathrm{CaO}$ & 2.00 & 2.09 & 2.00 & 1.99 & 2.00 & 2.02 & 2.00 & 2.00 & 2.00 & 1.97 & 2.00 & 2.01 \\
\hline $\mathrm{Cl}$ & 0.56 & 0.46 & 0.56 & 0.47 & 0.56 & 0.40 & 0.56 & 0.40 & 0.56 & 0.39 & 0.56 & 0.38 \\
\hline $\mathrm{Cr}_{2} \mathrm{O}_{3}$ & 0.02 & 0.02 & 0.02 & 0.02 & 0.02 & 0.02 & 0.02 & 0.02 & 0.02 & 0.02 & 0.02 & 0.02 \\
\hline $\mathrm{F}$ & 0.04 & N/A & 0.04 & N/A & 0.04 & N/A & 0.04 & N/A & 0.04 & N/A & 0.04 & N/A \\
\hline $\mathrm{Fe}_{2} \mathrm{O}_{3}$ & 6.98 & 7.54 & 6.98 & 7.31 & 6.98 & 7.37 & 6.98 & 7.41 & 6.98 & 6.82 & 6.98 & 7.37 \\
\hline $\mathrm{K}_{2} \mathrm{O}$ & 0.43 & 0.55 & 0.43 & 0.55 & 0.43 & 0.53 & 0.43 & 0.52 & 0.43 & 0.48 & 0.43 & 0.49 \\
\hline $\mathrm{MgO}$ & 2.00 & 1.85 & 2.00 & 1.93 & 2.00 & 1.87 & 2.00 & 1.85 & 2.00 & 2.00 & 2.00 & 1.88 \\
\hline $\mathrm{Na}_{2} \mathrm{O}$ & 20.00 & 18.13 & 20.00 & 18.39 & 20.00 & 18.69 & 20.00 & 17.77 & 21.50 & 18.85 & 21.50 & 17.27 \\
\hline $\mathrm{P}_{2} \mathrm{O}_{5}$ & 0.10 & 0.04 & 0.10 & 0.04 & 0.10 & 0.05 & 0.10 & 0.05 & 0.10 & 0.04 & 0.10 & 0.05 \\
\hline $\mathrm{Re}_{2} \mathrm{O}_{7}$ & 0.10 & 0.03 & 0.10 & 0.03 & 0.10 & 0.03 & 0.10 & 0.03 & 0.10 & 0.02 & 0.10 & 0.02 \\
\hline $\mathrm{SiO}_{2}$ & 44.58 & 45.76 & 44.58 & 46.18 & 44.58 & 46.18 & 44.58 & 46.88 & 44.58 & 46.73 & 44.58 & 47.15 \\
\hline $\mathrm{SO}_{3}$ & 1.48 & 0.54 & 1.48 & 0.51 & 2.50 & 0.54 & 2.50 & 0.53 & 2.50 & 0.62 & 2.50 & 0.58 \\
\hline $\mathrm{TiO}_{2}$ & 2.00 & 2.17 & 2.00 & 2.10 & 2.00 & 2.02 & 2.00 & 2.03 & 2.00 & 2.00 & 2.00 & 2.16 \\
\hline $\mathrm{ZnO}$ & 2.97 & 3.07 & 2.97 & 2.88 & 2.97 & 2.92 & 2.97 & 2.90 & 2.97 & 2.71 & 2.97 & 2.92 \\
\hline $\mathrm{ZrO}_{2}$ & 3.00 & 3.62 & 3.00 & 3.44 & 3.00 & 3.11 & 3.00 & 3.38 & 3.00 & 3.15 & 3.00 & 3.47 \\
\hline Sum* & 101.38 & 100.90 & 101.38 & 100.86 & 102.40 & 100.85 & 102.40 & 100.82 & 103.90 & 100.84 & 103.90 & 100.82 \\
\hline
\end{tabular}

$\mathrm{BDL}=$ Below detection limit of the instrument; N/A = Not Analyzed; Target value used for $\mathrm{B}_{2} \mathrm{O}_{3}$ and $\mathrm{Li}_{2} \mathrm{O}$; $*=\mathrm{Includes}_{2} \mathrm{O}_{3}$ and $\mathrm{Li}_{2} \mathrm{O}$ in $\mathrm{Sum}$. 
Table 4.2. XRF Analysis of Glasses for LAW AN-105 Per TFCOUP Rev. 2.

\begin{tabular}{|c|c|c|c|c|c|c|c|c|c|c|c|c|}
\hline Oxide & LAWA108S1 & - & LAWA108S2 & - & LAWA108S2W & - & LAWA108S0 & - & LAWA109S1 & - & LAWA109S1W & - \\
\hline & Target & XRF & Target & XRF & Target & XRF & Target & XRF & Target & XRF & Target & XRF \\
\hline $\mathrm{Al}_{2} \mathrm{O}_{3}$ & 6.20 & 6.08 & 6.20 & 6.42 & 6.20 & 6.50 & 6.20 & 6.02 & 6.20 & 6.01 & 6.20 & 6.16 \\
\hline $\mathrm{B}_{2} \mathrm{O}_{3}$ & 12.39 & N/A & 12.39 & N/A & 12.39 & N/A & 12.39 & N/A & 10.65 & N/A & 10.65 & N/A \\
\hline $\mathrm{CaO}$ & 1.99 & 2.11 & 1.99 & 2.01 & 1.99 & 2.02 & 1.99 & 2.16 & 1.99 & 2.04 & 1.99 & 1.99 \\
\hline $\mathrm{Cl}$ & 0.65 & 0.51 & 0.65 & 0.41 & 0.65 & 0.41 & 0.65 & 0.63 & 0.65 & 0.46 & 0.65 & 0.45 \\
\hline $\mathrm{Cr}_{2} \mathrm{O}_{3}$ & 0.02 & 0.02 & 0.02 & 0.02 & 0.02 & 0.02 & 0.02 & 0.03 & 0.02 & 0.02 & 0.02 & 0.02 \\
\hline $\mathrm{F}$ & 0.01 & N/A & 0.01 & N/A & 0.01 & N/A & 0.01 & N/A & 0.01 & N/A & 0.01 & N/A \\
\hline $\mathrm{Fe}_{2} \mathrm{O}_{3}$ & 0.00 & 0.03 & 0.00 & 0.04 & 0.00 & 0.04 & 0.00 & 0.11 & 3.49 & 3.70 & 3.49 & 3.68 \\
\hline $\mathrm{K}_{2} \mathrm{O}$ & 0.50 & 0.58 & 0.50 & 0.54 & 0.50 & 0.53 & 0.50 & 0.55 & 0.50 & 0.56 & 0.50 & 0.54 \\
\hline $\mathrm{MgO}$ & 1.99 & 1.85 & 1.99 & 1.89 & 1.99 & 1.86 & 1.99 & 1.78 & 1.99 & 1.89 & 1.99 & 1.84 \\
\hline $\mathrm{Na}_{2} \mathrm{O}$ & 20.00 & 18.26 & 20.00 & 17.95 & 20.00 & 16.72 & 20.00 & 19.05 & 20.00 & 18.61 & 20.00 & 18.15 \\
\hline $\mathrm{P}_{2} \mathrm{O}_{5}$ & 0.03 & 0.05 & 0.03 & 0.05 & 0.03 & 0.06 & 0.03 & 0.05 & 0.03 & 0.05 & 0.03 & 0.05 \\
\hline $\mathrm{Re}_{2} \mathrm{O}_{7}$ & 0.10 & 0.04 & 0.10 & 0.02 & 0.10 & 0.03 & 0.10 & 0.08 & 0.10 & 0.03 & 0.10 & 0.03 \\
\hline $\mathrm{SiO}_{2}$ & 48.04 & 49.86 & 48.04 & 50.21 & 48.04 & 51.15 & 48.04 & 49.33 & 46.30 & 47.97 & 46.30 & 48.65 \\
\hline $\mathrm{SO}_{3}$ & 1.48 & 0.66 & 2.50 & 0.70 & 2.50 & 0.65 & 0.00 & & 1.48 & 0.68 & 1.48 & 0.63 \\
\hline $\mathrm{TiO}_{2}$ & 1.99 & 2.18 & 1.99 & 2.15 & 1.99 & 2.19 & 1.99 & 2.17 & 1.99 & 2.03 & 1.99 & 2.01 \\
\hline $\mathrm{ZnO}$ & 2.96 & 3.01 & 2.96 & 2.89 & 2.96 & 2.90 & 2.96 & 3.07 & 2.96 & 2.88 & 2.96 & 2.80 \\
\hline $\mathrm{ZrO}_{2}$ & 2.99 & 3.32 & 2.99 & 3.24 & 2.99 & 3.42 & 2.99 & 3.46 & 2.99 & 3.30 & 2.99 & 3.23 \\
\hline Sum* & 101.37 & 100.95 & 102.39 & 100.93 & 102.39 & 100.89 & 99.89 & 100.87 & 101.37 & 100.90 & 101.37 & 100.86 \\
\hline
\end{tabular}


Table 4.2. XRF Analysis of Glasses for LAW AN-105 Per TFCOUP Rev. 2 (cont'd).

\begin{tabular}{|c|c|c|c|c|c|c|c|c|c|c|c|c|}
\hline Oxide & LAWA109S2 & - & LAWA109S2W & - & LAWA110S1 & - & LAWA110S1W & - & LAWA110S2 & - & LAWA110S2W & - \\
\hline & Target & XRF & Target & XRF & Target & XRF & Target & XRF & Target & XRF & Target & XRF \\
\hline $\mathrm{Al}_{2} \mathrm{O}_{3}$ & 6.20 & 6.13 & 6.20 & 6.15 & 7.36 & 7.35 & 7.36 & 7.47 & 7.36 & 7.36 & 7.36 & 7.40 \\
\hline $\mathrm{B}_{2} \mathrm{O}_{3}$ & 10.65 & N/A & 10.65 & N/A & 10.07 & N/A & 10.07 & N/A & 10.07 & N/A & 10.07 & N/A \\
\hline $\mathrm{CaO}$ & 1.99 & 2.01 & 1.99 & 2.04 & 3.16 & 3.22 & 3.16 & 3.17 & 3.16 & 3.21 & 3.16 & 3.16 \\
\hline $\mathrm{Cl}$ & 0.65 & 0.38 & 0.65 & 0.37 & 0.65 & 0.51 & 0.65 & 0.49 & 0.65 & 0.42 & 0.65 & 0.40 \\
\hline $\mathrm{Cr}_{2} \mathrm{O}_{3}$ & 0.02 & 0.02 & 0.02 & 0.02 & 0.02 & 0.02 & 0.02 & 0.02 & 0.02 & 0.02 & 0.02 & 0.02 \\
\hline $\mathrm{F}$ & 0.01 & N/A & 0.01 & N/A & 0.01 & N/A & 0.01 & N/A & 0.01 & N/A & 0.01 & N/A \\
\hline $\mathrm{Fe}_{2} \mathrm{O}_{3}$ & 3.49 & 3.71 & 3.49 & 3.83 & 3.49 & 3.79 & 3.49 & 3.78 & 3.49 & 3.74 & 3.49 & 3.74 \\
\hline $\mathrm{K}_{2} \mathrm{O}$ & 0.50 & 0.52 & 0.50 & 0.52 & 0.50 & 0.56 & 0.50 & 0.55 & 0.50 & 0.54 & 0.50 & 0.53 \\
\hline $\mathrm{MgO}$ & 1.99 & 1.93 & 1.99 & 1.93 & 1.99 & 1.92 & 1.99 & 1.85 & 1.99 & 1.85 & 1.99 & 1.87 \\
\hline $\mathrm{Na}_{2} \mathrm{O}$ & 20.00 & 18.13 & 20.00 & 16.73 & 20.00 & 18.46 & 20.00 & 17.40 & 20.00 & 17.63 & 20.00 & 17.61 \\
\hline $\mathrm{P}_{2} \mathrm{O}_{5}$ & 0.03 & 0.05 & 0.03 & 0.04 & 0.03 & 0.05 & 0.03 & 0.05 & 0.03 & 0.05 & 0.03 & 0.05 \\
\hline $\mathrm{Re}_{2} \mathrm{O}_{7}$ & 0.10 & 0.02 & 0.10 & 0.02 & 0.10 & 0.04 & 0.10 & 0.04 & 0.10 & 0.04 & 0.10 & 0.04 \\
\hline $\mathrm{SiO}_{2}$ & 46.30 & 48.44 & 46.30 & 49.40 & 44.55 & 46.13 & 44.55 & 47.16 & 44.55 & 47.08 & 44.55 & 47.21 \\
\hline $\mathrm{SO}_{3}$ & 2.50 & 0.70 & 2.50 & 0.63 & 1.48 & 0.68 & 1.48 & 0.65 & 2.50 & 0.68 & 2.50 & 0.61 \\
\hline $\mathrm{TiO}_{2}$ & 1.99 & 2.01 & 1.99 & 2.09 & 1.99 & 2.03 & 1.99 & 2.04 & 1.99 & 2.14 & 1.99 & 2.13 \\
\hline $\mathrm{ZnO}$ & 2.96 & 2.88 & 2.96 & 2.93 & 2.96 & 2.94 & 2.96 & 2.89 & 2.96 & 2.91 & 2.96 & 2.87 \\
\hline $\mathrm{ZrO}_{2}$ & 2.99 & 3.35 & 2.99 & 3.52 & 2.99 & 3.32 & 2.99 & 3.42 & 2.99 & 3.32 & 2.99 & 3.36 \\
\hline Sum* & 102.39 & 100.91 & 102.39 & 100.87 & 101.37 & 101.08 & 101.37 & 101.03 & 102.39 & 101.03 & 102.39 & 101.05 \\
\hline
\end{tabular}

$\mathrm{BDL}=$ Below detection limit of the instrument; N/A = Not Analyzed; Target value used for $\mathrm{B}_{2} \mathrm{O}_{3}$ and $\mathrm{Li}_{2} \mathrm{O}$; * $=\mathrm{Includes}_{2} \mathrm{O}_{3}$ and $\mathrm{Li} \mathrm{i}_{2} \mathrm{O}$ in Sum. 
Table 4.2. XRF Analysis of Glasses for LAW AN-105 Per TFCOUP Rev. 2 (cont'd).

\begin{tabular}{|c|c|c|c|c|c|c|c|c|c|c|c|c|}
\hline Oxide & LAWA111S1 & - & LAWA111S1W & - & LAWA111S2 & - & LAWA111S2W & - & LAWA133 & - & LAWA133S2 & - \\
\hline & Target & XRF & Target & XRF & Target & XRF & Target & $\mathrm{XRF}$ & Target & XRF & Target & XRF \\
\hline $\mathrm{Al}_{2} \mathrm{O}_{3}$ & 6.20 & 5.86 & 6.20 & 5.98 & 6.20 & 5.88 & 6.20 & 5.98 & 6.21 & 6.06 & 6.21 & 6.25 \\
\hline $\mathrm{B}_{2} \mathrm{O}_{3}$ & 8.90 & N/A & 8.90 & N/A & 8.90 & N/A & 8.90 & N/A & 8.91 & N/A & 8.91 & N/A \\
\hline $\mathrm{CaO}$ & 8.98 & 9.16 & 8.98 & 9.20 & 8.98 & 9.38 & 8.98 & 9.10 & 5.49 & 5.54 & 5.49 & 5.18 \\
\hline $\mathrm{Cl}$ & 0.65 & 0.51 & 0.65 & 0.52 & 0.65 & 0.41 & 0.65 & 0.41 & 0.56 & 0.40 & 0.56 & 0.35 \\
\hline $\mathrm{Cr}_{2} \mathrm{O}_{3}$ & 0.02 & 0.02 & 0.02 & 0.02 & 0.02 & 0.02 & 0.02 & 0.02 & 0.02 & 0.02 & 0.02 & 0.02 \\
\hline $\mathrm{F}$ & 0.01 & N/A & 0.01 & N/A & 0.01 & N/A & 0.01 & N/A & 0.04 & N/A & 0.04 & N/A \\
\hline $\mathrm{Fe}_{2} \mathrm{O}_{3}$ & 0.00 & 0.03 & 0.00 & 0.05 & 0.00 & 0.03 & 0.00 & 0.03 & 3.49 & 3.72 & 3.49 & 3.36 \\
\hline $\mathrm{K}_{2} \mathrm{O}$ & 0.50 & 0.56 & 0.50 & 0.57 & 0.50 & 0.52 & 0.50 & 0.52 & 0.43 & 0.47 & 0.43 & 0.42 \\
\hline $\mathrm{MgO}$ & 1.99 & 1.85 & 1.99 & 1.81 & 1.99 & 1.93 & 1.99 & 1.83 & 2.00 & 1.87 & 2.00 & 1.97 \\
\hline $\mathrm{Na}_{2} \mathrm{O}$ & 20.00 & 18.52 & 20.00 & 17.13 & 20.00 & 18.10 & 20.00 & 17.10 & 20.00 & 19.60 & 20.00 & 19.40 \\
\hline $\mathrm{P}_{2} \mathrm{O}_{5}$ & 0.03 & 0.05 & 0.03 & 0.06 & 0.03 & 0.06 & 0.03 & 0.06 & 0.10 & 0.13 & 0.10 & 0.14 \\
\hline $\mathrm{Re}_{2} \mathrm{O}_{7}$ & 0.10 & 0.03 & 0.10 & 0.04 & 0.10 & 0.02 & 0.10 & 0.02 & 0.10 & 0.05 & 0.10 & 0.03 \\
\hline $\mathrm{SiO}_{2}$ & 44.55 & 45.87 & 44.55 & 47.07 & 44.55 & 46.76 & 44.55 & 47.44 & 44.58 & 45.30 & 44.58 & 46.52 \\
\hline $\mathrm{SO}_{3}$ & 1.48 & 0.80 & 1.48 & 0.72 & 2.50 & 0.80 & 2.50 & 0.80 & 0.22 & 0.20 & 2.50 & 0.66 \\
\hline $\mathrm{TiO}_{2}$ & 1.99 & 2.15 & 1.99 & 2.18 & 1.99 & 2.28 & 1.99 & 2.20 & 2.00 & 2.19 & 2.00 & 2.03 \\
\hline $\mathrm{ZnO}$ & 2.96 & 3.08 & 2.96 & 3.03 & 2.96 & 3.12 & 2.96 & 2.96 & 2.97 & 2.98 & 2.97 & 2.65 \\
\hline $\mathrm{ZrO}_{2}$ & 2.99 & 3.62 & 2.99 & 3.63 & 2.99 & 3.62 & 2.99 & 3.58 & 3.00 & 3.51 & 3.00 & 3.07 \\
\hline Sum* & 101.37 & 100.99 & 101.37 & 100.89 & 102.39 & 100.91 & 102.39 & 100.92 & 100.09 & 100.95 & 102.37 & 100.97 \\
\hline
\end{tabular}

BDL = Below detection limit of the instrument; N/A = Not Analyzed; Target value used for $\mathrm{B}_{2} \mathrm{O}_{3}$ and $\mathrm{Li}_{2} \mathrm{O}$; * = Includes $\mathrm{B}_{2} \mathrm{O}_{3}$ and $\mathrm{Li}_{2} \mathrm{O}$ in $\mathrm{Sum}^{*}$. 
Table 4.2. XRF Analysis of Glasses for Tank AN-105 Per TFCOUP Rev. 2 (cont'd).

\begin{tabular}{|c|c|c|}
\hline Oxide & LAWA133S2W & - \\
\hline & Target & XRF \\
\hline $\mathrm{Al}_{2} \mathrm{O}_{3}$ & 6.21 & 6.25 \\
\hline $\mathrm{B}_{2} \mathrm{O}_{3}$ & 8.91 & N/A \\
\hline $\mathrm{CaO}$ & 5.49 & 5.32 \\
\hline $\mathrm{Cl}$ & 0.56 & 0.34 \\
\hline $\mathrm{Cr}_{2} \mathrm{O}_{3}$ & 0.02 & 0.02 \\
\hline $\mathrm{F}$ & 0.04 & N/A \\
\hline $\mathrm{Fe}_{2} \mathrm{O}_{3}$ & 3.49 & 3.60 \\
\hline $\mathrm{K}_{2} \mathrm{O}$ & 0.43 & 0.43 \\
\hline $\mathrm{MgO}$ & 2.00 & 1.87 \\
\hline $\mathrm{Na}_{2} \mathrm{O}$ & 20.00 & 18.39 \\
\hline $\mathrm{P}_{2} \mathrm{O}_{5}$ & 0.10 & 0.12 \\
\hline $\mathrm{Re}_{2} \mathrm{O}_{7}$ & 0.10 & 0.03 \\
\hline $\mathrm{SiO}_{2}$ & 44.58 & 46.77 \\
\hline $\mathrm{SO}_{3}$ & 2.50 & 0.57 \\
\hline $\mathrm{TiO}_{2}$ & 2.00 & 2.15 \\
\hline $\mathrm{ZnO}$ & 2.97 & 2.80 \\
\hline $\mathrm{ZrO}_{2}$ & 3.00 & 3.37 \\
\hline Sum* & 102.37 & 100.94 \\
\hline
\end{tabular}

$\mathrm{BDL}=$ Below detection limit of the instrument; N/A = Not Analyzed; Target value used for $\mathrm{B}_{2} \mathrm{O}_{3}$ and $\mathrm{Li}_{2} \mathrm{O}$; * $=$ Includes $\mathrm{B}_{2} \mathrm{O}_{3}$ and $\mathrm{Li}{ }_{2} \mathrm{O}$ in Sum. 
Table 4.3. XRF Analysis of LAWA88 Glass for LAW AP-101.

\begin{tabular}{|c|c|c|c|c|c|c|c|c|c|c|c|c|c|c|}
\hline Oxide & \multicolumn{2}{|c|}{ LAWA88CC } & \multicolumn{2}{|c|}{ LAWA88S1 } & \multicolumn{2}{|c|}{ LAWA88S1W } & \multicolumn{2}{|c|}{ LAWA88S2 } & \multicolumn{2}{|c|}{ LAWA88S2W } & \multicolumn{2}{|c|}{ LAWA88SX } & \multicolumn{2}{|c|}{ LAWA88SXW } \\
\hline- & Target & XRF & Target & XRF & Target & XRF & Target & XRF & Target & XRF & Target & $\mathrm{XRF}$ & Target & XRF \\
\hline $\mathrm{Al}_{2} \mathrm{O}_{3}$ & 6.08 & 5.86 & 6.08 & 6.12 & 6.08 & 6.12 & 6.08 & 6.12 & 6.08 & 6.08 & 6.08 & 5.85 & 6.08 & 6.04 \\
\hline $\mathrm{B}_{2} \mathrm{O}_{3}$ & 9.70 & N/A & 9.70 & N/A & 9.70 & N/A & 9.70 & N/A & 9.70 & N/A & 9.70 & N/A & 9.70 & N/A \\
\hline $\mathrm{CaO}$ & 1.99 & 2.03 & 1.99 & 2.02 & 1.99 & 2.02 & 1.99 & 1.97 & 1.99 & 1.98 & 1.99 & 1.99 & 1.99 & 1.99 \\
\hline $\mathrm{Cl}$ & 0.33 & 0.22 & 0.33 & 0.26 & 0.33 & 0.25 & 0.33 & 0.22 & 0.33 & 0.21 & 0.33 & 0.22 & 0.33 & 0.21 \\
\hline $\mathrm{Cr}_{2} \mathrm{O}_{3}$ & 0.01 & 0.01 & 0.01 & 0.01 & 0.01 & 0.01 & 0.01 & $\mathrm{BDL}$ & 0.01 & 0.01 & 0.01 & 0.01 & 0.01 & 0.01 \\
\hline $\mathrm{F}$ & 0.00 & N/A & 0.00 & N/A & 0.00 & N/A & 0.00 & N/A & 0.00 & N/A & 0.00 & N/A & 0.00 & N/A \\
\hline $\mathrm{Fe}_{2} \mathrm{O}_{3}$ & 5.53 & 5.91 & 5.53 & 5.77 & 5.53 & 5.91 & 5.53 & 5.60 & 5.53 & 5.80 & 5.53 & 5.72 & 5.53 & 5.89 \\
\hline $\mathrm{K}_{2} \mathrm{O}$ & 2.58 & 2.54 & 2.58 & 2.47 & 2.58 & 2.48 & 2.58 & 2.37 & 2.58 & 2.35 & 2.58 & 2.15 & 2.58 & 2.15 \\
\hline $\mathrm{MgO}$ & 1.47 & 1.45 & 1.47 & 1.38 & 1.47 & 1.32 & 1.47 & 1.41 & 1.47 & 1.39 & 1.47 & 1.34 & 1.47 & 1.27 \\
\hline $\mathrm{P}_{2} \mathrm{O}_{5}$ & 0.07 & 0.10 & 0.07 & 0.10 & 0.07 & 0.10 & 0.07 & 0.09 & 0.07 & 0.09 & 0.07 & 0.09 & 0.07 & 0.10 \\
\hline $\mathrm{Re}_{2} \mathrm{O}_{7}$ & 0.10 & 0.04 & 0.10 & 0.03 & 0.10 & 0.04 & 0.10 & 0.03 & 0.10 & 0.03 & 0.10 & 0.02 & 0.10 & 0.03 \\
\hline $\mathrm{SiO}_{2}$ & 43.99 & 44.50 & 43.99 & 45.50 & 43.99 & 45.82 & 43.99 & 46.03 & 43.99 & 46.68 & 43.99 & 45.39 & 43.99 & 46.19 \\
\hline $\mathrm{SO}_{3}$ & 0.21 & 0.20 & 1.40 & 0.71 & 1.40 & 0.69 & 2.50 & 0.75 & 2.50 & 0.65 & 2.50 & 0.85 & 2.50 & 0.75 \\
\hline $\mathrm{TiO}_{2}$ & 1.99 & 2.11 & 1.99 & 2.10 & 1.99 & 2.15 & 1.99 & 2.06 & 1.99 & 2.14 & 1.99 & 2.03 & 1.99 & 2.09 \\
\hline $\mathrm{ZnO}$ & 2.95 & 2.97 & 2.95 & 2.87 & 2.95 & 2.91 & 2.95 & 2.79 & 2.95 & 2.84 & 2.95 & 2.84 & 2.95 & 2.89 \\
\hline $\mathrm{ZrO}_{2}$ & 2.99 & 3.40 & 2.99 & 3.37 & 2.99 & 3.44 & 2.99 & 3.12 & 2.99 & 3.41 & 2.99 & 3.36 & 2.99 & 3.46 \\
\hline Sum* & 100.00 & 100.79 & 101.18 & 100.80 & 101.18 & 100.78 & 102.28 & 100.79 & 102.28 & 100.78 & 103.58 & 100.83 & 103.58 & 100.81 \\
\hline
\end{tabular}

$\mathrm{BDL}=$ Below detection limit of the instrument; N/A = Not Analyzed; Target value used for $\mathrm{B}_{2} \mathrm{O}_{3}$ and $\mathrm{Li}_{2} \mathrm{O}$; * = Includes $\mathrm{B}_{2} \mathrm{O}_{3}$ and $\mathrm{Li}_{2} \mathrm{O}$ in $\mathrm{Sum}$ 
Table 4.4. XRF Analysis of Glasses for LAW AP-101 per PNNL-13354 [8].

\begin{tabular}{|c|c|c|c|c|c|c|c|c|}
\hline \multirow{2}{*}{$\begin{array}{c}\text { Oxide } \\
- \\
\end{array}$} & \multicolumn{2}{|c|}{ LAWA130 } & \multicolumn{2}{|c|}{ LAWA134 } & \multicolumn{2}{|c|}{ LAWA135 } & \multicolumn{2}{|c|}{ LAWA136 } \\
\hline & Target & $\mathrm{XRF}$ & Target & $\mathrm{XRF}$ & Target & $\mathrm{XRF}$ & Target & $\mathrm{XRF}$ \\
\hline $\mathrm{Al}_{2} \mathrm{O}_{3}$ & 6.03 & 5.90 & 5.65 & 5.17 & 5.66 & 5.20 & 5.66 & 5.29 \\
\hline $\mathrm{B}_{2} \mathrm{O}_{3}$ & 8.95 & N/A & 9.97 & N/A & 10.10 & N/A & 10.10 & $\mathrm{~N} / \mathrm{A}$ \\
\hline $\mathrm{CaO}$ & 2.08 & 2.22 & 2.02 & 2.09 & 2.05 & 2.10 & 3.05 & 3.00 \\
\hline $\mathrm{Cl}$ & 0.20 & 0.17 & 0.20 & 0.15 & 0.19 & 0.14 & 0.19 & 0.14 \\
\hline $\mathrm{Cr}_{2} \mathrm{O}_{3}$ & 0.02 & 0.02 & 0.02 & 0.18 & 0.02 & 0.18 & 0.02 & 0.17 \\
\hline $\mathrm{Cs}_{2} \mathrm{O}$ & 0.16 & 0.19 & 0.00 & BLD & 0.00 & BLD & 0.00 & BLD \\
\hline $\mathrm{F}$ & 0.30 & N/A & 0.29 & N/A & 0.28 & N/A & 0.28 & N/A \\
\hline $\mathrm{Fe}_{2} \mathrm{O}_{3}$ & 2.86 & 3.29 & 5.63 & 6.19 & 5.70 & 6.40 & 5.70 & 6.09 \\
\hline $\mathrm{K}_{2} \mathrm{O}$ & 3.88 & 3.64 & 3.73 & 3.33 & 3.58 & 3.21 & 3.58 & 3.09 \\
\hline $\mathrm{MgO}$ & 1.18 & 0.98 & 1.50 & 1.44 & 1.52 & 1.41 & 1.52 & 1.48 \\
\hline $\mathrm{Na}_{2} \mathrm{O}$ & 18.46 & 16.65 & 17.74 & 17.77 & 17.03 & 17.20 & 17.03 & 17.24 \\
\hline $\mathrm{P}_{2} \mathrm{O}_{5}$ & 0.08 & 0.11 & 0.08 & 0.09 & 0.07 & 0.10 & 0.07 & 0.09 \\
\hline $\mathrm{Re}_{2} \mathrm{O}_{7}$ & 0.10 & 0.07 & 0.10 & 0.05 & 0.10 & 0.05 & 0.10 & 0.05 \\
\hline $\mathrm{SiO}_{2}$ & 46.09 & 47.54 & 44.78 & 44.84 & 45.34 & 45.12 & 44.34 & 45.06 \\
\hline $\mathrm{SO}_{3}$ & 0.35 & 0.33 & 0.33 & 0.28 & 0.32 & 0.27 & 0.32 & 0.27 \\
\hline $\mathrm{TiO}_{2}$ & 2.09 & 2.27 & 2.03 & 2.24 & 2.05 & 2.26 & 2.05 & 2.18 \\
\hline $\mathrm{ZnO}$ & 4.14 & 4.36 & 3.00 & 3.06 & 3.04 & 3.12 & 3.04 & 2.90 \\
\hline $\mathrm{ZrO}_{2}$ & 3.13 & 3.92 & 3.04 & 3.75 & 3.08 & 3.75 & 3.08 & 3.47 \\
\hline Sum & 100.10 & 100.59 & 100.10 & 100.60 & 100.10 & 100.61 & 100.10 & 100.62 \\
\hline
\end{tabular}

$\mathrm{BDL}=$ Below detection limit of the instrument; N/A = Not Analyzed; Target value used for $\mathrm{B}_{2} \mathrm{O}_{3}$ and $\mathrm{Li}_{2} \mathrm{O}$; * $=$ Includes $\mathrm{B}_{2} \mathrm{O}_{3}$ and $\mathrm{Li} \mathrm{i}_{2} \mathrm{O}$ in $\mathrm{Sum}$ 
Table 4.5. XRF Analysis of Glasses for LAW AW-101 Per TFCOUP Rev. 2.

\begin{tabular}{|c|c|c|c|c|c|c|c|c|}
\hline Oxide & \multicolumn{2}{|c|}{ LAWA112B14 } & \multicolumn{2}{|c|}{ LAWA112B15 } & \multicolumn{2}{|c|}{ LAWA112S1 } & \multicolumn{2}{|c|}{ LAWA112S2 } \\
\hline- & Target & XRF & Target & XRF & Target & XRF & Target & XRF \\
\hline $\mathrm{Al}_{2} \mathrm{O}_{3}$ & 6.10 & 5.96 & 6.16 & 6.04 & 6.08 & 5.72 & 6.08 & 5.83 \\
\hline $\mathrm{B}_{2} \mathrm{O}_{3}$ & 9.87 & N/A & 9.81 & N/A & 9.70 & N/A & 9.70 & N/A \\
\hline $\mathrm{CaO}$ & 7.65 & 7.74 & 7.61 & 7.67 & 7.52 & 7.73 & 7.52 & 7.94 \\
\hline $\mathrm{Cl}$ & 0.38 & 0.30 & 0.31 & 0.28 & 0.33 & 0.26 & 0.33 & 0.21 \\
\hline $\mathrm{Cr}_{2} \mathrm{O}_{3}$ & 0.02 & 0.03 & 0.01 & 0.02 & 0.01 & 0.01 & 0.01 & 0.01 \\
\hline $\mathrm{F}$ & 0.10 & N/A & 0.09 & N/A & 0.00 & N/A & 0.00 & N/A \\
\hline $\mathrm{Fe}_{2} \mathrm{O}_{3}$ & 0.00 & 0.02 & 0.00 & 0.03 & 0.00 & 0.03 & 0.00 & 0.03 \\
\hline $\mathrm{K}_{2} \mathrm{O}$ & 1.89 & 1.93 & 2.41 & 2.45 & 2.58 & 2.59 & 2.58 & 2.52 \\
\hline $\mathrm{MgO}$ & 1.48 & 1.39 & 1.48 & 1.34 & 1.48 & 1.47 & 1.48 & 1.28 \\
\hline $\mathrm{Na}_{2} \mathrm{O}$ & 20.00 & 18.76 & 20.00 & 19.22 & 20.00 & 18.59 & 20.00 & 17.73 \\
\hline $\mathrm{P}_{2} \mathrm{O}_{5}$ & 0.10 & 0.12 & 0.05 & 0.07 & 0.07 & 0.09 & 0.07 & 0.09 \\
\hline $\mathrm{Re}_{2} \mathrm{O}_{7}$ & 0.10 & 0.05 & 0.10 & 0.04 & 0.10 & 0.04 & 0.10 & 0.02 \\
\hline $\mathrm{SiO}_{2}$ & 44.26 & 45.54 & 44.02 & 44.85 & 43.99 & 44.79 & 43.99 & 45.11 \\
\hline $\mathrm{SO}_{3}$ & 0.17 & 0.16 & 0.11 & 0.11 & 1.50 & 1.10 & 2.50 & 1.15 \\
\hline $\mathrm{TiO}_{2}$ & 2.00 & 2.23 & 1.99 & 1.81 & 1.99 & 2.19 & 1.99 & 2.35 \\
\hline $\mathrm{ZnO}$ & 2.97 & 3.06 & 2.95 & 3.44 & 2.95 & 3.04 & 2.95 & 3.17 \\
\hline $\mathrm{ZrO}_{2}$ & 3.01 & 3.72 & 2.99 & 3.60 & 2.99 & 3.57 & 2.99 & 3.76 \\
\hline Sum* & 100.10 & 100.90 & 100.10 & 100.90 & 101.30 & 100.90 & 102.30 & 100.90 \\
\hline
\end{tabular}

$\mathrm{BDL}=$ Below detection limit of the instrument; N/A = Not Analyzed; Target value used for $\mathrm{B}_{2} \mathrm{O}_{3}$ and $\mathrm{Li}_{2} \mathrm{O} ; *=$ Includes $\mathrm{B}_{2} \mathrm{O}_{3}$ and $\mathrm{Li} \mathrm{O}_{2} \mathrm{O}$ in Sum 
Table 4.6. XRF Analysis of Glasses Tested for LAW AN-104.

\begin{tabular}{|c|c|c|c|c|c|c|c|c|c|c|c|c|c|c|c|c|c|c|}
\hline \multirow{2}{*}{$\begin{array}{c}\text { Oxide } \\
-\end{array}$} & \multicolumn{2}{|c|}{ LAWA102R1 } & \multicolumn{2}{|c|}{ LAWA118S1 } & \multicolumn{2}{|c|}{ LAWA118S1W } & \multicolumn{2}{|c|}{ LAWA118S2 } & \multicolumn{2}{|c|}{ LAWA118S2W } & \multicolumn{2}{|c|}{ LAWA119S1 } & \multicolumn{2}{|c|}{ LAWA119S1W } & \multicolumn{2}{|c|}{ LAWA119S2 } & \multicolumn{2}{|c|}{ LAWA119S2W } \\
\hline & Target & XRF & Target & XRF & Target & XRF & Target & XRF & Target & XRF & Target & $\mathrm{XRF}$ & Target & XRF & Target & XRF & Target & XRF \\
\hline $\mathrm{Al}_{2} \mathrm{O}_{3}$ & 6.06 & 5.57 & 6.05 & 5.83 & 6.05 & 5.82 & 6.05 & 5.52 & 6.05 & 5.53 & 6.05 & 5.88 & 6.05 & 6.00 & 6.05 & 5.97 & 6.05 & 5.94 \\
\hline $\mathrm{B}_{2} \mathrm{O}_{3}$ & 10.00 & N/A & 10.01 & N/A & 10.01 & N/A & 10.01 & N/A & 10.01 & N/A & 10.01 & N/A & 10.01 & N/A & 10.01 & N/A & 10.01 & N/A \\
\hline $\mathrm{CaO}$ & 5.06 & 5.15 & 5.07 & 5.15 & 5.07 & 5.12 & 5.07 & 5.14 & 10.48 & 5.08 & 5.07 & 10.68 & 10.48 & 10.17 & 10.48 & 10.67 & 10.48 & 10.68 \\
\hline $\mathrm{Cl}$ & 0.33 & 0.21 & 0.33 & 0.21 & 0.33 & 0.21 & 0.33 & 0.17 & 0.33 & 0.16 & 0.33 & 0.24 & 0.33 & 0.22 & 0.33 & 0.22 & 0.33 & 0.22 \\
\hline $\mathrm{Cr}_{2} \mathrm{O}_{3}$ & 0.02 & 0.24 & 0.02 & 0.01 & 0.02 & 0.02 & 0.02 & 0.32 & 0.02 & 0.32 & 0.02 & 0.02 & 0.02 & 0.02 & 0.02 & 0.03 & 0.02 & 0.03 \\
\hline $\mathrm{F}$ & 0.03 & N/A & 0.03 & N/A & 0.03 & N/A & 0.03 & N/A & 0.03 & N/A & 0.03 & N/A & 0.03 & N/A & 0.03 & N/A & 0.03 & N/A \\
\hline $\mathrm{Fe}_{2} \mathrm{O}_{3}$ & 5.40 & 6.71 & 5.41 & 5.77 & 5.41 & 5.87 & 5.41 & 6.85 & 0.00 & 6.89 & 5.41 & 0.04 & 0.00 & 0.03 & 0.00 & 0.04 & 0.00 & 0.03 \\
\hline $\mathrm{K}_{2} \mathrm{O}$ & 0.26 & 0.32 & 2.76 & 2.71 & 2.76 & 2.68 & 2.76 & 2.59 & 2.76 & 2.55 & 2.76 & 2.77 & 2.76 & 2.67 & 2.76 & 2.59 & 2.76 & 2.59 \\
\hline $\mathrm{Li}_{2} \mathrm{O}$ & 2.50 & N/A & 0.00 & N/A & 0.00 & N/A & 0.00 & N/A & 0.00 & N/A & 0.00 & N/A & 0.00 & N/A & 0.00 & N/A & 0.00 & N/A \\
\hline $\mathrm{MgO}$ & 1.49 & 1.36 & 1.50 & 1.32 & 1.50 & 1.27 & 1.50 & 1.42 & 1.50 & 1.37 & 1.50 & 1.32 & 1.50 & 1.32 & 1.50 & 1.40 & 1.50 & 1.33 \\
\hline $\mathrm{Na}_{2} \mathrm{O}$ & 14.55 & 13.01 & 14.48 & 13.51 & 14.48 & 12.47 & 14.48 & 12.83 & 14.48 & 11.84 & 14.48 & 13.25 & 14.48 & 13.27 & 14.48 & 12.77 & 14.48 & 12.37 \\
\hline $\mathrm{P}_{2} \mathrm{O}_{5}$ & 0.13 & 0.16 & 0.13 & 0.17 & 0.13 & 0.16 & 0.13 & 0.16 & 0.13 & 0.17 & 0.13 & 0.17 & 0.13 & 0.17 & 0.13 & 0.17 & 0.13 & 0.18 \\
\hline $\mathrm{Re}_{2} \mathrm{O}_{7}$ & 0.00 & BDL & 0.10 & 0.03 & 0.10 & 0.03 & 0.10 & 0.02 & 0.10 & 0.02 & 0.10 & 0.03 & 0.10 & 0.02 & 0.10 & 0.02 & 0.10 & 0.03 \\
\hline $\mathrm{SiO}_{2}$ & 46.51 & 46.63 & 46.56 & 47.49 & 46.56 & 48.38 & 46.56 & 46.79 & 46.56 & 47.64 & 46.56 & 47.74 & 46.56 & 48.64 & 46.56 & 48.20 & 46.56 & 48.63 \\
\hline $\mathrm{SO}_{3}$ & 0.44 & $0.67^{\$}$ & 1.48 & 0.64 & 1.48 & 0.60 & 2.50 & 0.63 & 2.50 & 0.62 & 2.50 & 0.82 & 1.48 & 0.69 & 1.48 & 0.80 & 2.50 & 0.72 \\
\hline $\mathrm{TiO}_{2}$ & 1.14 & 1.30 & 1.14 & 1.24 & 1.14 & 1.27 & 1.14 & 1.30 & 1.14 & 1.33 & 1.14 & 1.31 & 1.14 & 1.26 & 1.14 & 1.32 & 1.14 & 1.31 \\
\hline $\mathrm{ZnO}$ & 3.06 & 3.27 & 3.06 & 3.06 & 3.06 & 3.05 & 3.06 & 3.21 & 3.06 & 3.22 & 3.06 & 3.01 & 3.06 & 2.82 & 3.06 & 3.07 & 3.06 & 3.06 \\
\hline $\mathrm{ZrO}_{2}$ & 3.02 & 3.77 & 3.02 & 3.51 & 3.02 & 3.65 & 3.02 & 3.62 & 3.02 & 3.81 & 3.02 & 3.53 & 3.02 & 3.45 & 3.02 & 3.55 & 3.02 & 3.67 \\
\hline Sum* & 100.00 & 100.80 & 101.10 & 100.60 & 101.10 & 100.60 & 102.20 & 100.60 & 102.20 & 100.52 & 102.20 & 100.78 & 101.10 & 100.75 & 101.10 & 100.81 & 102.20 & 100.77 \\
\hline
\end{tabular}

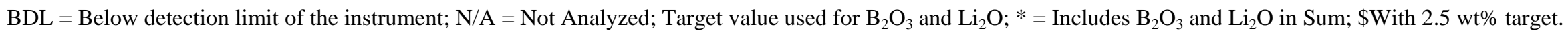


Table 4.6. XRF Analysis of Glasses Tested for LAW AN-104 (cont'd).

\begin{tabular}{|c|c|c|c|c|c|c|c|c|c|c|c|c|c|c|c|c|}
\hline \multirow{2}{*}{$\begin{array}{c}\text { Oxide } \\
- \\
\end{array}$} & \multicolumn{2}{|c|}{ LAWA120S1 } & \multicolumn{2}{|c|}{ LAWA120S1W } & \multicolumn{2}{|c|}{ LAWA120S2 } & \multicolumn{2}{|c|}{ LAWA120S2W } & \multicolumn{2}{|c|}{ LAWA121S1 } & \multicolumn{2}{|c|}{ LAWA121S1W } & \multicolumn{2}{|c|}{ LAWA121S2 } & \multicolumn{2}{|c|}{ LAWA121S2W } \\
\hline & Target & XRF & Target & XRF & Target & XRF & Target & XRF & Target & XRF & Target & XRF & Target & XRF & Target & XRF \\
\hline $\mathrm{Al}_{2} \mathrm{O}_{3}$ & 5.82 & 5.59 & 5.82 & 5.77 & 5.82 & 5.77 & 5.82 & 5.87 & 5.82 & 5.73 & 5.82 & 5.79 & 5.82 & 5.60 & 5.82 & 5.77 \\
\hline $\mathrm{B}_{2} \mathrm{O}_{3}$ & 9.31 & N/A & 9.31 & N/A & 9.31 & N/A & 9.31 & N/A & 9.31 & N/A & 9.31 & N/A & 9.31 & N/A & 9.31 & N/A \\
\hline $\mathrm{CaO}$ & 4.71 & 4.63 & 4.71 & 4.53 & 4.71 & 4.78 & 4.71 & 4.65 & 9.74 & 9.56 & 9.74 & 9.39 & 9.74 & 9.74 & 9.74 & 9.57 \\
\hline $\mathrm{Cl}$ & 0.33 & 0.22 & 0.33 & 0.21 & 0.33 & 0.18 & 0.33 & 0.18 & 0.33 & 0.23 & 0.33 & 0.22 & 0.33 & 0.18 & 0.33 & 0.17 \\
\hline $\mathrm{Cr}_{2} \mathrm{O}_{3}$ & 0.02 & 0.02 & 0.02 & 0.02 & 0.02 & 0.02 & 0.02 & 0.01 & 0.02 & 0.02 & 0.02 & 0.02 & 0.02 & 0.02 & 0.02 & 0.02 \\
\hline $\mathrm{F}$ & 0.03 & N/A & 0.03 & N/A & 0.03 & N/A & 0.03 & N/A & 0.03 & N/A & 0.03 & N/A & 0.03 & N/A & 0.03 & N/A \\
\hline $\mathrm{Fe}_{2} \mathrm{O}_{3}$ & 5.03 & 5.10 & 5.03 & 5.14 & 5.03 & 5.32 & 5.03 & 5.12 & 0.00 & 0.04 & 0.00 & 0.03 & 0.00 & 0.02 & 0.00 & 0.02 \\
\hline $\mathrm{K}_{2} \mathrm{O}$ & 8.31 & 7.58 & 8.31 & 7.46 & 8.31 & 7.57 & 8.31 & 7.39 & 8.31 & 7.71 & 8.31 & 7.54 & 8.31 & 7.42 & 8.31 & 7.29 \\
\hline $\mathrm{Li}_{2} \mathrm{O}$ & 0.00 & N/A & 0.00 & N/A & 0.00 & N/A & 0.00 & N/A & 0.00 & N/A & 0.00 & N/A & 0.00 & N/A & 0.00 & N/A \\
\hline $\mathrm{MgO}$ & 1.39 & 1.30 & 1.39 & 1.29 & 1.39 & 1.30 & 1.39 & 1.27 & 1.39 & 1.26 & 1.39 & 1.24 & 1.39 & 1.33 & 1.39 & 1.28 \\
\hline $\mathrm{Na}_{2} \mathrm{O}$ & 14.48 & 13.97 & 14.48 & 13.65 & 14.48 & 13.04 & 14.48 & 13.39 & 14.48 & 13.76 & 14.48 & 13.43 & 14.48 & 13.25 & 14.48 & 13.27 \\
\hline $\mathrm{P}_{2} \mathrm{O}_{5}$ & 0.13 & 0.17 & 0.13 & 0.17 & 0.13 & 0.16 & 0.13 & 0.12 & 0.13 & 0.16 & 0.13 & 0.16 & 0.13 & 0.16 & 0.13 & 0.17 \\
\hline $\mathrm{Re}_{2} \mathrm{O}_{7}$ & 0.10 & 0.01 & 0.10 & 0.01 & 0.10 & 0.01 & 0.01 & 0.02 & 0.10 & 0.02 & 0.10 & 0.02 & 0.10 & 0.01 & 0.10 & 0.01 \\
\hline $\mathrm{SiO}_{2}$ & 43.29 & 45.09 & 43.29 & 45.44 & 43.29 & 45.92 & 43.29 & 45.33 & 43.29 & 45.21 & 43.29 & 45.92 & 43.29 & 45.63 & 43.29 & 45.92 \\
\hline $\mathrm{SO}_{3}$ & 1.48 & 0.68 & 1.48 & 0.63 & 2.50 & 0.69 & 2.50 & 0.72 & 1.48 & 0.67 & 1.48 & 0.64 & 2.50 & 0.82 & 2.50 & 0.76 \\
\hline $\mathrm{TiO}_{2}$ & 1.06 & 1.13 & 1.06 & 1.13 & 1.06 & 1.16 & 1.06 & 1.15 & 1.06 & 1.12 & 1.06 & 1.10 & 1.06 & 1.14 & 1.06 & 1.13 \\
\hline $\mathrm{ZnO}$ & 2.85 & 2.69 & 2.85 & 2.66 & 2.85 & 2.82 & 2.85 & 2.76 & 2.85 & 2.73 & 2.85 & 2.67 & 2.85 & 2.76 & 2.85 & 2.72 \\
\hline $\mathrm{ZrO}_{2}$ & 2.81 & 3.03 & 2.81 & 3.09 & 2.81 & 3.25 & 2.81 & 3.23 & 2.81 & 3.05 & 2.81 & 3.07 & 2.81 & 3.21 & 2.81 & 3.20 \\
\hline Sum* & 101.10 & 100.50 & 101.10 & 100.50 & 102.20 & 101.30 & 102.20 & 100.52 & 101.10 & 100.56 & 101.10 & 100.55 & 102.20 & 100.59 & 102.20 & 100.60 \\
\hline
\end{tabular}

$\mathrm{BDL}=$ Below detection limit of the instrument; N/A = Not Analyzed; Target value used for $\mathrm{B}_{2} \mathrm{O}_{3}$ and $\mathrm{Li}_{2} \mathrm{O}$; * = Includes $\mathrm{B}_{2} \mathrm{O}_{3}$ and $\mathrm{Li} \mathrm{I}_{2} \mathrm{O}$ in $\mathrm{Sum}$ 
Table 4.6. XRF Analysis of Glasses Tested for LAW AN-104 (cont'd).

\begin{tabular}{|c|c|c|c|c|c|c|c|c|c|c|}
\hline \multirow{2}{*}{$\begin{array}{r}\text { Oxide } \\
- \\
\end{array}$} & \multicolumn{2}{|c|}{ LAWA122S2W } & \multicolumn{2}{|c|}{ LAWA123S1 } & \multicolumn{2}{|c|}{ LAWA123S1W } & \multicolumn{2}{|c|}{ LAWA123S2 } & \multicolumn{2}{|c|}{ LAWA123S2W } \\
\hline & Target & XRF & Target & XRF & Target & XRF & Target & XRF & Target & XRF \\
\hline $\mathrm{Al}_{2} \mathrm{O}_{3}$ & 5.93 & 5.73 & 5.93 & 5.70 & 5.93 & 5.82 & 5.93 & 5.91 & 5.93 & 6.08 \\
\hline $\mathrm{B}_{2} \mathrm{O}_{3}$ & 9.64 & N/A & 9.64 & N/A & 9.64 & N/A & 9.64 & N/A & 9.64 & N/A \\
\hline $\mathrm{CaO}$ & 4.88 & 4.96 & 10.10 & 10.19 & 10.10 & 9.78 & 10.10 & 10.09 & 10.10 & 9.77 \\
\hline $\mathrm{Cl}$ & 0.33 & 0.16 & 0.33 & 0.23 & 0.33 & 0.22 & 0.33 & 0.17 & 0.33 & 0.16 \\
\hline $\mathrm{Cr}_{2} \mathrm{O}_{3}$ & 0.02 & 0.03 & 0.02 & 0.01 & 0.02 & 0.01 & 0.02 & 0.02 & 0.02 & 0.02 \\
\hline $\mathrm{F}$ & 0.03 & N/A & 0.03 & N/A & 0.03 & N/A & 0.03 & N/A & 0.03 & N/A \\
\hline $\mathrm{Fe}_{2} \mathrm{O}_{3}$ & 5.21 & 5.69 & 0.00 & 0.03 & 0.00 & 0.02 & 0.00 & 0.03 & 0.00 & 0.02 \\
\hline $\mathrm{K}_{2} \mathrm{O}$ & 5.64 & 5.10 & 5.64 & 5.40 & 5.64 & 5.22 & 5.64 & 5.09 & 5.64 & 4.97 \\
\hline $\mathrm{Li}_{2} \mathrm{O}$ & 0.00 & N/A & 0.00 & N/A & 0.00 & N/A & 0.00 & N/A & 0.00 & N/A \\
\hline $\mathrm{MgO}$ & 1.44 & 1.26 & 1.44 & 1.27 & 1.44 & 1.32 & 1.44 & 1.39 & 1.44 & 1.33 \\
\hline $\mathrm{Na}_{2} \mathrm{O}$ & 14.48 & 12.69 & 14.48 & 13.21 & 14.48 & 13.34 & 14.48 & 13.32 & 14.48 & 12.82 \\
\hline $\mathrm{P}_{2} \mathrm{O}_{5}$ & 0.13 & 0.16 & 0.13 & 0.17 & 0.13 & 0.18 & 0.13 & 0.16 & 0.13 & 0.17 \\
\hline $\mathrm{Re}_{2} \mathrm{O}_{7}$ & 0.10 & 0.02 & 0.10 & 0.02 & 0.10 & 0.02 & 0.10 & 0.01 & 0.10 & 0.01 \\
\hline $\mathrm{SiO}_{2}$ & 44.87 & 46.69 & 44.87 & 46.57 & 44.87 & 47.11 & 44.87 & 46.70 & 44.87 & 47.77 \\
\hline $\mathrm{SO}_{3}$ & 2.50 & 0.67 & 1.48 & 0.73 & 1.48 & 0.69 & 2.50 & 0.78 & 2.50 & 0.76 \\
\hline $\mathrm{TiO}_{2}$ & 1.10 & 1.22 & 1.10 & 1.21 & 1.10 & 1.19 & 1.10 & 1.19 & 1.10 & 1.17 \\
\hline $\mathrm{ZnO}$ & 2.95 & 3.01 & 2.95 & 2.88 & 2.95 & 2.78 & 2.95 & 2.89 & 2.95 & 2.78 \\
\hline $\mathrm{ZrO}_{2}$ & 2.91 & 3.55 & 2.91 & 3.41 & 2.91 & 3.31 & 2.91 & 3.31 & 2.91 & 3.21 \\
\hline Sum* & 102.16 & 100.58 & 101.15 & 100.66 & 101.15 & 100.64 & 102.17 & 100.70 & 102.17 & 100.68 \\
\hline
\end{tabular}

$\mathrm{BDL}=$ Below detection limit of the instrument; N/A = Not Analyzed; Target value used for $\mathrm{B}_{2} \mathrm{O}_{3}$ and $\mathrm{Li}_{2} \mathrm{O}$; * = Includes $\mathrm{B}_{2} \mathrm{O}_{3}$ and $\mathrm{Li}_{2} \mathrm{O}$ in $\mathrm{Sum}$ 
Table 4.7. XRF Analysis of Glasses Tested for LAW AZ-101.

\begin{tabular}{|c|c|c|c|c|c|c|c|c|c|c|c|c|c|c|c|c|}
\hline \multirow{2}{*}{$\begin{array}{c}\text { Oxide } \\
- \\
\end{array}$} & \multicolumn{2}{|c|}{ LAWB45S0 } & \multicolumn{2}{|c|}{ LAWB45S0R2 } & \multicolumn{2}{|c|}{ LAWB45S4X } & \multicolumn{2}{|c|}{ LAWB60S0 } & \multicolumn{2}{|c|}{ LAWB60S2 } & \multicolumn{2}{|c|}{ LAWB60S2W } & \multicolumn{2}{|c|}{ LAWB60S4 } & \multicolumn{2}{|c|}{ LAWB60S4W } \\
\hline & Target & $\mathrm{XRF}$ & Target & XRF & Target & $\mathrm{XRF}$ & Target & $\mathrm{XRF}$ & Target & $\mathrm{XRF}$ & Target & $\mathrm{XRF}$ & Target & $\mathrm{XRF}$ & Target & XRF \\
\hline $\mathrm{Al}_{2} \mathrm{O}_{3}$ & 6.13 & 6.14 & 6.13 & 6.31 & 6.13 & 5.96 & 6.13 & 6.34 & 6.13 & 6.02 & 6.13 & 6.04 & 6.13 & 6.29 & 6.13 & 6.28 \\
\hline $\mathrm{B}_{2} \mathrm{O}_{3}$ & 12.34 & N/A & 12.34 & N/A & 12.34 & $\mathrm{~N} / \mathrm{A}$ & 12.34 & N/A & 12.34 & N/A & 12.34 & N/A & 12.34 & N/A & 12.34 & N/A \\
\hline $\mathrm{CaO}$ & 6.63 & 6.78 & 6.63 & 6.74 & 6.63 & 6.13 & 11.88 & 12.01 & 11.88 & 11.44 & 11.88 & 11.43 & 11.88 & 11.67 & 11.88 & 11.39 \\
\hline $\mathrm{Cl}$ & 0.01 & 0.02 & 0.01 & 0.01 & 0.01 & BDL & 0.01 & BDL & 0.01 & 0.01 & 0.01 & BDL & 0.01 & 0.00 & 0.01 & BDL \\
\hline $\mathrm{Cr}_{2} \mathrm{O}_{3}$ & 0.07 & 0.07 & 0.07 & 0.08 & 0.07 & 0.06 & 0.07 & 0.07 & 0.07 & 0.07 & 0.07 & 0.06 & 0.07 & 0.07 & 0.07 & 0.06 \\
\hline $\mathrm{Cs}_{2} \mathrm{O}$ & 0.00 & 0.00 & 0.00 & BDL & 0.00 & BDL & 0.00 & 0.01 & 0.00 & 0.01 & 0.00 & 0.01 & 0.00 & 0.01 & 0.00 & 0.02 \\
\hline $\mathrm{F}$ & 0.08 & N/A & 0.08 & N/A & 0.08 & N/A & 0.08 & N/A & 0.08 & N/A & 0.08 & N/A & 0.08 & N/A & 0.08 & N/A \\
\hline $\mathrm{Fe}_{2} \mathrm{O}_{3}$ & 5.26 & 5.62 & 5.26 & 5.74 & 5.26 & 5.23 & 0.00 & 0.03 & 0.00 & 0.03 & 0.00 & 0.03 & 0.00 & 0.03 & 0.00 & 0.02 \\
\hline $\mathrm{K}_{2} \mathrm{O}$ & 0.26 & 0.38 & 0.26 & 0.38 & 0.26 & 0.30 & 0.26 & 0.35 & 0.26 & 0.33 & 0.26 & 0.33 & 0.26 & 0.30 & 0.26 & 0.29 \\
\hline $\mathrm{Li}_{2} \mathrm{O}$ & 4.62 & N/A & 4.62 & N/A & 4.62 & N/A & 4.62 & N/A & 4.62 & N/A & 4.62 & N/A & 4.62 & N/A & 4.62 & N/A \\
\hline $\mathrm{MgO}$ & 2.97 & 2.82 & 2.97 & 2.82 & 2.97 & 2.89 & 2.97 & 2.72 & 2.97 & 2.79 & 2.97 & 2.75 & 2.97 & 2.75 & 2.97 & 2.74 \\
\hline $\mathrm{Na}_{2} \mathrm{O}$ & 6.50 & 6.33 & 6.50 & 6.52 & $6.50+2.00$ & 7.27 & 6.50 & 6.66 & 6.50 & 6.42 & 6.50 & 5.95 & 6.50 & 5.47 & 6.50 & 5.33 \\
\hline $\mathrm{P}_{2} \mathrm{O}_{5}$ & 0.03 & 0.05 & 0.03 & 0.04 & 0.03 & 0.04 & 0.03 & 0.05 & 0.03 & 0.05 & 0.03 & 0.04 & 0.03 & 0.05 & 0.03 & 0.05 \\
\hline $\mathrm{Re}_{2} \mathrm{O}_{7}$ & 0.10 & $\mathrm{BDL}$ & 0.10 & 0.09 & 0.10 & 0.02 & 0.10 & 0.08 & 0.10 & 0.04 & 0.10 & 0.03 & 0.10 & 0.02 & 0.10 & 0.03 \\
\hline $\mathrm{SiO}_{2}$ & 47.86 & 49.13 & 47.86 & 48.71 & 47.86 & 48.84 & 47.86 & 49.14 & 47.86 & 49.52 & 47.86 & 49.93 & 47.86 & 49.43 & 47.86 & 50.03 \\
\hline $\mathrm{SO}_{3}$ & 0.00 & 0.01 & 0.00 & 0.02 & 1.40 & 1.35 & 0.00 & - & 2.50 & 1.46 & 2.50 & 1.41 & 4.50 & 1.62 & 4.50 & 1.60 \\
\hline $\mathrm{TiO}_{2}$ & 0.00 & 0.20 & 0.00 & 0.02 & 0.00 & 0.01 & 0.00 & 0.02 & 0.00 & 0.00 & 0.00 & 0.01 & 0.00 & 0.02 & 0.00 & 0.02 \\
\hline $\mathrm{ZnO}$ & 3.15 & 2.97 & 3.15 & 3.28 & 3.15 & 2.91 & 3.15 & 3.05 & 3.15 & 2.85 & 3.15 & 2.88 & 3.15 & 3.07 & 3.15 & 2.94 \\
\hline $\mathrm{ZrO}_{2}$ & 3.15 & 3.65 & 3.15 & 3.45 & 3.15 & 3.38 & 3.15 & 3.71 & 3.15 & 3.37 & 3.15 & 3.47 & 3.15 & 3.64 & 3.15 & 3.62 \\
\hline Sum* & 99.16 & 101.12 & 99.16 & 101.14 & 94.06 & 101.33 & 103.66 & 101.19 & 103.66 & 101.36 & 99.16 & 101.32 & 101.66 & 101.39 & 101.66 & 101.36 \\
\hline
\end{tabular}

$\mathrm{BDL}=$ Below detection limit of the instrument; N/A = Not Analyzed; Target value used for $\mathrm{B}_{2} \mathrm{O}_{3}$ and $\mathrm{Li}_{2} \mathrm{O}$; $*=\mathrm{Includes}_{2} \mathrm{O}_{3}$ and $\mathrm{Li}_{2} \mathrm{O}$ in $\mathrm{Sum}$ 
Table 4.7. XRF Analysis of Glasses Tested for LAW AZ-101 (cont'd).

\begin{tabular}{|c|c|c|c|c|c|c|c|c|c|c|c|c|}
\hline Oxide & \multicolumn{2}{|c|}{ LAWB69 } & \multicolumn{2}{|c|}{ LAWB69S4 } & \multicolumn{2}{|c|}{ LAWB69S4W } & \multicolumn{2}{|c|}{ LAWB69S0 } & \multicolumn{2}{|c|}{ LAWB70 } & \multicolumn{2}{|c|}{ LAWB70S0 } \\
\hline- & Target & XRF & Target & XRF & Target & XRF & Target & XRF & Target & XRF & Target & XRF \\
\hline $\mathrm{Al}_{2} \mathrm{O}_{3}$ & 6.15 & 6.03 & 6.15 & 6.14 & 6.15 & 6.45 & 6.15 & 6.38 & 6.15 & 6.19 & 6.15 & 6.31 \\
\hline $\mathrm{B}_{2} \mathrm{O}_{3}$ & 12.33 & N/A & 12.33 & $\mathrm{~N} / \mathrm{A}$ & 12.33 & N/A & 12.33 & N/A & 12.33 & N/A & 12.33 & N/A \\
\hline $\mathrm{CaO}$ & 10.46 & 10.31 & 10.46 & 10.04 & 10.46 & 10.20 & 10.46 & 10.58 & 6.62 & 6.44 & 6.62 & 6.65 \\
\hline $\mathrm{Cl}$ & 0.01 & 0.01 & 0.01 & $\mathrm{BDL}$ & 0.01 & BDL & 0.01 & $\mathrm{BDL}$ & 0.01 & $\mathrm{BDL}$ & 0.01 & 0.01 \\
\hline $\mathrm{Cr}_{2} \mathrm{O}_{3}$ & 0.05 & 0.05 & 0.05 & 0.05 & 0.05 & 0.05 & 0.05 & 0.05 & 0.05 & 0.05 & 0.05 & 0.04 \\
\hline $\mathrm{F}$ & 0.08 & N/A & 0.08 & N/A & 0.08 & N/A & 0.08 & N/A & 0.08 & N/A & 0.08 & N/A \\
\hline $\mathrm{Fe}_{2} \mathrm{O}_{3}$ & 0.00 & 0.05 & 0.00 & 0.06 & 0.00 & 0.06 & 0.00 & 0.05 & 3.25 & 3.27 & 3.25 & 3.39 \\
\hline $\mathrm{K}_{2} \mathrm{O}$ & 0.23 & 0.29 & 0.23 & 0.27 & 0.23 & 0.27 & 0.23 & 0.32 & 0.23 & 0.30 & 0.23 & 0.30 \\
\hline $\mathrm{Li}_{2} \mathrm{O}$ & 4.61 & N/A & 4.61 & N/A & 4.61 & N/A & 4.61 & N/A & 4.61 & N/A & 4.61 & N/A \\
\hline $\mathrm{MgO}$ & 2.97 & 2.93 & 2.97 & 2.94 & 2.97 & 3.01 & 2.97 & 2.68 & 2.97 & 3.00 & 2.97 & 2.83 \\
\hline $\mathrm{Na}_{2} \mathrm{O}$ & 6.62 & 6.79 & 6.62 & 6.23 & 6.62 & 5.79 & 6.62 & 6.60 & 6.62 & 7.13 & 6.62 & 6.98 \\
\hline $\mathrm{P}_{2} \mathrm{O}_{5}$ & 0.05 & 0.06 & 0.05 & 0.07 & 0.05 & 0.06 & 0.05 & 0.06 & 0.05 & 0.06 & 0.05 & 0.06 \\
\hline $\mathrm{Re}_{2} \mathrm{O}_{7}$ & 0.10 & 0.05 & 0.10 & 0.01 & 0.10 & 0.02 & 0.10 & 0.06 & 0.10 & 0.05 & 0.10 & 0.06 \\
\hline $\mathrm{SiO}_{2}$ & 47.95 & 49.21 & 47.95 & 49.30 & 47.95 & 48.94 & 47.95 & 49.69 & 47.95 & 49.21 & 47.95 & 49.15 \\
\hline $\mathrm{SO}_{3}$ & 0.78 & 0.65 & 4.50 & 1.36 & 4.50 & 1.19 & 0.00 & 0.01 & 0.78 & 0.54 & 0.00 & 0.03 \\
\hline $\mathrm{TiO}_{2}$ & 0.00 & 0.01 & 0.00 & 0.02 & 0.00 & 0.02 & 0.00 & 0.02 & 0.00 & 0.01 & 0.00 & 0.02 \\
\hline $\mathrm{ZnO}$ & 4.57 & 4.37 & 4.57 & 4.46 & 4.57 & 4.63 & 4.57 & 4.39 & 5.15 & 4.79 & 5.15 & 4.98 \\
\hline $\mathrm{ZrO}_{2}$ & 3.15 & 3.61 & 3.15 & 3.56 & 3.15 & 3.88 & 3.15 & 3.41 & 3.15 & 3.38 & 3.15 & 3.53 \\
\hline Sum* & 100.10 & 101.30 & 103.80 & 101.40 & 103.80 & 101.50 & 99.30 & 101.20 & 100.10 & 101.40 & 99.30 & 101.30 \\
\hline
\end{tabular}

$\mathrm{BDL}=$ Below detection limit of the instrument; N/A = Not Analyzed; Target value used for $\mathrm{B}_{2} \mathrm{O}_{3}$ and $\mathrm{Li}_{2} \mathrm{O}$; * $=$ Includes $\mathrm{B}_{2} \mathrm{O}_{3}$ and $\mathrm{Li}_{2} \mathrm{O}$ in Sum 
Table 4.7. XRF Analysis of Glasses Tested for LAW AZ-101 (cont'd).

\begin{tabular}{|c|c|c|c|c|c|c|c|c|c|c|c|c|c|c|c|c|}
\hline \multirow{2}{*}{$\begin{array}{c}\text { Oxide } \\
- \\
\end{array}$} & \multicolumn{2}{|c|}{ LAWB70S4 } & \multicolumn{2}{|c|}{ LAWB70S4W } & \multicolumn{2}{|c|}{ LAWB71 } & \multicolumn{2}{|c|}{ LAWB71S0 } & \multicolumn{2}{|c|}{ LAWB71S4 } & \multicolumn{2}{|c|}{ LAWB71S4W } & \multicolumn{2}{|c|}{ LAWB72 } & \multicolumn{2}{|c|}{ LAWB72S0 } \\
\hline & Target & XRF & Target & XRF & Target & XRF & Target & XRF & Target & XRF & Target & XRF & Target & XRF & Target & XRF \\
\hline $\mathrm{Al}_{2} \mathrm{O}_{3}$ & 6.15 & 6.18 & 6.15 & 6.09 & 6.15 & 6.02 & 6.15 & 6.31 & 6.15 & 6.34 & 6.15 & 6.30 & 6.15 & 5.63 & 6.15 & 6.19 \\
\hline $\mathrm{B}_{2} \mathrm{O}_{3}$ & 12.33 & N/A & 12.33 & N/A & 10.78 & N/A & 10.78 & N/A & 10.78 & N/A & 10.78 & N/A & 12.33 & N/A & 12.33 & N/A \\
\hline $\mathrm{CaO}$ & 6.62 & 6.19 & 6.62 & 6.04 & 6.62 & 6.63 & 6.62 & 6.68 & 6.62 & 6.39 & 6.62 & 6.49 & 7.12 & 7.19 & 7.12 & 7.11 \\
\hline $\mathrm{Cl}$ & 0.01 & $\mathrm{BDL}$ & 0.01 & $\mathrm{BDL}$ & 0.01 & 0.01 & 0.01 & 0.00 & 0.01 & $\mathrm{BDL}$ & 0.01 & $\mathrm{BDL}$ & 0.01 & 0.01 & 0.01 & $\mathrm{BDL}$ \\
\hline $\mathrm{Cr}_{2} \mathrm{O}_{3}$ & 0.05 & 0.05 & 0.05 & 0.05 & 0.05 & 0.06 & 0.05 & 0.05 & 0.05 & 0.04 & 0.05 & 0.05 & 0.05 & 0.29 & 0.05 & 0.05 \\
\hline $\mathrm{F}$ & 0.08 & N/A & 0.08 & N/A & 0.08 & N/A & 0.08 & N/A & 0.08 & N/A & 0.08 & N/A & 0.08 & N/A & 0.08 & N/A \\
\hline $\mathrm{Fe}_{2} \mathrm{O}_{3}$ & 3.25 & 3.36 & 3.25 & 3.32 & 3.25 & 3.42 & 3.25 & 3.46 & 3.25 & 3.27 & 3.25 & 3.47 & 3.25 & 4.09 & 3.25 & 3.35 \\
\hline $\mathrm{K}_{2} \mathrm{O}$ & 0.23 & 0.27 & 0.23 & 0.26 & 0.23 & 0.30 & 0.23 & 0.30 & 0.23 & 0.27 & 0.23 & 0.28 & 0.23 & 0.29 & 0.23 & 0.30 \\
\hline $\mathrm{Li}_{2} \mathrm{O}$ & 4.61 & N/A & 4.61 & N/A & 4.61 & N/A & 4.61 & N/A & 4.61 & N/A & 4.61 & N/A & 4.11 & N/A & 4.11 & N/A \\
\hline MgO & 2.97 & 2.79 & 2.97 & 2.82 & 2.97 & 2.85 & 2.97 & 2.93 & 2.97 & 2.93 & 2.97 & 2.81 & 2.97 & 2.85 & 2.97 & 3.02 \\
\hline $\mathrm{Na}_{2} \mathrm{O}$ & 6.62 & 5.85 & 6.62 & 5.92 & 6.62 & 6.90 & 6.62 & 6.60 & 6.62 & 6.41 & 6.62 & 5.91 & 6.62 & 6.39 & 6.62 & 6.92 \\
\hline $\mathrm{P}_{2} \mathrm{O}_{5}$ & 0.05 & 0.06 & 0.05 & 0.06 & 0.05 & 0.06 & 0.05 & 0.07 & 0.05 & 0.06 & 0.05 & 0.06 & 0.05 & 0.06 & 0.05 & 0.06 \\
\hline $\mathrm{Re}_{2} \mathrm{O}_{7}$ & 0.10 & 0.03 & 0.10 & 0.02 & 0.10 & 0.07 & 0.10 & 0.07 & 0.10 & 0.03 & 0.10 & 0.04 & 0.10 & 0.05 & 0.10 & 0.04 \\
\hline $\mathrm{SiO}_{2}$ & 47.95 & 48.54 & 47.95 & 48.90 & 47.95 & 48.90 & 47.95 & 48.92 & 47.95 & 50.64 & 47.95 & 50.51 & 47.95 & 48.27 & 47.95 & 49.21 \\
\hline $\mathrm{SO}_{3}$ & 4.50 & 1.26 & 4.50 & 1.23 & 0.78 & 0.48 & 0.00 & 0.02 & 4.50 & 1.30 & 4.50 & 1.26 & 0.78 & 0.61 & 0.00 & 0.01 \\
\hline $\mathrm{TiO}_{2}$ & 0.00 & 1.58 & 0.00 & 1.55 & 1.55 & 1.61 & 1.55 & 1.65 & 1.55 & 0.05 & 1.55 & 0.03 & 0.00 & 0.02 & 0.00 & 0.05 \\
\hline $\mathrm{ZnO}$ & 5.15 & 4.84 & 5.15 & 4.76 & 5.15 & 5.01 & 5.15 & 5.09 & 5.15 & 4.83 & 5.15 & 5.08 & 5.15 & 5.29 & 5.15 & 4.98 \\
\hline $\mathrm{ZrO}_{2}$ & 3.15 & 3.42 & 3.15 & 3.41 & 3.15 & 3.51 & 3.15 & 3.63 & 3.15 & 3.40 & 3.15 & 3.68 & 3.15 & 3.72 & 3.15 & 3.55 \\
\hline Sum* & 103.80 & 101.40 & 103.80 & 101.40 & 100.10 & 101.20 & 99.30 & 101.20 & 103.80 & 101.30 & 103.80 & 101.30 & 100.10 & 101.20 & 99.30 & 101.30 \\
\hline
\end{tabular}


Table 4.7. XRF Analysis of Glasses Tested for LAW AZ-101 (cont'd).

\begin{tabular}{|c|c|c|c|c|c|c|c|c|c|c|c|c|c|c|c|c|}
\hline \multirow{2}{*}{$\begin{array}{c}\text { Oxide } \\
- \\
\end{array}$} & \multicolumn{2}{|c|}{ LAWB72S4 } & \multicolumn{2}{|c|}{ LAWB72S4W } & \multicolumn{2}{|c|}{ LAWB77 } & \multicolumn{2}{|c|}{ LAWB77S4 } & \multicolumn{2}{|c|}{ LAWB77S4W } & \multicolumn{2}{|c|}{ LAWB77S0 } & \multicolumn{2}{|c|}{ LAWB78 } & \multicolumn{2}{|c|}{ LAWB78S0 } \\
\hline & Target & $\mathrm{XRF}$ & Target & XRF & Target & XRF & Target & XRF & Target & $\mathrm{XRF}$ & Target & $\mathrm{XRF}$ & Target & $\mathrm{XRF}$ & Target & $\mathrm{XRF}$ \\
\hline $\mathrm{Al}_{2} \mathrm{O}_{3}$ & 6.15 & 6.21 & 6.15 & 6.13 & 6.15 & 5.70 & 6.15 & 6.34 & 6.15 & 6.15 & 6.15 & 6.11 & 6.15 & 5.68 & 6.15 & 6.42 \\
\hline $\mathrm{B}_{2} \mathrm{O}_{3}$ & 12.33 & N/A & 12.33 & N/A & 12.33 & N/A & 12.33 & N/A & 12.33 & N/A & 12.33 & N/A & 12.33 & N/A & 12.33 & N/A \\
\hline $\mathrm{CaO}$ & 7.12 & 6.75 & 7.12 & 6.69 & 6.62 & 6.74 & 6.62 & 6.39 & 6.62 & 6.34 & 6.62 & 6.77 & 7.12 & 7.31 & 7.12 & 7.13 \\
\hline $\mathrm{Cl}$ & 0.01 & $\mathrm{BDL}$ & 0.01 & $\mathrm{BDL}$ & 0.01 & 0.00 & 0.01 & 0.01 & 0.01 & 0.00 & 0.01 & $\mathrm{BDL}$ & 0.01 & $\mathrm{BDL}$ & 0.01 & 0.01 \\
\hline $\mathrm{Cr}_{2} \mathrm{O}_{3}$ & 0.05 & 0.04 & 0.05 & 0.04 & 0.05 & 0.22 & 0.05 & 0.05 & 0.05 & 0.05 & 0.05 & 0.05 & 0.05 & 0.23 & 0.05 & 0.05 \\
\hline $\mathrm{F}$ & 0.08 & N/A & 0.08 & N/A & 0.08 & N/A & 0.08 & N/A & 0.08 & N/A & 0.08 & N/A & 0.08 & N/A & 0.08 & N/A \\
\hline $\mathrm{Fe}_{2} \mathrm{O}_{3}$ & 3.25 & 3.34 & 3.25 & 3.39 & 2.20 & 2.84 & 2.20 & 2.33 & 2.20 & 2.39 & 2.20 & 2.38 & 3.25 & 3.96 & 3.25 & 3.36 \\
\hline $\mathrm{K}_{2} \mathrm{O}$ & 0.23 & 0.29 & 0.23 & 0.27 & 0.23 & 0.29 & 0.23 & 0.28 & 0.23 & 0.27 & 0.23 & 0.30 & 0.23 & 0.28 & 0.23 & 0.31 \\
\hline $\mathrm{Li}_{2} \mathrm{O}$ & 4.11 & N/A & 4.11 & N/A & 4.11 & N/A & 4.11 & N/A & 4.11 & N/A & 4.11 & N/A & 3.05 & N/A & 3.05 & N/A \\
\hline $\mathrm{MgO}$ & 2.97 & 2.82 & 2.97 & 2.75 & 2.97 & 2.82 & 2.97 & 2.81 & 2.97 & 2.65 & 2.97 & 2.81 & 2.97 & 2.79 & 2.97 & 2.92 \\
\hline $\mathrm{Na}_{2} \mathrm{O}$ & 6.62 & 6.07 & 6.62 & 5.58 & 6.62 & 7.05 & 6.62 & 6.12 & 6.62 & 5.62 & 6.62 & 6.72 & 9.78 & 10.08 & 9.78 & 10.08 \\
\hline $\mathrm{P}_{2} \mathrm{O}_{5}$ & 0.05 & 0.07 & 0.05 & 0.06 & 0.05 & 0.06 & 0.05 & 0.06 & 0.05 & 0.07 & 0.05 & 0.06 & 0.05 & 0.05 & 0.05 & 0.06 \\
\hline $\mathrm{Re}_{2} \mathrm{O}_{7}$ & 0.10 & 0.02 & 0.10 & 0.03 & 0.10 & 0.05 & 0.10 & 0.03 & 0.10 & 0.03 & 0.10 & 0.08 & 0.10 & 0.06 & 0.10 & 0.08 \\
\hline $\mathrm{SiO}_{2}$ & 47.95 & 49.15 & 47.95 & 50.08 & 47.95 & 47.95 & 47.95 & 49.60 & 47.95 & 50.10 & 47.95 & 49.47 & 47.00 & 47.15 & 47.00 & 48.09 \\
\hline $\mathrm{SO}_{3}$ & 4.50 & 1.30 & 4.50 & 1.20 & 0.78 & 0.52 & 4.50 & 1.22 & 4.50 & 1.17 & 0.00 & 0.01 & 0.78 & 0.51 & 0.00 & 0.01 \\
\hline $\mathrm{TiO}_{2}$ & 0.00 & 0.02 & 0.00 & 0.02 & 1.55 & 1.63 & 1.55 & 1.60 & 1.55 & 1.62 & 1.55 & 1.63 & 0.00 & 0.01 & 0.00 & 0.02 \\
\hline $\mathrm{ZnO}$ & 5.15 & 4.93 & 5.15 & 4.86 & 5.15 & 5.26 & 5.15 & 4.88 & 5.15 & 4.94 & 5.15 & 5.06 & 4.00 & 4.10 & 4.00 & 3.84 \\
\hline $\mathrm{ZrO}_{2}$ & 3.15 & 3.96 & 3.15 & 3.79 & 3.15 & 3.62 & 3.15 & 3.20 & 3.15 & 3.43 & 3.15 & 3.28 & 3.15 & 3.60 & 3.15 & 3.53 \\
\hline Sum* & 103.80 & 101.40 & 103.80 & 101.30 & 100.10 & 101.20 & 103.80 & 101.30 & 103.80 & 101.30 & 99.30 & 101.20 & 100.10 & 101.20 & 99.30 & 101.30 \\
\hline
\end{tabular}


Table 4.7. XRF Analysis of Glasses Tested for LAW AZ-101 (cont'd).

\begin{tabular}{|c|c|c|c|c|c|c|c|c|c|c|c|c|c|c|c|c|}
\hline Oxide & \multicolumn{2}{|c|}{ LAWB78S4 } & \multicolumn{2}{|c|}{ LAWB78S4W } & \multicolumn{2}{|c|}{ LAWB79 } & \multicolumn{2}{|c|}{ LAWB79S0 } & \multicolumn{2}{|c|}{ LAWB79S4 } & \multicolumn{2}{|c|}{ LAWB79S4W } & \multicolumn{2}{|c|}{ LAWB80 } & \multicolumn{2}{|c|}{ LAWB80S0 } \\
\hline- & Target & XRF & Target & XRF & Target & XRF & Target & XRF & Target & XRF & Target & XRF & Target & XRF & Target & XRF \\
\hline $\mathrm{Al}_{2} \mathrm{O}_{3}$ & 6.15 & 6.36 & 6.15 & 6.28 & 6.15 & 5.82 & 6.15 & 6.36 & 6.15 & 6.68 & 6.15 & 6.45 & 6.15 & 6.15 & 6.15 & 6.37 \\
\hline $\mathrm{B}_{2} \mathrm{O}_{3}$ & 12.33 & N/A & 12.33 & N/A & 12.33 & N/A & 12.33 & N/A & 12.33 & N/A & 12.33 & N/A & 12.33 & N/A & 12.33 & N/A \\
\hline $\mathrm{CaO}$ & 7.12 & 6.92 & 7.12 & 6.81 & 7.12 & 7.34 & 7.12 & 7.13 & 7.12 & 6.63 & 7.12 & 6.63 & 7.12 & 7.29 & 7.12 & 7.13 \\
\hline $\mathrm{Cl}$ & 0.01 & $\mathrm{BDL}$ & 0.01 & $\mathrm{BDL}$ & 0.01 & $\mathrm{BDL}$ & 0.01 & BDL & 0.01 & $\mathrm{BDL}$ & 0.01 & 0.01 & 0.01 & $\mathrm{BDL}$ & 0.01 & BDL \\
\hline $\mathrm{Cr}_{2} \mathrm{O}_{3}$ & 0.05 & 0.04 & 0.05 & 0.05 & 0.05 & 0.23 & 0.05 & 0.04 & 0.05 & 0.04 & 0.05 & 0.04 & 0.05 & 0.05 & 0.05 & 0.05 \\
\hline $\mathrm{F}$ & 0.08 & N/A & 0.08 & N/A & 0.08 & N/A & 0.08 & N/A & 0.08 & N/A & 0.08 & N/A & 0.08 & N/A & 0.08 & N/A \\
\hline $\mathrm{Fe}_{2} \mathrm{O}_{3}$ & 3.25 & 3.40 & 3.25 & 3.38 & 3.25 & 3.92 & 3.25 & 3.35 & 3.25 & 3.22 & 3.25 & 3.28 & 3.25 & 3.44 & 3.25 & 3.37 \\
\hline $\mathrm{K}_{2} \mathrm{O}$ & 0.23 & 0.27 & 0.23 & 0.26 & 0.23 & 0.29 & 0.23 & 0.31 & 0.23 & 0.30 & 0.23 & 0.27 & 1.99 & 1.89 & 1.99 & 1.86 \\
\hline $\mathrm{Li}_{2} \mathrm{O}$ & 3.05 & N/A & 3.05 & N/A & 3.51 & N/A & 3.51 & N/A & 3.51 & N/A & 3.51 & N/A & 3.51 & N/A & 3.51 & N/A \\
\hline $\mathrm{MgO}$ & 2.97 & 2.89 & 2.97 & 2.73 & 2.97 & 2.92 & 2.97 & 2.92 & 2.97 & 2.98 & 2.97 & 2.84 & 2.97 & 2.82 & 2.97 & 2.96 \\
\hline $\mathrm{Na}_{2} \mathrm{O}$ & 9.78 & 8.90 & 9.78 & 8.25 & 8.62 & 8.75 & 8.62 & 8.87 & 8.62 & 7.77 & 8.62 & 7.54 & 6.62 & 6.25 & 6.62 & 6.89 \\
\hline $\mathrm{P}_{2} \mathrm{O}_{5}$ & 0.05 & 0.07 & 0.05 & 0.07 & 0.05 & 0.05 & 0.05 & 0.06 & 0.05 & 0.05 & 0.05 & 0.06 & 0.05 & 0.06 & 0.05 & 0.07 \\
\hline $\mathrm{Re}_{2} \mathrm{O}_{7}$ & 0.10 & 0.03 & 0.10 & 0.03 & 0.10 & 0.05 & 0.10 & 0.07 & 0.10 & 0.02 & 0.10 & 0.03 & 0.10 & 0.05 & 0.10 & 0.05 \\
\hline $\mathrm{SiO}_{2}$ & 47.00 & 48.78 & 47.00 & 49.80 & 47.70 & 48.55 & 47.70 & 48.87 & 47.70 & 49.91 & 47.70 & 50.31 & 47.95 & 49.36 & 47.95 & 49.14 \\
\hline $\mathrm{SO}_{3}$ & 4.50 & 0.98 & 4.50 & 0.93 & 0.78 & 0.58 & 0.00 & 0.01 & 4.50 & 0.98 & 4.50 & 0.95 & 0.78 & 0.58 & 0.00 & BDL \\
\hline $\mathrm{TiO}_{2}$ & 0.00 & 0.02 & 0.00 & 0.01 & 0.00 & 0.01 & 0.00 & 0.02 & 0.00 & 0.01 & 0.00 & 0.01 & 0.00 & 0.02 & 0.00 & 0.02 \\
\hline $\mathrm{ZnO}$ & 4.00 & 3.90 & 4.00 & 3.82 & 4.00 & 4.15 & 4.00 & 3.85 & 4.00 & 3.65 & 4.00 & 3.69 & 4.00 & 4.00 & 4.00 & 3.86 \\
\hline $\mathrm{ZrO}_{2}$ & 3.15 & 3.38 & 3.15 & 3.47 & 3.15 & 2.70 & 3.15 & 3.53 & 3.15 & 3.23 & 3.15 & 3.33 & 3.15 & 3.36 & 3.15 & 3.54 \\
\hline Sum* & 103.80 & 101.30 & 103.80 & 101.30 & 100.10 & 101.20 & 99.30 & 101.20 & 103.80 & 101.30 & 103.80 & 101.30 & 100.10 & 101.10 & 99.30 & 101.20 \\
\hline
\end{tabular}


Table 4.7. XRF Analysis of Glasses Tested for LAW AZ-101 (cont'd).

\begin{tabular}{|c|c|c|c|c|c|c|c|c|c|c|c|c|c|c|c|c|}
\hline \multirow{2}{*}{$\begin{array}{c}\text { Oxide } \\
-\end{array}$} & \multicolumn{2}{|c|}{ LAWB80S4 } & \multicolumn{2}{|c|}{ LAWB80S4W } & \multicolumn{2}{|c|}{ LAWB81 } & \multicolumn{2}{|c|}{ LAWB81S4 } & \multicolumn{2}{|c|}{ LAWB81S4W } & \multicolumn{2}{|c|}{ LAWB82 } & \multicolumn{2}{|c|}{ LAWB82S4 } & \multicolumn{2}{|c|}{ LAWB82S4W } \\
\hline & Target & $\mathrm{XRF}$ & Target & XRF & Target & XRF & Target & XRF & Target & $\mathrm{XRF}$ & Target & $\mathrm{XRF}$ & Target & $\mathrm{XRF}$ & Target & $\mathrm{XRF}$ \\
\hline $\mathrm{Al}_{2} \mathrm{O}_{3}$ & 6.15 & 6.48 & 6.15 & 6.43 & 6.15 & 5.69 & 6.15 & 5.84 & 6.15 & 5.90 & 6.15 & 5.61 & 6.15 & 5.73 & 6.15 & 5.68 \\
\hline $\mathrm{B}_{2} \mathrm{O}_{3}$ & 12.33 & N/A & 12.33 & N/A & 12.33 & N/A & 12.33 & N/A & 12.33 & N/A & 10.08 & N/A & 10.08 & N/A & 10.08 & N/A \\
\hline $\mathrm{CaO}$ & 7.12 & 6.63 & 7.12 & 6.60 & 7.12 & 7.24 & 7.12 & 6.94 & 7.12 & 6.74 & 7.12 & 7.15 & 7.12 & 6.93 & 7.12 & 6.65 \\
\hline $\mathrm{Cl}$ & 0.01 & 0.01 & 0.01 & 0.00 & 0.01 & 0.01 & 0.01 & $\mathrm{BDL}$ & 0.01 & $\mathrm{BDL}$ & 0.01 & $\mathrm{BDL}$ & 0.01 & $\mathrm{BDL}$ & 0.01 & $\mathrm{BDL}$ \\
\hline $\mathrm{Cr}_{2} \mathrm{O}_{3}$ & 0.05 & 0.04 & 0.05 & 0.04 & 0.05 & 0.32 & 0.05 & 0.04 & 0.05 & 0.04 & 0.05 & 0.26 & 0.05 & 0.04 & 0.05 & 0.04 \\
\hline $\mathrm{F}$ & 0.08 & N/A & 0.08 & N/A & 0.08 & N/A & 0.08 & N/A & 0.08 & N/A & 0.08 & N/A & 0.08 & N/A & 0.08 & N/A \\
\hline $\mathrm{Fe}_{2} \mathrm{O}_{3}$ & 3.25 & 3.18 & 3.25 & 3.26 & 3.25 & 4.14 & 3.25 & 3.49 & 3.25 & 3.44 & 9.50 & 10.45 & 9.50 & 9.93 & 9.50 & 9.84 \\
\hline $\mathrm{K}_{2} \mathrm{O}$ & 1.99 & 1.58 & 1.99 & 1.58 & 0.23 & 0.29 & 0.23 & 0.26 & 0.23 & 0.25 & 0.23 & 0.29 & 0.23 & 0.26 & 0.23 & 0.25 \\
\hline $\mathrm{Li}_{2} \mathrm{O}$ & 3.51 & N/A & 3.51 & N/A & 4.26 & N/A & 4.26 & N/A & 4.26 & N/A & 4.26 & N/A & 4.26 & N/A & 4.26 & N/A \\
\hline $\mathrm{MgO}$ & 2.97 & 3.02 & 2.97 & 2.85 & 2.97 & 2.96 & 2.97 & 3.11 & 2.97 & 2.86 & 1.48 & 1.37 & 1.48 & 1.52 & 1.48 & 1.54 \\
\hline $\mathrm{Na}_{2} \mathrm{O}$ & 6.62 & 6.14 & 6.62 & 5.97 & 6.62 & 6.72 & 6.62 & 6.57 & 6.62 & 6.18 & 6.62 & 6.57 & 6.62 & 6.26 & 6.62 & 6.17 \\
\hline $\mathrm{P}_{2} \mathrm{O}_{5}$ & 0.05 & 0.06 & 0.05 & 0.08 & 0.05 & 0.05 & 0.05 & 0.06 & 0.05 & 0.06 & 0.05 & 0.06 & 0.05 & 0.06 & 0.05 & 0.07 \\
\hline $\mathrm{Re}_{2} \mathrm{O}_{7}$ & 0.10 & 0.02 & 0.10 & 0.02 & 0.10 & 0.06 & 0.10 & 0.04 & 0.10 & 0.03 & 0.10 & 0.05 & 0.10 & 0.02 & 0.10 & 0.02 \\
\hline $\mathrm{SiO}_{2}$ & 47.95 & 50.29 & 47.95 & 50.58 & 47.95 & 47.67 & 47.95 & 48.50 & 47.95 & 49.46 & 45.44 & 45.51 & 45.44 & 45.72 & 45.44 & 46.81 \\
\hline $\mathrm{SO}_{3}$ & 4.50 & 1.05 & 4.50 & 1.04 & 0.78 & 0.60 & 4.50 & 1.31 & 4.50 & 1.14 & 0.78 & 0.48 & 4.50 & 1.59 & 4.50 & 1.07 \\
\hline $\mathrm{TiO}_{2}$ & 0.00 & 0.01 & 0.00 & 0.01 & 0.00 & 0.01 & 0.00 & 0.01 & 0.00 & 0.01 & 0.00 & 0.01 & 0.00 & 0.00 & 0.00 & 0.01 \\
\hline $\mathrm{ZnO}$ & 4.00 & 3.64 & 4.00 & 3.66 & 5.00 & 5.07 & 5.00 & 5.05 & 5.00 & 4.91 & 5.00 & 5.01 & 5.00 & 5.06 & 5.00 & 4.94 \\
\hline $\mathrm{ZrO}_{2}$ & 3.15 & 3.25 & 3.15 & 3.26 & 3.15 & 3.81 & 3.15 & 3.64 & 3.15 & 3.75 & 3.15 & 3.73 & 3.15 & 3.71 & 3.15 & 3.66 \\
\hline Sum* & 103.80 & 101.20 & 103.80 & 101.20 & 100.10 & 101.20 & 103.80 & 101.40 & 103.80 & 101.40 & 100.10 & 100.90 & 103.80 & 101.20 & 103.80 & 101.10 \\
\hline
\end{tabular}


Table 4.7. XRF Analysis of Glasses Tested for LAW AZ-101.

\begin{tabular}{|c|c|c|c|c|c|c|c|c|c|c|c|c|c|c|c|c|}
\hline \multirow{2}{*}{$\begin{array}{c}\text { Oxide } \\
-\end{array}$} & \multicolumn{2}{|c|}{ LAWB83 } & \multicolumn{2}{|c|}{ LAWB83S0 } & \multicolumn{2}{|c|}{ LAWB83S4 } & \multicolumn{2}{|c|}{ LAWB83S4W } & \multicolumn{2}{|c|}{ LAWB83S4-2 } & \multicolumn{2}{|c|}{ LAWB83S4W-2 } & \multicolumn{2}{|c|}{ LAWB84 } & \multicolumn{2}{|c|}{ LAWB84S4 } \\
\hline & Target & XRF & Target & XRF & Target & XRF & Target & XRF & Target & XRF & Target & XRF & Target & XRF & Target & XRF \\
\hline $\mathrm{Al}_{2} \mathrm{O}_{3}$ & 6.18 & 5.82 & 6.18 & 6.16 & 6.18 & 6.29 & 6.18 & 5.68 & 6.18 & 5.96 & 6.18 & 5.91 & 6.18 & 5.96 & 6.18 & 6.16 \\
\hline $\mathrm{B}_{2} \mathrm{O}_{3}$ & 10.03 & N/A & 10.03 & N/A & 10.03 & N/A & 10.03 & N/A & 10.03 & N/A & 10.03 & N/A & 10.03 & N/A & 10.03 & N/A \\
\hline $\mathrm{CaO}$ & 6.78 & 6.95 & 6.78 & 6.80 & 6.78 & 6.36 & 6.78 & 6.77 & 6.78 & 6.51 & 6.78 & 6.39 & 6.68 & 6.57 & 6.68 & 6.32 \\
\hline $\mathrm{Cl}$ & 0.01 & $\mathrm{BDL}$ & 0.01 & BDL & 0.01 & $\mathrm{BDL}$ & 0.01 & $\mathrm{BDL}$ & 0.01 & $\mathrm{BDL}$ & 0.01 & $\mathrm{BDL}$ & 0.01 & $\mathrm{BDL}$ & 0.01 & BDL \\
\hline $\mathrm{Cr}_{2} \mathrm{O}_{3}$ & 0.04 & 0.04 & 0.04 & 0.04 & 0.04 & 0.03 & 0.04 & 0.04 & 0.04 & 0.03 & 0.04 & 0.04 & 0.04 & 0.04 & 0.04 & 0.04 \\
\hline $\mathrm{F}$ & 0.06 & N/A & 0.06 & N/A & 0.06 & N/A & 0.06 & N/A & 0.06 & N/A & 0.06 & N/A & 0.06 & N/A & 0.06 & N/A \\
\hline $\mathrm{Fe}_{2} \mathrm{O}_{3}$ & 5.29 & 5.70 & 5.29 & 5.52 & 5.29 & 5.43 & 5.29 & 6.16 & 5.29 & 5.65 & 5.29 & 5.57 & 5.29 & 5.41 & 5.29 & 5.39 \\
\hline $\mathrm{K}_{2} \mathrm{O}$ & 0.19 & 0.27 & 0.19 & 0.27 & 0.19 & 0.26 & 0.19 & 0.26 & 0.19 & 0.25 & 0.19 & 0.24 & 0.19 & 0.25 & 0.19 & 0.25 \\
\hline $\mathrm{Li}_{2} \mathrm{O}$ & 4.31 & N/A & 4.31 & N/A & 4.31 & N/A & 4.31 & N/A & 4.31 & N/A & 4.31 & N/A & 4.40 & N/A & 4.40 & N/A \\
\hline $\mathrm{MgO}$ & 2.97 & 2.90 & 2.97 & 2.96 & 2.97 & 2.90 & 2.97 & 2.83 & 2.97 & 3.04 & 2.97 & 2.92 & 2.97 & 3.01 & 2.97 & 3.00 \\
\hline $\mathrm{Na}_{2} \mathrm{O}$ & 5.47 & 5.76 & 5.47 & 5.80 & 5.47 & 5.27 & 5.47 & 4.92 & 5.47 & 5.55 & 5.47 & 5.00 & 5.47 & 6.18 & 5.47 & 5.43 \\
\hline $\mathrm{P}_{2} \mathrm{O}_{5}$ & 0.04 & 0.05 & 0.04 & 0.05 & 0.04 & 0.07 & 0.04 & 0.05 & 0.04 & 0.05 & 0.04 & 0.05 & 0.04 & 0.06 & 0.04 & 0.05 \\
\hline $\mathrm{Re}_{2} \mathrm{O}_{7}$ & 0.10 & 0.06 & 0.10 & 0.06 & 0.10 & 0.02 & 0.10 & 0.03 & 0.10 & 0.02 & 0.10 & 0.02 & 0.10 & 0.05 & 0.10 & 0.02 \\
\hline $\mathrm{SiO}_{2}$ & 48.60 & 48.37 & 48.60 & 49.05 & 48.60 & 49.35 & 48.60 & 47.35 & 48.60 & 48.16 & 48.60 & 49.21 & 48.60 & 49.06 & 48.60 & 49.26 \\
\hline $\mathrm{SO}_{3}$ & 0.65 & 0.49 & 0.00 & 0.00 & 4.50 & 1.20 & 4.50 & 1.02 & 4.50 & 1.16 & 4.50 & 1.06 & 0.65 & 0.44 & 4.50 & 1.31 \\
\hline $\mathrm{TiO}_{2}$ & 1.39 & 1.57 & 1.39 & 1.52 & 1.39 & 1.51 & 1.39 & 1.70 & 1.39 & 1.60 & 1.39 & 1.59 & 1.39 & 1.48 & 1.39 & 1.47 \\
\hline $\mathrm{ZnO}$ & 4.84 & 4.96 & 4.84 & 4.79 & 4.84 & 4.64 & 4.84 & 5.66 & 4.84 & 5.06 & 4.84 & 4.96 & 4.84 & 4.67 & 4.84 & 4.61 \\
\hline $\mathrm{ZrO}_{2}$ & 3.16 & 3.85 & 3.16 & 3.72 & 3.16 & 3.54 & 3.16 & 4.45 & 3.16 & 3.91 & 3.16 & 3.93 & 3.16 & 3.53 & 3.16 & 3.51 \\
\hline Sum* & 100.10 & 101.10 & 99.50 & 101.10 & 104.00 & 101.20 & 104.00 & 101.20 & 104.00 & 101.30 & 104.00 & 101.20 & 100.10 & 101.10 & 104.00 & 101.20 \\
\hline
\end{tabular}


Table 4.7. XRF Analysis of Glasses Tested for LAW AZ-101 (cont'd).

\begin{tabular}{|c|c|c|c|c|c|c|c|c|c|c|c|c|c|c|c|c|}
\hline \multirow{2}{*}{$\begin{array}{c}\text { Oxide } \\
- \\
\end{array}$} & \multicolumn{2}{|c|}{ LAWB84S4W } & \multicolumn{2}{|c|}{ LAWB85 } & \multicolumn{2}{|c|}{ LAWB85S4 } & \multicolumn{2}{|c|}{ LAWB85S4W } & \multicolumn{2}{|c|}{ LAWB86 } & \multicolumn{2}{|c|}{ LAWB86S4 } & \multicolumn{2}{|c|}{ LAWB86S4W } & \multicolumn{2}{|c|}{ LAWB89 } \\
\hline & Target & XRF & Target & XRF & Target & XRF & Target & XRF & Target & XRF & Target & XRF & Target & XRF & Target & XRF \\
\hline $\mathrm{Al}_{2} \mathrm{O}_{3}$ & 6.18 & 5.93 & 6.18 & 5.98 & 6.18 & 6.32 & 6.18 & 5.96 & 6.18 & 6.37 & 6.18 & 6.28 & 6.18 & 5.83 & 6.18 & 6.18 \\
\hline $\mathrm{B}_{2} \mathrm{O}_{3}$ & 10.03 & N/A & 11.52 & N/A & 11.52 & N/A & 11.52 & N/A & 12.41 & N/A & 12.41 & N/A & 12.41 & N/A & 10.03 & N/A \\
\hline $\mathrm{CaO}$ & 6.68 & 6.35 & 5.28 & 5.39 & 5.28 & 4.94 & 5.28 & 4.99 & 5.73 & 5.61 & 5.73 & 5.48 & 5.73 & 5.71 & 6.78 & 6.72 \\
\hline $\mathrm{Cl}$ & 0.01 & $\mathrm{BDL}$ & 0.01 & 0.00 & 0.01 & BDL & 0.01 & BDL & 0.01 & 0.01 & 0.01 & 0.01 & 0.01 & 0.01 & 0.01 & 0.01 \\
\hline $\mathrm{Cr}_{2} \mathrm{O}_{3}$ & 0.04 & 0.04 & 0.04 & 0.04 & 0.04 & 0.03 & 0.04 & 0.04 & 0.04 & 0.04 & 0.04 & 0.04 & 0.04 & 0.04 & 0.04 & 0.04 \\
\hline $\mathrm{F}$ & 0.06 & N/A & 0.06 & N/A & 0.06 & N/A & 0.06 & N/A & 0.06 & N/A & 0.06 & N/A & 0.06 & N/A & 0.06 & N/A \\
\hline $\mathrm{Fe}_{2} \mathrm{O}_{3}$ & 5.29 & 5.54 & 5.29 & 5.62 & 5.29 & 5.41 & 5.29 & 5.59 & 5.29 & 5.26 & 5.29 & 5.42 & 5.29 & 5.99 & 5.29 & 5.46 \\
\hline $\mathrm{K}_{2} \mathrm{O}$ & 0.19 & 0.24 & 0.19 & 0.26 & 0.19 & 0.27 & 0.19 & 0.25 & 0.19 & 0.29 & 0.19 & 0.26 & 0.19 & 0.25 & 0.19 & 0.29 \\
\hline $\mathrm{Li}_{2} \mathrm{O}$ & 4.40 & N/A & 4.31 & N/A & 4.31 & N/A & 4.31 & N/A & 4.35 & N/A & 4.35 & N/A & 4.35 & N/A & 5.00 & N/A \\
\hline MgO & 2.97 & 2.90 & 2.97 & 2.93 & 2.97 & 2.87 & 2.97 & 2.82 & 2.97 & 2.92 & 2.97 & 2.98 & 2.97 & 2.86 & 2.97 & 2.94 \\
\hline $\mathrm{Na}_{2} \mathrm{O}$ & 5.47 & 5.33 & 5.47 & 5.50 & 5.47 & 5.08 & 5.47 & 5.44 & 5.47 & 6.28 & 5.47 & 4.98 & 5.47 & 4.89 & 4.08 & 5.08 \\
\hline $\mathrm{P}_{2} \mathrm{O}_{5}$ & 0.04 & 0.04 & 0.04 & 0.10 & 0.04 & 0.05 & 0.04 & 0.04 & 0.04 & 0.07 & 0.04 & 0.06 & 0.04 & 0.05 & 0.04 & 0.05 \\
\hline $\mathrm{Re}_{2} \mathrm{O}_{7}$ & 0.10 & 0.02 & 0.10 & 0.06 & 0.10 & 0.03 & 0.10 & 0.02 & 0.10 & 0.06 & 0.10 & 0.03 & 0.10 & 0.04 & 0.10 & 0.06 \\
\hline $\mathrm{SiO}_{2}$ & 48.60 & 48.79 & 48.60 & 48.84 & 48.60 & 49.55 & 48.60 & 48.74 & 48.60 & 49.24 & 48.60 & 49.56 & 48.60 & 48.22 & 49.30 & 48.61 \\
\hline $\mathrm{SO}_{3}$ & 4.50 & 1.10 & 0.65 & 0.49 & 4.50 & 1.16 & 4.50 & 1.01 & 0.65 & 0.43 & 4.50 & 1.26 & 4.50 & 1.02 & 0.65 & 0.44 \\
\hline $\mathrm{TiO}_{2}$ & 1.39 & 1.58 & 1.39 & 1.55 & 1.39 & 1.50 & 1.39 & 1.57 & 0.00 & 0.03 & 0.00 & 0.01 & 0.00 & 0.01 & 1.39 & 1.55 \\
\hline $\mathrm{ZnO}$ & 4.84 & 4.98 & 4.84 & 4.87 & 4.84 & 4.62 & 4.84 & 5.01 & 4.84 & 4.50 & 4.84 & 4.66 & 4.84 & 5.41 & 4.84 & 4.92 \\
\hline $\mathrm{ZrO}_{2}$ & 3.16 & 3.93 & 3.16 & 3.75 & 3.16 & 3.61 & 3.16 & 3.96 & 3.16 & 3.40 & 3.16 & 3.60 & 3.16 & 4.30 & 3.16 & 3.86 \\
\hline Sum* & 104.00 & 101.20 & 100.10 & 101.20 & 104.00 & 101.30 & 104.00 & 101.30 & 100.10 & 101.30 & 103.90 & 101.40 & 103.90 & 101.40 & 100.10 & 101.20 \\
\hline
\end{tabular}


Table 4.7. XRF Analysis of Glasses Tested for Tank AZ-101 (cont'd).

\begin{tabular}{|c|c|c|c|c|c|c|c|c|c|c|c|c|c|c|c|c|}
\hline \multirow{2}{*}{$\begin{array}{c}\text { Oxide } \\
-\end{array}$} & \multicolumn{2}{|c|}{ LAWB89R1 } & \multicolumn{2}{|c|}{ LAWB89R2 } & \multicolumn{2}{|c|}{ LAWB89R3 } & \multicolumn{2}{|c|}{ LAWB89R4 } & \multicolumn{2}{|c|}{ LAWB89S4 } & \multicolumn{2}{|c|}{ LAWB89S4W } & \multicolumn{2}{|c|}{ LAWB90 } & \multicolumn{2}{|c|}{ LAWB90R1 } \\
\hline & Target & XRF & Target & XRF & Target & XRF & Target & XRF & Target & XRF & Target & $\mathrm{XRF}$ & Target & XRF & Target & XRF \\
\hline $\mathrm{Al}_{2} \mathrm{O}_{3}$ & 6.18 & 6.20 & 6.18 & 6.06 & 6.18 & 5.99 & 6.18 & 5.89 & 6.18 & 5.96 & 6.18 & 5.72 & 6.18 & 5.76 & 6.18 & 6.30 \\
\hline $\mathrm{B}_{2} \mathrm{O}_{3}$ & 10.03 & N/A & 10.03 & N/A & 10.03 & N/A & 10.03 & N/A & 10.03 & N/A & 10.03 & N/A & 10.03 & N/A & 10.03 & N/A \\
\hline $\mathrm{CaO}$ & 6.78 & 6.70 & 6.78 & 6.60 & 6.78 & 6.71 & 6.78 & 6.84 & 6.78 & 6.39 & 6.78 & 6.43 & 6.78 & 6.74 & 6.78 & 6.66 \\
\hline $\mathrm{Cl}$ & 0.01 & 0.01 & 0.01 & $\mathrm{BDL}$ & 0.01 & $\mathrm{BDL}$ & 0.01 & $\mathrm{BDL}$ & 0.01 & $\mathrm{BDL}$ & 0.01 & $\mathrm{BDL}$ & 0.01 & 0.00 & 0.01 & $\mathrm{BDL}$ \\
\hline $\mathrm{Cr}_{2} \mathrm{O}_{3}$ & 0.04 & 0.03 & 0.04 & 0.03 & 0.04 & 0.04 & 0.04 & 0.04 & 0.04 & 0.03 & 0.04 & 0.04 & 0.04 & 0.19 & 0.04 & 0.03 \\
\hline $\mathrm{F}$ & 0.06 & N/A & 0.06 & N/A & 0.06 & N/A & 0.06 & N/A & 0.06 & N/A & 0.06 & 0.00 & 0.06 & N/A & 0.06 & N/A \\
\hline $\mathrm{Fe}_{2} \mathrm{O}_{3}$ & 5.29 & 5.47 & 5.29 & 5.30 & 5.29 & 5.36 & 5.29 & 5.57 & 5.29 & 5.38 & 5.29 & 5.55 & 5.29 & 5.93 & 5.29 & 5.32 \\
\hline $\mathrm{K}_{2} \mathrm{O}$ & 0.19 & 0.26 & 0.19 & 0.28 & 0.19 & 0.26 & 0.19 & 0.26 & 0.19 & 0.24 & 0.19 & 0.23 & 0.19 & 0.25 & 0.19 & 0.25 \\
\hline $\mathrm{Li}_{2} \mathrm{O}$ & 5.00 & N/A & 5.00 & N/A & 5.00 & N/A & 5.00 & N/A & 5.00 & N/A & 5.00 & N/A & 3.61 & N/A & 3.61 & N/A \\
\hline $\mathrm{MgO}$ & 2.97 & 2.96 & 2.97 & 2.99 & 2.97 & 2.93 & 2.97 & 2.74 & 2.97 & 2.92 & 2.97 & 2.81 & 2.97 & 3.04 & 2.97 & 3.02 \\
\hline $\mathrm{Na}_{2} \mathrm{O}$ & 4.08 & 4.66 & 4.08 & 4.86 & 4.08 & 4.59 & 4.08 & 4.34 & 4.08 & 4.23 & 4.08 & 3.90 & 6.87 & 7.48 & 6.87 & 7.45 \\
\hline $\mathrm{P}_{2} \mathrm{O}_{5}$ & 0.04 & 0.05 & 0.04 & 0.04 & 0.04 & 0.04 & 0.04 & 0.06 & 0.04 & 0.05 & 0.04 & 0.05 & 0.04 & 0.05 & 0.04 & 0.05 \\
\hline $\mathrm{Re}_{2} \mathrm{O}_{7}$ & 0.10 & 0.05 & 0.10 & 0.04 & 0.10 & 0.04 & 0.10 & 0.05 & 0.10 & 0.02 & 0.10 & 0.02 & 0.10 & 0.04 & 0.10 & 0.04 \\
\hline $\mathrm{SiO}_{2}$ & 49.30 & 49.62 & 49.30 & 49.64 & 49.30 & 49.56 & 49.30 & 49.59 & 49.30 & 49.86 & 49.30 & 49.95 & 47.90 & 47.21 & 47.90 & 48.68 \\
\hline $\mathrm{SO}_{3}$ & 0.65 & 0.52 & 0.65 & 0.48 & 0.65 & 0.55 & 0.65 & 0.54 & 4.50 & 1.33 & 4.50 & 1.28 & 0.65 & 0.34 & 0.65 & 0.49 \\
\hline $\mathrm{TiO}_{2}$ & 1.39 & 1.41 & 1.39 & 1.49 & 1.39 & 1.51 & 1.39 & 1.53 & 1.39 & 1.50 & 1.39 & 1.53 & 1.39 & 1.56 & 1.39 & 1.41 \\
\hline $\mathrm{ZnO}$ & 4.84 & 4.68 & 4.84 & 4.76 & 4.84 & 4.68 & 4.84 & 4.84 & 4.84 & 4.65 & 4.84 & 4.81 & 4.84 & 5.03 & 4.84 & 4.63 \\
\hline $\mathrm{ZrO}_{2}$ & 3.16 & 3.50 & 3.16 & 3.54 & 3.16 & 3.65 & 3.16 & 3.79 & 3.16 & 3.64 & 3.16 & 3.85 & 3.16 & 3.90 & 3.16 & 3.19 \\
\hline Sum* & 100.10 & 101.20 & 100.10 & 101.10 & 100.10 & 101.20 & 100.10 & 101.10 & 104.00 & 101.20 & 104.00 & 101.20 & 100.10 & 101.10 & 100.10 & 101.20 \\
\hline
\end{tabular}


Table 4.7. XRF Analysis of Glasses Tested for LAW AZ-101 (cont'd).

\begin{tabular}{|c|c|c|c|c|c|c|c|c|c|c|c|c|c|c|c|c|}
\hline \multirow{2}{*}{$\begin{array}{c}\text { Oxide } \\
-\end{array}$} & \multicolumn{2}{|c|}{ LAWB91 } & \multicolumn{2}{|c|}{ LAWB91R1 } & \multicolumn{2}{|c|}{ LAWB91S4 } & \multicolumn{2}{|c|}{ LAWB91S4W } & \multicolumn{2}{|c|}{ LAWB92 } & \multicolumn{2}{|c|}{ LAWB92S4 } & \multicolumn{2}{|c|}{ LAWB92S4W } & \multicolumn{2}{|c|}{ LAWB92R1 } \\
\hline & Target & XRF & Target & XRF & Target & XRF & Target & XRF & Target & XRF & Target & XRF & Target & XRF & Target & XRF \\
\hline $\mathrm{Al}_{2} \mathrm{O}_{3}$ & 6.18 & 5.83 & 6.18 & 5.98 & 6.18 & 5.94 & 6.18 & 5.87 & 6.18 & 5.70 & 6.18 & 5.99 & 6.18 & 5.79 & 6.18 & 6.22 \\
\hline $\mathrm{B}_{2} \mathrm{O}_{3}$ & 10.03 & N/A & 10.03 & N/A & 10.03 & N/A & 10.03 & N/A & 10.03 & N/A & 10.03 & N/A & 10.03 & N/A & 10.03 & N/A \\
\hline $\mathrm{CaO}$ & 6.78 & 6.66 & 6.78 & 6.80 & 6.78 & 6.35 & 6.78 & 6.19 & 6.78 & 6.71 & 6.78 & 6.46 & 6.78 & 6.36 & 6.78 & 6.66 \\
\hline $\mathrm{Cl}$ & 0.01 & $\mathrm{BDL}$ & 0.01 & BDL & 0.01 & BDL & 0.01 & BDL & 0.01 & $\mathrm{BDL}$ & 0.01 & $\mathrm{BDL}$ & 0.01 & $\mathrm{BDL}$ & 0.01 & BDL \\
\hline $\mathrm{Cr}_{2} \mathrm{O}_{3}$ & 0.04 & 0.23 & 0.04 & 0.04 & 0.04 & 0.04 & 0.04 & 0.04 & 0.04 & 0.21 & 0.04 & 0.03 & 0.04 & 0.03 & 0.04 & 0.04 \\
\hline $\mathrm{F}$ & 0.06 & N/A & 0.06 & N/A & 0.06 & N/A & 0.06 & N/A & 0.06 & N/A & 0.06 & N/A & 0.06 & N/A & 0.06 & N/A \\
\hline $\mathrm{Fe}_{2} \mathrm{O}_{3}$ & 5.29 & 5.88 & 5.29 & 5.56 & 5.29 & 5.41 & 5.29 & 5.45 & 5.29 & 5.94 & 5.29 & 5.52 & 5.29 & 5.54 & 5.29 & 5.27 \\
\hline $\mathrm{K}_{2} \mathrm{O}$ & 0.19 & 0.24 & 0.19 & 0.26 & 0.19 & 0.22 & 0.19 & 0.23 & 0.19 & 0.24 & 0.19 & 0.23 & 0.19 & 0.23 & 0.19 & 0.25 \\
\hline $\mathrm{Li}_{2} \mathrm{O}$ & 2.92 & N/A & 2.92 & N/A & 2.92 & N/A & 2.92 & N/A & 2.22 & N/A & 2.22 & N/A & 2.22 & N/A & 2.22 & N/A \\
\hline MgO & 2.97 & 3.09 & 2.97 & 2.95 & 2.97 & 2.99 & 2.97 & 2.85 & 2.97 & 3.00 & 2.97 & 2.92 & 2.97 & 2.82 & 2.97 & 3.08 \\
\hline $\mathrm{Na}_{2} \mathrm{O}$ & 8.72 & 9.17 & 8.72 & 9.21 & 8.72 & 8.40 & 8.72 & 8.10 & 10.11 & 10.93 & 10.11 & 9.11 & 10.11 & 8.89 & 10.11 & 10.80 \\
\hline $\mathrm{P}_{2} \mathrm{O}_{5}$ & 0.04 & 0.05 & 0.04 & 0.05 & 0.04 & 0.05 & 0.04 & 0.05 & 0.04 & $\mathrm{BDL}$ & 0.04 & 0.05 & 0.04 & 0.06 & 0.04 & 0.06 \\
\hline $\mathrm{Re}_{2} \mathrm{O}_{7}$ & 0.10 & 0.05 & 0.10 & 0.03 & 0.10 & 0.03 & 0.10 & 0.04 & 0.10 & 0.04 & 0.10 & 0.04 & 0.10 & 0.04 & 0.10 & 0.05 \\
\hline $\mathrm{SiO}_{2}$ & 46.74 & 46.39 & 46.74 & 46.90 & 46.74 & 48.21 & 46.74 & 48.81 & 46.05 & 45.38 & 46.05 & 47.81 & 46.05 & 48.45 & 46.05 & 46.39 \\
\hline $\mathrm{SO}_{3}$ & 0.65 & 0.37 & 0.65 & 0.36 & 4.50 & 0.81 & 4.50 & 0.71 & 0.65 & 0.43 & 4.50 & 0.77 & 0.65 & 0.64 & 4.50 & 0.39 \\
\hline $\mathrm{TiO}_{2}$ & 1.39 & 1.54 & 1.39 & 1.45 & 1.39 & 1.50 & 1.39 & 1.49 & 1.39 & 1.55 & 1.39 & 1.53 & 1.39 & 1.54 & 1.39 & 1.44 \\
\hline $\mathrm{ZnO}$ & 4.84 & 4.90 & 4.84 & 4.83 & 4.84 & 4.61 & 4.84 & 4.62 & 4.84 & 4.91 & 4.84 & 4.73 & 4.84 & 4.70 & 4.84 & 4.65 \\
\hline $\mathrm{ZrO}_{2}$ & 3.16 & 3.81 & 3.16 & 3.80 & 3.16 & 3.61 & 3.16 & 3.71 & 3.16 & 3.82 & 3.16 & 3.73 & 3.16 & 3.78 & 3.16 & 3.66 \\
\hline Sum* & 100.10 & 101.10 & 100.10 & 101.10 & 104.00 & 101.10 & 104.00 & 101.08 & 100.10 & 101.11 & 104.00 & 101.15 & 100.10 & 101.09 & 104.00 & 101.20 \\
\hline
\end{tabular}


Table 4.7. XRF Analysis of Glasses Tested for LAW AZ-101 (cont'd).

\begin{tabular}{|c|c|c|c|c|c|c|c|c|c|c|c|c|c|c|c|c|c|c|}
\hline \multirow{2}{*}{$\begin{array}{c}\text { Oxide } \\
-\end{array}$} & \multicolumn{2}{|c|}{ LAWB93 } & \multicolumn{2}{|c|}{ LAWB93S4 } & \multicolumn{2}{|c|}{ LAWB93S4W } & \multicolumn{2}{|c|}{ LAWB94 } & \multicolumn{2}{|c|}{ LAWB94S4 } & \multicolumn{2}{|c|}{ LAWB94S4W } & \multicolumn{2}{|c|}{ LAWB95 } & \multicolumn{2}{|c|}{ LAWB95S4 } & \multicolumn{2}{|c|}{ LAWB95S4W } \\
\hline & Target & XRF & Target & XRF & Target & XRF & Target & XRF & Target & XRF & Target & XRF & Target & XRF & Target & XRF & Target & XRF \\
\hline $\mathrm{Al}_{2} \mathrm{O}_{3}$ & 6.18 & 5.63 & 6.18 & 5.83 & 6.18 & 5.72 & 6.18 & 5.78 & 6.18 & 5.88 & 6.18 & 5.69 & 6.18 & 5.94 & 6.18 & 5.77 & 6.18 & 5.61 \\
\hline $\mathrm{B}_{2} \mathrm{O}_{3}$ & 10.03 & N/A & 10.03 & N/A & 10.03 & N/A & 10.03 & N/A & 10.03 & N/A & 10.03 & N/A & 10.03 & N/A & 10.03 & N/A & 10.03 & N/A \\
\hline $\mathrm{CaO}$ & 6.78 & 6.69 & 6.78 & 6.39 & 6.78 & 6.36 & 6.78 & 6.79 & 6.78 & 6.33 & 6.78 & 6.31 & 6.78 & 6.57 & 6.78 & 6.39 & 6.78 & 6.31 \\
\hline $\mathrm{Cl}$ & 0.01 & $\mathrm{BDL}$ & 0.01 & $\mathrm{BDL}$ & 0.01 & $\mathrm{BDL}$ & 0.01 & 0.01 & 0.01 & $\mathrm{BDL}$ & 0.01 & $\mathrm{BDL}$ & 0.01 & 0.00 & 0.01 & BDL & 0.01 & 0.01 \\
\hline $\mathrm{Cr}_{2} \mathrm{O}_{3}$ & 0.04 & 0.29 & 0.04 & 0.04 & 0.04 & 0.04 & 0.04 & 0.20 & 0.04 & 0.04 & 0.04 & 0.04 & 0.04 & 0.22 & 0.04 & 0.04 & 0.04 & 0.04 \\
\hline $\mathrm{F}$ & 0.06 & N/A & 0.06 & N/A & 0.06 & 0.00 & 0.06 & N/A & 0.06 & N/A & 0.06 & N/A & 0.06 & N/A & 0.06 & N/A & 0.06 & N/A \\
\hline $\mathrm{Fe}_{2} \mathrm{O}_{3}$ & 5.29 & 6.18 & 5.29 & 5.49 & 5.29 & 5.52 & 5.29 & 5.81 & 5.29 & 5.30 & 5.29 & 5.41 & 5.29 & 5.76 & 5.29 & 5.39 & 5.29 & 5.41 \\
\hline $\mathrm{K}_{2} \mathrm{O}$ & 0.19 & 0.26 & 0.19 & 0.23 & 0.19 & 0.22 & 0.19 & 0.25 & 0.19 & 0.23 & 0.19 & 0.23 & 0.19 & 0.24 & 0.19 & 0.23 & 0.19 & 0.24 \\
\hline $\mathrm{Li}_{2} \mathrm{O}$ & 4.66 & N/A & 4.66 & N/A & 4.66 & N/A & 5.36 & N/A & 5.36 & N/A & 5.36 & N/A & 5.77 & N/A & 5.77 & N/A & 5.77 & N/A \\
\hline $\mathrm{MgO}$ & 2.97 & 2.88 & 2.97 & 2.90 & 2.97 & 2.90 & 2.97 & 2.96 & 2.97 & 2.95 & 2.97 & 2.88 & 2.97 & 3.05 & 2.97 & 2.78 & 2.97 & 2.73 \\
\hline $\mathrm{Na}_{2} \mathrm{O}$ & 4.78 & 5.23 & 4.78 & 4.73 & 4.78 & 4.80 & 2.46 & 3.79 & 2.46 & 4.10 & 2.46 & 4.00 & 3.38 & 3.30 & 3.38 & 3.59 & 3.38 & 3.98 \\
\hline $\mathrm{P}_{2} \mathrm{O}_{5}$ & 0.04 & 0.05 & 0.04 & 0.05 & 0.04 & 0.05 & 0.04 & 0.04 & 0.04 & 0.05 & 0.04 & 0.05 & 0.04 & 0.06 & 0.04 & 0.05 & 0.04 & 0.04 \\
\hline $\mathrm{Re}_{2} \mathrm{O}_{7}$ & 0.10 & 0.04 & 0.10 & 0.02 & 0.10 & 0.01 & 0.10 & 0.05 & 0.10 & 0.02 & 0.10 & 0.02 & 0.10 & 0.06 & 0.10 & 0.02 & 0.10 & 0.02 \\
\hline $\mathrm{SiO}_{2}$ & 48.94 & 48.12 & 48.94 & 49.70 & 48.94 & 49.76 & 50.17 & 49.64 & 50.17 & 49.97 & 50.17 & 49.94 & 49.64 & 50.15 & 49.64 & 49.95 & 49.64 & 49.81 \\
\hline $\mathrm{SO}_{3}$ & 0.65 & 0.45 & 4.50 & 1.32 & 4.50 & 1.18 & 0.65 & 0.50 & 4.50 & 1.45 & 4.50 & 1.36 & 0.65 & 0.46 & 4.50 & 1.47 & 4.50 & 1.33 \\
\hline $\mathrm{TiO}_{2}$ & 1.39 & 1.50 & 1.39 & 1.45 & 1.39 & 1.47 & 1.39 & 1.52 & 1.39 & 1.47 & 1.39 & 1.49 & 1.39 & 1.47 & 1.39 & 1.47 & 1.39 & 1.51 \\
\hline $\mathrm{ZnO}$ & 4.84 & 5.32 & 4.84 & 4.66 & 4.84 & 4.67 & 4.84 & 4.69 & 4.84 & 4.52 & 4.84 & 4.65 & 4.84 & 4.56 & 4.84 & 4.63 & 4.84 & 4.65 \\
\hline $\mathrm{ZrO}_{2}$ & 3.16 & 3.75 & 3.16 & 3.68 & 3.16 & 3.80 & 3.16 & 3.65 & 3.16 & 3.54 & 3.16 & 3.75 & 3.16 & 3.48 & 3.16 & 3.63 & 3.16 & 3.70 \\
\hline Sum* & 100.10 & 101.10 & 104.00 & 101.20 & 104.00 & 101.20 & 100.10 & 101.06 & 104.00 & 101.22 & 104.00 & 101.19 & 100.10 & 101.09 & 104.00 & 101.20 & 104.00 & 101.17 \\
\hline
\end{tabular}


Table 4.8. XRF Analysis of Glasses Tested for LAW AZ-102.

\begin{tabular}{|c|c|c|c|c|c|c|c|c|c|c|c|c|c|c|c|c|}
\hline \multirow{2}{*}{$\begin{array}{c}\text { Oxide } \\
-\end{array}$} & \multicolumn{2}{|c|}{ LAWB61 } & \multicolumn{2}{|c|}{ LAWB61S4 } & \multicolumn{2}{|c|}{ LAWB61S4W } & \multicolumn{2}{|c|}{ LAWB62 } & \multicolumn{2}{|c|}{ LAWB62S4 } & \multicolumn{2}{|c|}{ LAWB62S4W } & \multicolumn{2}{|c|}{ LAWB63 } & \multicolumn{2}{|c|}{ LAWB63S0 } \\
\hline & Target & XRF & Target & XRF & Target & XRF & Target & XRF & Target & XRF & Target & $\mathrm{XRF}$ & Target & $\mathrm{XRF}$ & Target & $\mathrm{XRF}$ \\
\hline $\mathrm{Al}_{2} \mathrm{O}_{3}$ & 6.17 & 6.29 & 6.17 & 6.36 & 6.17 & 6.03 & 6.17 & 6.06 & 6.17 & 6.16 & 6.17 & 6.08 & 6.55 & 6.53 & 6.55 & 6.92 \\
\hline $\mathrm{B}_{2} \mathrm{O}_{3}$ & 9.91 & N/A & 9.91 & N/A & 9.91 & N/A & 9.91 & N/A & 9.91 & N/A & 9.91 & N/A & 9.91 & N/A & 9.91 & N/A \\
\hline $\mathrm{CaO}$ & 6.67 & 6.52 & 6.67 & 6.19 & 6.67 & 6.34 & 11.95 & 11.78 & 11.95 & 11.30 & 11.95 & 11.56 & 9.31 & 9.28 & 9.31 & 9.11 \\
\hline $\mathrm{Cl}$ & 0.00 & BDL & 0.00 & $\mathrm{BDL}$ & 0.00 & $\mathrm{BDL}$ & 0.00 & $\mathrm{BDL}$ & 0.00 & BDL & 0.00 & 0.01 & 0.00 & 0.01 & 0.00 & 0.01 \\
\hline $\mathrm{Cr}_{2} \mathrm{O}_{3}$ & 0.10 & 0.09 & 0.10 & 0.08 & 0.10 & 0.08 & 0.10 & 0.09 & 0.10 & 0.09 & 0.10 & 0.10 & 0.10 & 0.10 & 0.10 & 0.10 \\
\hline $\mathrm{F}$ & 0.07 & N/A & 0.07 & N/A & 0.07 & N/A & 0.07 & N/A & 0.07 & N/A & 0.07 & N/A & 0.07 & N/A & 0.07 & N/A \\
\hline $\mathrm{Fe}_{2} \mathrm{O}_{3}$ & 5.28 & 5.33 & 5.28 & 5.16 & 5.28 & 5.47 & 0.00 & 0.07 & 0.00 & 0.16 & 0.00 & 0.07 & 0.00 & 0.09 & 0.00 & 0.09 \\
\hline $\mathrm{K}_{2} \mathrm{O}$ & 0.26 & 0.37 & 0.26 & 0.32 & 0.26 & 0.30 & 0.26 & 0.32 & 0.26 & 0.31 & 0.26 & 0.30 & 0.26 & 0.37 & 0.26 & 0.34 \\
\hline $\mathrm{Li}_{2} \mathrm{O}$ & 5.79 & N/A & 5.79 & N/A & 5.79 & N/A & 5.79 & N/A & 5.79 & N/A & 5.79 & N/A & 5.03 & N/A & 5.03 & N/A \\
\hline $\mathrm{MgO}$ & 2.96 & 2.96 & 2.96 & 2.94 & 2.96 & 2.85 & 2.96 & 2.79 & 2.96 & 2.92 & 2.96 & 2.72 & 2.96 & 2.84 & 2.96 & 2.86 \\
\hline $\mathrm{Na}_{2} \mathrm{O}$ & 5.47 & 5.69 & 5.47 & 5.36 & 5.47 & 4.99 & 5.47 & 5.66 & 5.47 & 5.37 & 5.47 & 4.63 & 5.47 & 5.82 & 5.47 & 5.81 \\
\hline $\mathrm{P}_{2} \mathrm{O}_{5}$ & 0.01 & 0.02 & 0.01 & 0.02 & 0.01 & $\mathrm{BDL}$ & 0.01 & $\mathrm{BDL}$ & 0.01 & 0.03 & 0.01 & 0.03 & 0.01 & 0.02 & 0.01 & 0.03 \\
\hline $\mathrm{SiO}_{2}$ & 48.35 & 49.81 & 48.35 & 50.02 & 48.35 & 49.88 & 48.35 & 49.85 & 48.35 & 49.54 & 48.35 & 50.06 & 48.73 & 49.78 & 48.73 & 50.88 \\
\hline $\mathrm{SO}_{3}$ & 1.28 & 0.71 & 4.50 & 1.54 & 4.50 & 1.43 & 1.28 & 0.89 & 4.50 & 1.94 & 4.50 & 1.83 & 1.28 & 0.84 & 0.00 & 0.01 \\
\hline $\mathrm{TiO}_{2}$ & 1.39 & 1.42 & 1.39 & 1.45 & 1.39 & 1.52 & 1.39 & 1.46 & 1.39 & 1.46 & 1.39 & 1.51 & 1.39 & 1.46 & 1.39 & 1.42 \\
\hline $\mathrm{ZnO}$ & 3.15 & 2.94 & 3.15 & 2.80 & 3.15 & 2.95 & 3.15 & 3.02 & 3.15 & 2.94 & 3.15 & 3.01 & 5.79 & 5.60 & 5.79 & 5.26 \\
\hline $\mathrm{ZrO}_{2}$ & 3.15 & 3.25 & 3.15 & 3.28 & 3.15 & 3.61 & 3.15 & 3.45 & 3.15 & 3.41 & 3.15 & 3.65 & 3.15 & 3.55 & 3.15 & 3.35 \\
\hline Sum* & 100.00 & 101.10 & 103.20 & 101.20 & 103.20 & 101.10 & 100.00 & 101.10 & 103.20 & 101.30 & 103.20 & 101.20 & 100.00 & 101.20 & 98.70 & 101.10 \\
\hline
\end{tabular}


Table 4.8. XRF Analysis of Glasses Tested for LAW AZ-102 (cont'd).

\begin{tabular}{|c|c|c|c|c|c|c|c|c|c|c|c|c|c|c|c|c|}
\hline \multirow{2}{*}{$\begin{array}{c}\text { Oxide } \\
- \\
\end{array}$} & \multicolumn{2}{|c|}{ LAWB63S0R1 } & \multicolumn{2}{|c|}{ LAWB63S0R2 } & \multicolumn{2}{|c|}{ LAWB63S0R3 } & \multicolumn{2}{|c|}{ LAWB63S4 } & \multicolumn{2}{|c|}{ LAWB63S4W } & \multicolumn{2}{|c|}{ LAWB64 } & \multicolumn{2}{|c|}{ LAWB64S0 } & \multicolumn{2}{|c|}{ LAWB64S4 } \\
\hline & Target & XRF & Target & XRF & Target & XRF & Target & XRF & Target & XRF & Target & XRF & Target & XRF & Target & XRF \\
\hline $\mathrm{Al}_{2} \mathrm{O}_{3}$ & 6.55 & 6.50 & 6.55 & 6.79 & 6.55 & 6.80 & 6.55 & 6.59 & 6.55 & 6.53 & 6.17 & 6.05 & 6.17 & 6.22 & 6.17 & 6.23 \\
\hline $\mathrm{B}_{2} \mathrm{O}_{3}$ & 9.91 & N/A & 9.91 & N/A & 9.91 & N/A & 9.91 & N/A & 9.91 & N/A & 9.91 & N/A & 9.91 & N/A & 9.91 & N/A \\
\hline $\mathrm{CaO}$ & 9.31 & 9.46 & 9.31 & 9.44 & 9.31 & 9.47 & 9.31 & 9.02 & 9.31 & 8.88 & 6.67 & 6.72 & 6.67 & 6.69 & 6.67 & 6.54 \\
\hline $\mathrm{Cl}$ & 0.00 & 0.00 & 0.00 & 0.01 & 0.00 & $\mathrm{BDL}$ & 0.00 & $\mathrm{BDL}$ & 0.00 & 0.00 & 0.00 & $\mathrm{BDL}$ & 0.00 & 0.01 & 0.00 & 0.00 \\
\hline $\mathrm{Cr}_{2} \mathrm{O}_{3}$ & 0.10 & 0.10 & 0.10 & 0.10 & 0.10 & 0.10 & 0.10 & 0.09 & 0.10 & 0.10 & 0.10 & 0.10 & 0.10 & 0.10 & 0.10 & 0.10 \\
\hline $\mathrm{F}$ & 0.07 & N/A & 0.07 & N/A & 0.07 & N/A & 0.07 & N/A & 0.07 & N/A & 0.07 & N/A & 0.07 & N/A & 0.07 & N/A \\
\hline $\mathrm{Fe}_{2} \mathrm{O}_{3}$ & 0.00 & 0.04 & 0.00 & 0.06 & 0.00 & 0.05 & 0.00 & 0.04 & 0.00 & 0.03 & 3.28 & 3.47 & 3.28 & 3.53 & 3.28 & 3.45 \\
\hline $\mathrm{K}_{2} \mathrm{O}$ & 0.26 & 0.33 & 0.26 & 0.34 & 0.26 & 0.34 & 0.26 & 0.31 & 0.26 & 0.30 & 0.26 & 0.35 & 0.26 & 0.34 & 0.26 & 0.32 \\
\hline $\mathrm{Li}_{2} \mathrm{O}$ & 5.03 & N/A & 5.03 & N/A & 5.03 & N/A & 5.03 & N/A & 5.03 & N/A & 5.79 & N/A & 5.79 & N/A & 5.79 & N/A \\
\hline $\mathrm{MgO}$ & 2.96 & 2.78 & 2.96 & 3.01 & 2.96 & 2.96 & 2.96 & 2.81 & 2.96 & 2.79 & 2.96 & 2.79 & 2.96 & 2.97 & 2.96 & 2.90 \\
\hline $\mathrm{Na}_{2} \mathrm{O}$ & 5.47 & 6.02 & 5.47 & 5.69 & 5.47 & 5.61 & 5.47 & 5.35 & 5.47 & 4.99 & 5.47 & 5.95 & 5.47 & 6.17 & 5.47 & 5.04 \\
\hline $\mathrm{P}_{2} \mathrm{O}_{5}$ & 0.01 & 0.02 & 0.01 & 0.02 & 0.01 & 0.02 & 0.01 & BDL & 0.01 & 0.02 & 0.01 & $\mathrm{BDL}$ & 0.01 & 0.02 & 0.01 & 0.02 \\
\hline $\mathrm{SiO}_{2}$ & 48.73 & 50.13 & 48.73 & 49.75 & 48.73 & 49.94 & 48.73 & 50.10 & 48.73 & 50.67 & 48.35 & 49.45 & 48.35 & 49.78 & 48.35 & 49.33 \\
\hline $\mathrm{SO}_{3}$ & 0.00 & 0.01 & 0.00 & BDL & 0.00 & $\mathrm{BDL}$ & 4.50 & 1.55 & 4.50 & 1.43 & 1.28 & 0.68 & 0.00 & 0.01 & 4.50 & 1.80 \\
\hline $\mathrm{TiO}_{2}$ & 1.39 & 1.56 & 1.39 & 1.57 & 1.39 & 1.57 & 1.39 & 1.51 & 1.39 & 1.53 & 1.39 & 1.49 & 1.39 & 1.68 & 1.39 & 1.53 \\
\hline $\mathrm{ZnO}$ & 5.79 & 5.71 & 5.79 & 5.74 & 5.79 & 5.70 & 5.79 & 5.50 & 5.79 & 5.54 & 5.15 & 4.98 & 5.15 & 5.07 & 5.15 & 4.92 \\
\hline $\mathrm{ZrO}_{2}$ & 3.15 & 3.47 & 3.15 & 3.70 & 3.15 & 3.68 & 3.15 & 3.54 & 3.15 & 3.55 & 3.15 & 3.43 & 3.15 & 3.70 & 3.15 & 3.46 \\
\hline Sum* & 98.70 & 101.10 & 98.70 & 101.10 & 98.70 & 101.20 & 103.20 & 101.30 & 103.20 & 101.30 & 100.00 & 101.20 & 98.70 & 102.00 & 103.20 & 101.30 \\
\hline
\end{tabular}

$\mathrm{BDL}=$ Below detection limit of the instrument; N/A = Not Analyzed; Target value used for $\mathrm{B}_{2} \mathrm{O}_{3}$ and $\mathrm{Li}_{2} \mathrm{O}$; * $=$ Includes $\mathrm{B}_{2} \mathrm{O}_{3}$ and $\mathrm{Li}_{2} \mathrm{O}$ in Sum. 
Table 4.8. XRF Analysis of Glasses Tested for LAW AZ-102 (cont'd).

\begin{tabular}{|c|c|c|c|c|c|c|c|c|c|c|c|c|c|c|c|c|}
\hline \multirow{2}{*}{$\begin{array}{c}\text { Oxide } \\
-\end{array}$} & \multicolumn{2}{|c|}{ LAWB64S4W } & \multicolumn{2}{|c|}{ LAWB65 } & \multicolumn{2}{|c|}{ LAWB65S4 } & \multicolumn{2}{|c|}{ LAWB65S4W } & \multicolumn{2}{|c|}{ LAWB66 } & \multicolumn{2}{|c|}{ LAWB66S4 } & \multicolumn{2}{|c|}{ LAWB66S4W } & \multicolumn{2}{|c|}{ LAWB67 } \\
\hline & Target & XRF & Target & XRF & Target & XRF & Target & XRF & Target & XRF & Target & XRF & Target & XRF & Target & XRF \\
\hline $\mathrm{Al}_{2} \mathrm{O}_{3}$ & 6.17 & 6.05 & 6.17 & 6.27 & 6.17 & 6.30 & 6.17 & 6.05 & 6.17 & 6.24 & 6.17 & 6.35 & 6.17 & 6.18 & 6.17 & 6.38 \\
\hline $\mathrm{B}_{2} \mathrm{O}_{3}$ & 9.91 & N/A & 9.91 & N/A & 9.91 & N/A & 9.91 & N/A & 9.91 & N/A & 9.91 & N/A & 9.91 & N/A & 9.91 & N/A \\
\hline $\mathrm{CaO}$ & 6.67 & 6.52 & 6.67 & 6.54 & 6.67 & 6.41 & 6.67 & 6.28 & 8.17 & 8.00 & 8.17 & 7.87 & 8.17 & 7.80 & 5.17 & 5.12 \\
\hline $\mathrm{Cl}$ & 0.00 & $\mathrm{BDL}$ & 0.00 & 0.01 & 0.00 & $\mathrm{BDL}$ & 0.00 & $\mathrm{BDL}$ & 0.00 & BDL & 0.00 & 0.00 & 0.00 & $\mathrm{BDL}$ & 0.00 & $\mathrm{BDL}$ \\
\hline $\mathrm{Cr}_{2} \mathrm{O}_{3}$ & 0.10 & 0.10 & 0.10 & 0.09 & 0.10 & 0.10 & 0.10 & 0.10 & 0.10 & 0.10 & 0.10 & 0.08 & 0.10 & 0.09 & 0.10 & 0.09 \\
\hline $\mathrm{F}$ & 0.07 & N/A & 0.07 & N/A & 0.07 & N/A & 0.07 & N/A & 0.07 & N/A & 0.07 & N/A & 0.07 & N/A & 0.07 & N/A \\
\hline $\mathrm{Fe}_{2} \mathrm{O}_{3}$ & 3.28 & 3.61 & 5.28 & 5.32 & 5.28 & 5.41 & 5.28 & 5.57 & 5.28 & 5.40 & 5.28 & 5.30 & 5.28 & 5.45 & 5.28 & 5.38 \\
\hline $\mathrm{K}_{2} \mathrm{O}$ & 0.26 & 0.30 & 0.26 & 0.36 & 0.26 & 0.33 & 0.26 & 0.33 & 0.26 & 0.35 & 0.26 & 0.32 & 0.26 & 0.32 & 0.26 & 0.36 \\
\hline $\mathrm{Li}_{2} \mathrm{O}$ & 5.79 & N/A & 4.29 & N/A & 4.29 & N/A & 4.29 & N/A & 4.29 & N/A & 4.29 & N/A & 4.29 & N/A & 4.29 & N/A \\
\hline $\mathrm{MgO}$ & 2.96 & 2.73 & 2.96 & 2.89 & 2.96 & 2.88 & 2.96 & 2.65 & 2.96 & 3.02 & 2.96 & 2.96 & 2.96 & 2.71 & 2.96 & 2.84 \\
\hline $\mathrm{Na}_{2} \mathrm{O}$ & 5.47 & 4.57 & 5.47 & 5.86 & 5.47 & 5.05 & 5.47 & 4.29 & 5.47 & 5.63 & 5.47 & 5.29 & 5.47 & 4.75 & 5.47 & 5.56 \\
\hline $\mathrm{P}_{2} \mathrm{O}_{5}$ & 0.01 & 0.02 & 0.01 & 0.02 & 0.01 & 0.02 & 0.01 & 0.02 & 0.01 & 0.02 & 0.01 & 0.02 & 0.01 & 0.02 & 3.01 & 3.44 \\
\hline $\mathrm{SiO}_{2}$ & 48.35 & 49.74 & 48.35 & 49.45 & 48.35 & 49.18 & 48.35 & 50.63 & 48.35 & 49.62 & 48.35 & 49.46 & 48.35 & 50.17 & 48.35 & 49.94 \\
\hline $\mathrm{SO}_{3}$ & 4.50 & 1.40 & 1.28 & 0.89 & 4.50 & 2.10 & 4.50 & 1.26 & 1.28 & 0.65 & 4.50 & 1.72 & 4.50 & 1.35 & 1.28 & 0.97 \\
\hline $\mathrm{TiO}_{2}$ & 1.39 & 1.57 & 1.39 & 1.45 & 1.39 & 1.46 & 1.39 & 1.52 & 1.39 & 1.44 & 1.39 & 1.38 & 1.39 & 1.44 & 1.39 & 1.44 \\
\hline $\mathrm{ZnO}$ & 5.15 & 5.10 & 4.65 & 4.33 & 4.65 & 4.39 & 4.65 & 4.49 & 3.15 & 2.97 & 3.15 & 2.97 & 3.15 & 2.98 & 3.15 & 2.97 \\
\hline $\mathrm{ZrO}_{2}$ & 3.15 & 3.82 & 3.15 & 3.45 & 3.15 & 3.44 & 3.15 & 3.70 & 3.15 & 3.43 & 3.15 & 3.25 & 3.15 & 3.62 & 3.15 & 3.42 \\
\hline Sum* & 103.20 & 101.20 & 100.00 & 101.10 & 103.20 & 101.30 & 103.20 & 101.10 & 100.00 & 101.10 & 103.20 & 101.20 & 103.20 & 101.10 & 100.00 & 102.10 \\
\hline
\end{tabular}

$\mathrm{BDL}=$ Below detection limit of the instrument; N/A = Not Analyzed; Target value used for $\mathrm{B}_{2} \mathrm{O}_{3}$ and $\mathrm{Li}_{2} \mathrm{O}$; * = Includes $\mathrm{B}_{2} \mathrm{O}_{3}$ and $\mathrm{Li}_{2} \mathrm{O}$ in $\mathrm{Sum}_{\text {. }}$ 
Table 4.8. XRF Analysis of Glasses Tested for LAW AZ-102 (cont'd).

\begin{tabular}{|c|c|c|c|c|c|c|c|c|c|c|c|c|c|c|c|c|}
\hline \multirow{2}{*}{$\begin{array}{c}\text { Oxide } \\
-\end{array}$} & \multicolumn{2}{|c|}{ LAWB67S4 } & \multicolumn{2}{|c|}{ LAWB67S4W } & \multicolumn{2}{|c|}{ LAWB68 } & \multicolumn{2}{|c|}{ LAWB73 } & \multicolumn{2}{|c|}{ LAWB73S4 } & \multicolumn{2}{|c|}{ LAWB73S4W } & \multicolumn{2}{|c|}{ LAWB73S0 } & \multicolumn{2}{|c|}{ LAWB74 } \\
\hline & Target & $\mathrm{XRF}$ & Target & XRF & Target & XRF & Target & XRF & Target & XRF & Target & XRF & Target & XRF & Target & XRF \\
\hline $\mathrm{Al}_{2} \mathrm{O}_{3}$ & 6.17 & 6.20 & 6.17 & 5.90 & 6.17 & 6.09 & 6.17 & 5.62 & 6.17 & 6.33 & 6.17 & 6.07 & 6.17 & 5.85 & 6.17 & 6.32 \\
\hline $\mathrm{B}_{2} \mathrm{O}_{3}$ & 9.91 & N/A & 9.91 & N/A & 8.41 & N/A & 9.91 & N/A & 9.91 & N/A & 9.91 & N/A & 9.91 & N/A & 10.03 & N/A \\
\hline $\mathrm{CaO}$ & 5.17 & 4.64 & 5.17 & 4.63 & 8.17 & 8.28 & 9.31 & 9.26 & 9.31 & 8.94 & 9.31 & 9.09 & 9.31 & 9.13 & 8.66 & 8.65 \\
\hline $\mathrm{Cl}$ & 0.00 & 0.01 & 0.00 & 0.00 & 0.00 & $\mathrm{BDL}$ & 0.00 & 0.01 & 0.00 & $\mathrm{BDL}$ & 0.00 & $\mathrm{BDL}$ & 0.00 & 0.01 & 0.00 & $\mathrm{BDL}$ \\
\hline $\mathrm{Cr}_{2} \mathrm{O}_{3}$ & 0.10 & 0.08 & 0.10 & 0.09 & 0.10 & 0.09 & 0.10 & 0.40 & 0.10 & 0.09 & 0.10 & 0.11 & 0.10 & 0.09 & 0.10 & 0.11 \\
\hline $\mathrm{F}$ & 0.07 & N/A & 0.07 & N/A & 0.07 & N/A & 0.07 & N/A & 0.07 & N/A & 0.07 & N/A & 0.07 & N/A & 0.07 & N/A \\
\hline $\mathrm{Fe}_{2} \mathrm{O}_{3}$ & 5.28 & 4.97 & 5.28 & 5.18 & 5.28 & 5.68 & 1.90 & 2.78 & 1.90 & 2.02 & 1.90 & 2.18 & 1.90 & 2.02 & 1.90 & 2.07 \\
\hline $\mathrm{K}_{2} \mathrm{O}$ & 0.26 & 0.31 & 0.26 & 0.32 & 0.26 & 0.36 & 0.26 & 0.31 & 0.26 & 0.30 & 0.26 & 0.29 & 0.26 & 0.31 & 0.26 & 0.35 \\
\hline $\mathrm{Li}_{2} \mathrm{O}$ & 4.29 & N/A & 4.29 & N/A & 4.29 & N/A & 5.03 & N/A & 5.03 & N/A & 5.03 & N/A & 5.03 & N/A & 5.29 & N/A \\
\hline $\mathrm{MgO}$ & 2.96 & 5.62 & 2.96 & 5.13 & 2.96 & 2.81 & 2.96 & 2.83 & 2.96 & 2.83 & 2.96 & 2.73 & 2.96 & 2.92 & 2.96 & 2.83 \\
\hline $\mathrm{Na}_{2} \mathrm{O}$ & 5.47 & 5.95 & 5.47 & 5.25 & 5.47 & 5.32 & 5.47 & 6.00 & 5.47 & 5.02 & 5.47 & 4.66 & 5.47 & 5.18 & 5.47 & 5.45 \\
\hline $\mathrm{P}_{2} \mathrm{O}_{5}$ & 3.01 & 3.17 & 3.01 & 3.26 & 0.01 & 0.06 & 0.01 & 0.02 & 0.01 & BDL & 0.01 & 0.02 & 0.01 & 0.02 & 0.01 & 0.02 \\
\hline $\mathrm{SiO}_{2}$ & 48.35 & 48.64 & 48.35 & 49.43 & 48.35 & 49.02 & 48.35 & 48.01 & 48.35 & 50.16 & 48.35 & 49.65 & 48.35 & 51.11 & 48.35 & 49.50 \\
\hline $\mathrm{SO}_{3}$ & 4.50 & 1.60 & 4.50 & 1.42 & 1.28 & 0.83 & 1.28 & 0.90 & 4.50 & 1.51 & 4.50 & 1.40 & 0.00 & 0.01 & 1.28 & 0.77 \\
\hline $\mathrm{TiO}_{2}$ & 1.39 & 1.30 & 1.39 & 1.38 & 1.39 & 1.50 & 1.39 & 1.47 & 1.39 & 1.42 & 1.39 & 1.50 & 1.39 & 1.42 & 1.39 & 1.47 \\
\hline $\mathrm{ZnO}$ & 3.15 & 2.72 & 3.15 & 2.79 & 4.65 & 4.67 & 4.65 & 4.73 & 4.65 & 4.45 & 4.65 & 4.73 & 4.65 & 4.41 & 4.65 & 4.48 \\
\hline $\mathrm{ZrO}_{2}$ & 3.15 & 3.11 & 3.15 & 3.39 & 3.15 & 3.65 & 3.15 & 3.77 & 3.15 & 3.24 & 3.15 & 3.85 & 3.15 & 3.54 & 3.15 & 3.58 \\
\hline Sum* & 103.20 & 102.50 & 103.20 & 102.40 & 100.00 & 101.00 & 98.70 & 101.04 & 100.00 & 101.25 & 100.00 & 101.20 & 103.20 & 100.95 & 103.20 & 101.19 \\
\hline
\end{tabular}

$\mathrm{BDL}=$ Below detection limit of the instrument; N/A = Not Analyzed; Target value used for $\mathrm{B}_{2} \mathrm{O}_{3}$ and $\mathrm{Li}_{2} \mathrm{O}$; * $=$ Includes $\mathrm{B}_{2} \mathrm{O}_{3}$ and $\mathrm{Li}_{2} \mathrm{O}$ in Sum. 
Table 4.8. XRF Analysis of Glasses Tested for LAW AZ-102 (cont'd).

\begin{tabular}{|c|c|c|c|c|c|c|c|c|c|c|c|c|c|c|c|c|}
\hline \multirow{2}{*}{$\begin{array}{c}\text { Oxide } \\
-\end{array}$} & \multicolumn{2}{|c|}{ LAWB74S4 } & \multicolumn{2}{|c|}{ LAWB74S4W } & \multicolumn{2}{|c|}{ LAWB74S0 } & \multicolumn{2}{|c|}{ LAWB75 } & \multicolumn{2}{|c|}{ LAWB75S0 } & \multicolumn{2}{|c|}{ LAWB75S4 } & \multicolumn{2}{|c|}{ LAWB75S4W } & \multicolumn{2}{|c|}{ LAWB76 } \\
\hline & Target & $\mathrm{XRF}$ & Target & XRF & Target & XRF & Target & XRF & Target & XRF & Target & XRF & Target & XRF & Target & XRF \\
\hline $\mathrm{Al}_{2} \mathrm{O}_{3}$ & 6.17 & 6.38 & 6.17 & 6.09 & 6.17 & 5.98 & 6.17 & 6.08 & 6.17 & 6.45 & 6.17 & 6.42 & 6.17 & 5.98 & 6.17 & 6.35 \\
\hline $\mathrm{B}_{2} \mathrm{O}_{3}$ & 10.30 & N/A & 10.30 & N/A & 10.30 & N/A & 11.76 & N/A & 11.76 & N/A & 11.76 & N/A & 11.76 & N/A & 11.76 & N/A \\
\hline $\mathrm{CaO}$ & 8.66 & 8.23 & 8.66 & 8.45 & 8.66 & 8.34 & 8.66 & 8.57 & 8.66 & 8.73 & 8.66 & 8.13 & 8.66 & 8.55 & 8.66 & 8.34 \\
\hline $\mathrm{Cl}$ & 0.00 & $\mathrm{BDL}$ & 0.00 & $\mathrm{BDL}$ & 0.00 & 0.01 & 0.00 & $\mathrm{BDL}$ & 0.00 & 0.01 & 0.00 & $\mathrm{BDL}$ & 0.00 & $\mathrm{BDL}$ & 0.00 & 0.01 \\
\hline $\mathrm{Cr}_{2} \mathrm{O}_{3}$ & 0.10 & 0.09 & 0.10 & 0.10 & 0.10 & 0.09 & 0.10 & 0.10 & 0.10 & 0.10 & 0.10 & 0.10 & 0.10 & 0.11 & 0.10 & 0.09 \\
\hline $\mathrm{F}$ & 0.07 & N/A & 0.07 & N/A & 0.07 & N/A & 0.07 & N/A & 0.07 & N/A & 0.07 & N/A & 0.07 & N/A & 0.07 & N/A \\
\hline $\mathrm{Fe}_{2} \mathrm{O}_{3}$ & 1.90 & 1.97 & 1.90 & 2.13 & 1.90 & 1.97 & 1.90 & 2.01 & 1.90 & 2.07 & 1.90 & 1.94 & 1.90 & 2.17 & 1.90 & 1.91 \\
\hline $\mathrm{K}_{2} \mathrm{O}$ & 0.26 & 0.29 & 0.26 & 0.28 & 0.26 & 0.32 & 0.26 & 0.33 & 0.26 & 0.35 & 0.26 & 0.30 & 0.26 & 0.30 & 0.26 & 0.34 \\
\hline $\mathrm{Li}_{2} \mathrm{O}$ & 5.29 & N/A & 5.29 & N/A & 5.29 & N/A & 5.29 & N/A & 5.29 & N/A & 5.29 & N/A & 5.29 & N/A & 5.79 & N/A \\
\hline $\mathrm{MgO}$ & 2.96 & 2.99 & 2.96 & 2.83 & 2.96 & 2.79 & 1.50 & 1.40 & 1.50 & 1.43 & 1.50 & 1.44 & 1.50 & 1.38 & 1.50 & 1.45 \\
\hline $\mathrm{Na}_{2} \mathrm{O}$ & 5.47 & 5.24 & 5.47 & 4.62 & 5.47 & 5.39 & 5.47 & 5.72 & 5.47 & 5.59 & 5.47 & 5.33 & 5.47 & 4.90 & 5.47 & 6.41 \\
\hline $\mathrm{P}_{2} \mathrm{O}_{5}$ & 0.01 & 0.02 & 0.01 & 0.02 & 0.01 & BDL & 0.01 & 0.02 & 0.01 & 0.02 & 0.01 & 0.02 & 0.01 & BDL & 0.01 & 0.02 \\
\hline $\mathrm{SiO}_{2}$ & 48.35 & 50.06 & 48.35 & 49.81 & 48.35 & 51.29 & 48.35 & 49.37 & 48.35 & 49.91 & 48.35 & 50.15 & 48.35 & 49.06 & 49.24 & 49.90 \\
\hline $\mathrm{SO}_{3}$ & 4.50 & 1.46 & 4.50 & 1.43 & 0.00 & 0.01 & 1.28 & 1.00 & 0.00 & 0.20 & 4.50 & 1.54 & 4.50 & 1.47 & 1.28 & 0.95 \\
\hline $\mathrm{TiO}_{2}$ & 1.39 & 1.43 & 1.39 & 1.51 & 1.39 & 1.39 & 1.39 & 1.41 & 1.39 & 1.44 & 1.39 & 1.40 & 1.39 & 1.50 & 0.00 & 0.03 \\
\hline $\mathrm{ZnO}$ & 4.65 & 4.33 & 4.65 & 4.64 & 4.65 & 4.32 & 4.65 & 4.44 & 4.65 & 4.54 & 4.65 & 4.25 & 4.65 & 4.81 & 4.65 & 4.30 \\
\hline $\mathrm{ZrO}_{2}$ & 3.15 & 3.20 & 3.15 & 3.77 & 3.15 & 3.46 & 3.15 & 3.55 & 3.15 & 3.57 & 3.15 & 3.09 & 3.15 & 3.85 & 3.15 & 3.51 \\
\hline Sum* & 103.20 & 101.30 & 103.20 & 101.30 & 98.70 & 100.90 & 103.20 & 101.04 & 100.00 & 100.95 & 100.00 & 101.15 & 98.70 & 101.11 & 103.20 & 101.15 \\
\hline
\end{tabular}

$\mathrm{BDL}=$ Below detection limit of the instrument; N/A = Not Analyzed; Target value used for $\mathrm{B}_{2} \mathrm{O}_{3}$ and $\mathrm{Li}_{2} \mathrm{O}$; * $=$ Includes $\mathrm{B}_{2} \mathrm{O}_{3}$ and $\mathrm{Li}_{2} \mathrm{O}$ in Sum. 
Table 4.8. XRF Analysis of Glasses Tested for LAW AZ-102 (cont'd).

\begin{tabular}{|c|c|c|c|c|c|c|c|c|c|c|c|c|}
\hline Oxide & \multicolumn{2}{|c|}{ LAWB76-2 } & \multicolumn{2}{|c|}{ LAWB76-3 } & \multicolumn{2}{|c|}{ LAWB76-4 } & \multicolumn{2}{|c|}{ LAWB76S0 } & \multicolumn{2}{|c|}{ LAWB76S4 } & \multicolumn{2}{|c|}{ LAWB76S4W } \\
\hline- & Target & XRF & Target & XRF & Target & XRF & Target & XRF & Target & XRF & Target & XRF \\
\hline $\mathrm{Al}_{2} \mathrm{O}_{3}$ & 6.17 & 5.84 & 6.17 & 6.23 & 6.17 & 5.73 & 6.17 & 6.30 & 6.17 & 6.29 & 6.17 & 6.12 \\
\hline $\mathrm{B}_{2} \mathrm{O}_{3}$ & 11.76 & N/A & 11.76 & N/A & 11.76 & N/A & 11.76 & N/A & 11.76 & N/A & 11.76 & N/A \\
\hline $\mathrm{CaO}$ & 8.66 & 8.36 & 8.66 & 8.58 & 8.66 & 8.53 & 8.66 & 8.88 & 8.66 & 8.47 & 8.66 & 8.62 \\
\hline $\mathrm{Cl}$ & 0.00 & BDL & 0.00 & BDL & 0.00 & 0.01 & 0.00 & 0.01 & 0.00 & 0.00 & 0.00 & BDL \\
\hline $\mathrm{Cr}_{2} \mathrm{O}_{3}$ & 0.10 & 0.32 & 0.10 & 0.09 & 0.10 & 0.37 & 0.10 & 0.10 & 0.10 & 0.11 & 0.10 & 0.11 \\
\hline $\mathrm{F}$ & 0.07 & N/A & 0.07 & N/A & 0.07 & N/A & 0.07 & N/A & 0.07 & N/A & 0.07 & N/A \\
\hline $\mathrm{Fe}_{2} \mathrm{O}_{3}$ & 1.90 & 2.48 & 1.90 & 2.00 & 1.90 & 2.64 & 1.90 & 2.06 & 1.90 & 1.97 & 1.90 & 2.13 \\
\hline $\mathrm{K}_{2} \mathrm{O}$ & 0.26 & 0.32 & 0.26 & 0.32 & 0.26 & 0.31 & 0.26 & 0.34 & 0.26 & 0.29 & 0.26 & 0.29 \\
\hline $\mathrm{Li}_{2} \mathrm{O}$ & 5.79 & N/A & 5.79 & N/A & 5.79 & N/A & 5.79 & N/A & 5.79 & N/A & 5.79 & N/A \\
\hline $\mathrm{MgO}$ & 1.50 & 1.42 & 1.50 & 1.45 & 1.50 & 1.48 & 1.50 & 1.32 & 1.50 & 1.41 & 1.50 & 1.38 \\
\hline $\mathrm{Na}_{2} \mathrm{O}$ & 5.47 & 6.36 & 5.47 & 5.88 & 5.47 & 6.06 & 5.47 & 5.21 & 5.47 & 4.92 & 5.47 & 4.50 \\
\hline $\mathrm{P}_{2} \mathrm{O}_{5}$ & 0.01 & 0.01 & 0.01 & BDL & 0.01 & 0.01 & 0.01 & 0.02 & 0.01 & BDL & 0.01 & $\mathrm{BDL}$ \\
\hline $\mathrm{SiO}_{2}$ & 49.24 & 49.52 & 49.24 & 49.88 & 49.24 & 49.30 & 49.24 & 50.87 & 49.24 & 51.14 & 49.24 & 50.30 \\
\hline $\mathrm{SO}_{3}$ & 1.28 & 0.90 & 1.28 & 0.89 & 1.28 & 0.89 & 0.00 & 0.06 & 4.50 & 1.71 & 4.50 & 1.58 \\
\hline $\mathrm{TiO}_{2}$ & 0.00 & 0.02 & 0.00 & 0.03 & 0.00 & 0.02 & 0.00 & 0.02 & 0.00 & 0.02 & 0.00 & 0.02 \\
\hline $\mathrm{ZnO}$ & 4.65 & 4.36 & 4.65 & 4.52 & 4.65 & 4.44 & 4.65 & 4.63 & 4.65 & 4.42 & 4.65 & 4.74 \\
\hline $\mathrm{ZrO}_{2}$ & 3.15 & 3.54 & 3.15 & 3.70 & 3.15 & 3.62 & 3.15 & 3.62 & 3.15 & 3.38 & 3.15 & 3.87 \\
\hline Sum* & 100.00 & 101.00 & 100.00 & 101.10 & 100.00 & 101.00 & 98.70 & 101.00 & 103.20 & 101.20 & 103.20 & 101.20 \\
\hline
\end{tabular}

$\mathrm{BDL}=$ Below detection limit of the instrument; N/A = Not Analyzed; Target value used for $\mathrm{B}_{2} \mathrm{O}_{3}$ and $\mathrm{Li}_{2} \mathrm{O}$; * $=$ Includes $\mathrm{B}_{2} \mathrm{O}_{3}$ and $\mathrm{Li}_{2} \mathrm{O}$ in Sum. 
Table 4.9 XRF Analysis of Glasses Tested for LAW AN-107.

\begin{tabular}{|c|c|c|}
\hline Oxide & \multicolumn{2}{|c|}{ C22AN107 } \\
\hline - & Target & XRF \\
\hline $\mathrm{Al}_{2} \mathrm{O}_{3}$ & 6.10 & 5.53 \\
\hline $\mathrm{B}_{2} \mathrm{O}_{3}$ & 10.07 & N/A \\
\hline $\mathrm{CaO}$ & 5.11 & 5.16 \\
\hline $\mathrm{Cl}$ & 0.08 & 0.08 \\
\hline $\mathrm{Cr}_{2} \mathrm{O}_{3}$ & 0.02 & 0.36 \\
\hline $\mathrm{F}$ & 0.15 & N/A \\
\hline $\mathrm{Fe}_{2} \mathrm{O}_{3}$ & 5.59 & 6.89 \\
\hline $\mathrm{K}_{2} \mathrm{O}$ & 0.09 & 0.17 \\
\hline $\mathrm{Li}_{2} \mathrm{O}$ & 2.51 & N/A \\
\hline $\mathrm{MgO}$ & 1.51 & 1.33 \\
\hline $\mathrm{MoO}_{3}$ & 0.00 & 0.01 \\
\hline $\mathrm{Na}_{2} \mathrm{O}$ & 14.42 & 13.90 \\
\hline $\mathrm{NiO}$ & 0.03 & 0.10 \\
\hline $\mathrm{P}_{2} \mathrm{O}_{5}$ & 0.12 & 0.19 \\
\hline $\mathrm{PbO}$ & 0.02 & 0.02 \\
\hline $\mathrm{Re}_{2} \mathrm{O}_{7}$ & 0.10 & 0.06 \\
\hline $\mathrm{SiO}_{2}$ & 46.57 & 46.13 \\
\hline $\mathrm{SO}_{3}$ & 0.31 & 0.27 \\
\hline $\mathrm{SrO}$ & 0.00 & 0.01 \\
\hline $\mathrm{TiO}_{2}$ & 1.14 & 1.20 \\
\hline $\mathrm{ZnO}$ & 3.06 & 3.17 \\
\hline $\mathrm{ZrO}_{2}$ & 3.02 & 3.60 \\
\hline Sum* & 100.00 & 100.80 \\
\hline
\end{tabular}

$\mathrm{BDL}=$ Below detection limit of the instrument; N/A = Not Analyzed;

Target value used for $\mathrm{B}_{2} \mathrm{O}_{3}$ and $\mathrm{Li}_{2} \mathrm{O}$; * = Includes $\mathrm{B}_{2} \mathrm{O}_{3}$ and $\mathrm{Li}_{2} \mathrm{O}$ in Sum. 
Table 4.10. XRF Analysis of Glasses Tested for LAW AN-102.

\begin{tabular}{|c|c|c|c|c|c|c|c|c|c|c|c|c|c|c|c|c|}
\hline \multirow{2}{*}{$\begin{array}{c}\text { Oxide } \\
- \\
\end{array}$} & \multicolumn{2}{|c|}{ C21REV2 } & \multicolumn{2}{|c|}{ C21REV2S0 } & \multicolumn{2}{|c|}{ C21REV2S2 } & \multicolumn{2}{|c|}{ LAWC26 } & \multicolumn{2}{|c|}{ LAWC26S2 } & \multicolumn{2}{|c|}{ LAWC26S2W } & \multicolumn{2}{|c|}{ LAWC27 } & \multicolumn{2}{|c|}{ LAWC27S2 } \\
\hline & Target & XRF & Target & XRF & Target & XRF & Target & $\mathrm{XRF}$ & Target & $\mathrm{XRF}$ & Target & $\mathrm{XRF}$ & Target & $\mathrm{XRF}$ & Target & XRF \\
\hline $\mathrm{Al}_{2} \mathrm{O}_{3}$ & 6.12 & 6.16 & 6.12 & 6.04 & 6.12 & 6.11 & 6.12 & 6.16 & 6.13 & 6.83 & 6.13 & 6.71 & 6.12 & 6.18 & 6.13 & 6.12 \\
\hline $\mathrm{B}_{2} \mathrm{O}_{3}$ & 10.05 & N/A & 10.05 & N/A & 10.05 & N/A & 13.26 & N/A & 13.33 & N/A & 13.33 & N/A & 12.19 & N/A & 12.25 & N/A \\
\hline $\mathrm{CaO}$ & 6.41 & 6.23 & 6.41 & 6.34 & 6.41 & 6.26 & 6.41 & 6.50 & 6.41 & 7.21 & 6.41 & 7.27 & 8.55 & 8.46 & 8.57 & 8.45 \\
\hline $\mathrm{Cl}$ & 0.11 & 0.07 & 0.11 & 0.10 & 0.11 & 0.08 & 0.11 & 0.09 & 0.12 & 0.11 & 0.00 & 0.10 & 0.11 & 0.09 & 0.12 & 0.10 \\
\hline $\mathrm{Cr}_{2} \mathrm{O}_{3}$ & 0.02 & 0.02 & 0.02 & 0.02 & 0.02 & 0.02 & 0.02 & 0.02 & 0.02 & 0.02 & 0.02 & 0.02 & 0.02 & 0.02 & 0.02 & 0.02 \\
\hline $\mathrm{F}$ & 0.05 & N/A & 0.05 & N/A & 0.05 & N/A & 0.05 & N/A & 0.06 & N/A & 0.00 & N/A & 0.05 & N/A & 0.06 & N/A \\
\hline $\mathrm{Fe}_{2} \mathrm{O}_{3}$ & 6.43 & 6.40 & 6.43 & 6.54 & 6.43 & 6.64 & 0.01 & 0.09 & 0.01 & 0.08 & 0.01 & 0.06 & 0.01 & 0.08 & 0.01 & 0.04 \\
\hline $\mathrm{K}_{2} \mathrm{O}$ & 0.14 & 0.21 & 0.14 & 0.21 & 0.14 & 0.21 & 0.14 & 0.23 & 0.15 & 0.27 & 0.15 & 0.25 & 0.14 & 0.23 & 0.15 & 0.22 \\
\hline $\mathrm{Li}_{2} \mathrm{O}$ & 2.73 & N/A & 2.73 & N/A & 2.73 & N/A & 2.73 & N/A & 2.74 & N/A & 2.74 & N/A & 2.73 & N/A & 2.74 & N/A \\
\hline $\mathrm{MgO}$ & 1.50 & 1.48 & 1.50 & 1.39 & 1.50 & 1.38 & 1.50 & 1.34 & 1.51 & 1.60 & 1.51 & 1.52 & 1.50 & 1.38 & 1.51 & 1.39 \\
\hline $\mathrm{Na}_{2} \mathrm{O}$ & 11.96 & 12.13 & 11.96 & 11.73 & 11.96 & 11.24 & 11.96 & 11.58 & 11.88 & 11.16 & 11.88 & 10.62 & 11.96 & 11.60 & 11.88 & 10.20 \\
\hline $\mathrm{P}_{2} \mathrm{O}_{5}$ & 0.11 & 0.13 & 0.11 & 0.13 & 0.11 & 0.12 & 0.11 & 0.13 & 0.12 & 0.10 & 0.12 & 0.10 & 0.11 & 0.12 & 0.12 & 0.08 \\
\hline $\mathrm{Re}_{2} \mathrm{O}_{7}$ & 0.10 & 0.05 & 0.10 & 0.04 & 0.10 & 0.06 & 0.10 & 0.06 & 0.10 & 0.06 & 0.00 & 0.05 & 0.10 & 0.07 & 0.10 & 0.04 \\
\hline $\mathrm{SiO}_{2}$ & 46.74 & 47.72 & 46.74 & 48.04 & 46.74 & 47.85 & 49.95 & 50.88 & 50.00 & 57.27 & 50.00 & 57.79 & 48.88 & 49.88 & 48.92 & 50.95 \\
\hline $\mathrm{SO}_{3}$ & 0.48 & 0.29 & 0.00 & 0.01 & 2.50 & 0.66 & 0.48 & 0.35 & 2.50 & 1.07 & 2.50 & 0.94 & 0.48 & 0.41 & 2.50 & 0.84 \\
\hline $\mathrm{TiO}_{2}$ & 1.12 & 1.13 & 1.12 & 1.18 & 1.12 & 1.16 & 1.12 & 1.18 & 1.12 & 1.39 & 1.12 & 1.41 & 1.12 & 1.19 & 1.12 & 1.20 \\
\hline $\mathrm{ZnO}$ & 3.02 & 2.84 & 3.02 & 2.92 & 3.02 & 2.95 & 3.02 & 2.94 & 3.02 & 3.37 & 3.02 & 3.45 & 3.02 & 2.87 & 3.02 & 2.93 \\
\hline $\mathrm{ZrO}_{2}$ & 3.02 & 3.28 & 3.02 & 3.39 & 3.02 & 3.44 & 3.02 & 3.44 & 3.02 & 3.95 & 3.02 & 4.13 & 3.02 & 3.48 & 3.02 & 3.42 \\
\hline Sum* & 102.10 & 100.90 & 99.60 & 100.80 & 102.10 & 100.90 & 100.10 & 101.00 & 102.30 & 100.54 & 102.00 & 100.50 & 100.10 & 100.98 & 102.30 & 88.70 \\
\hline
\end{tabular}


Table 4.10. XRF Analysis of Glasses Tested for LAW AN-102 (cont'd).

\begin{tabular}{|c|c|c|c|c|c|c|c|c|c|c|c|c|c|c|c|c|}
\hline \multirow{2}{*}{$\begin{array}{c}\text { Oxide } \\
- \\
\end{array}$} & \multicolumn{2}{|c|}{ LAWC27S2W } & \multicolumn{2}{|c|}{ LAWC28 } & \multicolumn{2}{|c|}{ LAWC28S2 } & \multicolumn{2}{|c|}{ LAWC28S2W } & \multicolumn{2}{|c|}{ LAWC29 } & \multicolumn{2}{|c|}{ LAWC29S0 } & \multicolumn{2}{|c|}{ LAWC29S2 } & \multicolumn{2}{|c|}{ LAWC29S2W } \\
\hline & Target & XRF & Target & XRF & Target & XRF & Target & XRF & Target & XRF & Target & XRF & Target & XRF & Target & XRF \\
\hline $\mathrm{Al}_{2} \mathrm{O}_{3}$ & 6.13 & 6.14 & 6.12 & 6.00 & 6.13 & 5.76 & 6.13 & 5.98 & 6.55 & 6.40 & 6.55 & 6.57 & 6.55 & 6.38 & 6.55 & 6.65 \\
\hline $\mathrm{B}_{2} \mathrm{O}_{3}$ & 12.25 & N/A & 10.05 & N/A & 10.10 & N/A & 10.10 & N/A & 10.05 & N/A & 10.05 & N/A & 10.05 & N/A & 10.05 & N/A \\
\hline $\mathrm{CaO}$ & 8.57 & 8.30 & 12.82 & 12.92 & 12.89 & 12.74 & 12.89 & 12.30 & 9.62 & 9.81 & 9.62 & 9.59 & 9.62 & 9.59 & 9.62 & 9.30 \\
\hline $\mathrm{Cl}$ & 0.00 & 0.00 & 0.11 & 0.09 & 0.12 & 0.00 & 0.12 & 0.00 & 0.11 & 0.08 & 0.11 & 0.11 & 0.11 & 0.08 & 0.11 & 0.08 \\
\hline $\mathrm{Cr}_{2} \mathrm{O}_{3}$ & 0.02 & 0.02 & 0.02 & 0.02 & 0.02 & 0.02 & 0.02 & 0.02 & 0.02 & 0.02 & 0.02 & 0.02 & 0.02 & 0.02 & 0.02 & 0.02 \\
\hline $\mathrm{F}$ & 0.00 & N/A & 0.05 & N/A & 0.06 & N/A & 0.06 & N/A & 0.05 & N/A & 0.05 & N/A & 0.05 & N/A & 0.05 & N/A \\
\hline $\mathrm{Fe}_{2} \mathrm{O}_{3}$ & 0.01 & 0.04 & 0.01 & 0.07 & 0.01 & 0.05 & 0.01 & 0.03 & 0.01 & 0.10 & 0.01 & 0.10 & 0.01 & 0.11 & 0.01 & 0.09 \\
\hline $\mathrm{K}_{2} \mathrm{O}$ & 0.15 & 0.21 & 0.14 & 0.22 & 0.15 & 0.21 & 0.15 & 0.21 & 0.14 & 0.22 & 0.14 & 0.24 & 0.14 & 0.22 & 0.14 & 0.21 \\
\hline $\mathrm{Li}_{2} \mathrm{O}$ & 2.74 & N/A & 2.73 & N/A & 2.74 & N/A & 2.74 & N/A & 2.73 & N/A & 2.73 & N/A & 2.73 & N/A & 2.73 & N/A \\
\hline $\mathrm{MgO}$ & 1.51 & 1.39 & 1.50 & 1.38 & 1.51 & 1.36 & 1.51 & 1.38 & 1.50 & 1.39 & 1.50 & 1.40 & 1.50 & 1.33 & 1.50 & 1.30 \\
\hline $\mathrm{Na}_{2} \mathrm{O}$ & 11.88 & 9.84 & 11.96 & 11.38 & 11.88 & 11.18 & 11.88 & 10.76 & 11.96 & 11.31 & 11.96 & 11.85 & 11.96 & 10.75 & 11.96 & 10.77 \\
\hline $\mathrm{P}_{2} \mathrm{O}_{5}$ & 0.12 & 0.09 & 0.11 & 0.14 & 0.12 & 0.15 & 0.12 & 0.15 & 0.11 & 0.12 & 0.11 & 0.13 & 0.11 & 0.14 & 0.11 & 0.15 \\
\hline $\mathrm{Re}_{2} \mathrm{O}_{7}$ & 0.00 & 0.00 & 0.10 & 0.04 & 0.10 & 0.00 & 0.10 & 0.00 & 0.10 & 0.05 & 0.10 & 0.07 & 0.10 & 0.04 & 0.10 & 0.03 \\
\hline $\mathrm{SiO}_{2}$ & 48.92 & 51.55 & 46.74 & 47.78 & 46.77 & 47.55 & 46.77 & 48.77 & 47.18 & 48.07 & 47.18 & 48.31 & 47.18 & 48.66 & 47.18 & 49.24 \\
\hline $\mathrm{SO}_{3}$ & 2.50 & 0.76 & 0.48 & 0.43 & 2.50 & 1.50 & 2.50 & 1.10 & 0.48 & 0.37 & 0.00 & BDL & 2.50 & 1.09 & 2.50 & 0.92 \\
\hline $\mathrm{TiO}_{2}$ & 1.12 & 1.19 & 1.12 & 1.20 & 1.12 & 1.19 & 1.12 & 1.18 & 1.12 & 1.27 & 1.12 & 1.19 & 1.12 & 1.22 & 1.12 & 1.18 \\
\hline $\mathrm{ZnO}$ & 3.02 & 2.86 & 3.02 & 3.01 & 3.02 & 2.98 & 3.02 & 2.83 & 5.36 & 5.45 & 5.36 & 5.22 & 5.36 & 5.11 & 5.36 & 4.89 \\
\hline $\mathrm{ZrO}_{2}$ & 3.02 & 3.44 & 3.02 & 3.53 & 3.02 & 3.45 & 3.02 & 3.37 & 3.02 & 3.62 & 3.02 & 3.51 & 3.02 & 3.63 & 3.02 & 3.52 \\
\hline Sum* & 102.00 & 100.80 & 100.10 & 101.00 & 102.25 & 101.00 & 102.00 & 100.90 & 100.10 & 101.10 & 99.60 & 101.10 & 102.10 & 101.10 & 102.10 & 101.10 \\
\hline
\end{tabular}


Table 4.10. XRF Analysis of Glasses Tested for LAW AN-102 (cont'd).

\begin{tabular}{|c|c|c|c|c|c|c|c|c|c|c|c|c|c|c|c|c|}
\hline Oxide & \multicolumn{2}{|c|}{ LAWC30 } & \multicolumn{2}{|c|}{ LAWC30S2 } & \multicolumn{2}{|c|}{ LAWC30S2W } & \multicolumn{2}{|c|}{ LAWC31 } & \multicolumn{2}{|c|}{ LAWC31R1 } & \multicolumn{2}{|c|}{ LAWC31R2 } & \multicolumn{2}{|c|}{ LAWC31S0 } & \multicolumn{2}{|c|}{ LAWC31S2 } \\
\hline- & Target & XRF & Target & XRF & Target & XRF & Target & XRF & Target & XRF & Target & $\mathrm{XRF}$ & Target & $\mathrm{XRF}$ & Target & XRF \\
\hline $\mathrm{Al}_{2} \mathrm{O}_{3}$ & 6.12 & 6.15 & 6.12 & 6.03 & 6.12 & 6.06 & 6.12 & 6.24 & 6.12 & 5.79 & 6.12 & 6.16 & 6.12 & 6.01 & 6.12 & 6.39 \\
\hline $\mathrm{B}_{2} \mathrm{O}_{3}$ & 10.05 & N/A & 10.05 & N/A & 10.05 & N/A & 10.05 & N/A & 10.05 & N/A & 10.05 & N/A & 10.05 & N/A & 10.05 & N/A \\
\hline $\mathrm{CaO}$ & 6.41 & 6.36 & 6.41 & 6.36 & 6.41 & 6.21 & 7.41 & 7.37 & 7.41 & 7.38 & 7.41 & 7.38 & 7.41 & 7.41 & 7.41 & 7.20 \\
\hline $\mathrm{Cl}$ & 0.11 & 0.09 & 0.11 & 0.07 & 0.11 & 0.06 & 0.11 & 0.07 & 0.11 & 0.07 & 0.11 & 0.09 & 0.11 & 0.10 & 0.11 & 0.08 \\
\hline $\mathrm{Cr}_{2} \mathrm{O}_{3}$ & 0.02 & 0.02 & 0.02 & 0.02 & 0.02 & 0.02 & 0.02 & 0.02 & 0.02 & 0.03 & 0.02 & 0.02 & 0.02 & 0.02 & 0.02 & 0.02 \\
\hline $\mathrm{F}$ & 0.05 & N/A & 0.05 & N/A & 0.05 & N/A & 0.05 & N/A & 0.05 & N/A & 0.05 & N/A & 0.05 & N/A & 0.05 & N/A \\
\hline $\mathrm{Fe}_{2} \mathrm{O}_{3}$ & 4.10 & 4.30 & 4.10 & 4.33 & 4.10 & 4.34 & 4.43 & 4.64 & 4.43 & 4.81 & 4.43 & 4.63 & 4.43 & 4.75 & 4.43 & 4.63 \\
\hline $\mathrm{K}_{2} \mathrm{O}$ & 0.14 & 0.23 & 0.14 & 0.23 & 0.14 & 0.21 & 0.14 & 0.23 & 0.14 & 0.20 & 0.14 & 0.22 & 0.14 & 0.21 & 0.14 & 0.22 \\
\hline $\mathrm{Li}_{2} \mathrm{O}$ & 2.73 & N/A & 2.73 & N/A & 2.73 & N/A & 2.73 & N/A & 2.73 & N/A & 2.73 & N/A & 2.73 & N/A & 2.73 & N/A \\
\hline $\mathrm{MgO}$ & 1.50 & 1.43 & 1.50 & 1.38 & 1.50 & 1.29 & 1.50 & 1.41 & 1.50 & 1.41 & 1.50 & 1.32 & 1.50 & 1.39 & 1.50 & 1.40 \\
\hline $\mathrm{Na}_{2} \mathrm{O}$ & 11.96 & 12.23 & 11.96 & 10.86 & 11.96 & 10.70 & 11.96 & 11.53 & 11.96 & 11.87 & 11.96 & 11.57 & 11.96 & 11.98 & 11.96 & 11.05 \\
\hline $\mathrm{P}_{2} \mathrm{O}_{5}$ & 0.11 & 0.13 & 0.11 & 0.13 & 0.11 & 0.14 & 0.11 & 0.13 & 0.11 & 0.13 & 0.11 & 0.13 & 0.11 & 0.13 & 0.11 & 0.12 \\
\hline $\mathrm{Re}_{2} \mathrm{O}_{7}$ & 0.10 & 0.05 & 0.10 & 0.04 & 0.10 & 0.03 & 0.10 & 0.04 & 0.10 & 0.05 & 0.10 & 0.06 & 0.10 & 0.07 & 0.10 & 0.04 \\
\hline $\mathrm{SiO}_{2}$ & 46.74 & 47.15 & 46.74 & 47.97 & 46.74 & 48.61 & 46.74 & 47.58 & 46.74 & 46.54 & 46.74 & 47.68 & 46.74 & 47.32 & 46.74 & 47.65 \\
\hline $\mathrm{SO}_{3}$ & 0.48 & 0.34 & 2.50 & 0.90 & 2.50 & 0.70 & 0.48 & 0.39 & 0.48 & 0.34 & 0.48 & 0.39 & 0.00 & 0.01 & 2.50 & 0.86 \\
\hline $\mathrm{TiO}_{2}$ & 1.12 & 1.21 & 1.12 & 1.19 & 1.12 & 1.19 & 1.12 & 1.19 & 1.12 & 1.19 & 1.12 & 1.17 & 1.12 & 1.20 & 1.12 & 1.17 \\
\hline $\mathrm{ZnO}$ & 5.35 & 5.21 & 5.35 & 5.26 & 5.35 & 5.17 & 4.02 & 3.92 & 4.02 & 4.08 & 4.02 & 3.92 & 4.02 & 4.01 & 4.02 & 3.93 \\
\hline $\mathrm{ZrO}_{2}$ & 3.02 & 3.38 & 3.02 & 3.55 & 3.02 & 3.51 & 3.02 & 3.43 & 3.02 & 3.60 & 3.02 & 3.46 & 3.02 & 3.55 & 3.02 & 3.49 \\
\hline Sum* & 100.10 & 101.00 & 102.10 & 101.10 & 102.10 & 101.00 & 100.10 & 101.00 & 100.10 & 100.97 & 100.10 & 101.00 & 99.60 & 100.90 & 102.10 & 101.00 \\
\hline
\end{tabular}

$\mathrm{BDL}=$ Below detection limit of the instrument; N/A = Not Analyzed; Target value used for $\mathrm{B}_{2} \mathrm{O}_{3}$ and $\mathrm{Li}_{2} \mathrm{O}$; * = Includes $\mathrm{B}_{2} \mathrm{O}_{3}$ and $\mathrm{Li}_{2} \mathrm{O}$ in $\mathrm{Sum}_{\text {. }}$ 
Table 4.10. XRF Analysis of Glasses Tested for LAW AN-102 (cont'd).

\begin{tabular}{|c|c|c|c|c|c|c|c|c|c|c|c|c|}
\hline Oxide & \multicolumn{2}{|c|}{ LAWC31S2W } & \multicolumn{2}{|c|}{ LAWC32 } & \multicolumn{2}{|c|}{ LAWC32S0 } & \multicolumn{2}{|c|}{ LAWC32S2 } & \multicolumn{2}{|c|}{ LAWC32S2W } & \multicolumn{2}{|c|}{ LAWC33 } \\
\hline- & Target & XRF & Target & XRF & Target & XRF & Target & XRF & Target & XRF & Target & XRF \\
\hline $\mathrm{Al}_{2} \mathrm{O}_{3}$ & 6.12 & 6.28 & 6.49 & 6.24 & 6.49 & 6.79 & 6.49 & 6.51 & 6.49 & 6.44 & 6.14 & 6.18 \\
\hline $\mathrm{B}_{2} \mathrm{O}_{3}$ & 10.05 & N/A & 10.05 & N/A & 10.05 & N/A & 10.05 & N/A & 10.05 & N/A & 10.09 & N/A \\
\hline $\mathrm{CaO}$ & 7.41 & 7.13 & 9.04 & 9.07 & 9.04 & 8.73 & 9.04 & 8.70 & 9.04 & 8.86 & 6.94 & 6.85 \\
\hline $\mathrm{Cl}$ & 0.11 & 0.07 & 0.11 & 0.07 & 0.11 & 0.10 & 0.11 & 0.08 & 0.11 & 0.08 & 0.11 & 0.08 \\
\hline $\mathrm{Cr}_{2} \mathrm{O}_{3}$ & 0.02 & 0.02 & 0.02 & 0.02 & 0.02 & 0.02 & 0.02 & 0.02 & 0.02 & 0.02 & 0.02 & 0.02 \\
\hline $\mathrm{F}$ & 0.05 & N/A & 0.05 & N/A & 0.05 & N/A & 0.05 & N/A & 0.05 & N/A & 0.05 & N/A \\
\hline $\mathrm{Fe}_{2} \mathrm{O}_{3}$ & 4.43 & 4.57 & 2.42 & 2.62 & 2.42 & 2.49 & 2.42 & 2.53 & 2.42 & 2.62 & 4.44 & 4.58 \\
\hline $\mathrm{K}_{2} \mathrm{O}$ & 0.14 & 0.22 & 0.14 & 0.21 & 0.14 & 0.24 & 0.14 & 0.21 & 0.14 & 0.20 & 0.14 & 0.21 \\
\hline $\mathrm{Li}_{2} \mathrm{O}$ & 2.73 & N/A & 2.73 & N/A & 2.73 & N/A & 2.73 & N/A & 2.73 & N/A & 2.75 & N/A \\
\hline $\mathrm{MgO}$ & 1.50 & 1.38 & 1.50 & 1.31 & 1.50 & 1.44 & 1.50 & 1.39 & 1.50 & 1.32 & 1.51 & 1.43 \\
\hline $\mathrm{Na}_{2} \mathrm{O}$ & 11.96 & 10.50 & 11.96 & 11.55 & 11.96 & 12.00 & 11.96 & 11.34 & 11.96 & 10.58 & 12.00 & 12.77 \\
\hline $\mathrm{P}_{2} \mathrm{O}_{5}$ & 0.11 & 0.13 & 0.11 & 0.14 & 0.11 & 0.12 & 0.11 & 0.14 & 0.11 & 0.13 & 0.11 & 0.12 \\
\hline $\mathrm{Re}_{2} \mathrm{O}_{7}$ & 0.10 & 0.04 & 0.10 & 0.04 & 0.10 & 0.07 & 0.10 & 0.03 & 0.10 & 0.04 & 0.10 & 0.05 \\
\hline $\mathrm{SO}_{3}$ & 46.74 & 48.74 & 46.74 & 47.82 & 46.74 & 48.07 & 46.74 & 48.01 & 46.74 & 48.35 & 46.93 & 46.92 \\
\hline $\mathrm{SiO}_{2}$ & 2.50 & 0.77 & 0.48 & 0.38 & 0.00 & $\mathrm{BDL}$ & 2.50 & 1.09 & 2.50 & 0.95 & 0.48 & 0.37 \\
\hline $\mathrm{TiO}_{2}$ & 1.12 & 1.16 & 1.12 & 1.19 & 1.12 & 1.15 & 1.12 & 1.16 & 1.12 & 1.19 & 1.13 & 1.23 \\
\hline $\mathrm{ZnO}$ & 4.02 & 3.85 & 4.02 & 3.98 & 4.02 & 3.73 & 4.02 & 3.78 & 4.02 & 3.94 & 4.04 & 3.92 \\
\hline $\mathrm{ZrO}_{2}$ & 3.02 & 3.33 & 3.02 & 3.58 & 3.02 & 3.28 & 3.02 & 3.34 & 3.02 & 3.57 & 3.03 & 3.53 \\
\hline Sum* & 102.10 & 101.00 & 100.10 & 101.00 & 99.60 & 101.00 & 102.10 & 101.10 & 102.10 & 101.10 & 100.00 & 101.00 \\
\hline
\end{tabular}

$\mathrm{BDL}=$ Below detection limit of the instrument; N/A = Not Analyzed; Target value used for $\mathrm{B}_{2} \mathrm{O}_{3}$ and $\mathrm{Li}_{2} \mathrm{O}$; * = Includes $\mathrm{B}_{2} \mathrm{O}_{3}$ and $\mathrm{Li}_{2} \mathrm{O}$ in $\mathrm{Sum}$. 
Table 4.11. DCP Analysis of LAW Envelope A Glasses.

\begin{tabular}{|c|c|c|c|c|c|c|c|c|c|}
\hline & $\begin{array}{c}\text { LAWA44S1 } \\
\text { Target }\end{array}$ & $\begin{array}{c}\text { LAWA44S1 } \\
\text { DCP }\end{array}$ & $\begin{array}{c}\text { LAWA44SX } \\
\text { Target }\end{array}$ & $\begin{array}{c}\text { LAWA44SX } \\
\text { DCP }\end{array}$ & $\begin{array}{c}\text { LAWA88S1 } \\
\text { Target }\end{array}$ & $\begin{array}{c}\text { LAWA88S1 } \\
\text { DCP }\end{array}$ & $\begin{array}{c}\text { LAWA88S2 } \\
\text { Target }\end{array}$ & $\begin{array}{c}\text { LAWA88S2W } \\
\text { DCP }\end{array}$ & $\begin{array}{c}\text { LAWA88SX } \\
\text { DCP }\end{array}$ \\
\hline $\mathrm{Al}_{2} \mathrm{O}_{3}$ & 6.20 & 5.84 & 6.20 & 5.83 & 6.08 & 5.49 & 6.08 & 5.73 & 5.60 \\
\hline $\mathrm{B}_{2} \mathrm{O}_{3}$ & 8.90 & 8.81 & 8.90 & 8.94 & 9.70 & 9.13 & 9.70 & 9.16 & 9.42 \\
\hline $\mathrm{BaO}$ & 0.00 & 0.00 & 0.00 & 0.00 & 0.00 & 0.00 & 0.00 & 0.00 & 0.00 \\
\hline $\mathrm{CaO}$ & 1.99 & 1.99 & 1.99 & 2.07 & 1.99 & 1.87 & 1.99 & 1.88 & 1.91 \\
\hline $\mathrm{Cr}_{2} \mathrm{O}_{3}$ & 0.02 & 0.02 & 0.02 & 0.02 & 0.01 & 0.01 & 0.01 & 0.01 & 0.01 \\
\hline $\mathrm{CuO}$ & - & - & - & - & - & - & 0.00 & N/A & 0.04 \\
\hline $\mathrm{Fe}_{2} \mathrm{O}_{3}$ & 6.98 & 6.54 & 6.98 & 6.57 & 5.53 & 4.85 & 5.53 & 5.19 & 5.09 \\
\hline $\mathrm{K}_{2} \mathrm{O}$ & 0.50 & 0.49 & 0.50 & 0.43 & 2.58 & 2.16 & 2.58 & 2.08 & 1.92 \\
\hline $\mathrm{Li}_{2} \mathrm{O}$ & 0.00 & 0.03 & 0.00 & 0.04 & 0.00 & 0.03 & 0.00 & 0.04 & N/A \\
\hline $\mathrm{MgO}$ & 1.99 & 1.70 & 1.99 & 1.72 & 1.48 & 1.44 & 1.48 & 1.40 & 1.45 \\
\hline $\mathrm{Na}_{2} \mathrm{O}$ & 20.00 & 16.93 & 21.35 & 17.76 & 20.00 & 17.74 & 20.00 & 16.49 & 17.92 \\
\hline $\mathrm{SiO}_{2}$ & 44.55 & 41.16 & 44.55 & 43.29 & 43.99 & 43.31 & 43.99 & 43.23 & 41.77 \\
\hline $\mathrm{SrO}$ & 0.00 & 0.00 & 0.00 & N/A & 0.00 & N/A & 0.00 & 0.01 & 0.00 \\
\hline $\mathrm{TiO}_{2}$ & 1.99 & 1.91 & 1.99 & 1.92 & 1.99 & 1.81 & 1.99 & 1.95 & 1.85 \\
\hline $\mathrm{ZnO}$ & 2.96 & 2.90 & 2.96 & 2.90 & 2.95 & 2.85 & 2.95 & 2.77 & 2.78 \\
\hline $\mathrm{ZrO}_{2}$ & 2.99 & 3.16 & 2.99 & 3.03 & 2.99 & 2.81 & 2.99 & 3.03 & 2.98 \\
\hline $\mathrm{Cl}$ & 0.65 & N/A & 0.65 & N/A & 0.33 & N/A & 0.33 & N/A & N/A \\
\hline $\mathrm{F}$ & - & - & - & - & 0.00 & N/A & - & - & - \\
\hline $\mathrm{P}_{2} \mathrm{O}_{5}$ & 0.03 & 0.20 & 0.03 & 0.13 & 0.07 & 0.22 & 0.07 & 0.17 & 0.17 \\
\hline $\mathrm{SO}_{3}$ & 1.48 & 0.60 & 2.50 & 0.46 & 1.40 & 0.60 & 2.50 & 0.46 & 0.55 \\
\hline $\mathrm{Re}_{2} \mathrm{O}_{7}$ & 0.10 & N/A & 0.10 & N/A & 0.10 & N/A & 0.10 & N/A & N/A \\
\hline SUM & 101.38 & 92.28 & 103.75 & 95.11 & 101.19 & 94.32 & 102.29 & 93.60 & 93.46 \\
\hline
\end{tabular}

N/A = Not Analyzed 
Table 4.11. DCP Analysis of LAW Envelope A Glasses (cont'd).

\begin{tabular}{|c|c|c|c|c|c|c|c|}
\hline & $\begin{array}{c}\text { LAWA108S2 } \\
\text { Target }\end{array}$ & $\begin{array}{c}\text { LAWA108S2 } \\
\text { DCP }\end{array}$ & $\begin{array}{c}\text { LAWA109S1 } \\
\text { Target }\end{array}$ & $\begin{array}{c}\text { LAWA109S1 } \\
\text { DCP }\end{array}$ & $\begin{array}{c}\text { LAWA109S1W } \\
\text { DCP }\end{array}$ & $\begin{array}{c}\text { LAWA109S2 } \\
\text { Target }\end{array}$ & $\begin{array}{c}\text { LAWA109S2W } \\
\text { DCP }\end{array}$ \\
\hline $\mathrm{Al}_{2} \mathrm{O}_{3}$ & 6.20 & 6.05 & 6.20 & 5.84 & 5.92 & 6.20 & 5.86 \\
\hline $\mathrm{B}_{2} \mathrm{O}_{3}$ & 12.39 & 12.57 & 10.65 & 10.66 & 10.45 & 10.65 & 10.47 \\
\hline $\mathrm{BaO}$ & - & 0.03 & - & 0.00 & 0.00 & - & 0.00 \\
\hline $\mathrm{CaO}$ & 1.99 & 1.98 & 1.99 & 1.98 & 1.96 & 1.99 & 1.91 \\
\hline $\mathrm{Cr}_{2} \mathrm{O}_{3}$ & 0.02 & 0.02 & 0.02 & 0.02 & 0.02 & 0.02 & 0.02 \\
\hline $\mathrm{CuO}$ & - & 0.06 & 3.49 & 3.33 & 3.36 & 3.49 & 3.37 \\
\hline $\mathrm{Fe}_{2} \mathrm{O}_{3}$ & 0.50 & 0.46 & 0.50 & 0.53 & 0.49 & 0.50 & 0.41 \\
\hline $\mathrm{K}_{2} \mathrm{O}$ & - & 0.03 & - & 0.03 & 0.04 & - & 0.02 \\
\hline $\mathrm{Li}_{2} \mathrm{O}$ & 1.99 & 1.74 & 1.99 & 1.73 & 1.70 & 1.99 & 1.67 \\
\hline $\mathrm{MgO}$ & 0.01 & N/A & 0.01 & N/A & N/A & 0.01 & N/A \\
\hline $\mathrm{Na}_{2} \mathrm{O}$ & 20.00 & 17.28 & 20.00 & 17.75 & 17.60 & 20.00 & 16.58 \\
\hline $\mathrm{SiO}_{2}$ & 48.04 & 47.11 & 46.30 & 44.81 & 45.50 & 46.30 & 43.89 \\
\hline $\mathrm{SrO}$ & - & 0.01 & - & 0.00 & 0.00 & - & 0.00 \\
\hline $\mathrm{TiO}_{2}$ & 1.99 & 1.96 & 1.99 & 1.85 & 1.87 & 1.99 & 1.88 \\
\hline $\mathrm{ZnO}$ & 2.97 & 2.92 & 2.97 & 2.85 & 2.82 & 2.97 & 2.79 \\
\hline $\mathrm{ZrO}_{2}$ & 2.99 & 3.09 & 2.99 & 3.01 & 3.04 & 2.99 & 3.09 \\
\hline $\mathrm{Cl}$ & 0.65 & N/A & 0.65 & N/A & N/A & 0.65 & N/A \\
\hline $\mathrm{F}$ & 0.01 & N/A & 0.01 & N/A & N/A & 0.01 & N/A \\
\hline $\mathrm{P}_{2} \mathrm{O}_{5}$ & 0.03 & 0.18 & 0.03 & 0.21 & 0.21 & 0.03 & 0.14 \\
\hline $\mathrm{SO}_{3}$ & 2.50 & 0.58 & 1.48 & 0.49 & 0.47 & 2.50 & 0.65 \\
\hline $\mathrm{Re}_{2} \mathrm{O}_{7}$ & 0.10 & N/A & 0.10 & $\mathrm{~N} / \mathrm{A}$ & N/A & 0.10 & N/A \\
\hline SUM & 102.40 & 96.07 & 101.38 & 95.09 & 95.45 & 102.40 & 92.75 \\
\hline
\end{tabular}


Table 4.11. DCP Analysis of LAW Envelope A Glasses (cont'd).

\begin{tabular}{|c|c|c|c|c|c|c|c|c|c|c|}
\hline & $\begin{array}{c}\text { LAWA110S1 } \\
\text { Target }\end{array}$ & $\begin{array}{c}\text { LAWA110S1 } \\
\text { DCP }\end{array}$ & $\begin{array}{c}\text { LAWA110S1W } \\
\text { DCP }\end{array}$ & $\begin{array}{c}\text { LAWA110S2 } \\
\text { Target }\end{array}$ & $\begin{array}{c}\text { LAWA110S2W } \\
\text { DCP }\end{array}$ & $\begin{array}{c}\text { LAWA111S1 } \\
\text { Target }\end{array}$ & $\begin{array}{c}\text { LAWA111S1 } \\
\text { DCP }\end{array}$ & $\begin{array}{c}\text { LAWA111S2 } \\
\text { Target }\end{array}$ & $\begin{array}{c}\text { LAWA111S2 } \\
\text { DCP }\end{array}$ & $\begin{array}{c}\text { LAWA111S2W } \\
\text { DCP }\end{array}$ \\
\hline $\mathrm{Al}_{2} \mathrm{O}_{3}$ & 7.36 & 6.85 & 7.06 & 7.36 & 6.74 & 6.20 & 6.08 & 6.20 & 6.14 & 5.75 \\
\hline $\mathrm{B}_{2} \mathrm{O}_{3}$ & 10.07 & 9.99 & 10.09 & 10.07 & 9.56 & 8.90 & 9.66 & 8.90 & 10.05 & 9.02 \\
\hline $\mathrm{BaO}$ & 0.00 & 0.00 & 0.00 & 0.00 & 0.00 & 0.00 & 0.00 & 0.00 & 0.00 & 0.00 \\
\hline $\mathrm{CaO}$ & 3.16 & 2.99 & 3.04 & 3.16 & 2.91 & 8.98 & 8.29 & 8.98 & 8.29 & 8.33 \\
\hline $\mathrm{Cr}_{2} \mathrm{O}_{3}$ & 0.02 & 0.02 & 0.02 & 0.02 & 0.02 & 0.02 & 0.02 & 0.02 & 0.02 & 0.02 \\
\hline $\mathrm{Fe}_{2} \mathrm{O}_{3}$ & 3.49 & 3.32 & 3.43 & 3.49 & 3.23 & 0.00 & 0.05 & 0.00 & 0.06 & 0.05 \\
\hline $\mathrm{K}_{2} \mathrm{O}$ & 0.50 & 0.47 & 0.48 & 0.50 & 0.47 & 0.50 & 0.56 & 0.50 & 0.49 & 0.50 \\
\hline $\mathrm{Li}_{2} \mathrm{O}$ & 0.00 & 0.03 & 0.04 & 0.00 & 0.04 & 0.00 & 0.09 & 0.00 & 0.09 & 0.06 \\
\hline $\mathrm{MgO}$ & 1.99 & 1.70 & 1.89 & 1.99 & 1.63 & 1.99 & 1.84 & 1.99 & 1.86 & 1.81 \\
\hline $\mathrm{MoO}_{3}$ & 0.01 & N/A & N/A & 0.01 & N/A & - & - & - & - & - \\
\hline $\mathrm{Na}_{2} \mathrm{O}$ & 20.00 & 17.56 & 17.76 & 20.00 & 16.30 & 20.00 & 16.92 & 20.00 & 16.24 & 15.54 \\
\hline $\mathrm{SiO}_{2}$ & 44.55 & 43.37 & 44.17 & 44.55 & 42.70 & 44.55 & 43.96 & 44.55 & 44.21 & 43.32 \\
\hline $\mathrm{SrO}$ & 0.00 & 0.00 & 0.00 & 0.00 & 0.00 & 0.00 & 0.01 & 0.00 & 0.01 & 0.00 \\
\hline $\mathrm{TiO}_{2}$ & 1.99 & 1.83 & 1.94 & 1.99 & 1.87 & 1.99 & 1.94 & 1.99 & 2.04 & 1.93 \\
\hline $\mathrm{ZnO}$ & 2.96 & 2.83 & 2.86 & 2.96 & 2.72 & 2.96 & 2.96 & 2.96 & 2.92 & 2.86 \\
\hline $\mathrm{ZrO}_{2}$ & 2.99 & 2.99 & 3.07 & 2.99 & 2.95 & 2.99 & 3.12 & 2.99 & 3.20 & 2.98 \\
\hline $\mathrm{Cl}$ & 0.65 & N/A & N/A & 0.65 & N/A & 0.65 & N/A & 0.65 & N/A & N/A \\
\hline $\mathrm{F}$ & 0.01 & N/A & N/A & 0.01 & N/A & - & - & - & - & - \\
\hline $\mathrm{P}_{2} \mathrm{O}_{5}$ & 0.03 & 0.14 & 0.25 & 0.03 & 0.14 & 0.03 & 0.19 & 0.03 & 0.24 & 0.21 \\
\hline $\mathrm{SO}_{3}$ & 1.48 & 0.65 & 0.35 & 2.50 & 0.40 & 1.48 & 0.97 & 2.50 & 0.82 & 0.35 \\
\hline $\mathrm{Re}_{2} \mathrm{O}_{7}$ & 0.10 & N/A & N/A & 0.10 & N/A & 0.10 & N/A & 0.10 & N/A & N/A \\
\hline SUM & 101.38 & 94.74 & 96.45 & 102.40 & 91.68 & 101.38 & 96.66 & 102.40 & 96.68 & 92.73 \\
\hline
\end{tabular}

N/A = Not Analyzed 
Table 4.11. DCP Analysis of LAW Envelope A Glasses (cont'd).

\begin{tabular}{|c|c|c|c|c|c|c|c|c|c|c|}
\hline & $\begin{array}{c}\text { LAWA112S1 } \\
\text { Target }\end{array}$ & $\begin{array}{c}\text { LAWA112S1 } \\
\text { DCP }\end{array}$ & $\begin{array}{c}\text { LAWA112S2 } \\
\text { Target }\end{array}$ & $\begin{array}{c}\text { LAWA112S2 } \\
\text { DCP }\end{array}$ & $\begin{array}{c}\text { LAWA112S2W } \\
\text { DCP }\end{array}$ & $\begin{array}{c}\text { LAWA118S2 } \\
\text { Target }\end{array}$ & $\begin{array}{c}\text { LAWA118S2 } \\
\text { DCP }\end{array}$ & $\begin{array}{c}\text { LAWA119S1 } \\
\text { Target }\end{array}$ & $\begin{array}{c}\text { LAWA119S1 } \\
\text { DCP }\end{array}$ & $\begin{array}{c}\text { LAWA119S1W } \\
\text { DCP }\end{array}$ \\
\hline $\mathrm{Al}_{2} \mathrm{O}_{3}$ & 6.08 & 5.79 & 6.08 & 5.74 & 5.78 & 6.06 & 5.40 & 6.06 & 5.80 & 5.45 \\
\hline $\mathrm{B}_{2} \mathrm{O}_{3}$ & 9.70 & 10.14 & 9.70 & 9.74 & 10.03 & 10.00 & 10.30 & 10.00 & 10.09 & 10.33 \\
\hline $\mathrm{BaO}$ & 0.00 & 0.00 & 0.00 & 0.00 & 0.00 & 0.00 & 0.00 & 0.00 & 0.00 & 0.00 \\
\hline $\mathrm{CaO}$ & 7.52 & 7.28 & 7.52 & 7.13 & 7.23 & 5.07 & 4.60 & 10.48 & 9.26 & 9.30 \\
\hline $\mathrm{Cr}_{2} \mathrm{O}_{3}$ & 0.01 & 0.02 & 0.01 & 0.01 & 0.02 & 0.02 & 0.31 & 0.02 & 0.02 & 0.03 \\
\hline $\mathrm{Fe}_{2} \mathrm{O}_{3}$ & 0.00 & 0.05 & - & - & - & - & - & - & - & - \\
\hline $\mathrm{K}_{2} \mathrm{O}$ & 2.58 & 2.31 & 0.00 & 0.04 & 0.04 & 5.41 & 6.16 & 0.00 & 0.05 & 0.06 \\
\hline $\mathrm{Li}_{2} \mathrm{O}$ & 0.00 & 0.08 & 2.58 & 2.20 & 2.15 & 2.76 & 2.37 & 2.76 & 2.44 & 2.44 \\
\hline $\mathrm{MgO}$ & 1.48 & 1.69 & 0.00 & 0.05 & 0.07 & 0.00 & 0.06 & 0.00 & 0.06 & 0.08 \\
\hline $\mathrm{MoO}_{3}$ & - & - & 1.48 & 1.49 & 1.55 & 1.50 & 1.63 & 1.50 & 1.41 & 1.60 \\
\hline $\mathrm{Na}_{2} \mathrm{O}$ & 20.00 & 17.10 & 20.00 & 16.39 & 16.30 & 14.49 & 11.78 & 14.49 & 11.85 & 11.99 \\
\hline $\mathrm{SiO}_{2}$ & 43.99 & 43.06 & 43.99 & 42.28 & 43.23 & 46.60 & 44.65 & 46.60 & 44.51 & 45.07 \\
\hline $\mathrm{SrO}$ & 0.00 & 0.01 & 0.00 & N/A & N/A & 0.00 & 0.01 & 0.00 & 0.01 & 0.01 \\
\hline $\mathrm{TiO}_{2}$ & 1.99 & 1.95 & 1.99 & 1.96 & 2.02 & 1.14 & 1.39 & 1.14 & 1.20 & 1.34 \\
\hline $\mathrm{ZnO}$ & 2.95 & 2.87 & 2.95 & 2.93 & 2.85 & 3.06 & 2.91 & 3.06 & 2.86 & 3.02 \\
\hline $\mathrm{ZrO}_{2}$ & 2.99 & 3.05 & 2.99 & 3.11 & 3.10 & 3.02 & 2.82 & 3.02 & 2.96 & 2.77 \\
\hline $\mathrm{Cl}$ & 0.33 & N/A & 0.33 & N/A & N/A & 0.33 & N/A & 0.33 & N/A & N/A \\
\hline $\mathrm{F}$ & - & - & - & - & - & - & - & - & - & - \\
\hline $\mathrm{P}_{2} \mathrm{O}_{5}$ & 0.07 & 0.21 & 0.07 & 0.17 & 0.27 & 0.13 & 0.32 & 0.13 & 0.25 & 0.36 \\
\hline $\mathrm{SO}_{3}$ & 1.50 & 0.80 & 2.50 & 0.85 & 0.85 & 2.50 & 0.54 & 1.48 & 0.51 & 0.60 \\
\hline $\mathrm{Re}_{2} \mathrm{O}_{7}$ & 0.10 & N/A & 0.10 & N/A & N/A & 0.10 & N/A & 0.10 & N/A & N/A \\
\hline SUM & 101.29 & 96.41 & 102.29 & 94.09 & 95.49 & 102.23 & 95.25 & 101.21 & 93.28 & 94.45 \\
\hline
\end{tabular}


Table 4.11. DCP Analysis of LAW Envelope A Glasses (cont'd).

\begin{tabular}{|c|c|c|c|c|c|c|c|}
\hline & $\begin{array}{c}\text { LAWA120S1 } \\
\text { Target } \\
\end{array}$ & $\begin{array}{c}\text { LAWA120S1 } \\
\text { DCP } \\
\end{array}$ & $\begin{array}{c}\text { LAWA120S2 } \\
\text { Target } \\
\end{array}$ & $\begin{array}{c}\text { LAWA120S2 } \\
\text { DCP } \\
\end{array}$ & $\begin{array}{c}\text { LAWA120S2 } \\
\text { DCP }\end{array}$ & $\begin{array}{c}\text { LAWA121S1 } \\
\text { Target }\end{array}$ & $\begin{array}{c}\text { LAWA121S2W } \\
\text { DCP }\end{array}$ \\
\hline $\mathrm{Al}_{2} \mathrm{O}_{3}$ & 5.82 & 5.35 & 5.82 & 5.44 & 5.3 & 5.82 & 5.42 \\
\hline $\mathrm{B}_{2} \mathrm{O}_{3}$ & 9.3 & 9.66 & 9.3 & 9.77 & 9.73 & 9.3 & 9.39 \\
\hline $\mathrm{BaO}$ & 0 & 0 & 0 & 0 & 0 & 0 & 0 \\
\hline $\mathrm{CaO}$ & 4.71 & 4.48 & 4.71 & 4.56 & 4.51 & 9.74 & 8.45 \\
\hline $\mathrm{Cr}_{2} \mathrm{O}_{3}$ & 0.02 & 0.02 & 0.02 & 0.02 & 0.02 & 0.02 & 0.02 \\
\hline $\mathrm{CuO}$ & - & - & - & - & - & - & - \\
\hline $\mathrm{Fe}_{2} \mathrm{O}_{3}$ & 5.03 & 4.91 & 5.03 & 4.98 & 4.91 & 0 & 0.04 \\
\hline $\mathrm{K}_{2} \mathrm{O}$ & 8.31 & 7.24 & 8.31 & 7.11 & 6.95 & 8.31 & 6.64 \\
\hline $\mathrm{Li}_{2} \mathrm{O}$ & 0 & 0.06 & 0 & 0.05 & 0.06 & 0 & 0.06 \\
\hline $\mathrm{MgO}$ & 1.39 & 1.52 & 1.39 & 1.6 & 1.42 & 1.39 & 1.4 \\
\hline $\mathrm{Na}_{2} \mathrm{O}$ & 14.49 & 12.23 & 14.49 & 11.91 & 12.07 & 14.49 & 10.51 \\
\hline $\mathrm{SiO}_{2}$ & 43.34 & 40.66 & 43.34 & 42.98 & 43.51 & 43.34 & 40.14 \\
\hline $\mathrm{SrO}$ & 0 & 0.01 & 0 & 0 & 0 & 0 & 0.01 \\
\hline $\mathrm{TiO}_{2}$ & 1.06 & 1.25 & 1.06 & 1.29 & 1.19 & 1.06 & 1.14 \\
\hline $\mathrm{ZnO}$ & 2.85 & 2.71 & 2.85 & 2.89 & 2.83 & 2.85 & 2.91 \\
\hline $\mathrm{ZrO}_{2}$ & 2.81 & 2.68 & 2.81 & 2.79 & 2.74 & 2.81 & 2.8 \\
\hline $\mathrm{Cl}$ & 0.33 & N/A & 0.33 & N/A & N/A & 0.33 & N/A \\
\hline $\mathrm{F}$ & - & - & - & - & - & - & - \\
\hline $\mathrm{P}_{2} \mathrm{O}_{5}$ & 0.13 & 0.35 & 0.13 & 0.26 & 0.32 & 0.13 & 0.27 \\
\hline $\mathrm{SO}_{3}$ & 1.48 & 0.44 & 2.5 & 0.54 & 0.48 & 1.48 & 0.43 \\
\hline $\mathrm{Re}_{2} \mathrm{O}_{7}$ & 0.1 & N/A & 0.1 & N/A & N/A & 0.1 & N/A \\
\hline SUM & 101.21 & 93.57 & 102.23 & 96.19 & 96.04 & 101.21 & 89.63 \\
\hline
\end{tabular}

N/A = Not Analyzed 
Table 4.11. DCP Analysis of LAW Envelope A Glasses (cont'd).

\begin{tabular}{|c|c|c|c|c|c|c|c|}
\hline & $\begin{array}{c}\text { LAWA122S1 } \\
\text { Target }\end{array}$ & $\begin{array}{c}\text { LAWA122S1 } \\
\text { DCP }\end{array}$ & $\begin{array}{c}\text { LAWA122S2 } \\
\text { Target } \\
\end{array}$ & $\begin{array}{c}\text { LAWA122S2 } \\
\text { DCP }\end{array}$ & $\begin{array}{c}\text { LAWA122S2W } \\
\text { DCP }\end{array}$ & $\begin{array}{c}\text { LAWA123S2 } \\
\text { Target }\end{array}$ & $\begin{array}{c}\text { LAWA123S2 } \\
\text { DCP } \\
\end{array}$ \\
\hline $\mathrm{Al}_{2} \mathrm{O}_{3}$ & 5.93 & 5.38 & 5.93 & 5.58 & 5.56 & 5.93 & 5.52 \\
\hline $\mathrm{B}_{2} \mathrm{O}_{3}$ & 9.64 & 9.56 & 9.64 & 9.77 & 9.93 & 9.64 & 9.89 \\
\hline $\mathrm{BaO}$ & 0 & 0 & 0 & 0 & 0 & 0 & 0 \\
\hline $\mathrm{CaO}$ & 4.88 & 4.65 & 4.88 & 4.58 & 4.48 & 10.1 & 9.09 \\
\hline $\mathrm{Cr}_{2} \mathrm{O}_{3}$ & 0.02 & 0.02 & 0.02 & 0.02 & 0.02 & 0.02 & 0.02 \\
\hline $\mathrm{CuO}$ & - & - & - & - & - & - & - \\
\hline $\mathrm{Fe}_{2} \mathrm{O}_{3}$ & 5.21 & 4.94 & 5.21 & 4.99 & 5.19 & 0 & 0.04 \\
\hline $\mathrm{K}_{2} \mathrm{O}$ & 5.64 & 4.84 & 5.64 & 4.6 & 4.5 & 5.64 & 4.55 \\
\hline $\mathrm{Li}_{2} \mathrm{O}$ & 0 & 0.04 & 0 & 0.04 & 0.04 & 0 & 0.06 \\
\hline $\mathrm{MgO}$ & 1.44 & 1.49 & 1.44 & 1.49 & 1.38 & 1.44 & 1.41 \\
\hline $\mathrm{Na}_{2} \mathrm{O}$ & 14.49 & 12.25 & 14.49 & 11.86 & 10.5 & 14.49 & 11.57 \\
\hline $\mathrm{SiO}_{2}$ & 44.91 & 44.11 & 44.91 & 43.4 & 42.61 & 44.91 & 43.91 \\
\hline $\mathrm{SrO}$ & 0 & 0 & 0 & 0 & 0 & 0 & 0.01 \\
\hline $\mathrm{TiO}_{2}$ & 1.1 & 1.26 & 1.1 & 1.26 & 1.19 & 1.1 & 1.16 \\
\hline $\mathrm{ZnO}$ & 2.95 & 2.87 & 2.95 & 2.97 & 3.01 & 2.95 & 2.95 \\
\hline $\mathrm{ZrO}_{2}$ & 2.91 & 2.88 & 2.91 & 2.91 & 3.02 & 2.91 & 2.86 \\
\hline $\mathrm{Cl}$ & 0.33 & N/A & 0.33 & N/A & N/A & 0.33 & N/A \\
\hline $\mathrm{F}$ & - & - & - & - & - & - & - \\
\hline $\mathrm{P}_{2} \mathrm{O}_{5}$ & 0.13 & 0.2 & 0.13 & 0.2 & 0.26 & 0.13 & 0.33 \\
\hline $\mathrm{SO}_{3}$ & 1.48 & 0.47 & 2.5 & 0.47 & 0.47 & 2.5 & 1.04 \\
\hline $\mathrm{Re}_{2} \mathrm{O}_{7}$ & 0.1 & N/A & 0.1 & N/A & N/A & 0.1 & N/A \\
\hline SUM & 101.21 & 94.96 & 102.23 & 94.14 & 92.16 & 102.23 & 94.41 \\
\hline
\end{tabular}

N/A = Not Analyzed 
The Catholic University of America

Vitreous State Laboratory
ORP-55237, Rev. 0

Baseline LAW Glass Formulation Testing Final Report, VSL-03R3460-1, Rev. 0

Table 4.12. DCP Analysis of LAW Envelope B Glasses.

\begin{tabular}{|c|c|c|c|c|c|c|c|c|c|c|}
\hline Oxide & \multicolumn{2}{|c|}{ LAWB60S2 } & \multicolumn{3}{|c|}{ LAWB60S4 } & \multicolumn{3}{|c|}{ LAWB60S4W } & \multicolumn{2}{|c|}{ LAWB72 } \\
\hline- & Target & DCP & Target & DCP & DCP & Target & DCP & DCP & Target & DCP \\
\hline $\mathrm{Al}_{2} \mathrm{O}_{3}$ & 6.129 & 5.71 & 6.129 & 5.67 & 5.86 & 6.129 & 5.57 & 5.16 & 6.151 & 5.43 \\
\hline $\mathrm{B}_{2} \mathrm{O}_{3}$ & 12.34 & 12.4 & 12.34 & 12.15 & 12.39 & 12.34 & 12.87 & 12.36 & 12.33 & 11.88 \\
\hline $\mathrm{CaO}$ & 11.882 & 10.27 & 11.882 & 10.41 & 10.67 & 11.882 & 9.81 & 9.66 & 7.124 & 6.39 \\
\hline $\mathrm{Cl}$ & 0.006 & N/A & 0.006 & N/A & N/A & 0.006 & N/A & N/A & 0.008 & N/A \\
\hline $\mathrm{Cr}_{2} \mathrm{O}_{3}$ & 0.073 & 0.07 & 0.073 & 0.07 & 0.06 & 0.073 & 0.07 & 0.07 & 0.047 & 0.24 \\
\hline $\mathrm{Cs}_{2} \mathrm{O}$ & 0.002 & N/A & 0.002 & N/A & N/A & 0.002 & N/A & N/A & 0 & N/A \\
\hline $\mathrm{F}$ & 0.081 & N/A & 0.081 & N/A & N/A & 0.081 & N/A & N/A & 0.078 & N/A \\
\hline $\mathrm{Fe}_{2} \mathrm{O}_{3}$ & 0 & 0.04 & 0 & 0.05 & 0.05 & 0 & 0.04 & 0.04 & 3.252 & 3.68 \\
\hline $\mathrm{K}_{2} \mathrm{O}$ & 0.262 & 0.31 & 0.262 & 0.3 & 0.23 & 0.262 & 0.25 & 0.23 & 0.234 & 0.23 \\
\hline $\mathrm{Li}_{2} \mathrm{O}$ & 4.616 & 4.14 & 4.616 & 3.83 & 4.2 & 4.616 & 3.76 & 3.51 & 4.11 & 3.76 \\
\hline $\mathrm{MgO}$ & 2.971 & 2.51 & 2.971 & 2.54 & 2.68 & 2.971 & 1.4 & 2.7 & 2.968 & 2.51 \\
\hline $\mathrm{Na}_{2} \mathrm{O}$ & 6.5 & 5.74 & 6.5 & 5.01 & 5.12 & 6.5 & 4.82 & 4.51 & 6.62 & 5.98 \\
\hline $\mathrm{P}_{2} \mathrm{O}_{5}$ & 0.03 & 0.19 & 0.03 & 0.1 & 0.19 & 0.03 & 0.13 & 0.19 & 0.045 & 0.14 \\
\hline $\mathrm{Re}_{2} \mathrm{O}_{7}$ & 0.1 & N/A & 0.1 & N/A & N/A & 0.1 & N/A & N/A & 0.1 & N/A \\
\hline $\mathrm{SiO}_{2}$ & 47.859 & 45.02 & 47.859 & 45.73 & 46.99 & 47.859 & 45.88 & 44.84 & 47.949 & 44.92 \\
\hline $\mathrm{SO}_{3}$ & 2.5 & 1.38 & 4.5 & 1.33 & 1.4 & 4.5 & 1.57 & 1.45 & 0.782 & N/A \\
\hline $\mathrm{TiO}_{2}$ & 0 & 0.02 & 0 & 0.03 & 0.03 & 0 & 0.03 & 0.03 & 0 & 0.03 \\
\hline $\mathrm{ZnO}$ & 3.153 & 3.07 & 3.153 & 3.17 & 3.14 & 3.153 & 3.07 & 2.97 & 5.151 & 4.96 \\
\hline $\mathrm{ZrO}_{2}$ & 3.153 & 3 & 3.153 & 3.04 & 3.12 & 3.153 & 2.95 & 2.83 & 3.151 & 2.94 \\
\hline Sum & 101.7 & 93.87 & 103.7 & 93.43 & 96.13 & 103.7 & 92.22 & 90.55 & 100.1 & 93.09 \\
\hline
\end{tabular}


The Catholic University of America

Vitreous State Laboratory
ORP-55237, Rev. 0

Baseline LAW Glass Formulation Testing

Final Report, VSL-03R3460-1, Rev. 0

Table 4.12. DCP Analysis for LAW Envelope B Glasses (cont'd).

\begin{tabular}{|c|c|c|c|c|c|c|c|c|c|c|}
\hline Oxide & \multicolumn{2}{|c|}{ LAWB77 } & \multicolumn{2}{|c|}{ LAWB81 } & \multicolumn{2}{|c|}{ LAWB82 } & \multicolumn{2}{|c|}{ LAWB83 } & \multicolumn{2}{|c|}{ LAWB83S4W } \\
\hline- & Target & DCP & Target & DCP & Target & DCP & Target & DCP & Target & $\mathrm{DCP}$ \\
\hline $\mathrm{Al}_{2} \mathrm{O}_{3}$ & 6.151 & 5.35 & 6.151 & 5.23 & 6.151 & 5.09 & 6.183 & 5.48 & 6.183 & 5.83 \\
\hline $\mathrm{B}_{2} \mathrm{O}_{3}$ & 12.33 & 11.84 & 12.33 & 11.85 & 10.083 & 9.66 & 10.026 & 9.78 & 10.026 & 9.89 \\
\hline $\mathrm{CaO}$ & 6.621 & 5.85 & 7.124 & 6.34 & 7.124 & 6.26 & 6.777 & 5.96 & 6.777 & 5.83 \\
\hline $\mathrm{Cl}$ & 0.008 & $\mathrm{~N} / \mathrm{A}$ & 0.008 & N/A & 0.008 & N/A & N/A & N/A & 0.007 & N/A \\
\hline $\mathrm{Cr}_{2} \mathrm{O}_{3}$ & 0.047 & 0.2 & 0.047 & 0.25 & 0.047 & 0.26 & 0.039 & 0.04 & 0.039 & 0.03 \\
\hline $\mathrm{Cs}_{2} \mathrm{O}$ & 0 & $\mathrm{~N} / \mathrm{A}$ & 0 & $\mathrm{~N} / \mathrm{A}$ & 0 & N/A & N/A & N/A & 0 & N/A \\
\hline $\mathrm{F}$ & 0.078 & $\mathrm{~N} / \mathrm{A}$ & 0.078 & N/A & 0.078 & N/A & N/A & N/A & 0.064 & N/A \\
\hline $\mathrm{Fe}_{2} \mathrm{O}_{3}$ & 2.202 & 2.62 & 3.252 & 3.73 & 9.499 & 9.42 & 5.292 & 4.93 & 5.292 & 5.13 \\
\hline $\mathrm{K}_{2} \mathrm{O}$ & 0.234 & 0.23 & 0.234 & 0.23 & 0.234 & 0.22 & 0.194 & 0.18 & 0.194 & 0.18 \\
\hline $\mathrm{Li}_{2} \mathrm{O}$ & 4.11 & 3.73 & 4.261 & 3.88 & 4.261 & 3.91 & 4.307 & 3.81 & 4.307 & 3.55 \\
\hline $\mathrm{MgO}$ & 2.968 & 2.5 & 2.968 & 2.62 & 1.48 & 1.46 & 2.971 & 2.63 & 2.971 & 2.71 \\
\hline $\mathrm{Na}_{2} \mathrm{O}$ & 6.62 & 5.95 & 6.62 & 5.88 & 6.62 & 5.91 & 5.472 & 4.89 & 5.472 & 4.37 \\
\hline $\mathrm{P}_{2} \mathrm{O}_{5}$ & 0.045 & 0.14 & 0.045 & 0.1 & 0.045 & 0.19 & 0.037 & 0.17 & 0.037 & 0.18 \\
\hline $\mathrm{Re}_{2} \mathrm{O}_{7}$ & 0.1 & N/A & 0.1 & N/A & 0.1 & N/A & N/A & N/A & 0.1 & N/A \\
\hline $\mathrm{SiO}_{2}$ & 47.949 & 43.42 & 47.949 & 43.98 & 45.437 & 42.17 & 48.599 & 45.6 & 48.599 & 46.55 \\
\hline $\mathrm{SO}_{3}$ & 0.782 & N/A & 0.782 & N/A & 0.782 & N/A & 0.646 & 0.6 & 4.5 & 1.21 \\
\hline $\mathrm{TiO}_{2}$ & 1.553 & 1.47 & 0 & 0.02 & 0 & 0.02 & 1.393 & 1.39 & 1.393 & 1.41 \\
\hline $\mathrm{ZnO}$ & 5.151 & 4.85 & 5 & 4.68 & 5 & 4.74 & 4.837 & 4.45 & 4.837 & 4.45 \\
\hline $\mathrm{ZrO}_{2}$ & 3.151 & 2.96 & 3.151 & 2.99 & 3.151 & 2.97 & 3.156 & 2.97 & 3.156 & 3.1 \\
\hline Sum & 100.1 & 91.11 & 100.1 & 91.78 & 100.1 & 92.28 & 99.928 & 92.87 & 104 & 94.43 \\
\hline
\end{tabular}


The Catholic University of America

Vitreous State Laboratory
ORP-55237, Rev. 0

Baseline LAW Glass Formulation Testing

Final Report, VSL-03R3460-1, Rev. 0

Table 4.13. DCP Analysis of LAW Envelope C Glasses.

\begin{tabular}{|c|c|c|c|c|c|c|c|c|}
\hline Oxide & \multicolumn{2}{|c|}{ LAWC26S2W } & \multicolumn{2}{c|}{ LAWC27S2W } & \multicolumn{2}{c|}{ LAWC28S2 } & \multicolumn{2}{c|}{ LAWC28S2W } \\
\hline- & Target & DCP & Target & DCP & Target & DCP & Target & DCP \\
\hline $\mathrm{Al}_{2} \mathrm{O}_{3}$ & 6.13 & 5.59 & 6.13 & 5.91 & 6.13 & 5.68 & 6.13 & 5.73 \\
\hline $\mathrm{B}_{2} \mathrm{O}_{3}$ & 13.33 & 13 & 12.25 & 11.99 & 10.1 & 9.95 & 10.1 & 9.8 \\
\hline $\mathrm{BaO}$ & 0 & 0 & 0 & 0 & 0 & 0 & 0 & 0.01 \\
\hline $\mathrm{CaO}$ & 6.41 & 5.88 & 8.57 & 7.86 & 12.89 & 10.43 & 12.89 & 10.63 \\
\hline $\mathrm{Cr}_{2} \mathrm{O}_{3}$ & 0.02 & 0.02 & 0.02 & 0.02 & 0.02 & 0.02 & 0.02 & 0.02 \\
\hline $\mathrm{Fe}_{2} \mathrm{O}_{3}$ & 0.01 & 0.08 & 0.01 & 0.06 & 0.01 & 0.06 & 0.01 & 0.05 \\
\hline $\mathrm{K}_{2} \mathrm{O}$ & 0.15 & 0.14 & 0.15 & 0.17 & 0.15 & 0.18 & 0.15 & 0.16 \\
\hline $\mathrm{Li}_{2} \mathrm{O}$ & 2.74 & 2.53 & 2.74 & 2.44 & 2.74 & 2.48 & 2.74 & 2.38 \\
\hline $\mathrm{MgO}$ & 1.51 & 1.38 & 1.51 & 1.49 & 1.51 & 1.49 & 1.51 & 1.45 \\
\hline $\mathrm{MnO}_{2}$ & 0 & 0.03 & 0 & 0.02 & 0 & 0.02 & 0 & 0.02 \\
\hline $\mathrm{Na}_{2} \mathrm{O}$ & 11.88 & 9.48 & 11.88 & 8.7 & 11.88 & 9.23 & 11.88 & 9.13 \\
\hline $\mathrm{NiO}$ & 0 & 0.01 & 0 & 0.01 & 0 & 0.01 & 0 & 0.01 \\
\hline $\mathrm{P}_{2} \mathrm{O}_{5}$ & 0.12 & 0.25 & 0.12 & 0.17 & 0 & 0.25 & 0.12 & 0.22 \\
\hline $\mathrm{SO}_{3}$ & 2.5 & 0.49 & 2.5 & 0.49 & 0 & 0.67 & 2.5 & 0.83 \\
\hline $\mathrm{SiO}_{2}$ & 50 & 47.95 & 48.92 & 47.02 & 46.77 & 43.02 & 46.77 & 43.28 \\
\hline $\mathrm{TiO}_{2}$ & 1.12 & 1.1 & 1.12 & 1.19 & 1.12 & 1.17 & 1.12 & 1.16 \\
\hline $\mathrm{ZnO}$ & 3.02 & 2.84 & 3.02 & 2.99 & 3.02 & 2.91 & 3.02 & 2.91 \\
\hline $\mathrm{Sum}$ & 101.97 & 93.81 & 101.97 & 93.72 & 99.35 & 90.52 & 101.97 & 90.93 \\
\hline $\mathrm{N}=$ & 3.02 & 3.04 & 3.02 & 3.2 & 3.02 & 2.93 & 3.02 & 3.14 \\
\hline
\end{tabular}

N/A = Not Analyzed 
Table 4.14. Viscosity and Conductivity Data for LAW Envelope A Glasses.

\begin{tabular}{|c|c|c|c|c|c|c|c|c|}
\hline Glass & LAWA108S1 & LAWA109S & LAWA110S1 & LAWA111S1 & LAWA112S1 & LAWA130 & LAWA134 & LAWA135 \\
\hline \multicolumn{9}{|c|}{ Viscosity (poise) } \\
\hline $900^{\circ} \mathrm{C}$ & 2351 & N/A & 2485 & 1887 & 912 & 1745 & 1783 & 2114 \\
\hline $950^{\circ} \mathrm{C}$ & 989 & N/A & 1015 & 615 & 416 & 784 & 764 & 927 \\
\hline $1000^{\circ} \mathrm{C}$ & 467 & N/A & 471 & 258 & 205 & 387 & 366 & 448 \\
\hline $1050^{\circ} \mathrm{C}$ & 242 & N/A & 242 & 129 & 108 & 207 & 193 & 235 \\
\hline $1100^{\circ} \mathrm{C}$ & 136 & N/A & 135 & 73 & 61 & 118 & 109 & 132 \\
\hline $1150^{\circ} \mathrm{C}$ & 81 & N/A & 81 & 46 & 36 & 71 & 66 & 78 \\
\hline $1200^{\circ} \mathrm{C}$ & 51 & N/A & 51 & 31 & 22 & 45 & 42 & 49 \\
\hline $1250^{\circ} \mathrm{C}$ & 34 & N/A & 34 & 22 & 14 & 30 & 28 & 32 \\
\hline \multicolumn{9}{|c|}{ Conductivity (S/cm) } \\
\hline $900^{\circ} \mathrm{C}$ & 0.143 & 0.131 & 0.124 & 0.091 & 0.130 & 0.130 & 0.117 & 0.11 \\
\hline $950^{\circ} \mathrm{C}$ & 0.188 & 0.170 & 0.165 & 0.124 & 0.172 & 0.173 & 0.157 & 0.146 \\
\hline $1000^{\circ} \mathrm{C}$ & 0.239 & 0.216 & 0.212 & 0.162 & 0.224 & 0.223 & 0.204 & 0.189 \\
\hline $1050^{\circ} \mathrm{C}$ & 0.296 & 0.266 & 0.265 & 0.206 & 0.286 & 0.279 & 0.257 & 0.238 \\
\hline $1100^{\circ} \mathrm{C}$ & 0.359 & 0.320 & 0.321 & 0.255 & 0.358 & 0.342 & 0.317 & 0.292 \\
\hline $1150^{\circ} \mathrm{C}$ & 0.426 & 0.378 & 0.382 & 0.309 & 0.441 & 0.410 & 0.382 & 0.352 \\
\hline $1200^{\circ} \mathrm{C}$ & 0.499 & 0.441 & 0.445 & 0.368 & 0.536 & 0.482 & 0.452 & 0.417 \\
\hline $1250^{\circ} \mathrm{C}$ & 0.575 & 0.506 & 0.511 & 0.431 & 0.644 & 0.560 & 0.527 & 0.487 \\
\hline
\end{tabular}

N/A = Not Analyzed 
Table 4.15. Viscosity and Conductivity Data for LAW Envelope B Glasses.

\begin{tabular}{|c|c|c|c|c|c|c|c|c|c|c|}
\hline Glass & LAWB60S2 & LAWB61 & LAWB62 & LAWB63 & LAWB64 & LAWB65 & LAWB66 & LAWB67 & LAWB68 & LAWB69 \\
\hline \multicolumn{11}{|c|}{ Viscosity (poise) } \\
\hline $900^{\circ} \mathrm{C}$ & 765 & 825 & 636 & 1060 & 993 & 1558 & 1214 & 2690 & 1835 & 772 \\
\hline $950^{\circ} \mathrm{C}$ & 335 & 374 & 279 & 445 & 389 & 662 & 534 & 1068 & 754 & 357 \\
\hline $1000^{\circ} \mathrm{C}$ & 167 & 187 & 137 & 213 & 180 & 314 & 259 & 482 & 349 & 182 \\
\hline $1050^{\circ} \mathrm{C}$ & 91 & 101 & 74 & 114 & 95 & 162 & 136 & 241 & 178 & 100 \\
\hline $1100^{\circ} \mathrm{C}$ & 54 & 59 & 43 & 66 & 55 & 90 & 77 & 131 & 98 & 59 \\
\hline $1150^{\circ} \mathrm{C}$ & 35 & 36 & 27 & 41 & 34 & 53 & 46 & 77 & 58 & 37 \\
\hline $1200^{\circ} \mathrm{C}$ & 23 & 23 & 18 & 27 & 23 & 33 & 29 & 47 & 36 & 24 \\
\hline $1250^{\circ} \mathrm{C}$ & 16 & 16 & 12 & 18 & 16 & 22 & 19 & 31 & 23 & 16 \\
\hline \multicolumn{11}{|c|}{ Conductivity (S/cm) } \\
\hline $950^{\circ} \mathrm{C}$ & 0.078 & 0.105 & 0.119 & 0.077 & 0.102 & 0.069 & 0.085 & 0.071 & 0.065 & 0.072 \\
\hline $1000^{\circ} \mathrm{C}$ & 0.113 & 0.144 & 0.166 & 0.108 & 0.14 & 0.099 & 0.119 & 0.101 & 0.093 & 0.107 \\
\hline $1050^{\circ} \mathrm{C}$ & 0.154 & 0.189 & 0.221 & 0.147 & 0.186 & 0.135 & 0.159 & 0.136 & 0.128 & 0.149 \\
\hline $1100^{\circ} \mathrm{C}$ & 0.202 & 0.241 & 0.282 & 0.194 & 0.239 & 0.176 & 0.208 & 0.175 & 0.168 & 0.197 \\
\hline $1150^{\circ} \mathrm{C}$ & 0.257 & 0.3 & 0.35 & 0.248 & 0.3 & 0.222 & 0.263 & 0.217 & 0.214 & 0.25 \\
\hline $1200^{\circ} \mathrm{C}$ & 0.316 & 0.364 & 0.423 & 0.309 & 0.369 & 0.272 & 0.326 & 0.261 & 0.265 & 0.308 \\
\hline $1250^{\circ} \mathrm{C}$ & 0.381 & 0.433 & 0.5 & 0.379 & 0.444 & 0.326 & 0.397 & 0.307 & 0.321 & 0.369 \\
\hline
\end{tabular}


Table 4.15. Viscosity and Conductivity Data for LAW Envelope B Glasses (cont'd).

\begin{tabular}{|c|c|c|c|c|c|c|c|c|c|c|}
\hline Glass & LAWB70 & LAWB71 & LAWB72 & LAWB73 & LAWB74 & LAWB75 & LAWB76 & LAWB77 & LAWB78 & LAWB79 \\
\hline \multicolumn{11}{|c|}{ Viscosity (poise) } \\
\hline $900^{\circ} \mathrm{C}$ & 861 & 787 & 983 & 911 & 798 & 694 & 640 & 1692 & 1422 & 1143 \\
\hline $950^{\circ} \mathrm{C}$ & 394 & 372 & 425 & 406 & 361 & 325 & 307 & 742 & 530 & 461 \\
\hline $1000^{\circ} \mathrm{C}$ & 198 & 192 & 209 & 199 & 180 & 166 & 161 & 359 & 237 & 215 \\
\hline $1050^{\circ} \mathrm{C}$ & 107 & 107 & 113 & 105 & 96 & 91 & 90 & 189 & 121 & 113 \\
\hline $1100^{\circ} \mathrm{C}$ & 62 & 63 & 67 & 60 & 55 & 54 & 54 & 107 & 68 & 65 \\
\hline $1150^{\circ} \mathrm{C}$ & 38 & 39 & 42 & 36 & 34 & 33 & 34 & 64 & 42 & 40 \\
\hline $1200^{\circ} \mathrm{C}$ & 24 & 26 & 28 & 23 & 21 & 22 & 22 & 40 & 28 & 26 \\
\hline $1250^{\circ} \mathrm{C}$ & 16 & 17 & 19 & 15 & 14 & 14 & 15 & 26 & 19 & 18 \\
\hline \multicolumn{11}{|c|}{ Conductivity (S/cm) } \\
\hline $950^{\circ} \mathrm{C}$ & 0.096 & 0.107 & 0.071 & 0.107 & 0.113 & 0.095 & 0.134 & 0.071 & 0.085 & 0.08 \\
\hline $1000^{\circ} \mathrm{C}$ & 0.129 & 0.144 & 0.103 & 0.14 & 0.154 & 0.133 & 0.186 & 0.1 & 0.114 & 0.108 \\
\hline $1050^{\circ} \mathrm{C}$ & 0.169 & 0.189 & 0.14 & 0.181 & 0.203 & 0.181 & 0.244 & 0.138 & 0.149 & 0.142 \\
\hline $1100^{\circ} \mathrm{C}$ & 0.217 & 0.24 & 0.184 & 0.229 & 0.26 & 0.241 & 0.307 & 0.185 & 0.191 & 0.183 \\
\hline $1150^{\circ} \mathrm{C}$ & 0.274 & 0.297 & 0.234 & 0.285 & 0.325 & 0.312 & 0.374 & 0.242 & 0.241 & 0.231 \\
\hline $1200^{\circ} \mathrm{C}$ & 0.34 & 0.361 & 0.289 & 0.35 & 0.397 & 0.397 & 0.444 & 0.31 & 0.299 & 0.286 \\
\hline $1250^{\circ} \mathrm{C}$ & 0.417 & 0.431 & 0.349 & 0.423 & 0.477 & 0.496 & 0.516 & 0.391 & 0.366 & 0.349 \\
\hline
\end{tabular}


Table 4.15. Viscosity and Conductivity Data for LAW Envelope B Glasses (cont'd).

\begin{tabular}{|c|c|c|c|c|c|c|c|}
\hline Glass & LAWB80 & LAWB81 & LAWB82 & LAWB83 & LAWB84 & LAWB85 & LAWB86 \\
\hline \multicolumn{8}{|c|}{ Viscosity (poise) } \\
\hline $900^{\circ} \mathrm{C}$ & 1407 & 876 & 714 & 1710 & 1489 & 1387 & 2103 \\
\hline $950^{\circ} \mathrm{C}$ & 625 & 401 & 331 & 689 & 626 & 612 & 674 \\
\hline $1000^{\circ} \mathrm{C}$ & 305 & 202 & 168 & 317 & 295 & 299 & 278 \\
\hline $1050^{\circ} \mathrm{C}$ & 162 & 110 & 92 & 162 & 153 & 159 & 137 \\
\hline $1100^{\circ} \mathrm{C}$ & 92 & 64 & 54 & 90 & 86 & 91 & 77 \\
\hline $1150^{\circ} \mathrm{C}$ & 55 & 39 & 33 & 54 & 51 & 55 & 47 \\
\hline $1200^{\circ} \mathrm{C}$ & 35 & 25 & 21 & 34 & 32 & 35 & 31 \\
\hline $1250^{\circ} \mathrm{C}$ & 23 & 17 & 14 & 22 & 21 & 23 & 22 \\
\hline \multicolumn{8}{|c|}{ Conductivity (S/cm) } \\
\hline $950^{\circ} \mathrm{C}$ & 0.058 & 0.096 & 0.087 & 0.068 & 0.075 & 0.083 & 0.076 \\
\hline $1000^{\circ} \mathrm{C}$ & 0.081 & 0.132 & 0.122 & 0.097 & 0.104 & 0.111 & 0.105 \\
\hline $1050^{\circ} \mathrm{C}$ & 0.11 & 0.178 & 0.166 & 0.133 & 0.14 & 0.146 & 0.139 \\
\hline $1100^{\circ} \mathrm{C}$ & 0.144 & 0.234 & 0.221 & 0.175 & 0.182 & 0.187 & 0.179 \\
\hline $1150^{\circ} \mathrm{C}$ & 0.183 & 0.303 & 0.288 & 0.223 & 0.23 & 0.235 & 0.225 \\
\hline $1200^{\circ} \mathrm{C}$ & 0.227 & 0.385 & 0.369 & 0.278 & 0.285 & 0.29 & 0.276 \\
\hline $1250^{\circ} \mathrm{C}$ & 0.276 & 0.482 & 0.464 & 0.338 & 0.346 & 0.352 & 0.332 \\
\hline
\end{tabular}


Table 4.15. Viscosity and Conductivity Data for LAW Envelope B Glasses (cont'd).

\begin{tabular}{|c|c|c|c|c|c|}
\hline Glass & LAWB89 & LAWB92 & LAWB93R1 & LAWB94 & LAWB95 \\
\hline \multicolumn{6}{|l|}{ Viscosity (poise) } \\
\hline $900^{\circ} \mathrm{C}$ & 1267 & 1599 & 1341 & 1359 & 1503 \\
\hline $950^{\circ} \mathrm{C}$ & 558 & 649 & 588 & 557 & 643 \\
\hline $1000^{\circ} \mathrm{C}$ & 271 & 303 & 284 & 262 & 308 \\
\hline $1050^{\circ} \mathrm{C}$ & 142 & 158 & 149 & 137 & 162 \\
\hline $1100^{\circ} \mathrm{C}$ & 80 & 89 & 83 & 78 & 92 \\
\hline $1150^{\circ} \mathrm{C}$ & 48 & 54 & 50 & 48 & 55 \\
\hline $1200^{\circ} \mathrm{C}$ & 30 & 35 & 31 & 31 & 35 \\
\hline $1250^{\circ} \mathrm{C}$ & 20 & 24 & 20 & 21 & 23 \\
\hline \multicolumn{6}{|c|}{ Conductivity (S/cm) } \\
\hline $950^{\circ} \mathrm{C}$ & 0.093 & 0.071 & 0.075 & 0.079 & 0.096 \\
\hline $1000^{\circ} \mathrm{C}$ & 0.127 & 0.1 & 0.099 & 0.109 & 0.134 \\
\hline $1050^{\circ} \mathrm{C}$ & 0.169 & 0.135 & 0.128 & 0.145 & 0.178 \\
\hline $1100^{\circ} \mathrm{C}$ & 0.217 & 0.175 & 0.163 & 0.187 & 0.227 \\
\hline $1150^{\circ} \mathrm{C}$ & 0.271 & 0.222 & 0.204 & 0.235 & 0.282 \\
\hline $1200^{\circ} \mathrm{C}$ & 0.331 & 0.274 & 0.251 & 0.289 & 0.341 \\
\hline $1250^{\circ} \mathrm{C}$ & 0.397 & 0.331 & 0.305 & 0.350 & 0.404 \\
\hline
\end{tabular}


Table 4.16. Viscosity and Conductivity Data for LAW Envelope C Glasses.

\begin{tabular}{|c|c|c|c|c|c|c|c|c|c|}
\hline Glass & LAWC26 & LAWC26S2 & LAWC27 & LAWC27S2 & LAWC28S2 & LAWC29 & LAWC30 & LAWC31 & LAWC32 \\
\hline \multicolumn{10}{|c|}{ Viscosity (poise) } \\
\hline $900^{\circ} \mathrm{C}$ & 1974 & 1200 & 1129 & 1641 & 737 & 836 & 1043 & 1018 & 849 \\
\hline $950^{\circ} \mathrm{C}$ & 658 & 613 & 511 & 689 & 326 & 390 & 459 & 448 & 399 \\
\hline $1000^{\circ} \mathrm{C}$ & 280 & 331 & 257 & 324 & 161 & 199 & 226 & 219 & 203 \\
\hline $1050^{\circ} \mathrm{C}$ & 141 & 187 & 141 & 167 & 86 & 110 & 121 & 117 & 111 \\
\hline $1100^{\circ} \mathrm{C}$ & 81 & 110 & 83 & 93 & 50 & 64 & 70 & 67 & 64 \\
\hline $1150^{\circ} \mathrm{C}$ & 51 & 67 & 51 & 55 & 31 & 40 & 43 & 41 & 39 \\
\hline $1200^{\circ} \mathrm{C}$ & 34 & 42 & 34 & 34 & 20 & 26 & 28 & 26 & 25 \\
\hline $1250^{\circ} \mathrm{C}$ & 24 & 28 & 23 & 22 & 14 & 17 & 19 & 18 & 16 \\
\hline \multicolumn{10}{|c|}{ Conductivity (S/cm) } \\
\hline $950^{\circ} \mathrm{C}$ & N/A & 0.081 & 0.090 & 0.067 & 0.078 & 0.089 & 0.114 & 0.101 & 0.104 \\
\hline $1000^{\circ} \mathrm{C}$ & N/A & 0.112 & 0.125 & 0.094 & 0.118 & 0.124 & 0.156 & 0.135 & 0.146 \\
\hline $1050^{\circ} \mathrm{C}$ & N/A & 0.151 & 0.165 & 0.126 & 0.166 & 0.166 & 0.208 & 0.175 & 0.196 \\
\hline $1100^{\circ} \mathrm{C}$ & N/A & 0.197 & 0.209 & 0.165 & 0.221 & 0.218 & 0.271 & 0.223 & 0.253 \\
\hline $1150^{\circ} \mathrm{C}$ & N/A & 0.251 & 0.256 & 0.211 & 0.283 & 0.279 & 0.347 & 0.280 & 0.317 \\
\hline $1200^{\circ} \mathrm{C}$ & N/A & 0.313 & 0.305 & 0.263 & 0.349 & 0.350 & 0.437 & 0.346 & 0.386 \\
\hline $1250^{\circ} \mathrm{C}$ & N/A & 0.385 & 0.355 & 0.321 & 0.420 & 0.431 & 0.542 & 0.421 & 0.459 \\
\hline
\end{tabular}


Table 4.17. Results of the PCT at $\mathbf{9 0}^{\circ} \mathrm{C}$ Per ASTM C1285-98 for LAW Envelope A Glasses.

(Contract Specification 2.2.2.17.2 requires the normalized mass loss for B, Na, and Si to be less than $2.0 \mathrm{~g} / \mathrm{m}^{2}$ ).

\begin{tabular}{|c|c|c|c|c|c|c|c|c|}
\hline & LAWA112B14 & LAWA112B15 & LAWA112S2 & LAWA130 & LAWA133 & LAWA134 & LAWA135 & LAWA136 \\
\hline \multicolumn{9}{|c|}{ 7-Day PCT, Stainless Steel Vessel; S/V=2000 ${ }^{-1}$ Concentration in ppm } \\
\hline B & 23.83 & 23.71 & 31.91 & 25.59 & 29.89 & 28.39 & 27.2 & 23.85 \\
\hline $\mathrm{Na}$ & 160.1 & 154.8 & 182.7 & 126.5 & 168.3 & 102.5 & 93.78 & 89.44 \\
\hline $\mathrm{Si}$ & 75.49 & 72.46 & 90.42 & 76.74 & 92.72 & 63.06 & 62.68 & 61.15 \\
\hline \multicolumn{9}{|c|}{ 7-Day PCT Normalized Concentrations (in g/L) } \\
\hline $\mathrm{B}$ & 0.78 & 0.78 & 1.06 & 0.92 & 1.08 & 0.92 & 0.87 & 0.76 \\
\hline $\mathrm{Na}$ & 1.08 & 1.04 & 1.28 & 0.92 & 1.13 & 0.78 & 0.74 & 0.71 \\
\hline Si & 0.36 & 0.35 & 0.44 & 0.36 & 0.45 & 0.3 & 0.3 & 0.3 \\
\hline $\mathrm{pH}$ & 11.14 & 11.15 & 11.18 & 10.65 & 11.14 & 10.52 & 10.43 & 10.57 \\
\hline $\mathrm{B} / \mathrm{Na}$ & 0.72 & 0.75 & 0.83 & 1 & 0.95 & 1.18 & 1.17 & 1.07 \\
\hline \multicolumn{9}{|c|}{ 7-Day PCT Normalized Mass Loss (in g/m²) } \\
\hline $\mathrm{B}$ & 0.39 & 0.39 & 0.53 & 0.46 & 0.54 & 0.46 & 0.43 & 0.38 \\
\hline $\mathrm{Na}$ & 0.54 & 0.52 & 0.64 & 0.46 & 0.57 & 0.39 & 0.37 & 0.35 \\
\hline $\mathrm{Si}$ & 0.18 & 0.18 & 0.22 & 0.18 & 0.22 & 0.15 & 0.15 & 0.15 \\
\hline \multicolumn{9}{|c|}{ 7-Day PCT Normalized Loss Rate (in g/d/m²) } \\
\hline $\mathrm{B}$ & 0.06 & 0.06 & 0.08 & 0.07 & 0.08 & 0.07 & 0.06 & 0.05 \\
\hline $\mathrm{Na}$ & 0.08 & 0.07 & 0.09 & 0.07 & 0.08 & 0.06 & 0.05 & 0.05 \\
\hline Si & 0.03 & 0.03 & 0.03 & 0.03 & 0.03 & 0.02 & 0.02 & 0.02 \\
\hline
\end{tabular}


Table 4.18. Results of the PCT at $90^{\circ} \mathrm{C}$ Per ASTM C1285-98 for LAW Envelope B Glasses.

\begin{tabular}{|c|c|c|c|c|c|c|c|}
\hline & LAWB60S4 & LAWB61 & LAWB62 & LAWB63 & LAWB64 & LAWB65 & LAWB66 \\
\hline \multicolumn{8}{|c|}{ 7-Day PCT, Stainless Steel Vessel; S/V=2000 $\mathrm{m}^{-1}$ Concentration in ppm } \\
\hline $\mathrm{B}$ & 20.05 & 24.13 & 10.02 & 11.15 & 17.25 & 17.14 & 18.11 \\
\hline $\mathrm{Na}$ & 20.97 & 27.36 & 14.47 & 14.12 & 19.89 & 19.39 & 22.2 \\
\hline $\mathrm{Si}$ & 47.19 & 63.49 & 37.81 & 37.7 & 47.79 & 46.73 & 48.55 \\
\hline \multicolumn{8}{|c|}{ 7-Day PCT Normalized Concentrations (in g/L) } \\
\hline B & 0.52 & 0.78 & 0.33 & 0.36 & 0.56 & 0.56 & 0.59 \\
\hline $\mathrm{Na}$ & 0.51 & 0.67 & 0.36 & 0.35 & 0.49 & 0.48 & 0.55 \\
\hline Si & 0.21 & 0.28 & 0.17 & 0.17 & 0.21 & 0.21 & 0.21 \\
\hline $\mathrm{pH}$ & 10.12 & 10.82 & 10.82 & 10.82 & 10.82 & 10.82 & 10.17 \\
\hline $\mathrm{B} / \mathrm{Na}$ & 1.03 & 1.16 & 0.91 & 1.04 & 1.14 & 1.16 & 1.07 \\
\hline \multicolumn{8}{|c|}{ 7-Day PCT Normalized Mass Loss (in g/m²) } \\
\hline $\mathrm{B}$ & 0.26 & 0.39 & 0.16 & 0.18 & 0.28 & 0.28 & 0.29 \\
\hline $\mathrm{Na}$ & 0.25 & 0.34 & 0.18 & 0.17 & 0.25 & 0.24 & 0.27 \\
\hline Si & 0.11 & 0.14 & 0.08 & 0.08 & 0.11 & 0.1 & 0.11 \\
\hline \multicolumn{8}{|c|}{ 7-Day PCT Normalized Loss Rate (in g/d/m²) } \\
\hline B & 0.04 & 0.06 & 0.02 & 0.03 & 0.04 & 0.04 & 0.04 \\
\hline $\mathrm{Na}$ & 0.04 & 0.05 & 0.03 & 0.02 & 0.04 & 0.03 & 0.04 \\
\hline $\mathrm{Si}$ & 0.02 & 0.02 & 0.01 & 0.01 & 0.02 & 0.01 & 0.02 \\
\hline
\end{tabular}


Table 4.18. Results of the PCT at $90^{\circ} \mathrm{C}$ Per ASTM C1285-98 for LAW Envelope B Glasses (cont'd).

\begin{tabular}{|c|c|c|c|c|c|c|c|c|}
\hline & LAWB67 & LAWB68 & LAWB69 & LAWB70 & LAWB71 & LAWB72 & LAWB73 & LAWB74 \\
\hline \multicolumn{9}{|c|}{ 7-Day PCT, Stainless Steel Vessel; S/V=2000 $\mathrm{m}^{-1}$ Concentration in ppm } \\
\hline B & 14.97 & 13.18 & 18.82 & 42.81 & 21.50 & 33.65 & 12.74 & 14.50 \\
\hline $\mathrm{Na}$ & 11.51 & 19.27 & 23.44 & 46.00 & 27.19 & 37.78 & 15.47 & 16.34 \\
\hline $\mathrm{Si}$ & 50.91 & 44.78 & 44.25 & 69.01 & 52.44 & 58.14 & 39.51 & 41.88 \\
\hline \multicolumn{9}{|c|}{ 7-Day PCT Normalized Concentrations (in g/L) } \\
\hline $\mathrm{B}$ & 0.49 & 0.50 & 0.49 & 1.12 & 0.64 & 0.88 & 0.41 & 0.45 \\
\hline $\mathrm{Na}$ & 0.28 & 0.48 & 0.48 & 0.94 & 0.55 & 0.77 & 0.38 & 0.40 \\
\hline $\mathrm{Si}$ & 0.23 & 0.20 & 0.20 & 0.31 & 0.23 & 0.26 & 0.17 & 0.19 \\
\hline $\mathrm{pH}$ & 10.24 & 10.34 & 10.36 & 10.32 & 10.28 & 10.24 & 10.25 & 10.23 \\
\hline $\mathrm{B} / \mathrm{Na}$ & 1.71 & 1.06 & 1.03 & 1.19 & 1.16 & 1.14 & 1.08 & 1.12 \\
\hline \multicolumn{9}{|c|}{ 7-Day PCT Normalized Mass Loss (in $\mathrm{g} / \mathrm{m}^{2}$ ) } \\
\hline $\mathrm{B}$ & 0.24 & 0.25 & 0.25 & 0.56 & 0.32 & 0.44 & 0.21 & 0.23 \\
\hline $\mathrm{Na}$ & 0.14 & 0.24 & 0.24 & 0.47 & 0.28 & 0.38 & 0.19 & 0.20 \\
\hline $\mathrm{Si}$ & 0.11 & 0.10 & 0.10 & 0.15 & 0.12 & 0.13 & 0.09 & 0.09 \\
\hline \multicolumn{9}{|c|}{ 7-Day PCT Normalized Loss Rate (in $\mathrm{g} / \mathrm{d} / \mathrm{m}^{2}$ ) } \\
\hline $\mathrm{B}$ & 0.03 & 0.04 & 0.04 & 0.08 & 0.05 & 0.06 & 0.03 & 0.03 \\
\hline $\mathrm{Na}$ & 0.02 & 0.03 & 0.03 & 0.07 & 0.04 & 0.05 & 0.03 & 0.03 \\
\hline $\mathrm{Si}$ & 0.02 & 0.01 & 0.01 & 0.02 & 0.02 & 0.02 & 0.01 & 0.01 \\
\hline
\end{tabular}


Table 4.18. Results of the PCT at $90^{\circ} \mathrm{C}$ Per ASTM C1285-98 for LAW Envelope B Glasses (cont'd).

\begin{tabular}{|c|c|c|c|c|c|c|c|c|}
\hline & LAWB75 & LAWB76 & LAWB77 & LAWB78 & LAWB79 & LAWB80 & LAWB81 & LAWB82 \\
\hline \multicolumn{9}{|c|}{ 7-Day PCT, Stainless Steel Vessel; S/V=2000 $\mathrm{m}^{-1}$ Concentration in ppm } \\
\hline B & 12.57 & 15.37 & 27.73 & 46.94 & 41.78 & 33.76 & 34.46 & 15.58 \\
\hline $\mathrm{Na}$ & 11.73 & 14.64 & 29.53 & 80.68 & 62.59 & 35.79 & 38.59 & 22.43 \\
\hline $\mathrm{Si}$ & 36.10 & 42.69 & 52.03 & 70.59 & 67.28 & 56.41 & 59.15 & 39.85 \\
\hline \multicolumn{9}{|c|}{ 7-Day PCT Normalized Concentrations (in g/L) } \\
\hline $\mathrm{B}$ & 0.34 & 0.42 & 0.72 & 1.23 & 1.09 & 0.88 & 0.90 & 0.50 \\
\hline $\mathrm{Na}$ & 0.29 & 0.36 & 0.60 & 1.11 & 0.98 & 0.73 & 0.79 & 0.46 \\
\hline $\mathrm{Si}$ & 0.16 & 0.19 & 0.23 & 0.32 & 0.30 & 0.25 & 0.26 & 0.19 \\
\hline $\mathrm{pH}$ & 10.16 & 10.24 & 10.04 & 10.58 & 10.35 & 10.25 & 10.24 & 10.22 \\
\hline $\mathrm{B} / \mathrm{Na}$ & 1.19 & 1.17 & 1.20 & 1.10 & 1.12 & 1.21 & 1.15 & 1.09 \\
\hline \multicolumn{9}{|c|}{ 7-Day PCT Normalized Mass Loss (in $\mathrm{g} / \mathrm{m}^{2}$ ) } \\
\hline B & 0.17 & 0.21 & 0.36 & 0.61 & 0.55 & 0.44 & 0.45 & 0.25 \\
\hline $\mathrm{Na}$ & 0.14 & 0.18 & 0.30 & 0.56 & 0.49 & 0.36 & 0.39 & 0.23 \\
\hline $\mathrm{Si}$ & 0.08 & 0.09 & 0.12 & 0.16 & 0.15 & 0.13 & 0.13 & 0.09 \\
\hline \multicolumn{9}{|c|}{ 7-Day PCT Normalized Loss Rate (in g/d/m²) } \\
\hline B & 0.02 & 0.03 & 0.05 & 0.09 & 0.08 & 0.06 & 0.06 & 0.04 \\
\hline $\mathrm{Na}$ & 0.02 & 0.03 & 0.04 & 0.08 & 0.07 & 0.05 & 0.06 & 0.03 \\
\hline $\mathrm{Si}$ & 0.01 & 0.01 & 0.02 & 0.02 & 0.02 & 0.02 & 0.02 & 0.01 \\
\hline
\end{tabular}


Table 4.18(continued). Results of the PCT at $90^{\circ} \mathrm{C}$ Per ASTM C1285-98 for LAW Envelope B Glasses (cont’d).

\begin{tabular}{|c|c|c|c|c|c|c|}
\hline & LAWB83 & LAWB84 & LAWB85 & LAWB86 & LAWB89 & LAWB90 \\
\hline \multicolumn{7}{|c|}{ 7-Day PCT, Stainless Steel Vessel; S/V=2000 $\mathrm{m}^{-1}$ Concentration in ppm } \\
\hline B & 19.06 & 21.02 & 23.29 & 48.31 & 18.6 & 19.41 \\
\hline $\mathrm{Na}$ & 21.38 & 22.72 & 20.3 & 41 & 14.08 & 27.78 \\
\hline $\mathrm{Si}$ & 52.35 & 55.73 & 55.69 & 75.22 & 58.47 & 57.26 \\
\hline \multicolumn{7}{|c|}{ 7-Day PCT Normalized Concentrations (in g/L) } \\
\hline B & 0.61 & 0.68 & 0.65 & 1.25 & 0.6 & 0.62 \\
\hline $\mathrm{Na}$ & 0.53 & 0.56 & 0.5 & 1.01 & 0.47 & 0.55 \\
\hline $\mathrm{Si}$ & 0.23 & 0.25 & 0.25 & 0.33 & 0.25 & 0.26 \\
\hline $\mathrm{pH}$ & 10.16 & 10.16 & 10.11 & 10.14 & 10.22 & 10.28 \\
\hline $\mathrm{B} / \mathrm{Na}$ & 1.16 & 1.21 & 1.3 & 1.24 & 1.28 & 1.14 \\
\hline \multicolumn{7}{|c|}{ 7-Day PCT Normalized Mass Loss (in $\mathrm{g} / \mathrm{m}^{2}$ ) } \\
\hline $\mathrm{B}$ & 0.31 & 0.34 & 0.33 & 0.63 & 0.3 & 0.31 \\
\hline $\mathrm{Na}$ & 0.26 & 0.28 & 0.25 & 0.5 & 0.23 & 0.27 \\
\hline Si & 0.12 & 0.12 & 0.12 & 0.17 & 0.13 & 0.13 \\
\hline \multicolumn{7}{|c|}{ 7-Day PCT Normalized Loss Rate (in $\mathrm{g} / \mathrm{d} / \mathrm{m}^{2}$ ) } \\
\hline $\mathrm{B}$ & 0.04 & 0.05 & 0.05 & 0.09 & 0.04 & 0.04 \\
\hline $\mathrm{Na}$ & 0.04 & 0.04 & 0.04 & 0.07 & 0.03 & 0.04 \\
\hline $\mathrm{Si}$ & 0.02 & 0.02 & 0.02 & 0.02 & 0.02 & 0.02 \\
\hline
\end{tabular}


Table 4.18. Results of the PCT at $90^{\circ} \mathrm{C}$ Per ASTM C1285-98 for LAW Envelope B Glasses (cont'd).

\begin{tabular}{|c|c|c|c|c|c|}
\hline & LAWB91 & LAWB92 & LAWB93 & LAWB94 & LAWB95 \\
\hline \multicolumn{6}{|c|}{ 7-Day PCT, Stainless Steel Vessel; S/V=2000 $\mathrm{m}^{-1}$ Concentration in ppm } \\
\hline $\mathrm{B}$ & 24.65 & 28.43 & 26.69 & 22.12 & 20.85 \\
\hline $\mathrm{Na}$ & 44.92 & 59.63 & 17.55 & 11.75 & 8.02 \\
\hline Si & 62.79 & 64.66 & 52.00 & 52.87 & 51.49 \\
\hline \multicolumn{6}{|c|}{ 7-Day PCT Normalized Concentrations (in g/L) } \\
\hline $\mathrm{B}$ & 0.79 & 0.91 & 0.86 & 0.71 & 0.67 \\
\hline $\mathrm{Na}$ & 0.69 & 0.79 & 0.50 & 0.47 & 0.44 \\
\hline Si & 0.29 & 0.30 & 0.23 & 0.23 & 0.22 \\
\hline $\mathrm{pH}$ & 10.34 & 10.38 & 10.27 & 10.76 & 10.26 \\
\hline $\mathrm{B} / \mathrm{Na}$ & 1.14 & 1.15 & 1.73 & 1.52 & 1.52 \\
\hline \multicolumn{6}{|c|}{ 7-Day PCT Normalized Mass Loss (in g/m²) } \\
\hline $\mathrm{B}$ & 0.40 & 0.46 & 0.43 & 0.36 & 0.33 \\
\hline $\mathrm{Na}$ & 0.35 & 0.40 & 0.25 & 0.23 & 0.22 \\
\hline Si & 0.14 & 0.15 & 0.11 & 0.11 & 0.11 \\
\hline \multicolumn{6}{|c|}{ 7-Day PCT Normalized Loss Rate (in g/d/m²) } \\
\hline $\mathrm{B}$ & 0.06 & 0.07 & 0.06 & 0.05 & 0.05 \\
\hline $\mathrm{Na}$ & 0.05 & 0.06 & 0.04 & 0.03 & 0.03 \\
\hline $\mathrm{Si}$ & 0.02 & 0.02 & 0.02 & 0.02 & 0.02 \\
\hline
\end{tabular}


Table 4.19. Results of the PCT at $90^{\circ} \mathrm{C}$ Per ASTM C1285-98 for LAW Envelope C Glasses.

\begin{tabular}{|c|c|c|c|c|c|c|c|c|c|c|}
\hline & C21REV2 & C22AN107 & LAWC26 & LAWC27 & LAWC28 & LAWC29 & LAWC30 & LAWC31 & LAWC32 & LAWC33 \\
\hline \multicolumn{11}{|c|}{ 7-Day PCT, Stainless Steel Vessel; S/V=2000 $\mathrm{m}^{-1}$ Concentration in ppm } \\
\hline B & 21.63 & 35.50 & 28.16 & 14.27 & 8.92 & 9.46 & 18.64 & 17.14 & 13.05 & 20.07 \\
\hline $\mathrm{Na}$ & 63.84 & 119.10 & 58.95 & 39.02 & 38.91 & 36.73 & 58.26 & 55.57 & 49.04 & 60.41 \\
\hline $\mathrm{Si}$ & 60.18 & 89.75 & 50.07 & 41.86 & 35.72 & 36.23 & 56.64 & 52.25 & 45.34 & 63.66 \\
\hline \multicolumn{11}{|c|}{ 7-Day PCT Normalized Concentrations (in g/L) } \\
\hline $\mathrm{B}$ & 0.69 & 1.14 & 0.68 & 0.38 & 0.29 & 0.30 & 0.60 & 0.55 & 0.42 & 0.64 \\
\hline $\mathrm{Na}$ & 0.72 & 1.11 & 0.66 & 0.44 & 0.44 & 0.41 & 0.66 & 0.63 & 0.55 & 0.68 \\
\hline $\mathrm{Si}$ & 0.28 & 0.41 & 0.21 & 0.18 & 0.16 & 0.16 & 0.26 & 0.24 & 0.21 & 0.29 \\
\hline $\mathrm{pH}$ & 10.27 & 10.46 & 10.82 & 10.82 & 10.82 & 10.82 & 10.82 & 10.43 & 10.58 & 10.67 \\
\hline $\mathrm{B} / \mathrm{Na}$ & 0.96 & 1.02 & 1.03 & 0.86 & 0.65 & 0.73 & 0.91 & 0.88 & 0.76 & 0.94 \\
\hline \multicolumn{11}{|c|}{ 7-Day PCT Normalized Mass Loss (in $\mathrm{g} / \mathrm{m}^{2}$ ) } \\
\hline $\mathrm{B}$ & 0.35 & 0.57 & 0.34 & 0.19 & 0.14 & 0.15 & 0.30 & 0.27 & 0.21 & 0.32 \\
\hline $\mathrm{Na}$ & 0.36 & 0.56 & 0.33 & 0.22 & 0.22 & 0.21 & 0.33 & 0.31 & 0.28 & 0.34 \\
\hline Si & 0.14 & 0.21 & 0.11 & 0.09 & 0.08 & 0.08 & 0.13 & 0.12 & 0.10 & 0.15 \\
\hline \multicolumn{11}{|c|}{ 7-Day PCT Normalized Loss Rate (in g/d/m²) } \\
\hline $\mathrm{B}$ & 0.05 & 0.08 & 0.05 & 0.03 & 0.02 & 0.02 & 0.04 & 0.04 & 0.03 & 0.05 \\
\hline $\mathrm{Na}$ & 0.05 & 0.08 & 0.05 & 0.03 & 0.03 & 0.03 & 0.05 & 0.04 & 0.04 & 0.05 \\
\hline $\mathrm{Si}$ & 0.02 & 0.03 & 0.02 & 0.01 & 0.01 & 0.01 & 0.02 & 0.02 & 0.01 & 0.02 \\
\hline
\end{tabular}


The Catholic University of America

Vitreous State Laboratory

Table 4.20. Results of Vapor Hydration Test (VHT) at $200^{\circ} \mathrm{C}$.

\begin{tabular}{|c|c|c|c|c|}
\hline Glass Name & $\begin{array}{l}\text { Alteration depth } \\
\qquad(\mu \mathrm{m})\end{array}$ & $\begin{array}{l}\text { Test duration } \\
\text { (Days) }\end{array}$ & $\begin{array}{l}\text { Rate }\left(\mathrm{g} / \mathrm{m}^{2} / \mathrm{d}\right) \\
\text { calculated for average } \\
\text { density of } 2.655 \mathrm{~g} / \mathrm{ml} \\
\text { or measured* density }\end{array}$ & $\begin{array}{c}\text { Comparison to } \\
\text { contract limit of } \\
50 \mathrm{~g} / \mathrm{m}^{2} / \mathrm{d}\end{array}$ \\
\hline LAWA102R1 & 34 & 24 & 3.8 & $7.52 \%$ \\
\hline LAWA130 & 6 & 24 & 0.7 & $1.30 \%$ \\
\hline LAWA133 & 5 & 24 & 0.6 & $1.10 \%$ \\
\hline LAWA134 & 1 & 7 & 0.4 & $0.80 \%$ \\
\hline LAWA134 & 2 & 24 & 0.4 & $0.80 \%$ \\
\hline LAWA135 & 3 & 24 & 0.3 & $0.70 \%$ \\
\hline LAWA136 & 3 & 24 & 0.3 & $0.60 \%$ \\
\hline LAWB60 & 68 & 24 & 7.5 & $15.00 \%$ \\
\hline LAWB62 & 36.7 & 24 & 4.1 & $8.10 \%$ \\
\hline LAWB63 & 71.5 & 24 & 7.9 & $15.80 \%$ \\
\hline LAWB64 & 15 & 24 & 1.7 & $3.30 \%$ \\
\hline LAWB65 & 10.4 & 24 & 1.2 & $2.30 \%$ \\
\hline LAWB66 & 17 & 24 & 1.9 & $3.76 \%$ \\
\hline LAWB67 & 15 & 24 & 1.7 & $3.30 \%$ \\
\hline LAWB69 & 128 & 24 & 14.2 & $28.32 \%$ \\
\hline LAWB68 & 18 & 24 & 2 & $4.00 \%$ \\
\hline LAWB70 & 31 & 24 & 3.4 & $6.90 \%$ \\
\hline LAWB71 & 12 & 24 & 1.3 & $2.70 \%$ \\
\hline LAWB72 & 23 & 24 & 2.5 & $5.10 \%$ \\
\hline LAWB73 & 31 & 24 & 3.4 & $6.90 \%$ \\
\hline LAWB74 & 52 & 24 & 5.8 & $11.50 \%$ \\
\hline LAWB75 & 59 & 24 & 6.5 & $13.10 \%$ \\
\hline LAWB76 & 78 & 24 & 8.6 & $17.30 \%$ \\
\hline LAWB77 & 17 & 24 & 1.9 & $3.80 \%$ \\
\hline
\end{tabular}


Table 4.20. Results of Vapor Hydration Test (VHT) at $200^{\circ} \mathrm{C}$ (cont'd).

\begin{tabular}{|c|c|c|c|c|}
\hline Glass Name & $\begin{array}{c}\text { Alteration } \\
\text { depth }(\mu \mathrm{m})\end{array}$ & $\begin{array}{l}\text { Test } \\
\text { duration } \\
\text { Days }\end{array}$ & $\begin{array}{c}\text { Rate }\left(\mathrm{g} / \mathrm{m}^{2} / \mathrm{d}\right) \text { calculated } \\
\text { for average density of } \\
2.655 \mathrm{~g} / \mathrm{ml}^{\text {or measured }} * \\
\text { density }\end{array}$ & $\begin{array}{l}\text { Comparison to } \\
\text { contract limit of } \\
50 \mathrm{~g} / \mathrm{m}^{2} / \mathrm{d}\end{array}$ \\
\hline LAWB78 & 23 & 24 & 2.5 & $5.1 \%$ \\
\hline LAWB79 & 11 & 24 & 1.2 & $2.4 \%$ \\
\hline LAWB80 & 10 & 24 & 1.1 & $2.2 \%$ \\
\hline LAWB81 & 24 & 24 & 2.7 & $5.3 \%$ \\
\hline LAWB82 & 32 & 24 & 3.5 & $7.1 \%$ \\
\hline LAWB83* & 16 & 24 & 1.8 & $3.5 \%$ \\
\hline LAWB84 & 15 & 24 & 1.7 & $3.3 \%$ \\
\hline LAWB85 & 11 & 24 & 1.2 & $2.4 \%$ \\
\hline LAWB86 & 15 & 24 & 1.7 & $3.3 \%$ \\
\hline LAWB89 & 16 & 24 & 1.8 & $3.5 \%$ \\
\hline LAWB90 & 14 & 24 & 1.5 & $3.1 \%$ \\
\hline LAWB91 & 12 & 24 & 1.3 & $2.7 \%$ \\
\hline LAWB92 & 10 & 24 & 1.1 & $2.2 \%$ \\
\hline LAWB93 & 15 & 24 & 1.7 & $3.3 \%$ \\
\hline LAWB94 & 14 & 24 & 1.5 & $3.1 \%$ \\
\hline LAWB95 & 11 & 24 & 1.2 & $2.4 \%$ \\
\hline C22AN107 & 9 & 24 & 1.0 & $2.0 \%$ \\
\hline LAWC26 & 22 & 24 & 2.4 & $4.9 \%$ \\
\hline LAWC27 & 177 & 24 & 19.6 & $39.2 \%$ \\
\hline LAWC28 & 92 & 24 & 10.2 & $20.4 \%$ \\
\hline LAWC29 & 106 & 24 & 11.7 & $23.5 \%$ \\
\hline LAWC30 & 60 & 24 & 6.6 & $13.3 \%$ \\
\hline LAWC31* & 12 & 7 & 4.6 & $9.0 \%$ \\
\hline LAWC31* & $95 \pm 80^{(1)}$ & 24 & 12 & $24.0 \%$ \\
\hline LAWC31 & 144 & 48 & 8.0 & $16 \%$ \\
\hline LAWC32 & 206 & 24 & 22.8 & $16.9 \%$ \\
\hline LAWC33 & 17 & 24 & 1.9 & $3.8 \%$ \\
\hline LAWC33 & 75 & 48 & 4.1 & $8.3 \%$ \\
\hline
\end{tabular}

(1) Glass LAWC31 was tested 6 times, 3 of which were sampled at 24 days. 
Table 4.21. Results of Heat Treatment and SEM Analysis.

\begin{tabular}{|c|c|c|c|}
\hline \multicolumn{2}{|c|}{ Sample ID } & \multirow{2}{*}{\begin{tabular}{l}
\multicolumn{1}{c}{ Preparation Summary } \\
Glass remelted at $1200^{\circ} \mathrm{C}$ for 1 \\
hour, heat treated for 20 hours at \\
$850^{\circ} \mathrm{C}$ and quenched.
\end{tabular}} & \multirow{2}{*}{$\begin{array}{l}\text { Observations } \\
\text { Amber brown glass. Augite observed near } \\
\text { meniscus. }\end{array}$} \\
\hline LAWB61 & LAWB61C85H20 & & \\
\hline LAWB64 & LAWB64C85H20 & $\begin{array}{l}\text { Glass remelted at } 1200^{\circ} \mathrm{C} \text { for } 1 \\
\text { hour, heat treated for } 20 \text { hours at } \\
850^{\circ} \mathrm{C} \text { and quenched. }\end{array}$ & $\begin{array}{l}\text { Dark lime green glass. Augite observed near } \\
\text { meniscus. }\end{array}$ \\
\hline LAWB65 & LAWB65C85H20 & $\begin{array}{l}\text { Glass remelted at } 1200^{\circ} \mathrm{C} \text { for } 1 \\
\text { hour, heat treated for } 20 \text { hours at } \\
850^{\circ} \mathrm{C} \text { and quenched. }\end{array}$ & $\begin{array}{l}\text { Amber brown glass. Augite observed near } \\
\text { meniscus. }\end{array}$ \\
\hline LAWB66 & LAWB66C85H20 & $\begin{array}{l}\text { Glass remelted at } 1200^{\circ} \mathrm{C} \text { for } 1 \\
\text { hour, heat treated for } 20 \text { hours at } \\
850^{\circ} \mathrm{C} \text { and quenched. }\end{array}$ & $\begin{array}{l}\text { Amber brown glass. Augite observed near } \\
\text { meniscus. }\end{array}$ \\
\hline LAWB67 & LAWB67C85H20 & $\begin{array}{l}\text { Glass remelted at } 1200^{\circ} \mathrm{C} \text { for } 1 \\
\text { hour, heat treated for } 20 \text { hours at } \\
850^{\circ} \mathrm{C} \text { and quenched. }\end{array}$ & $\begin{array}{l}\text { Amber brown glass. Augite and apatite } \\
\text { were detected. }\end{array}$ \\
\hline LAWB68 & LAWB68C85H20 & $\begin{array}{l}\text { Glass remelted at } 1200^{\circ} \mathrm{C} \text { for } 1 \\
\text { hour, heat treated for } 20 \text { hours at } \\
850^{\circ} \mathrm{C} \text { and quenched. }\end{array}$ & $\begin{array}{l}\text { Amber brown glass. Augite observed near } \\
\text { crucible interface and meniscus. }\end{array}$ \\
\hline \multirow{2}{*}{ LAWB70 } & LAWB70C70H20 & $\begin{array}{l}\text { Glass remelted at } 1200^{\circ} \mathrm{C} \text { for } 1 \\
\text { hour, heat treated for } 20 \text { hours at } \\
700^{\circ} \mathrm{C} \text { and quenched. }\end{array}$ & \multirow{2}{*}{$\begin{array}{l}\text { Amber brown glass, homogeneous after } \\
\text { heat-treatment at } 850^{\circ} \mathrm{C} \text {; contained very } \\
\text { small amount (less than } 0.1 \text { vol\%) of } \\
\text { augite/aegirine crystals at the crucible } \\
\text { interface. }\end{array}$} \\
\hline & LAWB70C85H20 & $\begin{array}{l}\text { Glass remelted at } 1200^{\circ} \mathrm{C} \text { for } 1 \\
\text { hour, heat treated for } 20 \text { hours at } \\
850^{\circ} \mathrm{C} \text { and quenched. }\end{array}$ & \\
\hline \multirow{2}{*}{ LAWB73 } & LAWB73C70H20 & $\begin{array}{l}\text { Glass remelted at } 1200^{\circ} \mathrm{C} \text { for } 1 \\
\text { hour, heat treated for } 20 \text { hours at } \\
700^{\circ} \mathrm{C} \text { and quenched. }\end{array}$ & \multirow{2}{*}{$\begin{array}{l}\text { Lime green glass; contained very small } \\
\text { amount (less than } 0.1 \text { vol\%) of augite - } \\
\text { aegirine crystals at the crucible interface } \\
\text { after heat treatment at } 850^{\circ} \mathrm{C} \text { and about } 2.6 \\
\text { vol\% crystals after heat treatment at } 700^{\circ} \mathrm{C} \text {. }\end{array}$} \\
\hline & LAWB73C85H20 & $\begin{array}{l}\text { Glass remelted at } 1200^{\circ} \mathrm{C} \text { for } 1 \\
\text { hour, heat treated for } 20 \text { hours at } \\
850^{\circ} \mathrm{C} \text { and quenched. }\end{array}$ & \\
\hline \multirow{2}{*}{ LAWB79 } & LAWB79C70H20 & $\begin{array}{l}\text { Glass remelted at } 1200^{\circ} \mathrm{C} \text { for } 1 \\
\text { hour, heat treated for } 20 \text { hours at } \\
700^{\circ} \mathrm{C} \text { and quenched. }\end{array}$ & \multirow{2}{*}{$\begin{array}{l}\text { Amber brown glass containing very small } \\
\text { amount (less than } 0.1 \text { vol\%) of augite - } \\
\text { aegirine crystals at the crucible interface } \\
\text { after treatment at both temperatures. }\end{array}$} \\
\hline & LAWB79C85H20 & $\begin{array}{l}\text { Glass remelted at } 1200^{\circ} \mathrm{C} \text { for } 1 \\
\text { hour, heat treated for } 20 \text { hours at } \\
850^{\circ} \mathrm{C} \text { and quenched. }\end{array}$ & \\
\hline LAWB81 & LAWB81C85H20 & $\begin{array}{l}\text { Glass remelted at } 1200^{\circ} \mathrm{C} \text { for } 1 \\
\text { hour, heat treated for } 20 \text { hours at } \\
850^{\circ} \mathrm{C} \text { and quenched. }\end{array}$ & $\begin{array}{l}\text { Amber brown glass. Only one region in } \\
\text { glass showed crystalline phases; about six } \\
\text { agate crystals and one CrZnMg spinel } \\
\text { crystal. }\end{array}$ \\
\hline
\end{tabular}


Table 4.21. Results of Heat Treatment and SEM Analysis (cont'd).

\begin{tabular}{|c|c|c|c|}
\hline & Sample ID & Preparation Summary & Observations \\
\hline LAWB82 & LAWB82C85H20 & $\begin{array}{l}\text { Glass remelted at } 1200^{\circ} \mathrm{C} \text { for } 1 \\
\text { hour, heat treated for } 20 \text { hours at } \\
850^{\circ} \mathrm{C} \text { and quenched. }\end{array}$ & $\begin{array}{l}\text { Amber brown glass. Significant } \\
\text { augite/aegirine formation along } \\
\text { crucible interface; some crystal } \\
\text { clusters observed in the bulk } \\
\text { glass along with a small amount } \\
\text { of spinel crystals. }\end{array}$ \\
\hline \multirow{2}{*}{ LAWB83 } & LAWB83C70H20 & $\begin{array}{l}\text { Glass remelted at } 1200^{\circ} \mathrm{C} \text { for } 1 \\
\text { hour, heat treated for } 20 \text { hours at } \\
700^{\circ} \mathrm{C} \text { and quenched. }\end{array}$ & $\begin{array}{l}\text { Amber brown glass. Augite } \\
\text { formation along crucible contact } \\
\text { interface. }\end{array}$ \\
\hline & LAWB83C85H20 & $\begin{array}{l}\text { Glass remelted at } 1200^{\circ} \mathrm{C} \text { for } 1 \\
\text { hour, heat treated for } 20 \text { hours at } \\
850^{\circ} \mathrm{C} \text { and quenched. }\end{array}$ & $\begin{array}{l}\text { Amber brown glass. Augite } \\
\text { formation along crucible contact } \\
\text { interface. }\end{array}$ \\
\hline \multirow{2}{*}{ LAWB84 } & LAWB84C70H20 & $\begin{array}{l}\text { Glass remelted at } 1200^{\circ} \mathrm{C} \text { for } 1 \\
\text { hour, heat treated for } 20 \text { hours at } \\
700^{\circ} \mathrm{C} \text { and quenched. }\end{array}$ & $\begin{array}{l}\text { Amber brown glass. Augite } \\
\text { formation along crucible contact } \\
\text { interface. }\end{array}$ \\
\hline & LAWB84C85H20 & $\begin{array}{l}\text { Glass remelted at } 1200^{\circ} \mathrm{C} \text { for } 1 \\
\text { hour, heat treated for } 20 \text { hours at } \\
850^{\circ} \mathrm{C} \text { and quenched. }\end{array}$ & $\begin{array}{l}\text { Amber brown glass. Augite } \\
\text { formation along crucible contact } \\
\text { interface. }\end{array}$ \\
\hline \multirow[b]{2}{*}{ LAWB85 } & LAWB85C70H20 & $\begin{array}{l}\text { Glass remelted at } 1200^{\circ} \mathrm{C} \text { for } 1 \\
\text { hour, heat treated for } 20 \text { hours at } \\
700^{\circ} \mathrm{C} \text { and quenched. }\end{array}$ & $\begin{array}{l}\text { Amber brown glass. Augite } \\
\text { formation along crucible contact } \\
\text { interface. }\end{array}$ \\
\hline & LAWB85C85H20 & $\begin{array}{l}\text { Glass remelted at } 1200^{\circ} \mathrm{C} \text { for } 1 \\
\text { hour, heat treated for } 20 \text { hours at } \\
850^{\circ} \mathrm{C} \text { and quenched. }\end{array}$ & $\begin{array}{l}\text { Amber brown glass. Spinel-like } \\
\text { crystals along crucible contact } \\
\text { interface; few crystals of the } \\
\text { augite-aegirine family in bulk } \\
\text { glass. }\end{array}$ \\
\hline LAWB86 & LAWB86C70H20 & $\begin{array}{l}\text { Glass remelted at } 1200^{\circ} \mathrm{C} \text { for } 1 \\
\text { hour, heat treated for } 20 \text { hours at } \\
700^{\circ} \mathrm{C} \text { and quenched. }\end{array}$ & $\begin{array}{l}\text { Amber brown glass. Augite } \\
\text { formation along crucible contact } \\
\text { interface. }\end{array}$ \\
\hline \multirow{2}{*}{ LAWB89 } & LAWB89C70H20 & $\begin{array}{l}\text { Glass remelted at } 1200^{\circ} \mathrm{C} \text { for } 1 \\
\text { hour, heat treated for } 20 \text { hours at } \\
700^{\circ} \mathrm{C} \text { and quenched. }\end{array}$ & \multirow{2}{*}{$\begin{array}{l}\text { Amber brown glass. Some } \\
\text { crystals (less than } 1 \text { vol\%) } \\
\text { formed along crucible contact } \\
\text { interface. }\end{array}$} \\
\hline & LAWB89C85H20 & $\begin{array}{l}\text { Glass remelted at } 1200^{\circ} \mathrm{C} \text { for } 1 \\
\text { hour, heat treated for } 20 \text { hours at } \\
850^{\circ} \mathrm{C} \text { and quenched. }\end{array}$ & \\
\hline \multirow{2}{*}{ LAWB90 } & LAWB90C70H20 & $\begin{array}{l}\text { Glass remelted at } 1200^{\circ} \mathrm{C} \text { for } 1 \\
\text { hour, heat treated for } 20 \text { hours at } \\
700^{\circ} \mathrm{C} \text { and quenched. }\end{array}$ & \multirow{2}{*}{$\begin{array}{l}\text { Amber brown glass. Some } \\
\text { crystals (less than } 1 \text { vol\%) } \\
\text { formed along crucible contact } \\
\text { interface. }\end{array}$} \\
\hline & LAWB90C85H20 & $\begin{array}{l}\text { Glass remelted at } 1200^{\circ} \mathrm{C} \text { for } 1 \\
\text { hour, heat treated for } 20 \text { hours at } \\
850^{\circ} \mathrm{C} \text { and quenched. }\end{array}$ & \\
\hline
\end{tabular}


Table 4.21. Results of Heat Treatment and SEM Analysis (cont'd).

\begin{tabular}{|c|c|c|c|}
\hline \multicolumn{2}{|r|}{ Sample ID } & Preparation Summary & Observations \\
\hline \multirow{2}{*}{ LAWB90 } & LAWB90C70H20 & $\begin{array}{l}\text { Glass remelted at } 1150^{\circ} \mathrm{C} \text { for } 1 \\
\text { hour, heat treated for } 20 \text { hours at } \\
700^{\circ} \mathrm{C} \text { and quenched. }\end{array}$ & \multirow{2}{*}{$\begin{array}{l}\text { Amber brown glass. Some } \\
\text { crystals (less than } 1 \text { vol\%) along } \\
\text { crucible contact interface. }\end{array}$} \\
\hline & LAWB90C85H20 & $\begin{array}{l}\text { Glass remelted at } 1150^{\circ} \mathrm{C} \text { for } 1 \\
\text { hour, heat treated for } 20 \text { hours at } \\
850^{\circ} \mathrm{C} \text { and quenched. }\end{array}$ & \\
\hline \multirow{2}{*}{ LAWB91 } & LAWB91C70H20 & $\begin{array}{l}\text { Glass remelted at } 1150^{\circ} \mathrm{C} \text { for } 1 \\
\text { hour, heat treated for } 20 \text { hours at } \\
700^{\circ} \mathrm{C} \text { and quenched. }\end{array}$ & \multirow{2}{*}{$\begin{array}{l}\text { Amber brown glass. Some } \\
\text { crystals (less than } 1 \text { vol\%) along } \\
\text { crucible contact interface. }\end{array}$} \\
\hline & LAWB91C85H20 & $\begin{array}{l}\text { Glass remelted at } 1150^{\circ} \mathrm{C} \text { for } 1 \\
\text { hour, heat treated for } 20 \text { hours at } \\
850^{\circ} \mathrm{C} \text { and quenched. }\end{array}$ & \\
\hline \multirow{2}{*}{ LAWB92 } & LAWB92C70H20 & $\begin{array}{l}\text { Glass remelted at } 1150^{\circ} \mathrm{C} \text { for } 1 \\
\text { hour, heat treated for } 20 \text { hours at } \\
700^{\circ} \mathrm{C} \text { and quenched. }\end{array}$ & \multirow{2}{*}{$\begin{array}{l}\text { Amber brown glass. Some } \\
\text { crystals (less than } 1 \text { vol\%) along } \\
\text { crucible contact interface. }\end{array}$} \\
\hline & LAWB92C85H20 & $\begin{array}{l}\text { Glass remelted at } 1150^{\circ} \mathrm{C} \text { for } 1 \\
\text { hour, heat treated for } 20 \text { hours at } \\
850^{\circ} \mathrm{C} \text { and quenched. }\end{array}$ & \\
\hline \multirow{2}{*}{ LAWB93 } & LAWB93C70H20 & $\begin{array}{l}\text { Glass remelted at } 1150^{\circ} \mathrm{C} \text { for } 1 \\
\text { hour, heat treated for } 20 \text { hours at } \\
700^{\circ} \mathrm{C} \text { and quenched. }\end{array}$ & \multirow{2}{*}{$\begin{array}{l}\text { Amber brown glass. Some } \\
\text { crystals (less than } 1 \text { vol\%) along } \\
\text { crucible contact interface. }\end{array}$} \\
\hline & LAWB93C85H20 & $\begin{array}{l}\text { Glass remelted at } 1150^{\circ} \mathrm{C} \text { for } 1 \\
\text { hour, heat treated for } 20 \text { hours at } \\
850^{\circ} \mathrm{C} \text { and quenched. }\end{array}$ & \\
\hline \multirow{2}{*}{ LAWB94 } & LAWB94C70H20 & $\begin{array}{l}\text { Glass remelted at } 1150^{\circ} \mathrm{C} \text { for } 1 \\
\text { hour, heat treated for } 20 \text { hours at } \\
700^{\circ} \mathrm{C} \text { and quenched. }\end{array}$ & \multirow{2}{*}{$\begin{array}{l}\text { Amber brown glass. Crystals } \\
\text { (less than } 5 \text { vol\%) along crucible } \\
\text { contact interface. }\end{array}$} \\
\hline & LAWB94C85H20 & $\begin{array}{l}\text { Glass remelted at } 1150^{\circ} \mathrm{C} \text { for } 1 \\
\text { hour, heat treated for } 20 \text { hours at } \\
850^{\circ} \mathrm{C} \text { and quenched. }\end{array}$ & \\
\hline \multirow{2}{*}{ LAWB95 } & LAWB95C70H20 & $\begin{array}{l}\text { Glass remelted at } 1150^{\circ} \mathrm{C} \text { for } 1 \\
\text { hour, heat treated for } 20 \text { hours at } \\
700^{\circ} \mathrm{C} \text { and quenched. }\end{array}$ & \multirow{2}{*}{$\begin{array}{l}\text { Amber brown glass. Some } \\
\text { crystals (less than } 1 \text { vol\%) along } \\
\text { crucible contact interface. }\end{array}$} \\
\hline & LAWB95C85H20 & $\begin{array}{l}\text { Glass remelted at } 1150^{\circ} \mathrm{C} \text { for } 1 \\
\text { hour, heat treated for } 20 \text { hours at } \\
850^{\circ} \mathrm{C} \text { and quenched. }\end{array}$ & \\
\hline
\end{tabular}


The Catholic University of America

Vitreous State Laboratory

Table 4.22. Results of K-3 Refractory Corrosion Testing.

\begin{tabular}{|c|c|c|c|c|c|}
\hline Glass Name & $\begin{array}{l}\text { Neck Corrosion } \\
\text { (inches) }\end{array}$ & $\begin{array}{l}\text { Half-Down } \\
\text { Corrosion } \\
\text { (inches) }\end{array}$ & $\begin{array}{l}\text { Penetration } \\
\text { Depth } \\
\text { (inches) }\end{array}$ & $\begin{array}{c}\text { Cracks } \\
\text { Type } A>100 \text { um } \\
\text { in width }\end{array}$ & $\begin{array}{c}\text { Cracks } \\
\text { Type B } \\
<100 \text { um in width }\end{array}$ \\
\hline LAWA111S1 & 0.0388 & 0.0031 & 0.0225 & 1 & 1 \\
\hline LAWA112S1 & 0.0448 & 0.0034 & 0.024 & 1 & 2 \\
\hline LAWA112S2 & 0.0482 & 0.0022 & 0.0235 & 2 & 1 \\
\hline LAWA126 & 0.014 & $<0.001$ & 0.0185 & 1 & 2 \\
\hline LAWA126R3 & 0.023 & 0.0025 & 0.018 & 0 & 3 \\
\hline LAWA130 & 0.019 & 0.0015 & 0.0185 & 0 & 1 \\
\hline LAWA127 & 0.0115 & 0.0035 & 0.015 & 0 & 2 \\
\hline LAWA134 & 0.022 & 0.003 & 0.0155 & 1 & 2 \\
\hline LAWA135 & 0.0165 & 0.003 & 0.0155 & 0 & 1 \\
\hline LAWA136 & 0.018 & 0.003 & 0.014 & 0 & 1 \\
\hline LAWB61 & $* *$ & $* *$ & $* *$ & 2 & 4 \\
\hline LAWB62 & $* * *$ & $* * *$ & $* * *$ & $* * *$ & $* * *$ \\
\hline LAWB63 & $* *$ & $* *$ & $* *$ & 2 & $4+(2$ corners missing $)$ \\
\hline LAWB64 & $* *$ & $* *$ & $* *$ & 6 & 5 \\
\hline LAWB65 & $* *$ & $* *$ & $* *$ & 1 & 3 \\
\hline LAWB67 & $<0.001$ & $*$ & 0.028 & 2 & 5 \\
\hline LAWB68 & 0.0055 & $*$ & 0.0255 & 4 & 1 \\
\hline LAWB69 & 0.014 & 0.0035 & 0.041 & 3 & $5+(2$ corners missing $)$ \\
\hline LAWB70 & 0.01 & 0.001 & 0.035 & 3 & $3+(2$ corners missing $)$ \\
\hline LAWB71 & 0.0095 & 0.0005 & 0.032 & 2 & $5+(2$ corners missing $)$ \\
\hline LAWB72 & 0.0075 & 0.001 & 0.0325 & 3 & 5 \\
\hline LAWB73 & 0.0095 & * & 0.034 & 6 & $3+(2$ corners missing $)$ \\
\hline LAWB74 & 0.014 & 0.0005 & 0.038 & 3 & $1+(2$ corners missing $)$ \\
\hline LAWB77 & 0.007 & 0.0015 & 0.0335 & 2 & $4+(2$ corners missing $)$ \\
\hline LAWB78 & 0.014 & 0.0015 & 0.029 & 2 & $5+(2$ corners missing $)$ \\
\hline LAWB79 & 0.013 & 0.0025 & 0.03 & 1 & $3+(2$ corners missing $)$ \\
\hline LAWB80 & 0.011 & 0.002 & 0.0305 & 1 & 6 \\
\hline LAWB81 & 0.012 & 0.0025 & 0.0335 & 2 & 1 \\
\hline LAWB82 & 0.009 & $<0.001$ & 0.025 & 2 & $3+(2$ corners missing $)$ \\
\hline LAWB83 & 0.0095 & $<0.001$ & 0.0265 & 1 & 4 \\
\hline LAWB84 & 0.0095 & $<0.001$ & 0.026 & 1 & 6 \\
\hline LAWB85 & 0.0065 & $<0.001$ & 0.029 & 0 & 3 \\
\hline LAWB86 & 0.0065 & $<0.001$ & 0.0255 & 1 & $2+(2$ corners missing $)$ \\
\hline LAWB64S0 & 0.01 & 0.0005 & 0.037 & 5 & $3+(2$ corners missing $)$ \\
\hline
\end{tabular}

* The width of the coupons increased by 1-2 mil compared to the original width.

** Measurement could not be performed due to longitudinal and transverse cracks near neck and half-down region.

*** Coupon broke off at the half-down region and fell into the molten glass.

**** A few of shallow cracks (horizontal as well as along the length of the coupon) developed. 
The Catholic University of America

Vitreous State Laboratory

Table 4.22. Results of K-3 Refractory Corrosion Testing (cont'd).

\begin{tabular}{|c|c|c|c|c|c|}
\hline Glass Name & $\begin{array}{l}\text { Neck Corrosion } \\
\text { (inches) }\end{array}$ & $\begin{array}{l}\text { Half-Down } \\
\text { Corrosion } \\
\text { (inches) }\end{array}$ & $\begin{array}{l}\text { Penetration } \\
\text { Depth } \\
\text { (inches) }\end{array}$ & $\begin{array}{l}\text { Cracks } \\
\text { Type } A>100 \text { um } \\
\text { in width }\end{array}$ & $\begin{array}{c}\text { Cracks } \\
\text { Type } \mathrm{B}<100 \text { um in } \\
\text { width }\end{array}$ \\
\hline LAWB89\# & 0.003 & 0.0005 & 0.014 & 3 & 4 \\
\hline LAWB90\# & 0.002 & $*$ & 0.011 & 3 & 2 \\
\hline LAWB91\# & 0.002 & 0.0015 & 0.0115 & 2 & 0 \\
\hline LAWB92\# & 0.003 & 0.001 & 0.01 & 0 & 2 \\
\hline LAWB89R & 0.005 & $*$ & 0.0345 & 9 & $2+(2$ corners missing $)$ \\
\hline LAWB90R & 0.0115 & 0.001 & 0.027 & 2 & $1+(2$ corners missing $)$ \\
\hline LAWB91R & 0.006 & $<0.001$ & 0.025 & 3 & 1 \\
\hline LAWB92R & 0.0125 & $<0.001$ & 0.0235 & 0 & 1 \\
\hline LAWB93 & 0.005 & $*$ & 0.028 & 2 & 1 \\
\hline LAWB94 & 0.0045 & $*$ & 0.028 & 1 & 1 \\
\hline LAWB95 & 0.006 & $*$ & 0.0315 & 2 & 1 \\
\hline LAWC27 & 0.018 & 0.002 & 0.0355 & 1 & $1 * * * *$ \\
\hline LAWC29 & 0.0135 & 0.0015 & 0.0215 & 1 & $2 * * * *$ \\
\hline LAWC31 & 0.0175 & 0.005 & 0.0185 & 1 & 3 \\
\hline LAWC31R2 & 0.018 & 0.0025 & 0.0195 & 1 & 4 \\
\hline LAWC21REV2 & 0.0195 & 0.004 & 0.0235 & 0 & 0 \\
\hline LAWC34S2 & 0.019 & 0.0015 & 0.0245 & 0 & 0 \\
\hline LAWC35S2 & 0.0185 & 0.002 & 0.0225 & 0 & $0 * * * *$ \\
\hline LAWC36S2 & 0.0155 & 0.0025 & 0.0215 & 0 & $0 * * * *$ \\
\hline
\end{tabular}

* The width of the coupons increased by 1-2 mil compared to the original width.

** Measurement could not be performed due to longitudinal and transverse cracks near neck and half-down region.

**** A few of shallow cracks (horizontal as well as along the length of the coupon) developed.

\# Test was reduced duration (2 days instead of 6 days) 
The Catholic University of America Vitreous State Laboratory

Table 4.23. Glass Transition Temperature $\left(T_{G}\right)$ Measured by Differential Thermal Analysis for LAW Glasses.

\begin{tabular}{|c|c|}
\hline Glass Name & $\mathrm{T}_{\mathrm{G}}\left({ }^{\circ} \mathrm{C}\right)$ \\
\hline LAWA88 & 494 \\
\hline LAWA88R1 & 490 \\
\hline LAWA102 & 472 \\
\hline LAWA133 & 512 \\
\hline LAWA134 & 488 \\
\hline LAWA135 & 503 \\
\hline LAWA136 & 496 \\
\hline LAWB60 & 494 \\
\hline LAWB69 & 488 \\
\hline LAWB70 & 478 \\
\hline LAWB71 & 478 \\
\hline LAWB72 & 486 \\
\hline LAWB73 & 486 \\
\hline LAWB74 & 488 \\
\hline LAWB75 & 470 \\
\hline LAWB76 & 480 \\
\hline LAWB77 & 489 \\
\hline LAWB78 & 467 \\
\hline LAWB79 & 492 \\
\hline LAWB80 & 484 \\
\hline LAWB81 & 480 \\
\hline LAWB82 & 478 \\
\hline LAWB83 & 500 \\
\hline LAWB84 & 487 \\
\hline LAWB85 & 494 \\
\hline LAWB86 & 492 \\
\hline LAWB87 & 492 \\
\hline LAWB88 & 500 \\
\hline LAWB89 & 493 \\
\hline LAWB90 & 493 \\
\hline LAWB91 & 493 \\
\hline LAWB92 & 499 \\
\hline LAWB93 & 492 \\
\hline LAWB94 & 495 \\
\hline LAWB95 & 498 \\
\hline LAWC31 & 464 \\
\hline LAWC33 & 472 \\
\hline
\end{tabular}


Table 4.24. Glass Density.

\begin{tabular}{|l|c|}
\hline \multicolumn{1}{|c|}{ Glass Name } & Density $\left(\mathrm{g} / \mathrm{ml}\right.$ at $\left.20^{\circ} \mathrm{C}\right)$ \\
\hline LAWA126 & 2.687 \\
\hline LAWB83 & 2.75 \\
\hline LAWC31 & 2.71 \\
\hline $\begin{array}{l}\text { NIST Soda Lime Glass \#1826b } \\
\text { Standard density }=2.549 \mathrm{~g} / \mathrm{ml} \text { at } 20^{\circ} \mathrm{C}\end{array}$ & 2.550 \\
\hline
\end{tabular}


The Catholic University of America

Vitreous State Laboratory
ORP-55237, Rev. 0

Baseline LAW Glass Formulation Testing

Final Report, VSL-03R3460-1, Rev. 0

4.25 Results (mg/l) from TCLP Testing (Performed at VSL; Analysis by DCP).

\begin{tabular}{|c|c|c|c|c|c|c|c|c|c|}
\hline Sample & $\begin{array}{l}\text { UTS } \\
\text { limit }\end{array}$ & $\begin{array}{c}\text { A1 } \\
\text { LAWA44 } \\
\text { For AN-105 } \\
\text { (from May 2000) }\end{array}$ & $\begin{array}{c}\text { A2 } \\
\text { LAWA126 } \\
\text { For AP-101 }\end{array}$ & $\begin{array}{c}\text { A2 } \\
\text { LAWA126 } 3 \\
\text { For AP-101 } \\
\text { (repeat) }\end{array}$ & $\begin{array}{c}\text { A3 } \\
\text { LAWA102 } \\
\text { For AN-104 }\end{array}$ & $\begin{array}{c}\text { B1 } \\
\text { LAWB83 } \\
\text { For AZ-101 }\end{array}$ & $\begin{array}{c}\text { C1 } \\
\text { LAWC22 } \\
\text { for AN-107 }\end{array}$ & $\begin{array}{c}\text { C2 } \\
\text { LAWC21 } \\
\text { For AN-102 }\end{array}$ & $\begin{array}{c}\text { C2 } \\
\text { LAWC31 } \\
\text { For AN-102 }\end{array}$ \\
\hline Ag & 0.14 & $<0.07$ & $<0.07$ & $<0.07$ & $<0.07$ & $<0.07$ & $<0.07$ & $<0.07$ & $<0.07$ \\
\hline $\mathrm{Al}$ & NA & 0.27 & 0.40 & 0.40 & 0.37 & 0.29 & 0.26 & 0.24 & 0.34 \\
\hline As & 5.00 & $<0.20$ & $<0.20$ & $<0.20$ & $<0.20$ & $<0.20$ & $<0.20$ & $<0.05$ & $<0.20$ \\
\hline B & NA & 1.434 & 2.26 & 2.14 & 1.56 & 0.86 & 1.48 & 1.48 & 1.23 \\
\hline $\mathrm{Ba}$ & 21.0 & 0.005 & $0.64^{(1)}$ & $0.64^{(1)}$ & $0.58^{(1)}$ & $0.59^{(1)}$ & $1.07^{(1)}$ & $0.92^{(1)}$ & $0.57^{(1)}$ \\
\hline $\mathrm{Ca}$ & NA & 1.161 & 1.18 & 1.15 & 1.96 & 1.44 & 2.02 & 2.14 & 1.98 \\
\hline $\mathrm{Cd}$ & 0.11 & $<0.03$ & $<0.03$ & $<0.03$ & $<0.03$ & 0.03 & $<0.03$ & $<0.03$ & $<0.03$ \\
\hline $\mathrm{Cr}$ & 0.60 & $<0.01$ & $<0.01$ & $<0.01$ & $<0.01$ & $<0.01$ & $<0.01$ & 0.04 & 0.02 \\
\hline $\mathrm{Fe}$ & NA & 0.39 & 0.21 & 0.23 & 0.7 & 0.18 & 0.24 & 0.38 & 0.35 \\
\hline $\mathrm{K}$ & NA & 0.26 & 2.51 & 2.25 & 0.49 & 0.44 & 0.74 & 0.29 & 0.24 \\
\hline $\mathrm{Li}$ & NA & $<0.05$ & $<0.05$ & $<0.05$ & 0.63 & 0.64 & 0.66 & 0.57 & 0.5 \\
\hline $\mathrm{Mg}$ & NA & 0.62 & 0.57 & 0.55 & 0.46 & 0.50 & 0.44 & 0.41 & 0.37 \\
\hline $\mathrm{Mn}$ & NA & $<0.04$ & $<0.04$ & $<0.04$ & $<0.04$ & $<0.04$ & $<.007$ & $<.007$ & $<0.04$ \\
\hline $\mathrm{Ni}$ & 11 & $<0.04$ & $<0.04$ & $<0.04$ & $<0.04$ & $<0.04$ & $<0.04$ & $<0.04$ & $<0.04$ \\
\hline $\mathrm{P}$ & NA & 0.09 & $<0.60$ & $<0.60$ & $<0.60$ & $<0.60$ & $<0.60$ & $<0.60$ & $<0.60$ \\
\hline $\mathrm{Pb}$ & 0.75 & $<0.10$ & $<0.10$ & $<0.10$ & $<0.10$ & $<0.10$ & $<0.10$ & $<.024$ & 0.16 \\
\hline Se & 5.7 & $<0.90$ & $<0.90$ & $<0.90$ & $<0.90$ & $<0.90$ & $<0.90$ & $<0.05$ & $<0.90$ \\
\hline $\mathrm{Si}$ & NA & 1.75 & 2.10 & 2.17 & 2.21 & 1.32 & 1.53 & 1.67 & 2.64 \\
\hline $\mathrm{Sr}$ & NA & 0.001 & 0.02 & 0.02 & 0.01 & 0.01 & 0.019 & 0.00 & 0.01 \\
\hline $\mathrm{Ti}$ & NA & 0.02 & 0.04 & 0.05 & 0.03 & 0.03 & 0.016 & 0.02 & 0.04 \\
\hline $\mathrm{Zn}$ & 4.3 & 1.12 & 1.64 & 1.64 & 1.17 & 1.1 & 1.12 & 1.12 & 1.16 \\
\hline $\mathrm{Zr}$ & NA & 0.04 & 0.07 & 0.08 & 0.08 & 0.04 & 0.04 & 0.06 & 0.11 \\
\hline $\mathrm{pH}$ & NA & 4.94 & 4.95 & 4.94 & 4.94 & 4.94 & 4.94 & 4.94 & 4.94 \\
\hline
\end{tabular}

(1) * Ba values in TCLP extracts are relatively high due to a suspected interference. 
The Catholic University of America Vitreous State Laboratory
Baseline LAW Glass Formulation Testing

Final Report, VSL-03R3460-1, Rev. 0

Table 5.1. Composition and Characterization Data for Glass LAWA44 for Tank AN-105 Supernatant (Batch 8) (Based on TFCOUP Rev.2) [2].

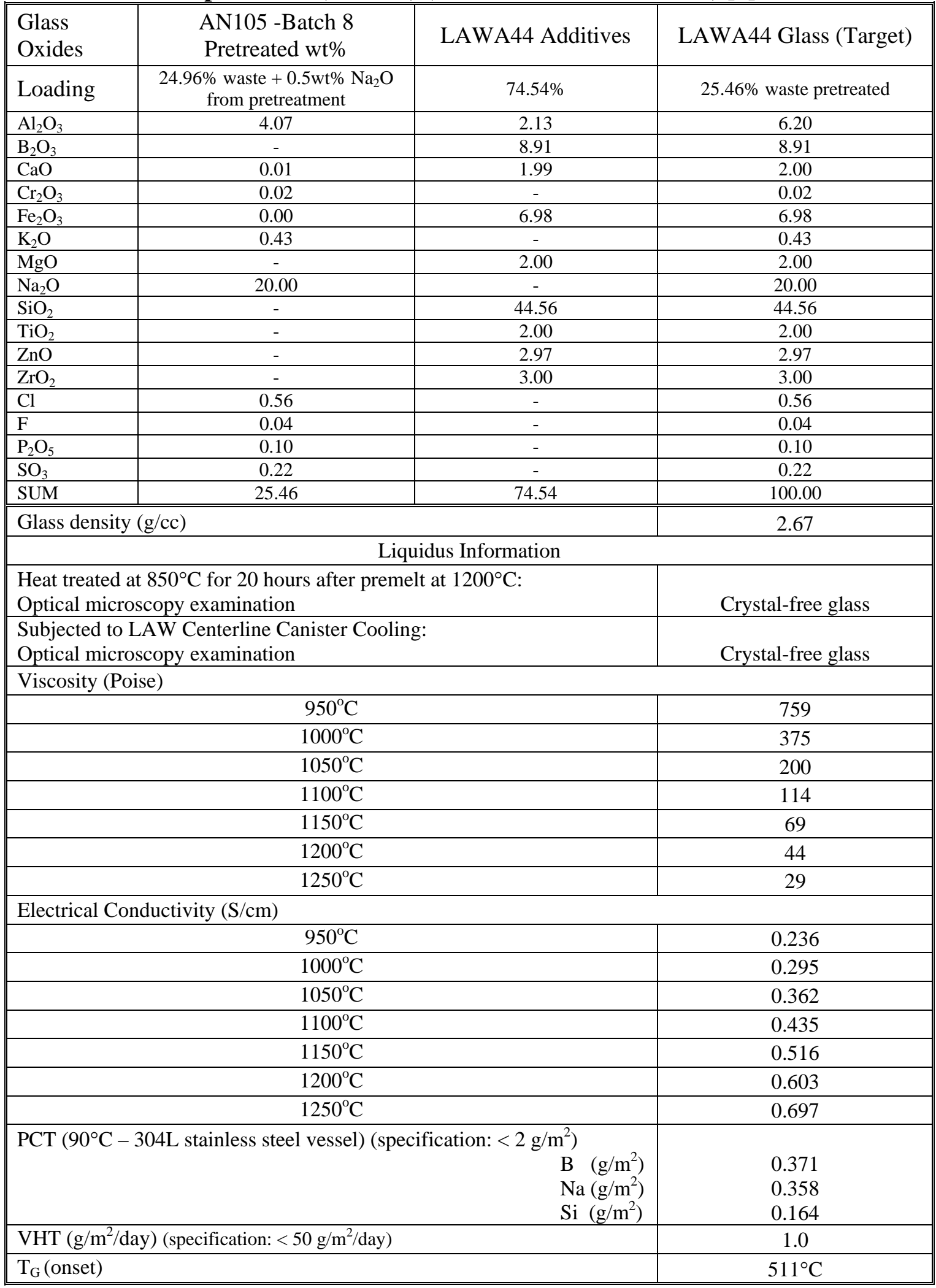


The Catholic University of America Vitreous State Laboratory
Baseline LAW Glass Formulation Testing

Final Report, VSL-03R3460-1, Rev. 0

Table 5.2. Composition and Characterization Data for Glass LAWA88 for Tank AW-101 Supernatant (Batch 14) (Based on TFCOUP Rev.2) [2].

\begin{tabular}{|c|c|c|c|}
\hline $\begin{array}{l}\text { Glass } \\
\text { Oxides }\end{array}$ & $\begin{array}{l}\text { AW101- Batch } 14 \\
\text { Pretreated wt } \%\end{array}$ & LAWA88 Additives & LAWA88 Glass (Target) \\
\hline Loading & $\begin{array}{l}25.29 \mathrm{wt} \% \text { waste }+0.5 \mathrm{wt} \% \\
\mathrm{Na}_{2} \mathrm{O} \text { from pretreatment }\end{array}$ & $74.21 \%$ & $\begin{array}{c}25.79 \mathrm{wt} \% \\
\text { Waste pretreated }\end{array}$ \\
\hline $\mathrm{Al} 2 \mathrm{O} 3$ & 3.13 & 3.15 & 6.28 \\
\hline $\mathrm{B} 2 \mathrm{O} 3$ & 0.00 & 9.76 & 9.76 \\
\hline $\mathrm{CaO}$ & 0.01 & 2.00 & 2.01 \\
\hline Cr2O3 & 0.02 & - & 0.02 \\
\hline $\mathrm{Fe} 2 \mathrm{O} 3$ & 0.00 & 5.57 & 5.57 \\
\hline $\mathrm{K} 2 \mathrm{O}$ & 1.89 & - & 1.89 \\
\hline $\mathrm{MgO}$ & 0.00 & 1.48 & 1.48 \\
\hline $\mathrm{Na} 2 \mathrm{O}$ & 20.00 & - & 20.00 \\
\hline $\mathrm{SiO} 2$ & 0.00 & 44.27 & 44.26 \\
\hline $\mathrm{TiO} 2$ & 0.00 & 2.00 & 2.00 \\
\hline $\mathrm{ZnO}$ & 0.00 & 2.97 & 2.97 \\
\hline $\mathrm{ZrO} 2$ & 0.00 & 3.01 & 3.01 \\
\hline $\mathrm{Cl}$ & 0.38 & - & 0.38 \\
\hline $\bar{F}$ & 0.10 & - & 0.10 \\
\hline P2O5 & 0.10 & - & 0.10 \\
\hline $\mathrm{SO} 3$ & 0.17 & - & 0.17 \\
\hline SUM & 25.79 & 74.21 & 100.00 \\
\hline \multicolumn{3}{|c|}{ Glass density (g/cc) } & 2.69 \\
\hline \multicolumn{4}{|c|}{ Liquidus Information } \\
\hline \multicolumn{3}{|c|}{$\begin{array}{l}\text { Heat treated at } 850^{\circ} \mathrm{C} \text { for } 20 \text { hours after premelt at } 1200^{\circ} \mathrm{C} \text { : } \\
\text { Optical microscopy examination }\end{array}$} & Crystal-free glass \\
\hline \multicolumn{3}{|c|}{$\begin{array}{l}\text { Subjected to LAW Centerline Canister Cooling: } \\
\text { Optical microscopy examination }\end{array}$} & Crystal-free glass \\
\hline \multicolumn{4}{|c|}{ Viscosity (Poise) } \\
\hline \multicolumn{3}{|c|}{$950^{\circ} \mathrm{C}$} & 569 \\
\hline \multicolumn{3}{|c|}{$1000^{\circ} \mathrm{C}$} & 294 \\
\hline \multicolumn{3}{|c|}{$1050^{\circ} \mathrm{C}$} & 163 \\
\hline \multicolumn{3}{|c|}{$1100^{\circ} \mathrm{C}$} & 96 \\
\hline \multicolumn{3}{|c|}{$1150^{\circ} \mathrm{C}$} & 60 \\
\hline \multicolumn{3}{|c|}{$1200^{\circ} \mathrm{C}$} & 39 \\
\hline \multicolumn{3}{|c|}{$1250^{\circ} \mathrm{C}$} & 26 \\
\hline \multicolumn{4}{|c|}{ Electrical Conductivity $(\mathrm{S} / \mathrm{cm})$} \\
\hline \multicolumn{3}{|c|}{$950^{\circ} \mathrm{C}$} & 0.246 \\
\hline \multicolumn{3}{|c|}{$1000^{\circ} \mathrm{C}$} & 0.314 \\
\hline \multicolumn{3}{|c|}{$1050^{\circ} \mathrm{C}$} & 0.389 \\
\hline \multicolumn{3}{|c|}{$1100^{\circ} \mathrm{C}$} & 0.472 \\
\hline \multicolumn{3}{|c|}{$1150^{\circ} \mathrm{C}$} & 0.562 \\
\hline \multicolumn{3}{|c|}{$1200^{\circ} \mathrm{C}$} & 0.658 \\
\hline \multicolumn{3}{|c|}{$1250^{\circ} \mathrm{C}$} & 0.759 \\
\hline \multicolumn{3}{|c|}{$\begin{array}{r}\text { PCT }\left(90^{\circ} \mathrm{C}-304 \mathrm{~L} \text { stainless steel vessel) (specification: }<2 \mathrm{~g} / \mathrm{m}^{2}\right) \\
\mathrm{B}\left(\mathrm{g} / \mathrm{m}^{2}\right) \\
\mathrm{Na}\left(\mathrm{g} / \mathrm{m}^{2}\right) \\
\mathrm{Si}\left(\mathrm{g} / \mathrm{m}^{2}\right)\end{array}$} & $\begin{array}{l}0.434 \\
0.426 \\
0.171\end{array}$ \\
\hline \multicolumn{3}{|c|}{ VHT (g/m²/day) (specification: $<50 \mathrm{~g} / \mathrm{m}^{2} /$ day) } & 1.3 \\
\hline \multicolumn{3}{|c|}{$\mathrm{T}_{\mathrm{G}}$ (onset) } & $494^{\circ} \mathrm{C}$ \\
\hline
\end{tabular}


The Catholic University of America Vitreous State Laboratory
Baseline LAW Glass Formulation Testing

Final Report, VSL-03R3460-1, Rev. 0

Table 5.3. Composition and Characterization Data for Glass LAWA126 for Tank AP-101 Supernatant (Batch 1) (Based on TFCOUP Rev. 3) [28].

\begin{tabular}{|c|c|c|c|}
\hline $\begin{array}{l}\text { Glass } \\
\text { Oxides }\end{array}$ & $\begin{array}{l}\text { AP-101- Batch } 1 \\
\text { pretreated }\end{array}$ & LAWA126 Additives & LAWA126 Glass (Target) \\
\hline Loading & $\begin{array}{l}24.22 \% \text { Waste }+0.46 \% \mathrm{Na}_{2} \mathrm{O} \\
\text { from pretreatment }\end{array}$ & $75.32 \%$ & $\begin{array}{c}24.68 \% \\
\text { Waste pretreated }\end{array}$ \\
\hline $\mathrm{Al}_{2} \mathrm{O}_{3}$ & 1.35 & 4.30 & 5.65 \\
\hline $\mathrm{B}_{2} \mathrm{O}_{3}$ & 0.00 & 9.84 & 9.85 \\
\hline $\mathrm{CaO}$ & & 1.99 & 1.99 \\
\hline $\mathrm{Cr}_{2} \mathrm{O}_{3}$ & 0.02 & & 0.02 \\
\hline $\mathrm{Fe}_{2} \mathrm{O}_{3}$ & & 5.55 & 5.55 \\
\hline $\mathrm{K}_{2} \mathrm{O}$ & 3.88 & & 3.88 \\
\hline $\mathrm{MgO}$ & & 1.48 & 1.48 \\
\hline $\mathrm{Na}_{2} \mathrm{O}$ & 18.46 & & 18.46 \\
\hline $\mathrm{SiO}_{2}$ & 0.03 & 44.18 & 44.21 \\
\hline $\mathrm{TiO}_{2}$ & & 2.00 & 2.00 \\
\hline $\mathrm{ZnO}$ & & 2.96 & 2.96 \\
\hline $\mathrm{ZrO}_{2}$ & & 3.00 & 3.00 \\
\hline $\mathrm{Cl}$ & 0.20 & & 0.20 \\
\hline $\mathrm{F}$ & 0.30 & & 0.30 \\
\hline $\mathrm{P}_{2} \mathrm{O}_{5}$ & 0.08 & & 0.08 \\
\hline $\mathrm{SO}_{3}$ & 0.35 & & 0.35 \\
\hline SUM & 24.68 & 75.32 & 100.00 \\
\hline \multicolumn{3}{|c|}{ Glass density (g/cc) } & 2.69 \\
\hline \multicolumn{4}{|c|}{ Liquidus Information [28] } \\
\hline \multicolumn{3}{|c|}{$\begin{array}{l}\text { Heat treated at } 850^{\circ} \mathrm{C} \text { for } 20 \text { hours after premelt at } 1200^{\circ} \mathrm{C} \text { : } \\
\text { Optical microscopy examination }\end{array}$} & Crystal-free glass \\
\hline \multicolumn{3}{|c|}{$\begin{array}{l}\text { Subjected to LAW Centerline Canister Cooling: } \\
\text { Optical microscopy examination }\end{array}$} & Crystal-free glass \\
\hline \multicolumn{4}{|c|}{ Viscosity (Poise) [28] } \\
\hline \multicolumn{3}{|c|}{$950^{\circ} \mathrm{C}$} & 696 \\
\hline \multicolumn{3}{|c|}{$1000^{\circ} \mathrm{C}$} & 343 \\
\hline \multicolumn{3}{|c|}{$1050^{\circ} \mathrm{C}$} & 182 \\
\hline \multicolumn{3}{|c|}{$1100^{\circ} \mathrm{C}$} & 104 \\
\hline \multicolumn{3}{|c|}{$1150^{\circ} \mathrm{C}$} & 63 \\
\hline \multicolumn{3}{|c|}{$1200^{\circ} \mathrm{C}$} & 40 \\
\hline \multicolumn{3}{|c|}{$1250^{\circ} \mathrm{C}$} & 26 \\
\hline \multicolumn{4}{|c|}{\begin{tabular}{|l} 
Electrical Conductivity (S/cm) [28] \\
\end{tabular}} \\
\hline \multicolumn{3}{|c|}{$950^{\circ} \mathrm{C}$} & 0.170 \\
\hline \multicolumn{3}{|c|}{$1000^{\circ} \mathrm{C}$} & 0.215 \\
\hline \multicolumn{3}{|c|}{$1050^{\circ} \mathrm{C}$} & 0.267 \\
\hline \multicolumn{3}{|c|}{$1100^{\circ} \mathrm{C}$} & 0.327 \\
\hline \multicolumn{3}{|c|}{$1150^{\circ} \mathrm{C}$} & 0.395 \\
\hline \multicolumn{3}{|c|}{$1200^{\circ} \mathrm{C}$} & 0.471 \\
\hline \multicolumn{3}{|c|}{$1250^{\circ} \mathrm{C}$} & 0.555 \\
\hline \multicolumn{3}{|c|}{$\begin{aligned} \text { PCT }\left(90^{\circ} \mathrm{C}-304 \mathrm{~L} \text { stainless steel vessel) (specification: }<2 \mathrm{~g} / \mathrm{m}^{2}\right)[28] \\
\mathrm{B}\left(\mathrm{g} / \mathrm{m}^{2}\right) \\
\mathrm{Na}\left(\mathrm{g} / \mathrm{m}^{2}\right) \\
\mathrm{Si}\left(\mathrm{g} / \mathrm{m}^{2}\right)\end{aligned}$} & $\begin{array}{l}0.598 \\
0.524 \\
0.166\end{array}$ \\
\hline \multicolumn{3}{|c|}{ VHT (g/m²/day) (specification: $<50 \mathrm{~g} / \mathrm{m}^{2} /$ day) [28] } & 2.5 \\
\hline \multicolumn{3}{|l|}{$\mathrm{T}_{\mathrm{G}}$ (onset) } & $496^{\circ} \mathrm{C}$ \\
\hline
\end{tabular}


The Catholic University of America Vitreous State Laboratory
Baseline LAW Glass Formulation Testing

Final Report, VSL-03R3460-1, Rev. 0

Table 5.4. Composition and Characterization Data for Glass LAWA102 for Tank AN-104 Supernatant (Batch 5) (Based on TFCOUP Rev.2) [2].

\begin{tabular}{|c|c|c|c|}
\hline $\begin{array}{l}\text { Glass } \\
\text { Oxides }\end{array}$ & $\begin{array}{c}\text { AN-104 - Batch } 5 \\
\text { Pretreated +SO3 recycled }\end{array}$ & $\begin{array}{c}\text { LAWA102 } \\
\text { additives }\end{array}$ & $\begin{array}{c}\text { LAWA102 } \\
\text { Glass (Target) }\end{array}$ \\
\hline Loading & $\begin{array}{l}17.8 \mathrm{wt} \% \text { waste }+0.42 \mathrm{wt} \% \mathrm{Na}_{2} \mathrm{O}+ \\
0.09 \mathrm{wt} \% \mathrm{SO}_{3} \text { (preatement /recycle) }\end{array}$ & $81.68 \%$ & $18.32 \%$ waste pretreated \\
\hline $\mathrm{Al}_{2} \mathrm{O}_{3}$ & 2.63 & 3.42 & 6.05 \\
\hline $\mathrm{B}_{2} \mathrm{O}_{3}$ & 0.00 & 10.01 & 10.01 \\
\hline $\mathrm{CaO}$ & 0.00 & 5.06 & 5.07 \\
\hline $\mathrm{Cr}_{2} \mathrm{O}_{3}$ & 0.02 & - & 0.02 \\
\hline $\mathrm{Fe}_{2} \mathrm{O}_{3}$ & 0.00 & 5.41 & 5.41 \\
\hline $\mathrm{K}_{2} \mathrm{O}$ & 0.26 & - & 0.26 \\
\hline $\mathrm{Li}_{2} \mathrm{O}$ & 0.00 & 2.50 & 2.50 \\
\hline $\mathrm{MgO}$ & 0.00 & 1.49 & 1.49 \\
\hline $\mathrm{Na}_{2} \mathrm{O}$ & 14.48 & - & 14.48 \\
\hline $\mathrm{SiO}_{2}$ & 0.00 & 46.56 & 46.56 \\
\hline $\mathrm{TiO}_{2}$ & 0.00 & 1.14 & 1.14 \\
\hline $\mathrm{ZnO}$ & 0.00 & 3.06 & 3.06 \\
\hline $\mathrm{ZrO}_{2}$ & 0.00 & 3.02 & 3.02 \\
\hline $\mathrm{Cl}$ & 0.33 & - & 0.33 \\
\hline $\mathrm{F}$ & 0.03 & - & 0.03 \\
\hline $\mathrm{P}_{2} \mathrm{O}_{5}$ & 0.13 & - & 0.13 \\
\hline $\mathrm{SO}_{3}$ & 0.44 & - & 0.44 \\
\hline SUM & 18.32 & 81.68 & 100.00 \\
\hline \multicolumn{3}{|c|}{ "Glass density (g/cc) } & 2.61 \\
\hline \multicolumn{4}{|c|}{ Liquidus Information } \\
\hline \multicolumn{3}{|c|}{$\begin{array}{l}\text { Heat treated at } 850^{\circ} \mathrm{C} \text { for } 20 \text { hours after premelt at } 1200^{\circ} \mathrm{C} \text { : } \\
\text { Optical microscopy examination }\end{array}$} & Crystal-free glass \\
\hline \multicolumn{3}{|c|}{$\begin{array}{l}\text { Subjected to LAW Centerline Canister Cooling: } \\
\text { Optical microscopy examination }\end{array}$} & Crystal-free glass \\
\hline \multicolumn{4}{|c|}{ Viscosity (Poise) } \\
\hline \multicolumn{3}{|c|}{$950^{\circ} \mathrm{C}$} & 759 \\
\hline \multicolumn{3}{|c|}{$1000^{\circ} \mathrm{C}$} & 375 \\
\hline \multicolumn{3}{|c|}{$1050^{\circ} \mathrm{C}$} & 200 \\
\hline \multicolumn{3}{|c|}{$1100^{\circ} \mathrm{C}$} & 114 \\
\hline \multicolumn{3}{|c|}{$1150^{\circ} \mathrm{C}$} & 69 \\
\hline \multicolumn{3}{|c|}{$1200^{\circ} \mathrm{C}$} & 44 \\
\hline \multicolumn{3}{|c|}{$1250^{\circ} \mathrm{C}$} & 29 \\
\hline \multicolumn{4}{|c|}{ Electrical Conductivity $(\mathrm{S} / \mathrm{cm})$} \\
\hline \multicolumn{3}{|c|}{$950^{\circ} \mathrm{C}$} & 0.236 \\
\hline \multicolumn{3}{|c|}{$1000^{\circ} \mathrm{C}$} & 0.295 \\
\hline \multicolumn{3}{|c|}{$1050^{\circ} \mathrm{C}$} & 0.362 \\
\hline \multicolumn{3}{|c|}{$1100^{\circ} \mathrm{C}$} & 0.435 \\
\hline \multicolumn{3}{|c|}{$1150^{\circ} \mathrm{C}$} & 0.516 \\
\hline \multicolumn{3}{|c|}{$1200^{\circ} \mathrm{C}$} & 0.603 \\
\hline \multicolumn{3}{|c|}{$1250^{\circ} \mathrm{C}$} & 0.697 \\
\hline \multicolumn{3}{|c|}{$\begin{array}{r}\mathrm{B}\left(\mathrm{g} / \mathrm{m}^{2}\right) \\
\mathrm{Na}\left(\mathrm{g} / \mathrm{m}^{2}\right) \\
\mathrm{Si}\left(\mathrm{g} / \mathrm{m}^{2}\right)\end{array}$} & $\begin{array}{l}0.275 \\
0.222 \\
0.164\end{array}$ \\
\hline \multicolumn{3}{|c|}{ VHT (g/m²/day) (specification: $<50 \mathrm{~g} / \mathrm{m}^{2} /$ day) } & 9.6 \\
\hline \multicolumn{3}{|c|}{$\mathrm{T}_{\mathrm{G}}$ (onset) } & $476^{\circ} \mathrm{C}$ \\
\hline
\end{tabular}


The Catholic University of America Vitreous State Laboratory
Baseline LAW Glass Formulation Testing

Final Report, VSL-03R3460-1, Rev. 0

Table 5.5. Composition and Characterization Data for Glass LAWB83 for Tank AZ-101 Supernatant (Batch 2a) (Based on TFCOUP Rev.2).

\begin{tabular}{|c|c|c|c|}
\hline \begin{tabular}{|l} 
Glass \\
Oxides
\end{tabular} & $\begin{array}{l}\text { AZ-101 pretreated } \\
\quad+\mathrm{SO}_{3} \text { recycled }\end{array}$ & LAWB83 Additives & LAWB83 Glass (Target) \\
\hline Loading & $\begin{array}{c}6.66 \mathrm{wt} \% \text { waste+ } 0.37 \mathrm{wt} \% \mathrm{Na}_{2} \mathrm{O}+0.13 \\
\mathrm{wt} \% \mathrm{SO}_{3} \text { (preatement /recycle) }\end{array}$ & $92.83 \%$ & $7.17 \%$ waste pretreated \\
\hline $\mathrm{Al}_{2} \mathrm{O}_{3}$ & 0.71 & 5.48 & 6.18 \\
\hline $\mathrm{B}_{2} \mathrm{O}_{3}$ & 0.00 & 10.03 & 10.03 \\
\hline $\mathrm{CaO}$ & 0.00 & 6.78 & 6.78 \\
\hline $\mathrm{Cr}_{2} \mathrm{O}_{3}$ & 0.04 & - & 0.04 \\
\hline $\mathrm{Fe}_{2} \mathrm{O}_{3}$ & 0.00 & 5.29 & 5.29 \\
\hline $\mathrm{K}_{2} \mathrm{O}$ & 0.19 & - & 0.19 \\
\hline $\mathrm{Li}_{2} \mathrm{O}$ & - & 4.31 & 4.31 \\
\hline $\mathrm{MgO}$ & - & 2.97 & 2.97 \\
\hline $\mathrm{Na}_{2} \mathrm{O}$ & 5.47 & - & 5.47 \\
\hline $\mathrm{SiO}_{2}$ & - & 48.60 & 48.60 \\
\hline $\mathrm{TiO}_{2}$ & - & 1.39 & 1.39 \\
\hline $\mathrm{ZnO}$ & - & 4.84 & 4.84 \\
\hline $\mathrm{ZrO}_{2}$ & - & 3.16 & 3.16 \\
\hline $\mathrm{Cl}$ & 0.01 & - & 0.01 \\
\hline $\mathrm{F}$ & 0.06 & - & 0.06 \\
\hline $\mathrm{P}_{2} \mathrm{O}_{5}$ & 0.04 & - & 0.04 \\
\hline $\mathrm{SO}_{3}$ & 0.65 & - & 0.65 \\
\hline SUM & 7.17 & 92.83 & 100.00 \\
\hline \multicolumn{3}{|c|}{ Glass density (g/cc) } & 2.75 \\
\hline \multicolumn{4}{|c|}{ Liquidus Information } \\
\hline \multicolumn{3}{|c|}{$\begin{array}{l}\text { Heat treated at } 850^{\circ} \mathrm{C} \text { or at } 750^{\circ} \mathrm{C} \text { for } 20 \text { hours after premelt at } 1200^{\circ} \mathrm{C} \text { : } \\
\text { Optical microscopy examination }\end{array}$} & $\begin{array}{l}\text { Trace amount of augite along } \\
\text { crucible contact }\end{array}$ \\
\hline \multicolumn{3}{|c|}{$\begin{array}{l}\text { Subjected to LAW Centerline Canister Cooling: } \\
\text { Optical microscopy examination [32] }\end{array}$} & $\begin{aligned} &<0.4 \text { vol. } \% \text { augite near } \mathrm{Pt} \\
&+ \text { trace spinel; }<0.1 \text { vol.\% bulk }\end{aligned}$ \\
\hline \multicolumn{4}{|c|}{\begin{tabular}{|l|} 
Viscosity (Poise) \\
\end{tabular}} \\
\hline \multicolumn{3}{|c|}{$950^{\circ} \mathrm{C}$} & 689 \\
\hline \multicolumn{3}{|c|}{$1000^{\circ} \mathrm{C}$} & 317 \\
\hline \multicolumn{3}{|c|}{$1050^{\circ} \mathrm{C}$} & 162 \\
\hline \multicolumn{3}{|c|}{$1100^{\circ} \mathrm{C}$} & 90 \\
\hline \multicolumn{3}{|c|}{$1150^{\circ} \mathrm{C}$} & 54 \\
\hline \multicolumn{3}{|c|}{$1200^{\circ} \mathrm{C}$} & 34 \\
\hline \multicolumn{3}{|c|}{$1250^{\circ} \mathrm{C}$} & 22 \\
\hline \multicolumn{4}{|c|}{ Electrical Conductivity (S/cm) } \\
\hline \multicolumn{3}{|c|}{$950^{\circ} \mathrm{C}$} & 0.068 \\
\hline \multicolumn{3}{|c|}{$1000^{\circ} \mathrm{C}$} & 0.097 \\
\hline \multicolumn{3}{|c|}{$1050^{\circ} \mathrm{C}$} & 0.133 \\
\hline \multicolumn{3}{|c|}{$1100^{\circ} \mathrm{C}$} & 0.175 \\
\hline \multicolumn{3}{|c|}{$1150^{\circ} \mathrm{C}$} & 0.223 \\
\hline \multicolumn{3}{|c|}{$1200^{\circ} \mathrm{C}$} & 0.278 \\
\hline \multicolumn{3}{|c|}{$1250^{\circ} \mathrm{C}$} & 0.338 \\
\hline \multicolumn{3}{|c|}{ PCT $\left(90^{\circ} \mathrm{C}-304 \mathrm{~L}\right.$ stainless steel vessel) (specification: $\left.<2 \mathrm{~g} / \mathrm{m}^{2}\right)$} & \\
\hline \multirow{3}{*}{\multicolumn{3}{|c|}{$\begin{aligned} \mathrm{B}\left(\mathrm{g} / \mathrm{m}^{2}\right) \\
\mathrm{Na}\left(\mathrm{g} / \mathrm{m}^{2}\right) \\
\mathrm{Si}\left(\mathrm{g} / \mathrm{m}^{2}\right)\end{aligned}$}} & 0.306 \\
\hline & & & 0.263 \\
\hline & & & 0.115 \\
\hline \multicolumn{3}{|c|}{ VHT (g/m²/day) (specification: $<50 \mathrm{~g} / \mathrm{m}^{2} /$ day) } & 1.8 \\
\hline \multicolumn{3}{|c|}{$\mathrm{T}_{\mathrm{G}}$ (onset) } & $500^{\circ} \mathrm{C}$ \\
\hline
\end{tabular}


The Catholic University of America Vitreous State Laboratory

Table 5.6. Composition and Characterization Data for Glass LAWC22 for Tank AN-107 Supernatant (Batch 7) (Based on TFCOUP Rev.2) [2].

\begin{tabular}{|c|c|c|c|}
\hline $\begin{array}{l}\text { Glass } \\
\text { Oxides }\end{array}$ & $\begin{array}{l}\text { AN107 Batch } 7 \\
\text { pretreated }\end{array}$ & $\begin{array}{l}\text { LAWC22 } \\
\text { Additives }\end{array}$ & $\begin{array}{c}\text { LAWC22 } \\
\text { Glass (Target) }\end{array}$ \\
\hline Loading & $\begin{array}{l}13.83 \% \text { waste }+2.16 \mathrm{wt} \% \mathrm{Na}_{2} \mathrm{O} \text { from } \\
\text { pretreatment }\end{array}$ & $83.91 \%$ & 16.09 wt\% waste pretreated \\
\hline $\mathrm{Al}_{2} \mathrm{O}_{3}$ & 0.56 & 5.54 & 6.10 \\
\hline $\mathrm{B}_{2} \mathrm{O}_{3}$ & 0.00 & 10.07 & 10.07 \\
\hline $\mathrm{CaO}$ & 0.03 & 5.08 & 5.11 \\
\hline $\mathrm{Cr}_{2} \mathrm{O}_{3}$ & 0.02 & - & 0.02 \\
\hline $\mathrm{Fe}_{2} \mathrm{O}_{3}$ & 0.17 & 5.41 & 5.58 \\
\hline $\mathrm{K}_{2} \mathrm{O}$ & 0.09 & - & 0.09 \\
\hline $\mathrm{Li}_{2} \mathrm{O}$ & 0.00 & 2.51 & 2.51 \\
\hline $\mathrm{MgO}$ & 0.00 & 1.51 & 1.51 \\
\hline $\mathrm{Na}_{2} \mathrm{O}$ & 14.42 & 0.00 & 14.42 \\
\hline $\mathrm{NiO}$ & 0.03 & - & 0.03 \\
\hline $\mathrm{PbO}$ & 0.02 & - & 0.02 \\
\hline $\mathrm{SiO}_{2}$ & 0.00 & 46.57 & 46.57 \\
\hline $\mathrm{TiO}_{2}$ & 0.00 & 1.14 & 1.14 \\
\hline $\mathrm{ZnO}$ & 0.00 & 3.06 & 3.06 \\
\hline $\mathrm{ZrO}_{2}$ & 0.00 & 3.02 & 3.02 \\
\hline $\mathrm{Cl}$ & 0.08 & - & 0.08 \\
\hline $\mathrm{F}$ & 0.14 & - & 0.14 \\
\hline $\mathrm{P}_{2} \mathrm{O}_{5}$ & 0.12 & - & 0.12 \\
\hline $\mathrm{SO}_{3}$ & 0.31 & - & 0.31 \\
\hline SUM & 16.09 & 83.91 & 100.00 \\
\hline \multicolumn{3}{|c|}{ Glass density (g/cc) } & 2.67 \\
\hline \multicolumn{4}{|c|}{ Liquidus Information } \\
\hline \multicolumn{3}{|c|}{$\begin{array}{l}\text { Heat treated at } 850^{\circ} \mathrm{C} \text { or at } 750^{\circ} \mathrm{C} \text { for } 20 \text { hours after premelt at } 1200^{\circ} \mathrm{C} \text { : } \\
\text { Optical microscopy examination }\end{array}$} & Crystal-free glass \\
\hline \multicolumn{3}{|c|}{$\begin{array}{l}\text { Subjected to LAW Centerline Canister Cooling: } \\
\text { Optical microscopy examination }\end{array}$} & Not available \\
\hline \multicolumn{4}{|c|}{ Viscosity (Poise) } \\
\hline \multicolumn{3}{|c|}{$950^{\circ} \mathrm{C}$} & 316 \\
\hline \multicolumn{3}{|c|}{$1000^{\circ} \mathrm{C}$} & 170 \\
\hline \multicolumn{3}{|c|}{$1050^{\circ} \mathrm{C}$} & 98 \\
\hline \multicolumn{3}{|c|}{$1100^{\circ} \mathrm{C}$} & 60 \\
\hline \multicolumn{3}{|c|}{$1150^{\circ} \mathrm{C}$} & 39 \\
\hline \multicolumn{3}{|c|}{$1200^{\circ} \mathrm{C}$} & 26 \\
\hline \multicolumn{3}{|c|}{$1250^{\circ} \mathrm{C}$} & 18 \\
\hline \multicolumn{4}{|c|}{ Electrical Conductivity $(\mathrm{S} / \mathrm{cm})$} \\
\hline \multicolumn{3}{|c|}{$950^{\circ} \mathrm{C}$} & 0.180 \\
\hline \multicolumn{3}{|c|}{$1000^{\circ} \mathrm{C}$} & 0.242 \\
\hline \multicolumn{3}{|c|}{$1050^{\circ} \mathrm{C}$} & 0.309 \\
\hline \multicolumn{3}{|c|}{$1100^{\circ} \mathrm{C}$} & 0.378 \\
\hline \multicolumn{3}{|c|}{$1150^{\circ} \mathrm{C}$} & 0.448 \\
\hline \multicolumn{3}{|c|}{$1200^{\circ} \mathrm{C}$} & 0.518 \\
\hline \multicolumn{3}{|c|}{$1250^{\circ} \mathrm{C}$} & 0.588 \\
\hline \multicolumn{3}{|c|}{$\begin{array}{r}\text { PCT }\left(90^{\circ} \mathrm{C}-304 \mathrm{~L} \text { stainless steel vessel) (specification: }<2 \mathrm{~g} / \mathrm{m}^{2}\right) \\
\mathrm{B}\left(\mathrm{g} / \mathrm{m}^{2}\right) \\
\mathrm{Na}\left(\mathrm{g} / \mathrm{m}^{2}\right) \\
\mathrm{Si}\left(\mathrm{g} / \mathrm{m}^{2}\right)\end{array}$} & $\begin{array}{l}0.518 \\
0.469 \\
0.181\end{array}$ \\
\hline \multicolumn{3}{|c|}{ VHT (g/m²/day) (specification: $<50 \mathrm{~g} / \mathrm{m}^{2} /$ day) } & 1.0 \\
\hline \multicolumn{3}{|l|}{$\mathrm{T}_{\mathrm{G}}$ (onset) } & $481^{\circ} \mathrm{C}$ \\
\hline
\end{tabular}


The Catholic University of America Vitreous State Laboratory

Table 5.7. Composition and Characterization Data for Glass LAWC31 for Tank AN-102 Supernatant (Batch 3) (Based on TFCOUP Rev.2).

\begin{tabular}{|c|c|c|c|}
\hline $\begin{array}{l}\text { Glass } \\
\text { Oxides }\end{array}$ & $\begin{array}{l}\mathrm{AN}-102-\mathrm{Batch} 3 \\
\text { pretreated and } \mathrm{SO}_{3} \text { recycled }\end{array}$ & $\begin{array}{l}\text { LAWC31 } \\
\text { Additives }\end{array}$ & $\begin{array}{l}\text { LAWC31 } \\
\text { Glass (Target) }\end{array}$ \\
\hline Loading & $\begin{array}{c}11.82 \mathrm{wt} \% \text { waste }+1.85 \mathrm{Na}_{2} \mathrm{O}+0.09 \mathrm{SO}_{3} \\
\text { (preatement /recycle) }\end{array}$ & $86.24 \%$ & $13.76 \%$ waste pretreated \\
\hline $\mathrm{Al}_{2} \mathrm{O}_{3}$ & 0.86 & 5.26 & 6.12 \\
\hline $\mathrm{B}_{2} \mathrm{O}_{3}$ & 0.00 & 10.05 & 10.05 \\
\hline $\mathrm{CaO}$ & 0.03 & 7.38 & 7.41 \\
\hline $\mathrm{Cr}_{2} \mathrm{O}_{3}$ & 0.02 & - & 0.02 \\
\hline Fe2O3 & 0.01 & 4.42 & 4.42 \\
\hline $\mathrm{K}_{2} \mathrm{O}$ & 0.14 & - & 0.14 \\
\hline $\mathrm{Li}_{2} \mathrm{O}$ & - & 2.73 & 2.73 \\
\hline $\mathrm{MgO}$ & - & 1.50 & 1.50 \\
\hline $\mathrm{Na}_{2} \mathrm{O}$ & 11.96 & - & 11.96 \\
\hline $\mathrm{SiO}_{2}$ & - & 46.74 & 46.74 \\
\hline $\mathrm{TiO}_{2}$ & - & 1.12 & 1.12 \\
\hline $\mathrm{ZnO}$ & - & 4.02 & 4.02 \\
\hline $\mathrm{ZrO}_{2}$ & - & 3.02 & 3.02 \\
\hline $\mathrm{Cl}$ & 0.11 & - & 0.11 \\
\hline $\mathrm{F}$ & 0.05 & - & 0.05 \\
\hline $\mathrm{P}_{2} \mathrm{O}_{5}$ & 0.11 & 0.00 & 0.11 \\
\hline $\mathrm{SO}_{3}$ & 0.48 & - & 0.48 \\
\hline SUM & 13.76 & 86.24 & 100.00 \\
\hline \multicolumn{3}{|c|}{ Glass density (g/cc) } & 2.71 \\
\hline \multicolumn{4}{|c|}{ Liquidus Information } \\
\hline \multicolumn{3}{|c|}{$\begin{array}{l}\text { Heat treated at } 850^{\circ} \mathrm{C} \text { or at } 750^{\circ} \mathrm{C} \text { for } 20 \text { hours after premelt at } 1200^{\circ} \mathrm{C} \text { : } \\
\text { Optical microscopy examination }\end{array}$} & Crystal-free glass \\
\hline \multicolumn{3}{|c|}{$\begin{array}{l}\text { Subjected to LAW Centerline Canister Cooling: } \\
\text { Optical microscopy examination }\end{array}$} & Not available \\
\hline \multicolumn{4}{|c|}{ Viscosity (Poise) } \\
\hline \multicolumn{3}{|c|}{$950^{\circ} \mathrm{C}$} & 1018 \\
\hline \multicolumn{3}{|c|}{$1000^{\circ} \mathrm{C}$} & 448 \\
\hline \multicolumn{3}{|c|}{$1050^{\circ} \mathrm{C}$} & 219 \\
\hline \multicolumn{3}{|c|}{$1100^{\circ} \mathrm{C}$} & 117 \\
\hline \multicolumn{3}{|c|}{$1150^{\circ} \mathrm{C}$} & 67 \\
\hline \multicolumn{3}{|c|}{$1200^{\circ} \mathrm{C}$} & 41 \\
\hline \multicolumn{3}{|c|}{$1250^{\circ} \mathrm{C}$} & 26 \\
\hline \multicolumn{4}{|c|}{ Electrical Conductivity $(\mathrm{S} / \mathrm{cm})$} \\
\hline \multicolumn{3}{|c|}{$950^{\circ} \mathrm{C}$} & 0.101 \\
\hline \multicolumn{3}{|c|}{$1000^{\circ} \mathrm{C}$} & 0.135 \\
\hline \multicolumn{3}{|c|}{$1050^{\circ} \mathrm{C}$} & 0.175 \\
\hline \multicolumn{3}{|c|}{$1100^{\circ} \mathrm{C}$} & 0.223 \\
\hline \multicolumn{3}{|c|}{$1150^{\circ} \mathrm{C}$} & 0.280 \\
\hline \multicolumn{3}{|c|}{$1200^{\circ} \mathrm{C}$} & 0.346 \\
\hline \multicolumn{3}{|c|}{$1250^{\circ} \mathrm{C}$} & 0.421 \\
\hline \multicolumn{3}{|c|}{$\begin{aligned} \mathrm{B}\left(\mathrm{g} / \mathrm{m}^{2}\right) \\
\mathrm{Na}\left(\mathrm{g} / \mathrm{m}^{2}\right) \\
\mathrm{Si}\left(\mathrm{g} / \mathrm{m}^{2}\right)\end{aligned}$} & $\begin{array}{l}0.275 \\
0.313 \\
0.120\end{array}$ \\
\hline \multicolumn{3}{|c|}{ VHT (g/m²/day) (specification: $<50 \mathrm{~g} / \mathrm{m}^{2} /$ day) } & 12 \\
\hline \multicolumn{3}{|c|}{$\mathrm{T}_{\mathrm{G}}$ (onset) } & $464^{\circ} \mathrm{C}$ \\
\hline
\end{tabular}




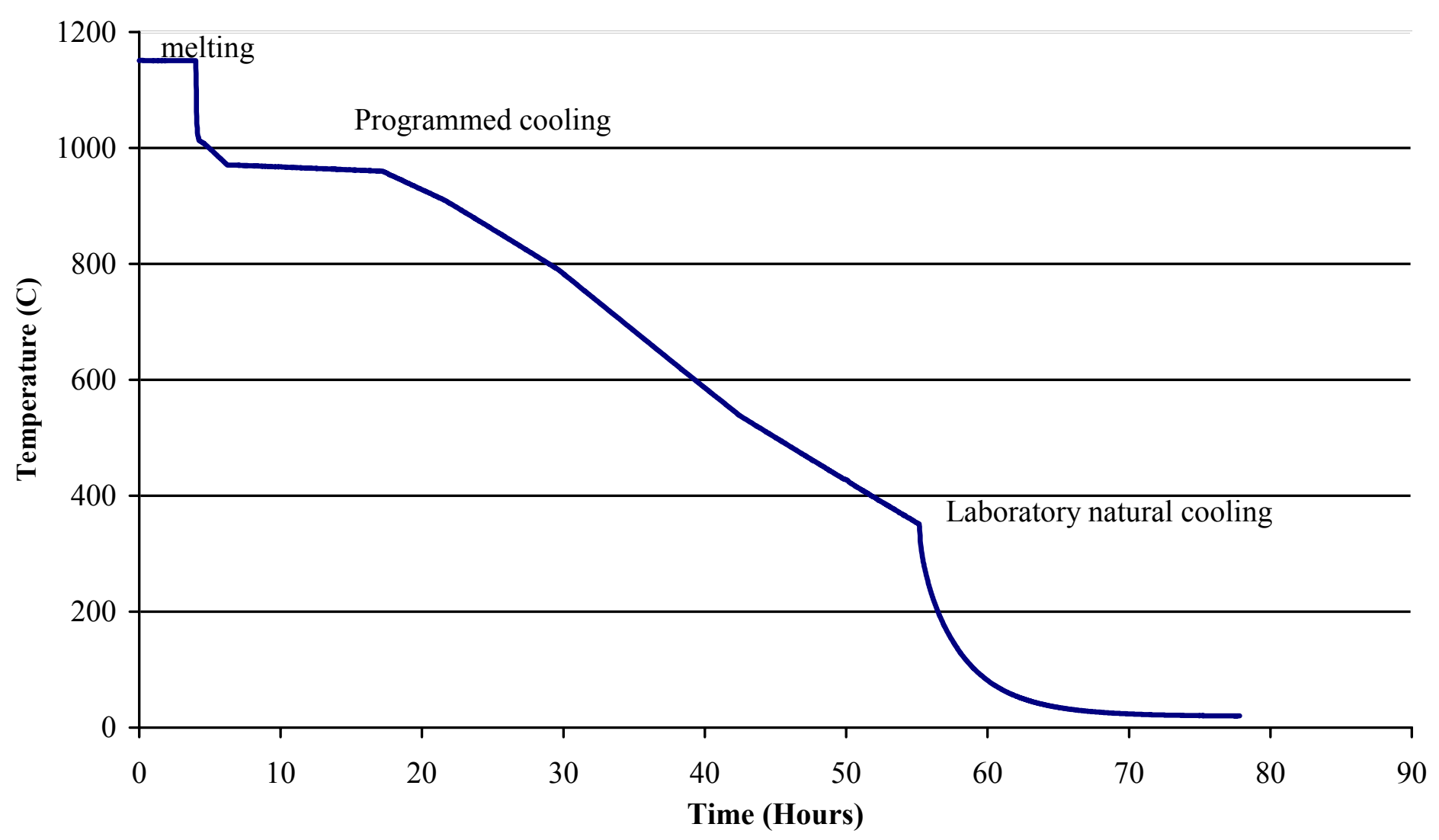

Figure 1. Centerline canister cooling curve used for heat treatment of LAWA44CC and LAWA88CC. 


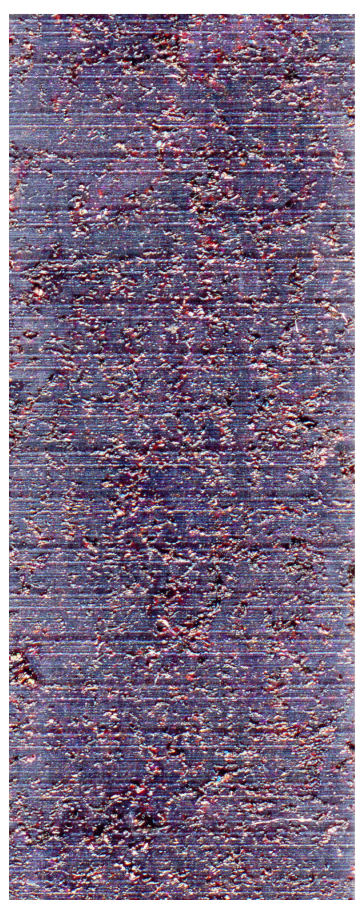

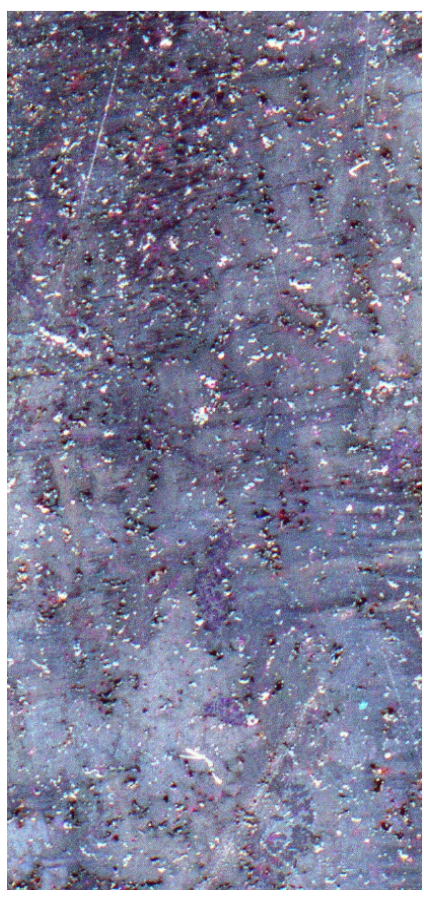

WVDP Ref. 6

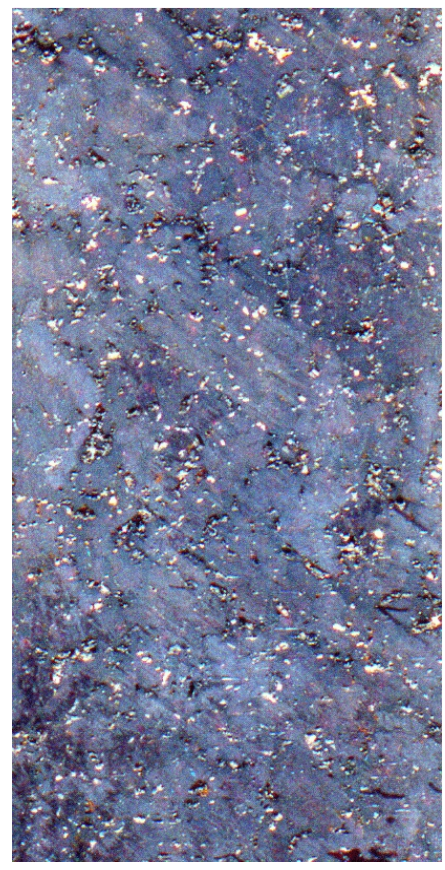

LAWA44

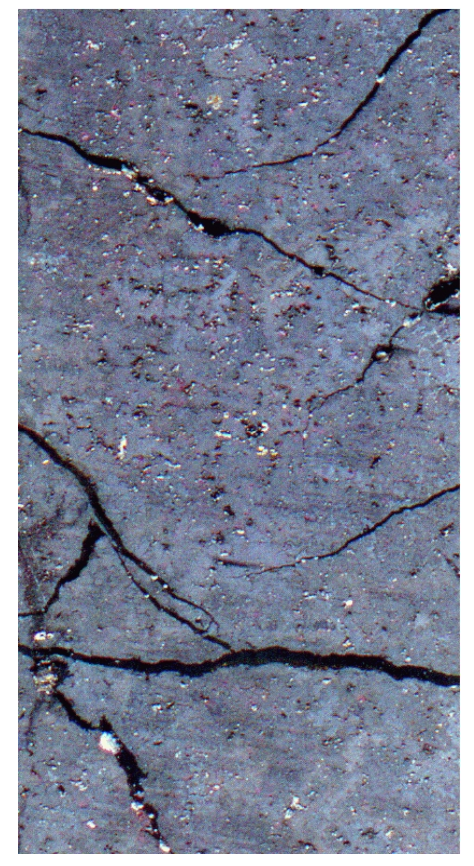

LAWB64

Figure 2. Optical images of K-3 refractory coupons before corrosion testing ("unreacted") and after six-day corrosion testing in the indicated glass formulation [29] illustrating the "cracking" discussed in the text. 


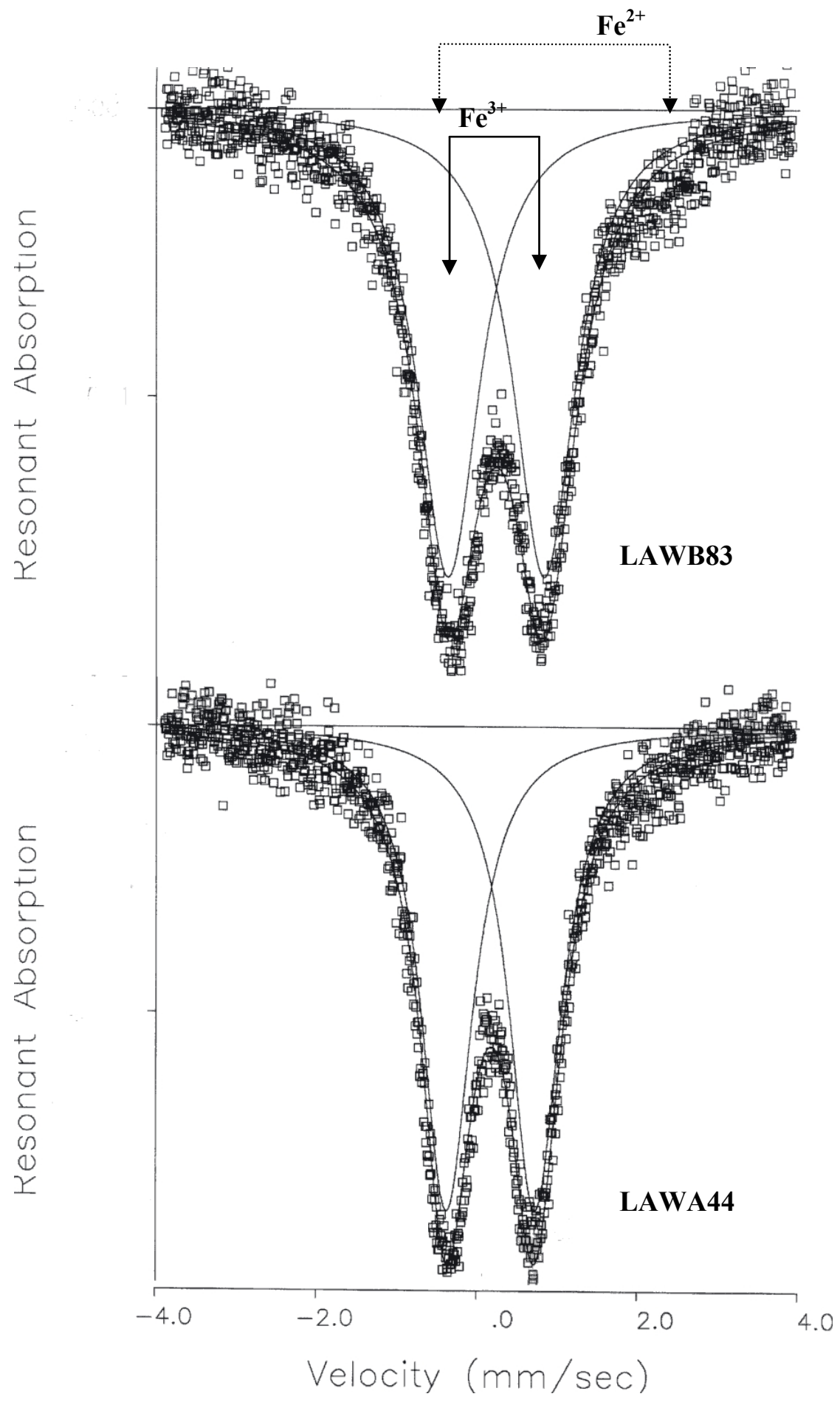

Figure 3. Mossbauer spectra for LAWA44 and LAWB83 glasses showing all iron as $\mathrm{Fe}^{3+}$. No reduction was observed $\left(\mathrm{Fe}^{2+}\right.$ would be evidenced by a peak at $\approx 2.3 \mathrm{~mm} / \mathrm{sec}$ ). 


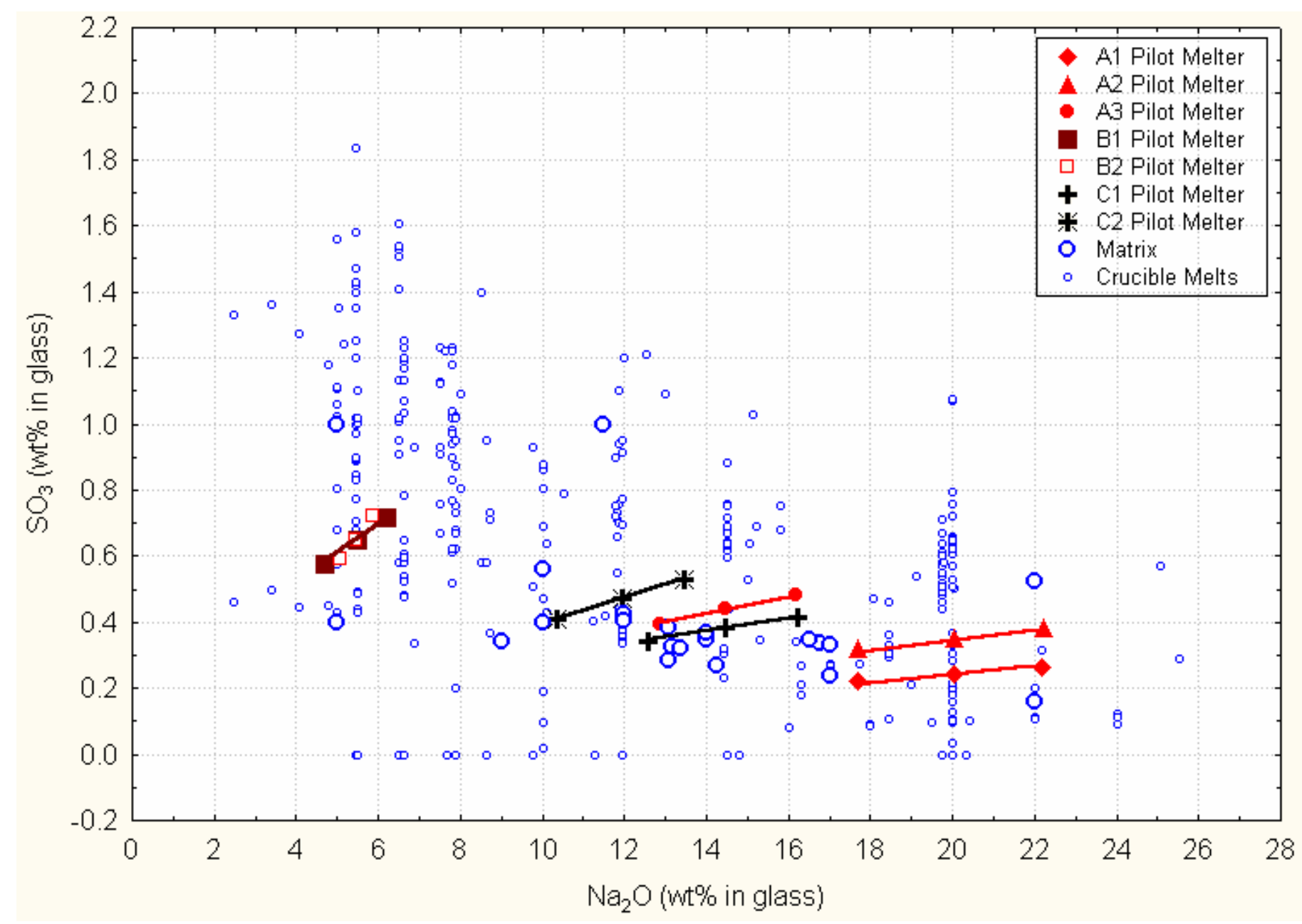

Figure 4. Sulfate content measured in crucible melt glasses from this work and previous work [1, 2] as a function of their sodium content. Also included are formulations tested on the DM100 and LAW Pilot Melter and compositions from the ILAW test matrix [11]. Note that only a portion of the sulfate solubility limit is practically achievable in a melter due to kinetic effects $[14,15,37,38]$. 


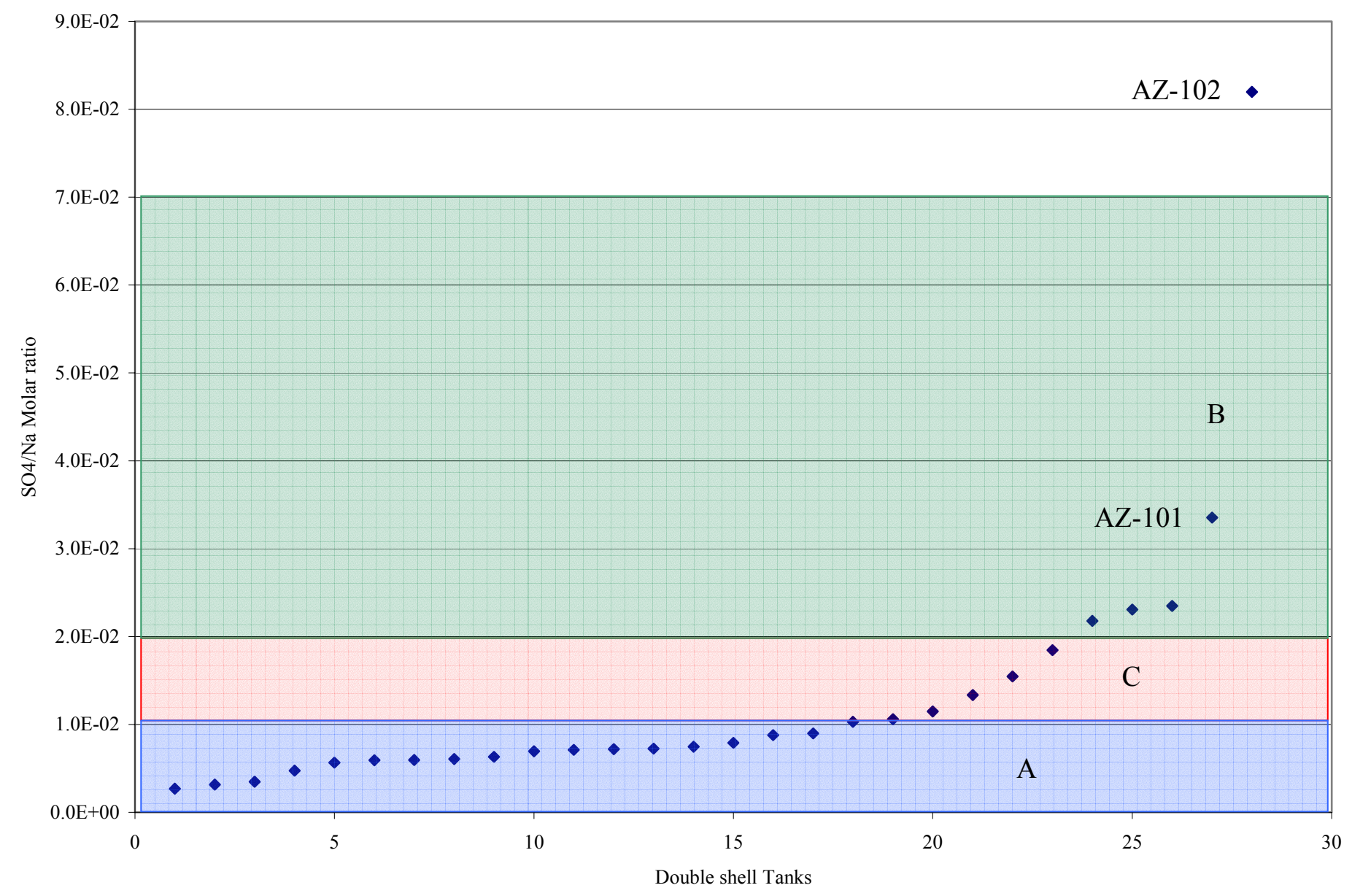

Figure 5. Plot of the sulfate to sodium molar ratio estimated for the LAW feeds for all 28 double-shell tanks, as reported in TFCOUP Rev. 3A [7] with the assumption that $50 \%$ of sodium and $75 \%$ of the sulfate in the solid fraction would be returned to the liquid feed. The data points are compared to the limits defining the LAW Envelopes. 


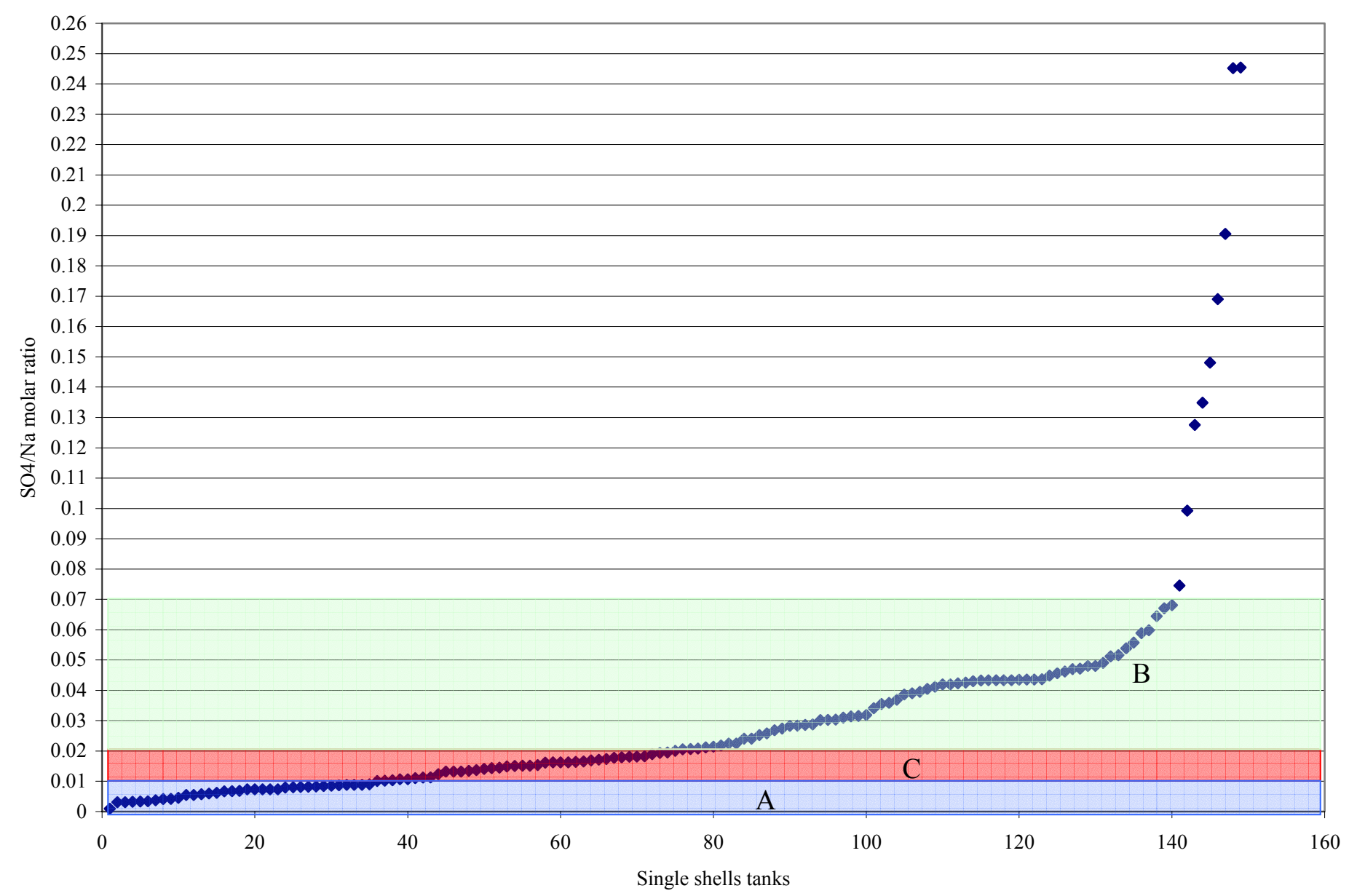

Figure 6. Plot of the sulfate to sodium molar ratio estimated for the LAW feeds for all 149 of the single-shell tanks, as reported in TFCOUP Rev. 3A [7] with the assumption that $50 \%$ of sodium and $75 \%$ of the sulfate in the solid fraction would be returned to the liquid feed. The data points are compared to the limits defining the LAW Envelopes. 
Appendix A1: Estimate of the Sulfate to Sodium Ratio Expected in the LAW Feed Delivered for 28 Double Shell Tanks Per TFCOUP Rev. 3A.

\begin{tabular}{|c|c|c|c|c|c|c|c|c|c|c|c|}
\hline & \multirow{2}{*}{\multicolumn{2}{|c|}{ Liquid fraction }} & \multirow{2}{*}{\multicolumn{2}{|c|}{ Solid fraction }} & \multirow{2}{*}{\multicolumn{2}{|c|}{ Liquid fraction }} & \multirow{2}{*}{\multicolumn{2}{|c|}{ Solid fraction }} & \multirow{3}{*}{$\begin{array}{c}\text { Total Na } \\
\text { Estimated* }\end{array}$} & \multirow{3}{*}{$\begin{array}{c}\text { Total } \mathrm{SO}_{4} \\
\text { Estimated* }\end{array}$} & \\
\hline & & & & & & & & & & & \multirow{3}{*}{$\begin{array}{c}\mathrm{SO}_{4} / \mathrm{Na} \\
\text { Estimated* }\end{array}$} \\
\hline & \multirow{2}{*}{$\begin{array}{l}\mathrm{Na} \\
\mathrm{Kg}\end{array}$} & \multirow{2}{*}{$\frac{\mathrm{SO}_{4}}{\mathrm{~kg}}$} & \multirow{2}{*}{$\begin{array}{l}\mathrm{Na} \\
\mathrm{kg}\end{array}$} & \multirow{2}{*}{$\begin{array}{c}\mathrm{SO}_{4} \\
\mathrm{~kg}\end{array}$} & \multirow{2}{*}{$\begin{array}{l}\mathrm{Na} \\
\mathrm{mol} .\end{array}$} & \multirow{2}{*}{$\begin{array}{l}\mathrm{SO}_{4} \\
\mathrm{~mol} .\end{array}$} & \multirow{2}{*}{$\begin{array}{l}\mathrm{Na} \\
\mathrm{mol} .\end{array}$} & \multirow{2}{*}{$\begin{array}{l}\mathrm{SO}_{4} \\
\mathrm{~mol} .\end{array}$} & & & \\
\hline $\begin{array}{l}\text { Appenaix } \\
\text { Species } \\
\text { Units }\end{array}$ & & & & & & & & & mol. & mol. & \\
\hline AN-101 & $7.35 \mathrm{E}+04$ & $1.07 \mathrm{E}+03$ & $0.00 \mathrm{E}+00$ & $0.00 \mathrm{E}+00$ & $\begin{array}{l}3.20 \mathrm{E}+00 \\
3.61 \mathrm{E}+01\end{array}$ & $\begin{array}{l}1.12 \mathrm{E}-02 \\
5.82 \mathrm{E}-01 \\
\end{array}$ & $0.00 \mathrm{E}+00$ & $\begin{array}{r}0.00 \mathrm{E}+00 \\
1.60 \mathrm{E}-01\end{array}$ & $3.20 \mathrm{E}+00$ & $\begin{array}{l}1.12 \mathrm{E}-02 \\
7.02 \mathrm{E}-01\end{array}$ & $3.50 \mathrm{E}-03$ \\
\hline AN-103 & $5.35 \mathrm{E}+05$ & $2.73 \mathrm{E}+03$ & $5.69 \mathrm{E}+05$ & $8.65 \mathrm{E}+03$ & $2.33 \mathrm{E}+01$ & $2.84 \mathrm{E}-02$ & $2.47 \mathrm{E}+01$ & $9.01 \mathrm{E}-02$ & $3.57 \mathrm{E}+01$ & $9.59 \mathrm{E}-02$ & $2.69 \mathrm{E}-03$ \\
\hline AN-104 & $5.82 \mathrm{E}+05$ & $8.05 \mathrm{E}+03$ & $4.89 \mathrm{E}+05$ & $3.07 \mathrm{E}+04$ & $2.53 \mathrm{E}+01$ & $8.38 \mathrm{E}-02$ & $2.13 \mathrm{E}+01$ & $3.20 \mathrm{E}-01$ & $3.60 \mathrm{E}+01$ & $3.24 \mathrm{E}-01$ & $9.00 \mathrm{E}-03$ \\
\hline AN-105 & $5.90 \mathrm{E}+05$ & $6.84 \mathrm{E}+03$ & $5.08 \mathrm{E}+05$ & $2.07 \mathrm{E}+04$ & $2.57 \mathrm{E}+01$ & 7.12E-02 & $2.21 \mathrm{E}+01$ & $2.15 \mathrm{E}-01$ & $3.67 \mathrm{E}+01$ & $2.33 \mathrm{E}-01$ & $6.33 \mathrm{E}-03$ \\
\hline AP-101 & $5.45 \mathrm{E}+05$ & $1.70 \mathrm{E}+04$ & $0.00 \mathrm{E}+00$ & $0.00 \mathrm{E}+00$ & $2.37 \mathrm{E}+01$ & $1.77 \mathrm{E}-01$ & $0.00 \mathrm{E}+00$ & $0.00 \mathrm{E}+00$ & $2.37 \mathrm{E}+01$ & $1.77 \mathrm{E}-01$ & 7.46E-03 \\
\hline AP-102 & $4.23 \mathrm{E}+05$ & $1.88 \mathrm{E}+04$ & $0.00 \mathrm{E}+00$ & $0.00 \mathrm{E}+00$ & $1.84 \mathrm{E}+01$ & $1.95 \mathrm{E}-01$ & $0.00 \mathrm{E}+00$ & $0.00 \mathrm{E}+00$ & $1.84 \mathrm{E}+01$ & $1.95 \mathrm{E}-01$ & $1.06 \mathrm{E}-02$ \\
\hline AP-103 & $1.94 \mathrm{E}+05$ & $4.82 \mathrm{E}+03$ & $0.00 \mathrm{E}+00$ & $0.00 \mathrm{E}+00$ & $8.43 \mathrm{E}+00$ & $5.02 \mathrm{E}-02$ & $0.00 \mathrm{E}+00$ & $0.00 \mathrm{E}+00$ & $8.43 \mathrm{E}+00$ & $5.02 \mathrm{E}-02$ & $5.95 \mathrm{E}-03$ \\
\hline AP-104 & $5.04 \mathrm{E}+05$ & $1.47 \mathrm{E}+04$ & $0.00 \mathrm{E}+00$ & $0.00 \mathrm{E}+00$ & $2.19 \mathrm{E}+01$ & $1.53 \mathrm{E}-01$ & $0.00 \mathrm{E}+00$ & $0.00 \mathrm{E}+00$ & $2.19 \mathrm{E}+01$ & $1.53 \mathrm{E}-01$ & $6.96 \mathrm{E}-03$ \\
\hline AP-105 & $7.51 \mathrm{E}+05$ & $2.69 \mathrm{E}+04$ & $6.71 \mathrm{E}+04$ & $2.63 \mathrm{E}+03$ & $3.27 \mathrm{E}+01$ & $2.80 \mathrm{E}-01$ & $2.92 \mathrm{E}+00$ & $2.74 \mathrm{E}-02$ & $3.41 \mathrm{E}+01$ & 3.00E-01 & $8.80 \mathrm{E}-03$ \\
\hline AP-106 & $2.90 \mathrm{E}+05$ & $6.85 \mathrm{E}+03$ & $0.00 \mathrm{E}+00$ & $0.00 \mathrm{E}+00$ & $1.26 \mathrm{E}+01$ & $7.13 \mathrm{E}-02$ & $0.00 \mathrm{E}+00$ & $0.00 \mathrm{E}+00$ & $1.26 \mathrm{E}+01$ & 7.13E-02 & $5.66 \mathrm{E}-03$ \\
\hline AW-103 & $1.39 \mathrm{E}+04$ & $3.79 \mathrm{E}+02$ & $2.83 \mathrm{E}+05$ & $3.60 \mathrm{E}+03$ & $6.03 \mathrm{E}-01$ & $3.95 \mathrm{E}-03$ & $1.23 \mathrm{E}+01$ & $3.75 \mathrm{E}-02$ & $6.75 \mathrm{E}+00$ & $3.21 \mathrm{E}-02$ & $4.75 \mathrm{E}-03$ \\
\hline AW-104 & $5.90 \mathrm{E}+04$ & $2.22 \mathrm{E}+03$ & $2.25 \mathrm{E}+05$ & $1.91 \mathrm{E}+04$ & $2.56 \mathrm{E}+00$ & $2.31 \mathrm{E}-02$ & $9.81 \mathrm{E}+00$ & $1.99 \mathrm{E}-01$ & $7.47 \mathrm{E}+00$ & $1.72 \mathrm{E}-01$ & $2.31 \mathrm{E}-02$ \\
\hline AW-105 & $1.01 \mathrm{E}+04$ & $2.04 \mathrm{E}+02$ & $1.29 \mathrm{E}+05$ & $2.68 \mathrm{E}+03$ & $4.39 \mathrm{E}-01$ & $2.12 \mathrm{E}-03$ & $5.61 \mathrm{E}+00$ & $2.79 \mathrm{E}-02$ & $3.24 \mathrm{E}+00$ & $2.30 \mathrm{E}-02$ & $7.11 \mathrm{E}-03$ \\
\hline AW-106 & $3.36 \mathrm{E}+05$ & $9.76 \mathrm{E}+03$ & $2.50 \mathrm{E}+05$ & $2.13 \mathrm{E}+04$ & $1.46 \mathrm{E}+01$ & $1.02 \mathrm{E}-01$ & $1.09 \mathrm{E}+01$ & $2.22 \mathrm{E}-01$ & $2.01 \mathrm{E}+01$ & $2.68 \mathrm{E}-01$ & $1.34 \mathrm{E}-02$ \\
\hline AY-101 & $9.03 \mathrm{E}+03$ & $1.02 \mathrm{E}+03$ & $3.81 \mathrm{E}+04$ & $2.05 \mathrm{E}+03$ & $3.93 \mathrm{E}-01$ & $1.06 \mathrm{E}-02$ & $1.66 \mathrm{E}+00$ & $2.13 \mathrm{E}-02$ & $1.22 \mathrm{E}+00$ & $2.66 \mathrm{E}-02$ & $2.18 \mathrm{E}-02$ \\
\hline AY-102 & $8.85 \mathrm{E}+04$ & $3.80 \mathrm{E}+03$ & $6.45 \mathrm{E}+04$ & $1.86 \mathrm{E}+03$ & $3.85 \mathrm{E}+00$ & $3.96 \mathrm{E}-02$ & $2.81 \mathrm{E}+00$ & $1.94 \mathrm{E}-02$ & $5.25 \mathrm{E}+00$ & $5.41 \mathrm{E}-02$ & $1.03 \mathrm{E}-02$ \\
\hline AZ-101 & $3.35 \mathrm{E}+05$ & $4.53 \mathrm{E}+04$ & $2.89 \mathrm{E}+04$ & $4.83 \mathrm{E}+03$ & $1.46 \mathrm{E}+01$ & 4.72E-01 & $1.26 \mathrm{E}+00$ & 5.03E-02 & $1.52 \mathrm{E}+01$ & $5.10 \mathrm{E}-01$ & $3.35 \mathrm{E}-02$ \\
\hline AZ-102 & $1.75 \mathrm{E}+05$ & $5.81 \mathrm{E}+04$ & $2.53 \mathrm{E}+04$ & $8.05 \mathrm{E}+03$ & $7.60 \mathrm{E}+00$ & $6.05 \mathrm{E}-01$ & $1.10 \mathrm{E}+00$ & $8.38 \mathrm{E}-02$ & $8.15 \mathrm{E}+00$ & $6.68 \mathrm{E}-01$ & $8.20 \mathrm{E}-02$ \\
\hline SY-101 & $5.70 \mathrm{E}+05$ & $1.38 \mathrm{E}+04$ & $9.05 \mathrm{E}+04$ & $2.03 \mathrm{E}+03$ & $2.48 \mathrm{E}+01$ & $1.43 \mathrm{E}-01$ & $3.94 \mathrm{E}+00$ & $2.11 \mathrm{E}-02$ & $2.68 \mathrm{E}+01$ & $1.59 \mathrm{E}-01$ & $5.94 \mathrm{E}-03$ \\
\hline SY-102 & $2.89 \mathrm{E}+05$ & $8.55 \mathrm{E}+03$ & $3.08 \mathrm{E}+04$ & $2.01 \mathrm{E}+03$ & $1.26 \mathrm{E}+01$ & $8.90 \mathrm{E}-02$ & $1.34 \mathrm{E}+00$ & $2.10 \mathrm{E}-02$ & $1.32 \mathrm{E}+01$ & 1.05E-01 & $7.91 \mathrm{E}-03$ \\
\hline SY-103 & $2.86 \mathrm{E}+05$ & $0.00 \mathrm{E}+00$ & $4.33 \mathrm{E}+05$ & $1.70 \mathrm{E}+04$ & $1.24 \mathrm{E}+01$ & $0.00 \mathrm{E}+00$ & $1.88 \mathrm{E}+01$ & $1.77 \mathrm{E}-01$ & $2.19 \mathrm{E}+01$ & $1.33 \mathrm{E}-01$ & $6.08 \mathrm{E}-03$ \\
\hline
\end{tabular}

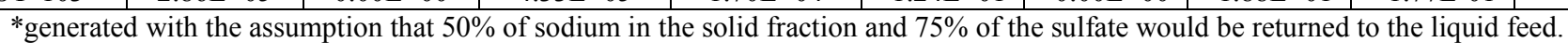




\begin{tabular}{|c|c|c|c|c|c|c|c|c|c|c|c|}
\hline \multirow[b]{2}{*}{ Species } & \multicolumn{2}{|c|}{ Liquid fraction } & \multicolumn{2}{|c|}{ Solid fraction } & \multicolumn{2}{|c|}{ Liquid fraction } & \multicolumn{2}{|c|}{ Solid fraction } & \multirow{2}{*}{$\begin{array}{c}\text { Total Na } \\
\text { Estimated* }\end{array}$} & \multirow{2}{*}{$\begin{array}{c}\text { Total } \mathrm{SO}_{4} \\
\text { Estimated* }\end{array}$} & \multirow{3}{*}{$\begin{array}{c}\mathrm{SO}_{4} / \mathrm{Na} \\
\text { Estimated* }\end{array}$} \\
\hline & $\mathrm{Na}$ & $\mathrm{SO}_{4}$ & $\mathrm{Na}$ & $\mathrm{SO}_{4}$ & $\mathrm{Na}$ & $\mathrm{SO}_{4}$ & $\mathrm{Na}$ & $\mathrm{SO}_{4}$ & & & \\
\hline Units & $\mathrm{Kg}$ & $\mathrm{kg}$ & $\mathrm{kg}$ & $\mathrm{kg}$ & mol. & mol. & mol. & mol. & mol. & mol. & \\
\hline A-101 & $6.38 \mathrm{E}+05$ & $3.22 \mathrm{E}+03$ & $3.75 \mathrm{E}+05$ & $5.77 \mathrm{E}+04$ & $2.78 \mathrm{E}+01$ & $3.35 \mathrm{E}-02$ & $1.63 \mathrm{E}+01$ & $6.01 \mathrm{E}-01$ & $3.59 \mathrm{E}+01$ & 4.84E-01 & $1.35 \mathrm{E}-02$ \\
\hline A-102 & $0.00 \mathrm{E}+00$ & $0.00 \mathrm{E}+00$ & $4.03 \mathrm{E}+04$ & $1.14 \mathrm{E}+03$ & $0.00 \mathrm{E}+00$ & $0.00 \mathrm{E}+00$ & $1.75 \mathrm{E}+00$ & $1.19 \mathrm{E}-02$ & $8.76 \mathrm{E}-01$ & $8.90 \mathrm{E}-03$ & $1.02 \mathrm{E}-02$ \\
\hline A-103 & $1.67 \mathrm{E}+04$ & $0.00 \mathrm{E}+00$ & $3.91 \mathrm{E}+05$ & $3.40 \mathrm{E}+04$ & $7.26 \mathrm{E}-01$ & $0.00 \mathrm{E}+00$ & $1.70 \mathrm{E}+01$ & $3.54 \mathrm{E}-01$ & $9.23 \mathrm{E}+00$ & $2.65 \mathrm{E}-01$ & $2.88 \mathrm{E}-02$ \\
\hline A-104 & $0.00 \mathrm{E}+00$ & $0.00 \mathrm{E}+00$ & $1.34 \mathrm{E}+04$ & $6.80 \mathrm{E}+02$ & $0.00 \mathrm{E}+00$ & $0.00 \mathrm{E}+00$ & $5.83 \mathrm{E}-01$ & $7.08 \mathrm{E}-03$ & 2.91E-01 & $5.31 \mathrm{E}-03$ & $1.82 \mathrm{E}-02$ \\
\hline A-105 & $0.00 \mathrm{E}+00$ & $0.00 \mathrm{E}+00$ & $5.50 \mathrm{E}+04$ & $7.36 \mathrm{E}+03$ & $0.00 \mathrm{E}+00$ & $0.00 \mathrm{E}+00$ & $2.39 \mathrm{E}+00$ & $7.66 \mathrm{E}-02$ & $1.20 \mathrm{E}+00$ & $5.75 \mathrm{E}-02$ & $4.80 \mathrm{E}-02$ \\
\hline A-106 & $0.00 \mathrm{E}+00$ & $0.00 \mathrm{E}+00$ & $8.94 \mathrm{E}+04$ & $3.29 \mathrm{E}+03$ & $0.00 \mathrm{E}+00$ & $0.00 \mathrm{E}+00$ & $3.89 \mathrm{E}+00$ & $3.42 \mathrm{E}-02$ & $1.94 \mathrm{E}+00$ & $2.57 \mathrm{E}-02$ & $1.32 \mathrm{E}-02$ \\
\hline AX-101 & $5.01 \mathrm{E}+05$ & $2.74 \mathrm{E}+03$ & $3.26 \mathrm{E}+05$ & $5.23 \mathrm{E}+04$ & $2.18 \mathrm{E}+01$ & $2.85 \mathrm{E}-02$ & $1.42 \mathrm{E}+01$ & $5.44 \mathrm{E}-01$ & $2.89 \mathrm{E}+01$ & 4.37E-01 & $1.51 \mathrm{E}-02$ \\
\hline AX-102 & $0.00 \mathrm{E}+00$ & $0.00 \mathrm{E}+00$ & $2.87 \mathrm{E}+04$ & $6.38 \mathrm{E}+02$ & $0.00 \mathrm{E}+00$ & $0.00 \mathrm{E}+00$ & $1.25 \mathrm{E}+00$ & $6.64 \mathrm{E}-03$ & $6.24 \mathrm{E}-01$ & $4.98 \mathrm{E}-03$ & $7.98 \mathrm{E}-03$ \\
\hline AX-103 & $0.00 \mathrm{E}+00$ & $0.00 \mathrm{E}+00$ & $1.27 \mathrm{E}+05$ & $1.07 \mathrm{E}+04$ & $0.00 \mathrm{E}+00$ & $0.00 \mathrm{E}+00$ & $5.52 \mathrm{E}+00$ & $1.11 \mathrm{E}-01$ & $2.76 \mathrm{E}+00$ & $8.35 \mathrm{E}-02$ & $3.02 \mathrm{E}-02$ \\
\hline AX-104 & $0.00 \mathrm{E}+00$ & $0.00 \mathrm{E}+00$ & $2.20 \mathrm{E}+03$ & $2.42 \mathrm{E}+02$ & $0.00 \mathrm{E}+00$ & $0.00 \mathrm{E}+00$ & $9.57 \mathrm{E}-02$ & $2.52 \mathrm{E}-03$ & $4.78 \mathrm{E}-02$ & $1.89 \mathrm{E}-03$ & $3.95 \mathrm{E}-02$ \\
\hline B-101 & $0.00 \mathrm{E}+00$ & $0.00 \mathrm{E}+00$ & $1.57 \mathrm{E}+05$ & $2.98 \mathrm{E}+04$ & $0.00 \mathrm{E}+00$ & $0.00 \mathrm{E}+00$ & $6.83 \mathrm{E}+00$ & $3.10 \mathrm{E}-01$ & $3.41 \mathrm{E}+00$ & $2.33 \mathrm{E}-01$ & $6.81 \mathrm{E}-02$ \\
\hline B-102 & $0.00 \mathrm{E}+00$ & $0.00 \mathrm{E}+00$ & $5.47 \mathrm{E}+04$ & $3.74 \mathrm{E}+04$ & $0.00 \mathrm{E}+00$ & $0.00 \mathrm{E}+00$ & $2.38 \mathrm{E}+00$ & $3.89 \mathrm{E}-01$ & $1.19 \mathrm{E}+00$ & $2.92 \mathrm{E}-01$ & $2.45 \mathrm{E}-01$ \\
\hline B-104 & $0.00 \mathrm{E}+00$ & $0.00 \mathrm{E}+00$ & $2.27 \mathrm{E}+05$ & $4.24 \mathrm{E}+04$ & $0.00 \mathrm{E}+00$ & $0.00 \mathrm{E}+00$ & $9.87 \mathrm{E}+00$ & $4.41 \mathrm{E}-01$ & $4.94 \mathrm{E}+00$ & $3.31 \mathrm{E}-01$ & $6.71 \mathrm{E}-02$ \\
\hline B-105 & $1.27 \mathrm{E}+04$ & $2.30 \mathrm{E}+03$ & $1.60 \mathrm{E}+05$ & $6.28 \mathrm{E}+04$ & $5.52 \mathrm{E}-01$ & $2.39 \mathrm{E}-02$ & $6.96 \mathrm{E}+00$ & $6.54 \mathrm{E}-01$ & $4.03 \mathrm{E}+00$ & $5.14 \mathrm{E}-01$ & $1.28 \mathrm{E}-01$ \\
\hline B-106 & $0.00 \mathrm{E}+00$ & $0.00 \mathrm{E}+00$ & $6.73 \mathrm{E}+04$ & $1.01 \mathrm{E}+04$ & $0.00 \mathrm{E}+00$ & $0.00 \mathrm{E}+00$ & $2.93 \mathrm{E}+00$ & $1.05 \mathrm{E}-01$ & $1.46 \mathrm{E}+00$ & $7.89 \mathrm{E}-02$ & $5.39 \mathrm{E}-02$ \\
\hline B-107 & $0.00 \mathrm{E}+00$ & $0.00 \mathrm{E}+00$ & $1.63 \mathrm{E}+05$ & $8.65 \mathrm{E}+04$ & $0.00 \mathrm{E}+00$ & $0.00 \mathrm{E}+00$ & $7.09 \mathrm{E}+00$ & $9.00 \mathrm{E}-01$ & $3.55 \mathrm{E}+00$ & $6.75 \mathrm{E}-01$ & $1.91 \mathrm{E}-01$ \\
\hline B-108 & $0.00 \mathrm{E}+00$ & $0.00 \mathrm{E}+00$ & $9.19 \mathrm{E}+04$ & $3.79 \mathrm{E}+04$ & $0.00 \mathrm{E}+00$ & $0.00 \mathrm{E}+00$ & $4.00 \mathrm{E}+00$ & $3.95 \mathrm{E}-01$ & $2.00 \mathrm{E}+00$ & $2.96 \mathrm{E}-01$ & $1.48 \mathrm{E}-01$ \\
\hline B-109 & $0.00 \mathrm{E}+00$ & $0.00 \mathrm{E}+00$ & $1.79 \mathrm{E}+05$ & $8.43 \mathrm{E}+04$ & $0.00 \mathrm{E}+00$ & $0.00 \mathrm{E}+00$ & $7.79 \mathrm{E}+00$ & $8.78 \mathrm{E}-01$ & $3.89 \mathrm{E}+00$ & $6.58 \mathrm{E}-01$ & $1.69 \mathrm{E}-01$ \\
\hline B-110 & $0.00 \mathrm{E}+00$ & $0.00 \mathrm{E}+00$ & $1.23 \mathrm{E}+05$ & $1.45 \mathrm{E}+04$ & $0.00 \mathrm{E}+00$ & $0.00 \mathrm{E}+00$ & $5.35 \mathrm{E}+00$ & $1.51 \mathrm{E}-01$ & $2.68 \mathrm{E}+00$ & $1.13 \mathrm{E}-01$ & $4.23 \mathrm{E}-02$ \\
\hline B-111 & $0.00 \mathrm{E}+00$ & $0.00 \mathrm{E}+00$ & $1.02 \mathrm{E}+05$ & $1.23 \mathrm{E}+04$ & $0.00 \mathrm{E}+00$ & $0.00 \mathrm{E}+00$ & $4.44 \mathrm{E}+00$ & $1.28 \mathrm{E}-01$ & $2.22 \mathrm{E}+00$ & $9.60 \mathrm{E}-02$ & $4.33 \mathrm{E}-02$ \\
\hline B-112 & $1.40 \mathrm{E}+03$ & $1.67 \mathrm{E}+02$ & $1.40 \mathrm{E}+04$ & $1.67 \mathrm{E}+03$ & $6.09 \mathrm{E}-02$ & $1.74 \mathrm{E}-03$ & $6.09 \mathrm{E}-01$ & $1.74 \mathrm{E}-02$ & $3.65 \mathrm{E}-01$ & $1.48 \mathrm{E}-02$ & 4.04E-02 \\
\hline B-201 & $0.00 \mathrm{E}+00$ & $0.00 \mathrm{E}+00$ & $5.24 \mathrm{E}+03$ & $4.78 \mathrm{E}+01$ & $0.00 \mathrm{E}+00$ & $0.00 \mathrm{E}+00$ & $2.28 \mathrm{E}-01$ & $4.98 \mathrm{E}-04$ & $1.14 \mathrm{E}-01$ & $3.73 \mathrm{E}-04$ & $3.27 \mathrm{E}-03$ \\
\hline B-202 & $0.00 \mathrm{E}+00$ & $0.00 \mathrm{E}+00$ & $4.58 \mathrm{E}+03$ & $1.90 \mathrm{E}+02$ & $0.00 \mathrm{E}+00$ & $0.00 \mathrm{E}+00$ & $1.99 \mathrm{E}-01$ & $1.98 \mathrm{E}-03$ & $9.96 \mathrm{E}-02$ & $1.48 \mathrm{E}-03$ & $1.49 \mathrm{E}-02$ \\
\hline B-203 & $0.00 \mathrm{E}+00$ & $0.00 \mathrm{E}+00$ & $6.66 \mathrm{E}+03$ & $1.61 \mathrm{E}+02$ & $0.00 \mathrm{E}+00$ & $0.00 \mathrm{E}+00$ & $2.90 \mathrm{E}-01$ & $1.68 \mathrm{E}-03$ & $1.45 \mathrm{E}-01$ & $1.26 \mathrm{E}-03$ & $8.68 \mathrm{E}-03$ \\
\hline B-204 & $1.11 \mathrm{E}+02$ & $3.12 \mathrm{E}+00$ & $5.77 \mathrm{E}+03$ & $1.43 \mathrm{E}+02$ & $4.83 \mathrm{E}-03$ & $3.25 \mathrm{E}-05$ & $2.51 \mathrm{E}-01$ & $1.49 \mathrm{E}-03$ & $1.30 \mathrm{E}-01$ & $1.15 \mathrm{E}-03$ & $8.82 \mathrm{E}-03$ \\
\hline BX-101 & $5.32 \mathrm{E}+02$ & $1.48 \mathrm{E}+01$ & $2.00 \mathrm{E}+04$ & $4.81 \mathrm{E}+02$ & $2.31 \mathrm{E}-02$ & $1.54 \mathrm{E}-04$ & $8.70 \mathrm{E}-01$ & $5.01 \mathrm{E}-03$ & $4.58 \mathrm{E}-01$ & $3.91 \mathrm{E}-03$ & $8.53 \mathrm{E}-03$ \\
\hline
\end{tabular}

*generated with the assumption that $50 \%$ of sodium in the solid fraction and $75 \%$ of the sulfate would be returned to the liquid feed. 
Appendix A2: Estimate of the Sulfate to Sodium Ratio Expected in the LAW Feed Delivered for 149 Single Shell Tanks Per TFCOUP Rev. 3A (cont'd).

\begin{tabular}{|c|c|c|c|c|c|c|c|c|c|c|c|}
\hline \multirow[b]{2}{*}{ Species } & \multicolumn{2}{|c|}{ Liquid fraction } & \multicolumn{2}{|c|}{ Solid fraction } & \multicolumn{2}{|c|}{ Liquid fraction } & \multicolumn{2}{|c|}{ Solid fraction } & \multirow{2}{*}{$\begin{array}{c}\text { Total } \mathrm{Na} \\
\text { Estimated* }\end{array}$} & \multirow{2}{*}{$\begin{array}{c}\text { Total } \mathrm{SO}_{4} \\
\text { Estimated* }\end{array}$} & \multirow{3}{*}{$\begin{array}{c}\mathrm{SO}_{4} / \mathrm{Na} \\
\text { Estimated* }\end{array}$} \\
\hline & $\mathrm{Na}$ & $\mathrm{SO}_{4}$ & $\mathrm{Na}$ & $\mathrm{SO}_{4}$ & $\mathrm{Na}$ & $\mathrm{SO}_{4}$ & $\mathrm{Na}$ & $\mathrm{SO}_{4}$ & & & \\
\hline Units & $\mathrm{Kg}$ & $\mathrm{kg}$ & $\mathrm{kg}$ & $\mathrm{kg}$ & mol. & mol. & mol. & mol. & mol. & mol. & \\
\hline BX-103 & $3.19 \mathrm{E}+03$ & $0.00 \mathrm{E}+00$ & $4.36 \mathrm{E}+04$ & $2.02 \mathrm{E}+03$ & $1.39 \mathrm{E}-01$ & $0.00 \mathrm{E}+00$ & $1.90 \mathrm{E}+00$ & $2.10 \mathrm{E}-02$ & $1.09 \mathrm{E}+00$ & $1.58 \mathrm{E}-02$ & $1.45 \mathrm{E}-02$ \\
\hline BX-104 & $4.77 \mathrm{E}+03$ & $1.34 \mathrm{E}+02$ & $4.57 \mathrm{E}+04$ & $1.10 \mathrm{E}+03$ & 2.07E-01 & $1.39 \mathrm{E}-03$ & $1.99 \mathrm{E}+00$ & $1.15 \mathrm{E}-02$ & $1.20 \mathrm{E}+00$ & $9.98 \mathrm{E}-03$ & $8.31 \mathrm{E}-03$ \\
\hline BX-105 & $2.35 \mathrm{E}+03$ & $7.39 \mathrm{E}+01$ & $3.24 \mathrm{E}+04$ & $5.15 \mathrm{E}+02$ & $1.02 \mathrm{E}-01$ & 7.69E-04 & $1.41 \mathrm{E}+00$ & $5.36 \mathrm{E}-03$ & $8.07 \mathrm{E}-01$ & 4.79E-03 & $5.94 \mathrm{E}-03$ \\
\hline BX-106 & $0.00 \mathrm{E}+00$ & $0.00 \mathrm{E}+00$ & $2.59 \mathrm{E}+04$ & $4.13 \mathrm{E}+02$ & $0.00 \mathrm{E}+00$ & $0.00 \mathrm{E}+00$ & $1.13 \mathrm{E}+00$ & $4.30 \mathrm{E}-03$ & $5.63 \mathrm{E}-01$ & $3.22 \mathrm{E}-03$ & $5.72 \mathrm{E}-03$ \\
\hline BX-107 & $0.00 \mathrm{E}+00$ & $0.00 \mathrm{E}+00$ & $1.91 \mathrm{E}+05$ & $2.51 \mathrm{E}+04$ & $0.00 \mathrm{E}+00$ & $0.00 \mathrm{E}+00$ & $8.31 \mathrm{E}+00$ & 2.61E-01 & $4.15 \mathrm{E}+00$ & $1.96 \mathrm{E}-01$ & $4.72 \mathrm{E}-02$ \\
\hline BX-108 & $0.00 \mathrm{E}+00$ & $0.00 \mathrm{E}+00$ & $1.50 \mathrm{E}+04$ & $2.33 \mathrm{E}+03$ & $0.00 \mathrm{E}+00$ & $0.00 \mathrm{E}+00$ & $52 \mathrm{E}-01$ & $2.43 \mathrm{E}-02$ & $3.26 \mathrm{E}-01$ & $1.82 \mathrm{E}-02$ & $5.58 \mathrm{E}-02$ \\
\hline BX-109 & $0.00 \mathrm{E}+00$ & $0.00 \mathrm{E}+00$ & $1.14 \mathrm{E}+05$ & $1.90 \mathrm{E}+04$ & $0.00 \mathrm{E}+00$ & $0.00 \mathrm{E}+00$ & $4.96 \mathrm{E}+00$ & $1.98 \mathrm{E}-01$ & $2.48 \mathrm{E}+00$ & $1.48 \mathrm{E}-01$ & $98 \mathrm{E}-02$ \\
\hline BX-110 & $2.43 \mathrm{E}+03$ & $2.59 \mathrm{E}+01$ & $2.62 \mathrm{E}+05$ & $9.82 \mathrm{E}+03$ & $1.06 \mathrm{E}-01$ & $2.70 \mathrm{E}-04$ & $1.14 \mathrm{E}+01$ & $1.02 \mathrm{E}-01$ & $5.80 \mathrm{E}+00$ & 7.69E-02 & $1.33 \mathrm{E}-02$ \\
\hline BX-111 & $0.00 \mathrm{E}+00$ & $0.00 \mathrm{E}+00$ & $2.15 \mathrm{E}+05$ & $4.10 \mathrm{E}+03$ & $0.00 \mathrm{E}+00$ & $0.00 \mathrm{E}+00$ & $9.35 \mathrm{E}+00$ & $4.27 \mathrm{E}-02$ & $4.68 \mathrm{E}+00$ & $3.20 \mathrm{E}-02$ & $6.85 \mathrm{E}-03$ \\
\hline BX-112 & $2.96 \mathrm{E}+02$ & $2.73 \mathrm{E}+01$ & $6.65 \mathrm{E}+04$ & $5.26 \mathrm{E}+03$ & $1.29 \mathrm{E}-02$ & $2.84 \mathrm{E}-04$ & $2.89 \mathrm{E}+00$ & $5.48 \mathrm{E}-02$ & $1.46 \mathrm{E}+00$ & $4.14 \mathrm{E}-02$ & $2.83 \mathrm{E}-02$ \\
\hline BY-101 & $0.00 \mathrm{E}+00$ & $0.00 \mathrm{E}+00$ & $4.64 \mathrm{E}+05$ & $5.43 \mathrm{E}+04$ & $0.00 \mathrm{E}+00$ & $0.00 \mathrm{E}+00$ & $2.02 \mathrm{E}+01$ & $5.65 \mathrm{E}-01$ & $1.01 \mathrm{E}+01$ & 4.24E-01 & $4.20 \mathrm{E}-02$ \\
\hline BY-102 & $1.01 \mathrm{E}+04$ & $4.58 \mathrm{E}+02$ & $4.20 \mathrm{E}+05$ & $9.08 \mathrm{E}+04$ & 4.39E-01 & $4.77 \mathrm{E}-03$ & $1.83 \mathrm{E}+01$ & $9.45 \mathrm{E}-01$ & $9.57 \mathrm{E}+00$ & $7.14 \mathrm{E}-01$ & 7.45E-02 \\
\hline BY-103 & $0.00 \mathrm{E}+00$ & $0.00 \mathrm{E}+00$ & $5.44 \mathrm{E}+05$ & $6.36 \mathrm{E}+04$ & $0.00 \mathrm{E}+00$ & $0.00 \mathrm{E}+00$ & $2.37 \mathrm{E}+01$ & $6.62 \mathrm{E}-01$ & $1.18 \mathrm{E}+01$ & 4.97E-01 & $4.20 \mathrm{E}-02$ \\
\hline BY-104 & $0.00 \mathrm{E}+00$ & $0.00 \mathrm{E}+00$ & $4.75 E+05$ & $3.74 \mathrm{E}+04$ & $0.00 \mathrm{E}+00$ & $0.00 \mathrm{E}+00$ & $2.07 \mathrm{E}+01$ & $3.89 \mathrm{E}-01$ & $1.03 \mathrm{E}+01$ & $2.92 \mathrm{E}-01$ & $2.83 \mathrm{E}-02$ \\
\hline BY-105 & $8.47 \mathrm{E}+04$ & $1.04 \mathrm{E}+03$ & $6.52 \mathrm{E}+05$ & $2.23 \mathrm{E}+04$ & $3.68 \mathrm{E}+00$ & $1.08 \mathrm{E}-02$ & $2.84 \mathrm{E}+01$ & 2.32E-01 & $1.79 \mathrm{E}+01$ & $1.85 \mathrm{E}-01$ & $1.04 \mathrm{E}-02$ \\
\hline BY-106 & $3.71 \mathrm{E}+05$ & $3.82 \mathrm{E}+03$ & $4.60 \mathrm{E}+05$ & $3.62 \mathrm{E}+04$ & $1.61 \mathrm{E}+01$ & $3.98 \mathrm{E}-02$ & $2.00 \mathrm{E}+01$ & $3.77 \mathrm{E}-01$ & $2.61 \mathrm{E}+01$ & $3.22 \mathrm{E}-01$ & $1.23 \mathrm{E}-02$ \\
\hline BY-107 & $3.37 \mathrm{E}+04$ & $2.66 \mathrm{E}+01$ & $4.50 \mathrm{E}+05$ & $3.14 \mathrm{E}+04$ & $1.47 \mathrm{E}+00$ & $2.77 \mathrm{E}-04$ & $1.96 \mathrm{E}+01$ & $3.27 \mathrm{E}-01$ & $1.13 \mathrm{E}+01$ & $2.45 \mathrm{E}-01$ & $2.18 \mathrm{E}-02$ \\
\hline BY-108 & $0.00 \mathrm{E}+00$ & $0.00 \mathrm{E}+00$ & $2.12 \mathrm{E}+05$ & $3.05 \mathrm{E}+04$ & $0.00 \mathrm{E}+00$ & $0.00 \mathrm{E}+00$ & $9.22 \mathrm{E}+00$ & $3.18 \mathrm{E}-01$ & $4.61 \mathrm{E}+00$ & $2.38 \mathrm{E}-01$ & $5.16 \mathrm{E}-02$ \\
\hline BY-109 & $5.72 \mathrm{E}+04$ & $6.16 \mathrm{E}+03$ & $3.06 \mathrm{E}+05$ & $1.08 \mathrm{E}+05$ & $2.49 \mathrm{E}+00$ & $6.41 \mathrm{E}-02$ & $1.33 \mathrm{E}+01$ & $1.12 \mathrm{E}+00$ & $9.14 \mathrm{E}+00$ & $9.07 \mathrm{E}-01$ & $9.92 \mathrm{E}-02$ \\
\hline BY-110 & $0.00 \mathrm{E}+00$ & $0.00 \mathrm{E}+00$ & $5.70 \mathrm{E}+05$ & $4.79 \mathrm{E}+04$ & $0.00 \mathrm{E}+00$ & $0.00 \mathrm{E}+00$ & $2.48 \mathrm{E}+01$ & 4.99E-01 & $1.24 \mathrm{E}+01$ & 3.74E-01 & $3.02 \mathrm{E}-02$ \\
\hline BY-111 & $0.00 \mathrm{E}+00$ & $0.00 \mathrm{E}+00$ & $6.57 \mathrm{E}+05$ & $9.38 \mathrm{E}+04$ & $0.00 \mathrm{E}+00$ & $0.00 \mathrm{E}+00$ & $2.86 \mathrm{E}+01$ & $9.76 \mathrm{E}-01$ & $1.43 \mathrm{E}+01$ & 7.32E-01 & $5.13 \mathrm{E}-02$ \\
\hline BY-112 & $0.00 \mathrm{E}+00$ & $0.00 \mathrm{E}+00$ & $5.37 \mathrm{E}+05$ & $4.02 \mathrm{E}+04$ & $0.00 \mathrm{E}+00$ & $0.00 \mathrm{E}+00$ & $2.34 \mathrm{E}+01$ & $4.18 \mathrm{E}-01$ & $1.17 \mathrm{E}+01$ & $3.14 \mathrm{E}-01$ & 2.69E-02 \\
\hline C-101 & $0.00 \mathrm{E}+00$ & $0.00 \mathrm{E}+00$ & $4.51 \mathrm{E}+04$ & $1.01 \mathrm{E}+03$ & $0.00 \mathrm{E}+00$ & $0.00 \mathrm{E}+00$ & $1.96 \mathrm{E}+00$ & $1.05 \mathrm{E}-02$ & $9.81 \mathrm{E}-01$ & $7.89 \mathrm{E}-03$ & $8.04 \mathrm{E}-03$ \\
\hline C-102 & $0.00 \mathrm{E}+00$ & $0.00 \mathrm{E}+00$ & $1.35 \mathrm{E}+05$ & $5.68 \mathrm{E}+03$ & $0.00 \mathrm{E}+00$ & $0.00 \mathrm{E}+00$ & $5.87 \mathrm{E}+00$ & $5.91 \mathrm{E}-02$ & $2.94 \mathrm{E}+00$ & $4.43 \mathrm{E}-02$ & $1.51 \mathrm{E}-02$ \\
\hline C-103 & $1.11 \mathrm{E}+04$ & $1.02 \mathrm{E}+03$ & $2.98 \mathrm{E}+04$ & $1.63 \mathrm{E}+03$ & $4.83 \mathrm{E}-01$ & $1.06 \mathrm{E}-02$ & $1.30 \mathrm{E}+00$ & $1.70 \mathrm{E}-02$ & $1.13 \mathrm{E}+00$ & $2.33 \mathrm{E}-02$ & $2.06 \mathrm{E}-02$ \\
\hline C-104 & $0.00 \mathrm{E}+00$ & $0.00 \mathrm{E}+00$ & $1.82 \mathrm{E}+05$ & $4.56 \mathrm{E}+03$ & $0.00 \mathrm{E}+00$ & $0.00 \mathrm{E}+00$ & $7.92 \mathrm{E}+00$ & 4.75E-02 & $3.96 \mathrm{E}+00$ & $3.56 \mathrm{E}-02$ & $8.99 \mathrm{E}-03$ \\
\hline C-105 & $8.74 \mathrm{E}+03$ & $1.44 \mathrm{E}+03$ & $4.67 \mathrm{E}+04$ & $1.33 \mathrm{E}+03$ & $3.80 \mathrm{E}-01$ & $1.50 \mathrm{E}-02$ & $2.03 \mathrm{E}+00$ & $1.38 \mathrm{E}-02$ & $1.40 \mathrm{E}+00$ & $2.54 \mathrm{E}-02$ & $1.82 \mathrm{E}-02$ \\
\hline C-106 & $1.02 \mathrm{E}+04$ & $4.37 \mathrm{E}+02$ & $2.81 \mathrm{E}+03$ & $7.74 \mathrm{E}+01$ & 4.44E-01 & $4.55 \mathrm{E}-03$ & $1.22 \mathrm{E}-01$ & $8.06 \mathrm{E}-04$ & $5.05 \mathrm{E}-01$ & $5.15 \mathrm{E}-03$ & $1.02 \mathrm{E}-02$ \\
\hline C-107 & $0.00 \mathrm{E}+00$ & $0.00 \mathrm{E}+00$ & $8.01 \mathrm{E}+04$ & $5.63 \mathrm{E}+03$ & $0.00 \mathrm{E}+00$ & $0.00 \mathrm{E}+00$ & $3.48 \mathrm{E}+00$ & $5.86 \mathrm{E}-02$ & $1.74 \mathrm{E}+00$ & $4.40 \mathrm{E}-02$ & $2.52 \mathrm{E}-02$ \\
\hline C-108 & $0.00 \mathrm{E}+00$ & $0.00 \mathrm{E}+00$ & $3.29 \mathrm{E}+04$ & $2.92 \mathrm{E}+03$ & $0.00 \mathrm{E}+00$ & $0.00 \mathrm{E}+00$ & $1.43 \mathrm{E}+00$ & $3.04 \mathrm{E}-02$ & 7.16E-01 & $2.28 \mathrm{E}-02$ & $3.19 \mathrm{E}-02$ \\
\hline
\end{tabular}

*generated with the assumption that $50 \%$ of sodium in the solid fraction and $75 \%$ of the sulfate would be returned to the liquid feed. 
Appendix A2: Estimate of the Sulfate to Sodium Ratio Expected in the LAW Feed Delivered for 149 Single Shell Tanks Per TFCOUP Rev. 3A (cont'd).

\begin{tabular}{|c|c|c|c|c|c|c|c|c|c|c|c|}
\hline Species & \multicolumn{2}{|c|}{ Liquid fraction } & \multicolumn{2}{|c|}{ Solid fraction } & \multicolumn{2}{|c|}{ Liquid fraction } & \multicolumn{2}{|c|}{ Solid fraction } & \multirow{2}{*}{$\begin{array}{c}\text { Total Na } \\
\text { Estimated* }\end{array}$} & \multirow{2}{*}{$\begin{array}{c}\text { Total } \mathrm{SO}_{4} \\
\text { Estimated* }\end{array}$} & \multirow{3}{*}{$\begin{array}{c}\mathrm{SO}_{4} / \mathrm{Na} \\
\text { Estimated* }\end{array}$} \\
\hline & $\mathrm{Na}$ & $\mathrm{SO}_{4}$ & $\mathrm{Na}$ & $\mathrm{SO}_{4}$ & $\mathrm{Na}$ & $\mathrm{SO}_{4}$ & $\mathrm{Na}$ & $\mathrm{SO}_{4}$ & & & \\
\hline Units & $\mathrm{Kg}$ & $\mathrm{kg}$ & $\mathrm{kg}$ & $\mathrm{kg}$ & mol. & mol. & mol. & $\mathrm{mol}$ & mol. & $\mathrm{mol}$ & \\
\hline C-109 & $0.00 \mathrm{E}+00$ & $0.00 \mathrm{E}+00$ & $2.54 \mathrm{E}+04$ & $2.23 \mathrm{E}+03$ & $0.00 \mathrm{E}+00$ & $0.00 \mathrm{E}+00$ & $1.10 \mathrm{E}+00$ & $2.32 \mathrm{E}-02$ & $5.52 \mathrm{E}-01$ & $1.74 \mathrm{E}-02$ & $3.15 \mathrm{E}-02$ \\
\hline C-110 & $0.00 \mathrm{E}+00$ & $0.00 \mathrm{E}+00$ & $8.08 \mathrm{E}+04$ & $1.45 \mathrm{E}+04$ & $0.00 \mathrm{E}+00$ & $0.00 \mathrm{E}+00$ & $3.51 \mathrm{E}+00$ & $1.51 \mathrm{E}-01$ & $1.76 \mathrm{E}+00$ & $1.13 \mathrm{E}-01$ & $6.44 \mathrm{E}-02$ \\
\hline $\mathrm{C}-111$ & $0.00 \mathrm{E}+00$ & $0.00 \mathrm{E}+00$ & $7.58 \mathrm{E}+03$ & $8.24 \mathrm{E}+02$ & $0.00 \mathrm{E}+00$ & $0.00 \mathrm{E}+00$ & $3.30 \mathrm{E}-01$ & $8.58 \mathrm{E}-03$ & $1.65 \mathrm{E}-01$ & $6.43 \mathrm{E}-03$ & $3.90 \mathrm{E}-02$ \\
\hline C-112 & $0.00 \mathrm{E}+00$ & $0.00 \mathrm{E}+00$ & $7.66 \mathrm{E}+04$ & $9.29 \mathrm{E}+03$ & $0.00 \mathrm{E}+00$ & $0.00 \mathrm{E}+00$ & $3.33 \mathrm{E}+00$ & $9.67 \mathrm{E}-02$ & $1.67 \mathrm{E}+00$ & $7.25 \mathrm{E}-02$ & $4.35 \mathrm{E}-02$ \\
\hline $\mathrm{C}-201$ & $0.00 \mathrm{E}+00$ & $0.00 \mathrm{E}+00$ & $8.79 \mathrm{E}+02$ & $2.17 \mathrm{E}+01$ & $0.00 \mathrm{E}+00$ & $0.00 \mathrm{E}+00$ & 3.82E-02 & $2.26 \mathrm{E}-04$ & $1.91 \mathrm{E}-02$ & $1.69 \mathrm{E}-04$ & $8.86 \mathrm{E}-03$ \\
\hline C-202 & $.00 \mathrm{E}+00$ & $0.00 \mathrm{E}+00$ & $4.59 \mathrm{E}+02$ & $2.87 \mathrm{E}+01$ & $0.00 \mathrm{E}+00$ & $0.00 \mathrm{E}+00$ & $2.00 \mathrm{E}-02$ & $2.99 \mathrm{E}-04$ & $9.98 \mathrm{E}-03$ & $2.24 \mathrm{E}-04$ & $2.24 \mathrm{E}-02$ \\
\hline $\mathrm{C}-203$ & $0.00 \mathrm{E}+00$ & $0.00 \mathrm{E}+00$ & $1.59 \mathrm{E}+03$ & $1.51 \mathrm{E}+02$ & $0.00 \mathrm{E}+00$ & $0.00 \mathrm{E}+00$ & $6.92 \mathrm{E}-02$ & $1.57 \mathrm{E}-03$ & $3.46 \mathrm{E}-02$ & $1.18 \mathrm{E}-03$ & $3.41 \mathrm{E}-02$ \\
\hline C-204 & $0.00 \mathrm{E}+00$ & $0.00 \mathrm{E}+00$ & $6.67 \mathrm{E}+02$ & $8.58 \mathrm{E}+01$ & $0.00 \mathrm{E}+00$ & $0.00 \mathrm{E}+00$ & $2.90 \mathrm{E}-02$ & 8.93E-04 & $1.45 \mathrm{E}-02$ & $6.70 \mathrm{E}-04$ & $4.62 \mathrm{E}-02$ \\
\hline S-101 & $6.26 \mathrm{E}+04$ & $1.65 \mathrm{E}+03$ & $3.33 \mathrm{E}+05$ & $1.74 \mathrm{E}+04$ & $2.72 \mathrm{E}+00$ & $1.72 \mathrm{E}-02$ & $1.45 \mathrm{E}+01$ & $1.81 \mathrm{E}-01$ & $9.97 \mathrm{E}+00$ & $1.53 \mathrm{E}-01$ & $1.54 \mathrm{E}-02$ \\
\hline S-102 & $5.04 \mathrm{E}+04$ & $9.90 \mathrm{E}+02$ & $3.60 \mathrm{E}+05$ & $1.71 \mathrm{E}+04$ & $2.19 \mathrm{E}+00$ & $1.03 \mathrm{E}-02$ & $1.57 \mathrm{E}+01$ & $1.78 \mathrm{E}-01$ & $1.00 \mathrm{E}+01$ & 1.44E-01 & $1.44 \mathrm{E}-02$ \\
\hline S-103 & $3.97 \mathrm{E}+04$ & $8.87 \mathrm{E}+02$ & $2.29 \mathrm{E}+05$ & $1.41 \mathrm{E}+04$ & $1.73 \mathrm{E}+00$ & $9.23 \mathrm{E}-03$ & $9.96 \mathrm{E}+00$ & $1.47 \mathrm{E}-01$ & $6.71 \mathrm{E}+00$ & $1.19 \mathrm{E}-01$ & $1.78 \mathrm{E}-02$ \\
\hline S-104 & $0.00 \mathrm{E}+00$ & $0.00 \mathrm{E}+00$ & $2.21 \mathrm{E}+05$ & $4.14 \mathrm{E}+03$ & $0.00 \mathrm{E}+00$ & $0.00 \mathrm{E}+00$ & $9.61 \mathrm{E}+00$ & $4.31 \mathrm{E}-02$ & $4.81 \mathrm{E}+00$ & $3.23 \mathrm{E}-02$ & $6.73 \mathrm{E}-03$ \\
\hline S-105 & $0.00 \mathrm{E}+00$ & $0.00 \mathrm{E}+00$ & $5.80 \mathrm{E}+05$ & $3.89 \mathrm{E}+04$ & $0.00 \mathrm{E}+00$ & $0.00 \mathrm{E}+00$ & $2.52 \mathrm{E}+01$ & $4.05 \mathrm{E}-01$ & $1.26 \mathrm{E}+01$ & 3.04E-01 & $2.41 \mathrm{E}-02$ \\
\hline S-106 & $1.39 \mathrm{E}+04$ & $3.27 \mathrm{E}+02$ & $4.85 \mathrm{E}+05$ & $1.84 \mathrm{E}+04$ & $6.05 \mathrm{E}-01$ & $3.40 \mathrm{E}-03$ & $2.11 \mathrm{E}+01$ & $1.92 \mathrm{E}-01$ & $1.12 \mathrm{E}+01$ & $1.47 \mathrm{E}-01$ & $1.32 \mathrm{E}-02$ \\
\hline S-107 & $1.37 \mathrm{E}+05$ & $5.93 \mathrm{E}+03$ & $7.49 \mathrm{E}+04$ & $0.00 \mathrm{E}+00$ & $5.96 \mathrm{E}+00$ & $6.17 \mathrm{E}-02$ & $3.26 \mathrm{E}+00$ & $0.00 \mathrm{E}+00$ & $7.59 \mathrm{E}+00$ & $6.17 \mathrm{E}-02$ & $8.14 \mathrm{E}-03$ \\
\hline S-108 & $0.00 \mathrm{E}+00$ & $0.00 \mathrm{E}+00$ & $5.47 \mathrm{E}+05$ & $3.66 \mathrm{E}+04$ & $0.00 \mathrm{E}+00$ & $0.00 \mathrm{E}+00$ & $2.38 \mathrm{E}+01$ & $3.81 \mathrm{E}-01$ & $1.19 \mathrm{E}+01$ & $2.86 \mathrm{E}-01$ & $2.40 \mathrm{E}-02$ \\
\hline S-109 & $1.05 \mathrm{E}+05$ & $8.19 \mathrm{E}+02$ & $5.95 \mathrm{E}+05$ & $1.88 \mathrm{E}+04$ & $4.57 \mathrm{E}+00$ & $8.53 \mathrm{E}-03$ & $2.59 \mathrm{E}+01$ & $1.96 \mathrm{E}-01$ & $1.75 \mathrm{E}+01$ & $1.55 \mathrm{E}-01$ & $8.87 \mathrm{E}-03$ \\
\hline S-110 & $2.41 \mathrm{E}+04$ & $6.43 \mathrm{E}+02$ & $4.46 \mathrm{E}+05$ & $1.99 \mathrm{E}+04$ & $1.05 \mathrm{E}+00$ & $6.69 \mathrm{E}-03$ & $1.94 \mathrm{E}+01$ & $2.07 \mathrm{E}-01$ & $1.07 \mathrm{E}+01$ & 1.62E-01 & $1.51 \mathrm{E}-02$ \\
\hline S-111 & $1.08 \mathrm{E}+05$ & $2.13 \mathrm{E}+03$ & $3.37 \mathrm{E}+05$ & $2.81 \mathrm{E}+04$ & $4.70 \mathrm{E}+00$ & $2.22 \mathrm{E}-02$ & $1.47 \mathrm{E}+01$ & $2.93 \mathrm{E}-01$ & $1.20 \mathrm{E}+01$ & 2.42E-01 & $2.01 \mathrm{E}-02$ \\
\hline S-112 & $5.08 \mathrm{E}+05$ & $4.22 \mathrm{E}+04$ & $1.55 \mathrm{E}+05$ & $2.16 \mathrm{E}+03$ & $2.21 \mathrm{E}+01$ & $4.39 \mathrm{E}-01$ & $6.74 \mathrm{E}+00$ & $2.25 \mathrm{E}-02$ & $2.55 \mathrm{E}+01$ & 4.56E-01 & $1.79 \mathrm{E}-02$ \\
\hline SX-101 & $1.51 \mathrm{E}+05$ & $1.14 \mathrm{E}+03$ & $4.04 \mathrm{E}+05$ & $4.58 \mathrm{E}+03$ & $6.57 \mathrm{E}+00$ & 1.19E-02 & $1.76 \mathrm{E}+01$ & 4.77E-02 & $1.54 \mathrm{E}+01$ & 4.76E-02 & $3.10 \mathrm{E}-03$ \\
\hline SX-102 & $1.20 \mathrm{E}+05$ & $1.70 \mathrm{E}+03$ & $5.09 \mathrm{E}+05$ & $3.30 \mathrm{E}+04$ & $5.22 \mathrm{E}+00$ & $1.77 \mathrm{E}-02$ & $2.21 \mathrm{E}+01$ & $3.44 \mathrm{E}-01$ & $1.63 \mathrm{E}+01$ & 2.75E-01 & $1.69 \mathrm{E}-02$ \\
\hline SX-103 & $2.33 \mathrm{E}+05$ & $1.98 \mathrm{E}+03$ & $4.67 \mathrm{E}+05$ & $3.29 \mathrm{E}+04$ & $1.01 \mathrm{E}+01$ & $2.06 \mathrm{E}-02$ & $2.03 \mathrm{E}+01$ & $3.42 \mathrm{E}-01$ & $2.03 \mathrm{E}+01$ & $2.77 \mathrm{E}-01$ & $1.37 \mathrm{E}-02$ \\
\hline SX-104 & $4.45 \mathrm{E}+04$ & $6.93 \mathrm{E}+02$ & $4.38 \mathrm{E}+05$ & $2.29 \mathrm{E}+04$ & $1.94 \mathrm{E}+00$ & $7.21 \mathrm{E}-03$ & $1.91 \mathrm{E}+01$ & $2.38 \mathrm{E}-01$ & $1.15 \mathrm{E}+01$ & $1.86 \mathrm{E}-01$ & $1.62 \mathrm{E}-02$ \\
\hline SX-105 & $2.56 \mathrm{E}+05$ & $4.55 \mathrm{E}+03$ & $4.08 \mathrm{E}+05$ & $2.12 \mathrm{E}+04$ & $1.11 \mathrm{E}+01$ & 4.74E-02 & $1.77 \mathrm{E}+01$ & $2.21 \mathrm{E}-01$ & $2.00 \mathrm{E}+01$ & 2.13E-01 & $1.06 \mathrm{E}-02$ \\
\hline SX-106 & $3.45 \mathrm{E}+04$ & $1.02 \mathrm{E}+03$ & $4.14 \mathrm{E}+05$ & $9.98 \mathrm{E}+03$ & $1.50 \mathrm{E}+00$ & $1.06 \mathrm{E}-02$ & $1.80 \mathrm{E}+01$ & $1.04 \mathrm{E}-01$ & $1.05 \mathrm{E}+01$ & $8.85 \mathrm{E}-02$ & $8.43 \mathrm{E}-03$ \\
\hline SX-107 & $0.00 \mathrm{E}+00$ & $0.00 \mathrm{E}+00$ & $7.96 \mathrm{E}+04$ & $1.37 \mathrm{E}+03$ & $0.00 \mathrm{E}+00$ & $0.00 \mathrm{E}+00$ & $3.46 \mathrm{E}+00$ & $1.43 \mathrm{E}-02$ & $1.73 \mathrm{E}+00$ & $1.07 \mathrm{E}-02$ & $6.18 \mathrm{E}-03$ \\
\hline SX-108 & $0.00 \mathrm{E}+00$ & $0.00 \mathrm{E}+00$ & $5.53 \mathrm{E}+04$ & $3.97 \mathrm{E}+03$ & $0.00 \mathrm{E}+00$ & $0.00 \mathrm{E}+00$ & $2.41 \mathrm{E}+00$ & 4.13E-02 & $1.20 \mathrm{E}+00$ & $3.10 \mathrm{E}-02$ & $2.58 \mathrm{E}-02$ \\
\hline SX-109 & $0.00 \mathrm{E}+00$ & $0.00 \mathrm{E}+00$ & $2.66 \mathrm{E}+05$ & $2.46 \mathrm{E}+03$ & $0.00 \mathrm{E}+00$ & $0.00 \mathrm{E}+00$ & $1.16 \mathrm{E}+01$ & $2.56 \mathrm{E}-02$ & $5.79 \mathrm{E}+00$ & $1.92 \mathrm{E}-02$ & $3.32 \mathrm{E}-03$ \\
\hline SX-110 & $0.00 \mathrm{E}+00$ & $0.00 \mathrm{E}+00$ & $4.35 \mathrm{E}+04$ & $8.91 \mathrm{E}+02$ & $0.00 \mathrm{E}+00$ & $0.00 \mathrm{E}+00$ & $1.89 \mathrm{E}+00$ & $9.28 \mathrm{E}-03$ & $9.46 \mathrm{E}-01$ & $6.96 \mathrm{E}-03$ & $7.35 \mathrm{E}-03$ \\
\hline
\end{tabular}

*generated with the assumption that $50 \%$ of sodium in the solid fraction and $75 \%$ of the sulfate would be returned to the liquid feed. 
Appendix A2: Estimate of the Sulfate to Sodium Ratio Expected in the LAW Feed Delivered for 149 Single Shell Tanks Per TFCOUP Rev. 3A (cont'd).

\begin{tabular}{|c|c|c|c|c|c|c|c|c|c|c|c|}
\hline \multirow[b]{2}{*}{ Species } & \multicolumn{2}{|c|}{ Liquid fraction } & \multicolumn{2}{|c|}{ Solid fraction } & \multicolumn{2}{|c|}{ Liquid fraction } & \multicolumn{2}{|c|}{ Solid fraction } & \multirow{2}{*}{$\begin{array}{c}\text { Total Na } \\
\text { Estimated* }\end{array}$} & \multirow{2}{*}{$\begin{array}{c}\text { Total } \mathrm{SO}_{4} \\
\text { Estimated* }\end{array}$} & \multirow{3}{*}{$\begin{array}{c}\mathrm{SO}_{4} / \mathrm{Na} \\
\text { Estimated* }\end{array}$} \\
\hline & $\mathrm{Na}$ & $\mathrm{SO}_{4}$ & $\mathrm{Na}$ & $\mathrm{SO}_{4}$ & $\mathrm{Na}$ & $\mathrm{SO}_{4}$ & $\mathrm{Na}$ & & & & \\
\hline Units & $\mathrm{Kg}$ & $\mathrm{kg}$ & $\mathrm{kg}$ & $\mathrm{kg}$ & mol. & mol. & mol. & mol. & mol. & mol. & \\
\hline SX-111 & $0.00 \mathrm{E}+00$ & $0.00 \mathrm{E}+00$ & $8.57 \mathrm{E}+04$ & $1.75 \mathrm{E}+03$ & $0.00 \mathrm{E}+00$ & $0.00 \mathrm{E}+00$ & $3.73 \mathrm{E}+00$ & $1.82 \mathrm{E}-02$ & $1.86 \mathrm{E}+00$ & $1.37 \mathrm{E}-02$ & $7.33 \mathrm{E}-03$ \\
\hline SX-112 & $0.00 \mathrm{E}+00$ & $0.00 \mathrm{E}+00$ & $7.56 \mathrm{E}+04$ & $1.55 \mathrm{E}+03$ & $0.00 \mathrm{E}+00$ & $0.00 \mathrm{E}+00$ & $3.29 \mathrm{E}+00$ & $1.61 \mathrm{E}-02$ & $1.64 \mathrm{E}+00$ & $1.21 \mathrm{E}-02$ & $7.36 \mathrm{E}-03$ \\
\hline SX-113 & $0.00 \mathrm{E}+00$ & $0.00 \mathrm{E}+00$ & $1.41 \mathrm{E}+03$ & $2.89 \mathrm{E}+01$ & $0.00 \mathrm{E}+00$ & $0.00 \mathrm{E}+00$ & $6.13 \mathrm{E}-02$ & $3.01 \mathrm{E}-04$ & $3.07 \mathrm{E}-02$ & $2.26 \mathrm{E}-04$ & $7.36 \mathrm{E}-03$ \\
\hline SX-114 & $0.00 \mathrm{E}+00$ & $0.00 \mathrm{E}+00$ & $1.74 \mathrm{E}+05$ & $1.65 \mathrm{E}+03$ & $0.00 \mathrm{E}+00$ & $0.00 \mathrm{E}+00$ & $7.57 \mathrm{E}+00$ & $1.72 \mathrm{E}-02$ & $3.78 \mathrm{E}+00$ & $1.29 \mathrm{E}-02$ & $3.40 \mathrm{E}-03$ \\
\hline SX-115 & $0.00 \mathrm{E}+00$ & $0.00 \mathrm{E}+00$ & $1.20 \mathrm{E}+04$ & $3.57 \mathrm{E}+02$ & $0.00 \mathrm{E}+00$ & $0.00 \mathrm{E}+00$ & $5.22 \mathrm{E}-01$ & $3.72 \mathrm{E}-03$ & $2.61 \mathrm{E}-01$ & $2.79 \mathrm{E}-03$ & $1.07 \mathrm{E}-02$ \\
\hline T-101 & $0.00 \mathrm{E}+00$ & $0.00 \mathrm{E}+00$ & $7.03 \mathrm{E}+04$ & $7.26 \mathrm{E}+02$ & $0.00 \mathrm{E}+00$ & $0.00 \mathrm{E}+00$ & $3.06 \mathrm{E}+00$ & $7.56 \mathrm{E}-03$ & $1.53 \mathrm{E}+00$ & $5.67 \mathrm{E}-03$ & $3.71 \mathrm{E}-03$ \\
\hline T-102 & $3.17 \mathrm{E}+03$ & $2.41 \mathrm{E}+02$ & $4.04 \mathrm{E}+03$ & $2.02 \mathrm{E}+02$ & $1.38 \mathrm{E}-01$ & $2.51 \mathrm{E}-03$ & $1.76 \mathrm{E}-01$ & $2.10 \mathrm{E}-03$ & $2.26 \mathrm{E}-01$ & $4.09 \mathrm{E}-03$ & $1.81 \mathrm{E}-02$ \\
\hline T-103 & $2.10 \mathrm{E}+03$ & $1.70 \mathrm{E}+02$ & $4.99 \mathrm{E}+03$ & $2.73 \mathrm{E}+02$ & $9.13 \mathrm{E}-02$ & $1.77 \mathrm{E}-03$ & $2.17 \mathrm{E}-01$ & $2.84 \mathrm{E}-03$ & $2.00 \mathrm{E}-01$ & $3.90 \mathrm{E}-03$ & $1.95 \mathrm{E}-02$ \\
\hline T-104 & $8.59 \mathrm{E}+03$ & $6.60 \mathrm{E}+02$ & $8.69 \mathrm{E}+04$ & $5.26 \mathrm{E}+03$ & $3.74 \mathrm{E}-01$ & $6.87 \mathrm{E}-03$ & $3.78 \mathrm{E}+00$ & $5.48 \mathrm{E}-02$ & $2.26 \mathrm{E}+00$ & 4.79E-02 & $2.12 \mathrm{E}-02$ \\
\hline T-105 & $8.21 \mathrm{E}+03$ & $1.37 \mathrm{E}+03$ & $2.63 \mathrm{E}+04$ & $3.89 \mathrm{E}+03$ & $3.57 \mathrm{E}-01$ & $1.43 \mathrm{E}-02$ & $1.14 \mathrm{E}+00$ & $4.05 \mathrm{E}-02$ & $9.29 \mathrm{E}-01$ & $4.46 \mathrm{E}-02$ & $4.80 \mathrm{E}-02$ \\
\hline T-106 & $0.00 \mathrm{E}+00$ & $0.00 \mathrm{E}+00$ & $9.18 \mathrm{E}+03$ & $9.08 \mathrm{E}+02$ & $0.00 \mathrm{E}+00$ & $0.00 \mathrm{E}+00$ & 3.99E-01 & $9.45 \mathrm{E}-03$ & $2.00 \mathrm{E}-01$ & $7.09 \mathrm{E}-03$ & $3.55 \mathrm{E}-02$ \\
\hline T-107 & $0.00 \mathrm{E}+00$ & $0.00 \mathrm{E}+00$ & $1.29 \mathrm{E}+05$ & $9.86 \mathrm{E}+03$ & $0.00 \mathrm{E}+00$ & $0.00 \mathrm{E}+00$ & $5.61 \mathrm{E}+00$ & $1.03 \mathrm{E}-01$ & $2.81 \mathrm{E}+00$ & $7.70 \mathrm{E}-02$ & $2.74 \mathrm{E}-02$ \\
\hline T-108 & $0.00 \mathrm{E}+00$ & $0.00 \mathrm{E}+00$ & $4.44 \mathrm{E}+04$ & $2.57 \mathrm{E}+03$ & $0.00 \mathrm{E}+00$ & $0.00 \mathrm{E}+00$ & $1.93 \mathrm{E}+00$ & $2.68 \mathrm{E}-02$ & $9.66 \mathrm{E}-01$ & $2.01 \mathrm{E}-02$ & $2.08 \mathrm{E}-02$ \\
\hline T-109 & $0.00 \mathrm{E}+00$ & $0.00 \mathrm{E}+00$ & $7.62 \mathrm{E}+04$ & $1.97 \mathrm{E}+02$ & $0.00 \mathrm{E}+00$ & $0.00 \mathrm{E}+00$ & $3.31 \mathrm{E}+00$ & $2.05 \mathrm{E}-03$ & $1.66 \mathrm{E}+00$ & $1.54 \mathrm{E}-03$ & $9.28 \mathrm{E}-04$ \\
\hline T-110 & $7.70 \mathrm{E}+03$ & $9.92 \mathrm{E}+02$ & $7.52 \mathrm{E}+04$ & $1.02 \mathrm{E}+04$ & $3.35 \mathrm{E}-01$ & 1.03E-02 & $3.27 \mathrm{E}+00$ & $1.06 \mathrm{E}-01$ & $1.97 \mathrm{E}+00$ & $9.00 \mathrm{E}-02$ & $4.57 \mathrm{E}-02$ \\
\hline $\mathrm{T}-111$ & $0.00 \mathrm{E}+00$ & $0.00 \mathrm{E}+00$ & $7.99 \mathrm{E}+04$ & $7.97 \mathrm{E}+03$ & $0.00 \mathrm{E}+00$ & $0.00 \mathrm{E}+00$ & $3.48 \mathrm{E}+00$ & $8.30 \mathrm{E}-02$ & $1.74 \mathrm{E}+00$ & $6.22 \mathrm{E}-02$ & $3.58 \mathrm{E}-02$ \\
\hline T-112 & $1.52 \mathrm{E}+03$ & $2.52 \mathrm{E}+02$ & $1.96 \mathrm{E}+04$ & $2.63 \mathrm{E}+03$ & $6.61 \mathrm{E}-02$ & $2.62 \mathrm{E}-03$ & $8.53 \mathrm{E}-01$ & $2.74 \mathrm{E}-02$ & 4.92E-01 & $2.32 \mathrm{E}-02$ & $4.70 \mathrm{E}-02$ \\
\hline T-201 & $0.00 \mathrm{E}+00$ & $0.00 \mathrm{E}+00$ & $4.50 \mathrm{E}+03$ & $3.86 \mathrm{E}+01$ & $0.00 \mathrm{E}+00$ & $0.00 \mathrm{E}+00$ & $1.96 \mathrm{E}-01$ & 4.02E-04 & 9.79E-02 & $3.01 \mathrm{E}-04$ & $3.08 \mathrm{E}-03$ \\
\hline T-202 & $0.00 \mathrm{E}+00$ & $0.00 \mathrm{E}+00$ & $3.54 \mathrm{E}+03$ & $1.09 \mathrm{E}+02$ & $0.00 \mathrm{E}+00$ & $0.00 \mathrm{E}+00$ & $1.54 \mathrm{E}-01$ & $1.13 \mathrm{E}-03$ & 7.70E-02 & $8.51 \mathrm{E}-04$ & $1.11 \mathrm{E}-02$ \\
\hline $\mathrm{T}-203$ & $0.00 \mathrm{E}+00$ & $0.00 \mathrm{E}+00$ & $5.67 \mathrm{E}+03$ & $7.14 \mathrm{E}+01$ & $0.00 \mathrm{E}+00$ & $0.00 \mathrm{E}+00$ & $2.47 \mathrm{E}-01$ & 7.43E-04 & $1.23 \mathrm{E}-01$ & $5.57 \mathrm{E}-04$ & $4.52 \mathrm{E}-03$ \\
\hline T-204 & $0.00 \mathrm{E}+00$ & $0.00 \mathrm{E}+00$ & $5.53 \mathrm{E}+03$ & $6.32 \mathrm{E}+01$ & $0.00 \mathrm{E}+00$ & $0.00 \mathrm{E}+00$ & $2.41 \mathrm{E}-01$ & $6.58 \mathrm{E}-04$ & $1.20 \mathrm{E}-01$ & 4.93E-04 & $4.10 \mathrm{E}-03$ \\
\hline TX-101 & $0.00 \mathrm{E}+00$ & $0.00 \mathrm{E}+00$ & $6.90 \mathrm{E}+04$ & $3.13 \mathrm{E}+03$ & $0.00 \mathrm{E}+00$ & $0.00 \mathrm{E}+00$ & $3.00 \mathrm{E}+00$ & $3.26 \mathrm{E}-02$ & $1.50 \mathrm{E}+00$ & $2.44 \mathrm{E}-02$ & $1.63 \mathrm{E}-02$ \\
\hline TX-102 & $0.00 \mathrm{E}+00$ & $0.00 \mathrm{E}+00$ & $2.85 \mathrm{E}+05$ & $3.44 \mathrm{E}+04$ & $0.00 \mathrm{E}+00$ & $0.00 \mathrm{E}+00$ & $1.24 \mathrm{E}+01$ & $3.58 \mathrm{E}-01$ & $6.20 \mathrm{E}+00$ & 2.69E-01 & $4.33 \mathrm{E}-02$ \\
\hline TX-103 & $0.00 \mathrm{E}+00$ & $0.00 \mathrm{E}+00$ & $2.06 \mathrm{E}+05$ & $2.47 \mathrm{E}+04$ & $0.00 \mathrm{E}+00$ & $0.00 \mathrm{E}+00$ & $8.96 \mathrm{E}+00$ & $2.57 \mathrm{E}-01$ & $4.48 \mathrm{E}+00$ & $1.93 \mathrm{E}-01$ & $4.30 \mathrm{E}-02$ \\
\hline TX-104 & $1.62 \mathrm{E}+04$ & $3.39 \mathrm{E}+02$ & $3.77 \mathrm{E}+04$ & $3.76 \mathrm{E}+02$ & 7.05E-01 & $3.53 \mathrm{E}-03$ & $1.64 \mathrm{E}+00$ & $3.91 \mathrm{E}-03$ & $1.52 \mathrm{E}+00$ & $6.46 \mathrm{E}-03$ & $4.24 \mathrm{E}-03$ \\
\hline TX-105 & $0.00 \mathrm{E}+00$ & $0.00 \mathrm{E}+00$ & $8.01 \mathrm{E}+05$ & $9.66 \mathrm{E}+04$ & $0.00 \mathrm{E}+00$ & $0.00 \mathrm{E}+00$ & $3.48 \mathrm{E}+01$ & $1.01 \mathrm{E}+00$ & $1.74 \mathrm{E}+01$ & 7.54E-01 & $4.33 \mathrm{E}-02$ \\
\hline TX-106 & $0.00 \mathrm{E}+00$ & $0.00 \mathrm{E}+00$ & $4.48 \mathrm{E}+05$ & $5.41 \mathrm{E}+04$ & $0.00 \mathrm{E}+00$ & $0.00 \mathrm{E}+00$ & $1.95 \mathrm{E}+01$ & $5.63 \mathrm{E}-01$ & $9.74 \mathrm{E}+00$ & 4.22E-01 & $4.34 \mathrm{E}-02$ \\
\hline TX-107 & $0.00 \mathrm{E}+00$ & $0.00 \mathrm{E}+00$ & $4.24 \mathrm{E}+04$ & $4.56 \mathrm{E}+03$ & $0.00 \mathrm{E}+00$ & $0.00 \mathrm{E}+00$ & $1.84 \mathrm{E}+00$ & $4.75 \mathrm{E}-02$ & $9.22 \mathrm{E}-01$ & $3.56 \mathrm{E}-02$ & $3.86 \mathrm{E}-02$ \\
\hline TX-108 & $0.00 \mathrm{E}+00$ & $0.00 \mathrm{E}+00$ & $1.72 \mathrm{E}+05$ & $2.09 \mathrm{E}+04$ & $0.00 \mathrm{E}+00$ & $0.00 \mathrm{E}+00$ & $7.48 \mathrm{E}+00$ & $2.18 \mathrm{E}-01$ & $3.74 \mathrm{E}+00$ & $1.63 \mathrm{E}-01$ & $4.36 \mathrm{E}-02$ \\
\hline TX-109 & $0.00 \mathrm{E}+00$ & $0.00 \mathrm{E}+00$ & $1.93 \mathrm{E}+05$ & $2.64 \mathrm{E}+04$ & $0.00 \mathrm{E}+00$ & $0.00 \mathrm{E}+00$ & $8.39 \mathrm{E}+00$ & $2.75 \mathrm{E}-01$ & $4.20 \mathrm{E}+00$ & $2.06 \mathrm{E}-01$ & $4.91 \mathrm{E}-02$ \\
\hline
\end{tabular}

"generated with the assumption that $50 \%$ of sodium in the solid fraction and $75 \%$ of the sulfate would be returned to the liquid feed. 
Appendix A2: Estimate of the Sulfate to Sodium Ratio Expected in the LAW Feed Delivered for 149 Single Shell Tanks Per TFCOUP Rev. 3A (cont'd).

\begin{tabular}{|c|c|c|c|c|c|c|c|c|c|c|c|}
\hline \multirow{2}{*}{ Species } & \multicolumn{2}{|c|}{ Liquid fraction } & \multicolumn{2}{|c|}{ Solid fraction } & \multicolumn{2}{|c|}{ Liquid fraction } & \multicolumn{2}{|c|}{ Solid fraction } & \multirow{2}{*}{$\begin{array}{c}\text { Total } \mathrm{Na} \\
\text { Estimated* }\end{array}$} & \multirow{2}{*}{$\begin{array}{l}\text { Total } \mathrm{SO}_{4} \\
\text { Estimated* }\end{array}$} & \multirow{3}{*}{$\begin{array}{c}\mathrm{SO}_{4} / \mathrm{Na} \\
\text { Estimated* }\end{array}$} \\
\hline & $\mathrm{Na}$ & $\mathrm{SO}_{4}$ & $\mathrm{Na}$ & $\mathrm{SO}_{4}$ & $\mathrm{Na}$ & $\mathrm{SO}_{4}$ & $\mathrm{Na}$ & & & & \\
\hline Units & $\mathrm{Kg}$ & $\mathrm{kg}$ & $\mathrm{kg}$ & $\mathrm{kg}$ & mol. & mol. & mol. & mol. & mol. & mol. & \\
\hline TX-110 & $0.00 \mathrm{E}+00$ & $0.00 \mathrm{E}+00$ & $5.78 \mathrm{E}+05$ & $7.00 \mathrm{E}+04$ & $0.00 \mathrm{E}+00$ & $0.00 \mathrm{E}+00$ & $2.51 \mathrm{E}+01$ & 7.29E-01 & $1.26 \mathrm{E}+01$ & $5.47 \mathrm{E}-01$ & $4.35 \mathrm{E}-02$ \\
\hline TX-111 & $0.00 \mathrm{E}+00$ & $0.00 \mathrm{E}+00$ & $4.52 \mathrm{E}+05$ & $5.49 \mathrm{E}+04$ & $0.00 \mathrm{E}+00$ & $0.00 \mathrm{E}+00$ & $1.97 \mathrm{E}+01$ & $5.72 \mathrm{E}-01$ & $83 \mathrm{E}+00$ & 4.29E-01 & $4.36 \mathrm{E}-02$ \\
\hline TX-112 & $0.00 \mathrm{E}+00$ & $0.00 \mathrm{E}+00$ & $8.52 \mathrm{E}+05$ & $1.01 \mathrm{E}+05$ & $0.00 \mathrm{E}+00$ & $0.00 \mathrm{E}+00$ & $3.71 \mathrm{E}+01$ & $1.05 \mathrm{E}+00$ & $1.85 \mathrm{E}+01$ & $7.89 \mathrm{E}-01$ & $4.26 \mathrm{E}-02$ \\
\hline TX-113 & $2.79 \mathrm{E}+04$ & $3.29 \mathrm{E}+03$ & $1.09 \mathrm{E}+06$ & $4.26 \mathrm{E}+05$ & $1.21 \mathrm{E}+00$ & $3.42 \mathrm{E}-02$ & $4.74 \mathrm{E}+01$ & $4.43 \mathrm{E}+00$ & $2.49 \mathrm{E}+01$ & $3.36 \mathrm{E}+00$ & $1.35 \mathrm{E}-01$ \\
\hline TX-114 & $0.00 \mathrm{E}+00$ & $0.00 \mathrm{E}+00$ & $6.98 \mathrm{E}+05$ & $8.01 \mathrm{E}+04$ & $0.00 \mathrm{E}+00$ & $0.00 \mathrm{E}+00$ & $3.04 \mathrm{E}+01$ & $8.34 \mathrm{E}-01$ & $1.52 \mathrm{E}+01$ & $6.25 \mathrm{E}-01$ & $4.12 \mathrm{E}-02$ \\
\hline TX-115 & $0.00 \mathrm{E}+00$ & $0.00 \mathrm{E}+00$ & $7.47 \mathrm{E}+05$ & $9.01 \mathrm{E}+04$ & $0.00 \mathrm{E}+00$ & $0.00 \mathrm{E}+00$ & $3.25 \mathrm{E}+01$ & $9.38 \mathrm{E}-01$ & $62 \mathrm{E}+01$ & $03 \mathrm{E}-01$ & $4.33 \mathrm{E}-02$ \\
\hline TX-116 & $0.00 \mathrm{E}+00$ & $0.00 \mathrm{E}+00$ & $7.96 \mathrm{E}+05$ & $9.95 \mathrm{E}+04$ & $0.00 \mathrm{E}+00$ & $0.00 \mathrm{E}+00$ & $3.46 \mathrm{E}+01$ & $1.04 \mathrm{E}+00$ & $1.73 \mathrm{E}+01$ & 7.77E-01 & 4.49E-02 \\
\hline TX-117 & $0.00 \mathrm{E}+00$ & $0.00 \mathrm{E}+00$ & $7.77 \mathrm{E}+05$ & $7.98 \mathrm{E}+04$ & $0.00 \mathrm{E}+00$ & $0.00 \mathrm{E}+00$ & $3.38 \mathrm{E}+01$ & $8.31 \mathrm{E}-01$ & $1.69 \mathrm{E}+01$ & $6.23 \mathrm{E}-01$ & $3.69 \mathrm{E}-02$ \\
\hline TX-118 & $0.00 \mathrm{E}+00$ & $0.00 \mathrm{E}+00$ & $3.78 \mathrm{E}+05$ & $1.19 \mathrm{E}+04$ & $0.00 \mathrm{E}+00$ & $0.00 \mathrm{E}+00$ & $1.64 \mathrm{E}+01$ & $1.24 \mathrm{E}-01$ & $8.22 \mathrm{E}+00$ & $9.29 \mathrm{E}-02$ & $1.13 \mathrm{E}-02$ \\
\hline TY-101 & $0.00 \mathrm{E}+00$ & $0.00 \mathrm{E}+00$ & $8.89 \mathrm{E}+04$ & $4.02 \mathrm{E}+03$ & $0.00 \mathrm{E}+00$ & $0.00 \mathrm{E}+00$ & $3.87 \mathrm{E}+00$ & $4.18 \mathrm{E}-02$ & & & $1.62 \mathrm{E}-02$ \\
\hline TY-102 & $0.00 \mathrm{E}+00$ & $0.00 \mathrm{E}+00$ & $9.38 \mathrm{E}+04$ & $7.94 \mathrm{E}+03$ & $0.00 \mathrm{E}+00$ & $0.00 \mathrm{E}+00$ & $4.08 \mathrm{E}+00$ & $8.27 \mathrm{E}-02$ & $2.04 \mathrm{E}+00$ & $6.20 \mathrm{E}-02$ & $3.04 \mathrm{E}-02$ \\
\hline TY-103 & $0.00 \mathrm{E}+00$ & $2.06 \mathrm{E}+02$ & $1.15 \mathrm{E}+05$ & $9.66 \mathrm{E}+03$ & $0.00 \mathrm{E}+00$ & $2.14 \mathrm{E}-03$ & $5.00 \mathrm{E}+00$ & $1.01 \mathrm{E}-01$ & $50 \mathrm{E}+00$ & $76 \mathrm{E}-02$ & $10 \mathrm{E}-02$ \\
\hline TY-104 & $0.00 \mathrm{E}+00$ & $0.00 \mathrm{E}+00$ & $3.33 \mathrm{E}+04$ & $1.54 \mathrm{E}+03$ & $0.00 \mathrm{E}+00$ & $0.00 \mathrm{E}+00$ & $1.45 \mathrm{E}+00$ & $1.60 \mathrm{E}-02$ & 7.24E-01 & 02 & $1.66 \mathrm{E}-02$ \\
\hline TY-105 & $0.00 \mathrm{E}+00$ & $0.00 \mathrm{E}+00$ & $1.54 \mathrm{E}+05$ & $8.83 \mathrm{E}+03$ & $0.00 \mathrm{E}+00$ & $0.00 \mathrm{E}+00$ & $6.70 \mathrm{E}+00$ & $9.19 \mathrm{E}-02$ & $3.35 \mathrm{E}+00$ & $6.89 \mathrm{E}-02$ & $2.06 \mathrm{E}-02$ \\
\hline TY-106 & $0.00 \mathrm{E}+00$ & $0.00 \mathrm{E}+00$ & $9.21 \mathrm{E}+03$ & $1.51 \mathrm{E}+03$ & $0.00 \mathrm{E}+00$ & $0.00 \mathrm{E}+00$ & 4.01E-01 & $1.57 \mathrm{E}-02$ & $2.00 \mathrm{E}-01$ & $1.18 \mathrm{E}-02$ & $5.89 \mathrm{E}-02$ \\
\hline U-101 & $0.00 \mathrm{E}+00$ & $0.00 \mathrm{E}+00$ & $1.55 \mathrm{E}+04$ & $3.16 \mathrm{E}+02$ & $0.00 \mathrm{E}+00$ & $0.00 \mathrm{E}+00$ & $6.74 \mathrm{E}-01$ & $3.29 \mathrm{E}-03$ & $3.37 \mathrm{E}-01$ & $2.47 \mathrm{E}-03$ & $7.32 \mathrm{E}-03$ \\
\hline U-102 & $5.25 \mathrm{E}+04$ & $2.13 \mathrm{E}+03$ & $3.04 \mathrm{E}+05$ & $2.16 \mathrm{E}+04$ & $2.28 \mathrm{E}+00$ & 2.22E-02 & $1.32 \mathrm{E}+01$ & $2.25 \mathrm{E}-01$ & $90 \mathrm{E}+00$ & 1 & $5 \mathrm{E}-02$ \\
\hline U-103 & $0 \mathrm{E}+00$ & $0.00 \mathrm{E}+00$ & $4.11 \mathrm{E}+05$ & $2.57 \mathrm{E}+04$ & $0.00 \mathrm{E}+00$ & $0.00 \mathrm{E}+00$ & $1.79 \mathrm{E}+01$ & $2.68 \mathrm{E}-01$ & $94 \mathrm{E}+00$ & $2.01 \mathrm{E}-01$ & $2.24 \mathrm{E}-02$ \\
\hline U-104 & $0.00 \mathrm{E}+00$ & $0.00 \mathrm{E}+00$ & $8.46 \mathrm{E}+04$ & $7.40 \mathrm{E}+03$ & $0.00 \mathrm{E}+00$ & $0.00 \mathrm{E}+00$ & $3.68 \mathrm{E}+00$ & 7.70E-02 & $1.84 \mathrm{E}+00$ & $5.78 \mathrm{E}-02$ & $3.14 \mathrm{E}-02$ \\
\hline U-105 & $3.52 \mathrm{E}+04$ & $1.24 \mathrm{E}+03$ & $3.70 \mathrm{E}+05$ & $3.34 \mathrm{E}+04$ & $1.53 \mathrm{E}+00$ & $1.29 \mathrm{E}-02$ & $1.61 \mathrm{E}+01$ & $3.48 \mathrm{E}-01$ & $9.58 \mathrm{E}+00$ & $2.74 \mathrm{E}-01$ & $2.86 \mathrm{E}-02$ \\
\hline U-106 & $4.73 \mathrm{E}+04$ & $1.83 \mathrm{E}+03$ & $2.11 \mathrm{E}+05$ & $1.13 \mathrm{E}+04$ & $2.06 \mathrm{E}+00$ & $1.91 \mathrm{E}-02$ & $9.18 \mathrm{E}+00$ & $1.18 \mathrm{E}-01$ & $6.65 \mathrm{E}+00$ & $1.07 \mathrm{E}-01$ & $1.61 \mathrm{E}-02$ \\
\hline U-107 & $1.72 \mathrm{E}+05$ & $5.17 \mathrm{E}+03$ & $2.48 \mathrm{E}+05$ & $4.29 \mathrm{E}+03$ & $7.48 \mathrm{E}+00$ & $5.38 \mathrm{E}-02$ & $1.08 \mathrm{E}+01$ & $4.47 \mathrm{E}-02$ & $1.29 \mathrm{E}+01$ & 8.73E-02 & $6.78 \mathrm{E}-03$ \\
\hline U-108 & $1.31 \mathrm{E}+05$ & $2.28 \mathrm{E}+03$ & $5.96 \mathrm{E}+05$ & $4.23 E+04$ & $5.70 \mathrm{E}+00$ & $2.37 \mathrm{E}-02$ & $2.59 \mathrm{E}+01$ & 4.40E-01 & $1.87 \mathrm{E}+01$ & 1 & $1.90 \mathrm{E}-02$ \\
\hline U-109 & $6.01 \mathrm{E}+04$ & $1.36 \mathrm{E}+03$ & $3.38 \mathrm{E}+05$ & $2.29 \mathrm{E}+04$ & $2.61 \mathrm{E}+00$ & $1.42 \mathrm{E}-02$ & $1.47 \mathrm{E}+01$ & $2.38 \mathrm{E}-01$ & $9.97 \mathrm{E}+00$ & 1.93E-01 & $1.94 \mathrm{E}-02$ \\
\hline U-110 & $0.00 \mathrm{E}+00$ & $0.00 \mathrm{E}+00$ & $1.14 \mathrm{E}+05$ & $2.61 \mathrm{E}+03$ & $0.00 \mathrm{E}+00$ & $0.00 \mathrm{E}+00$ & $4.96 \mathrm{E}+00$ & $2.72 \mathrm{E}-02$ & $2.48 \mathrm{E}+00$ & 2.04E-02 & $8.22 \mathrm{E}-03$ \\
\hline U-111 & $2.54 \mathrm{E}+05$ & $2.26 \mathrm{E}+04$ & $1.25 \mathrm{E}+05$ & $0.00 \mathrm{E}+00$ & $1.10 \mathrm{E}+01$ & $2.35 \mathrm{E}-01$ & $5.44 \mathrm{E}+00$ & $0.00 \mathrm{E}+00$ & $1.38 \mathrm{E}+01$ & $2.35 \mathrm{E}-01$ & $1.71 \mathrm{E}-02$ \\
\hline U-112 & $0.00 \mathrm{E}+00$ & $0.00 \mathrm{E}+00$ & $2.23 \mathrm{E}+04$ & $1.08 \mathrm{E}+03$ & $0.00 \mathrm{E}+00$ & $0.00 \mathrm{E}+00$ & $9.70 \mathrm{E}-01$ & $1.12 \mathrm{E}-02$ & $4.85 \mathrm{E}-01$ & $8.43 \mathrm{E}-03$ & $1.74 \mathrm{E}-02$ \\
\hline U-201 & $0.00 \mathrm{E}+00$ & $0.00 \mathrm{E}+00$ & $1.07 \mathrm{E}+03$ & $1.64 \mathrm{E}+01$ & $0.00 \mathrm{E}+00$ & $0.00 \mathrm{E}+00$ & $4.65 \mathrm{E}-02$ & $1.71 \mathrm{E}-04$ & 2.33E-02 & $1.28 \mathrm{E}-04$ & $5.50 \mathrm{E}-03$ \\
\hline U-202 & $0.00 \mathrm{E}+00$ & $0.00 \mathrm{E}+00$ & $1.07 \mathrm{E}+03$ & $1.64 \mathrm{E}+01$ & $0.00 \mathrm{E}+00$ & $0.00 \mathrm{E}+00$ & $4.65 \mathrm{E}-02$ & $1.71 \mathrm{E}-04$ & 2.33E-02 & $1.28 \mathrm{E}-04$ & $5.50 \mathrm{E}-03$ \\
\hline U-203 & $0.00 \mathrm{E}+00$ & $0.00 \mathrm{E}+00$ & $5.35 \mathrm{E}+02$ & $1.67 \mathrm{E}+01$ & $0.00 \mathrm{E}+00$ & $0.00 \mathrm{E}+00$ & $2.33 \mathrm{E}-02$ & $1.74 \mathrm{E}-04$ & $1.16 \mathrm{E}-02$ & $1.30 \mathrm{E}-04$ & $1.12 \mathrm{E}-02$ \\
\hline U-204 & $0.00 \mathrm{E}+00$ & $0.00 \mathrm{E}+00$ & $1.80 \mathrm{E}+02$ & $8.24 \mathrm{E}+00$ & $0.00 \mathrm{E}+00$ & $0.00 \mathrm{E}+00$ & $7.83 \mathrm{E}-03$ & $8.58 \mathrm{E}-05$ & $3.91 \mathrm{E}-03$ & $6.43 \mathrm{E}-05$ & $1.64 \mathrm{E}-02$ \\
\hline
\end{tabular}

*generated with the assumption that $50 \%$ of sodium in the solid fraction and $75 \%$ of the sulfate would be returned to the liquid feed. 